
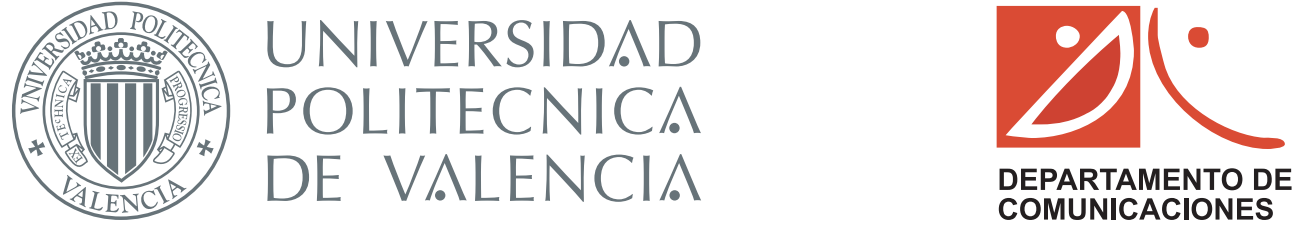

Doctoral thesis submitted for the degree of Doctor of Philosophy in Telecommunications Engineering

\title{
Design and implementation of plasmonic metamaterials and devices
}

Francisco José Rodríguez Fortuño

Valencia, July 2013

Supervisor:

Dr. Alejandro José Martínez Abiétar 

A mis padres. 


\section{Acknowledgments}

This thesis has been, without any doubt, a period of my life full of new experiences, exciting stories, and overall happiness. More important than the academic achievements, I grew personally and emotionally thanks to everyone which I met, talked with or befriended during these years.

During all this time I have enjoyed the guidance of my academic "father" Dr. Alejandro Martínez, I wish to thank you for your close supervision, motivation, and continuous help and support. Also to the very warm guidance provided by Prof. Nader Engheta, Prof. Anatoly Zayats, and Dr. Gregory Wurtz, who not only acted as temporary supervisors but also helped me feel like I was at home in your research groups. I also wish to express my gratitude to Prof. Javier Martí for giving me the opportunity and means to carry on my research in this exciting field of science.

None of this would have been possible without the experience and invaluable help of my scientific "brothers", Carlos García and Rubén Ortuño, who introduced me into the world of research, taught me so much, worked with me in so many projects, and shared with me the excitement of finding so many new ideas, specially on quiet Friday afternoons. Thanks to Mariluz Martínez and Pedro Rodríguez for your fantastic chemistry work in our plasmonic sensors. I am also extremely happy of having worked with Begoña Tomás, María Lorente and Irene Alepuz in your degree and master final projects. Thanks to the great ideas of Pavel Ginzburg at Kings, and to Daniel O'Connor (and Greg) for introducing me to the exciting experimental world. A very special thanks goes to Ashkan Vakil, with whom it was a real pleasure to work side-by-side in our crazy theoretical projects at UPenn, and to Giuseppe Marino for your patience in our experimental measurements at Kings. The great teamwork achieved in the last months with Daniel Puerto and Amadeu Griol at NTC was also incredibly exciting and unforgettable.

The great fun, conversations, and fantastic experiences lived with my friends from NTC in, and specially out of work, will never be forgotten: Carlos García, Rubén Ortuño, Javier García, Jesús Palací, José María Escalante, Guillermo Villanueva, Begoña Tomás, María Lorente, Irene Alepuz, Pere Pérez, Antoine Brimont, Mariluz Martínez, Marta Beltrán, Mercé Llopis, Alexander Bockelt, Claudio Otón, Veronica Toccafondo, Pedro Rodríguez, Daniel Puerto, Jaime García, Ruth Vilar, Javier Herrera, Juan Hurtado, Sara Mas, Ana Gutiérrez, Mariam Aamer, Jose Vicente Galán and many others, thank you.

Many thanks go to my friends from the Engheta Group at UPenn, very specially Uday Chettiar for his friendship and guidance, Victor Torres for the great times, Alexandre Alves, Cristian Della Giovampaola, Fereshteh Abbasi, Ahmed Abdelwahab, Brian Edwards, Humeyra Caglayan, Milin Zhang, Nikolina Jankovic, Matt Zhu, Wenkan Zhu and Mário Silveirinha for being great lab and office friends. Thank you, also, friends in the neighboring UPenn rooms, with whom so many lunch times, dinners, parties, and even trips have been enjoyed: Jero (unforgettable "express" trips), Marco, Songbin, Augusto, Matteo, Nippun, Sid, Cristian, Gianca, Serkan, Chiara, Scott, Sam, Nick, Alberto and Lisha.

A very special thanks goes to my Philadelphia housemates Jose Franch-Ballester and Cristina Enguídanos for their kindness, the great moments, the movies, the games, the tasty food and for making me feel like home. Many thanks to Bryan Cameron in Philly for his helping hand in a difficult moment. 
Also unforgettable is my stay in London, thanks entirely to the amazing friends that I enjoyed at King's College: Andrés Barbosa Neira and Angela, Silvia Peruch, Cillian McPolin, Ryan McCarron, Paulina Segovia, Ali Mansourian, William Wardley, Michelle Gaio, Jean-Sebastien Bouillard, Alexey Krasavin, Nikolaos Vasilantonakis, Julia Kilpatrick, Riccardo Sapienza, and Wayne Dickson, among others from the department. The time enjoyed with Alex Tatay, Cris, and their London friends was great. Thanks go to my housemates in Hampstead, whom if listed would include at least three Sarahs!

A very sincere gratitude goes to Drs. Miguel Beruete, Mário Silveirinha, Nikos Papanikolau, Ricardo Marqués, José Sánchez Dehesa and José A. Sánchez Gil, for their very helpful constructive comments regarding the preparation and defense of the thesis. A special thanks goes to Miguel Navarro for your guidance, the good moments spent in Valencia, and your kind help easing my trip to Philadelphia and specially your warm welcome at London.

A mis amigos de toda la vida. Algunos de vosotros vinisteis a visitarme (o al menos esa fue una buena excusa) al lejano Nueva York: David, Marcos, Monty, Hernán, Pablo, Salva, Tomás, y ambos Borjas. Increíble. Otros vinisteis a Londres: Alber, Iván, Rober, Nuria, Jero, Borja y Fer. Mil gracias. Y a los demás: Alex Mira, Oliver, Moreno, Pablo, Fer, Paula, Sandra, Esther, Raquel, DVD, Basto, Laura(s), y muchos otros. ¡Qué bien se lo pasa uno con todos vosotros! Gracias a todos por acordaros tanto de mí. Debo muchísimo a Roberto por tu inmensa amistad y las grandes experiencias vividas como compañeros de piso durante unos meses verdaderamente emocionantes, divertidos, e inolvidables. Gracias especiales a Alberto por tu gran y eterna amistad, y por venir a verme a mi defensa de tesis. También agradecer a los amigos de teleco por la inspiración y el ejemplo contínuo que dáis del trabajo duro y la superación, pero también por las divertidísimas quedadas, proyectos, partidas al ordenador, y la fuerte amistad que siempre habéis demostrado: Arturo, Borja, Samu, Luis, Ricardo, y Eloi. Fuertes ánimos quiero enviar también para todos los miembros de Red Little House y su inspirador entusiasmo. Un agradecimiento por las divertidas quedadas junto al grupo de amigas de teleco de María e Irene. Me dejo a muchos otros amigos que me han hecho disfrutar estos años, sois demasiados como para haceros justicia en tan poco espacio, pero sabéis quienes sois: abrazos y besos para todos vosotros.

Por último, agradezco enormemente a toda mi familia, incluyendo tíos, primos y sobrinos. Doy gracias a mis hermanas Julia y Paula, que tanto me quieren, me han cuidado siempre, y me han enseñado a ser como soy. Y también a las nuevas extensiones de familia: Mario, Elisa, Paulina, David, Celia, Gilda y Eduardo. Infinitas gracias a Paula, Mario y “Lulo" por acogerme en casa durante esos geniales meses. A mis padres, más que a nadie, por hacer que todo esto sea posible, en todos los sentidos de la palabra, queriéndome, apoyándome y animándome siempre en todo lo que he hecho. Me quedará un recuerdo muy fuerte de mi pequeño "Kiket", que estuvo siempre a mi lado. Y Tania, muchas gracias por enseñarme y compartir tantas cosas conmigo: increíbles viajes, nuevas emociones, inolvidables historias, grandes experiencias, innumerables risas y felicidad. 


\footnotetext{
${ }^{0}$ Personal financial support is gratefully acknowledged to the master studies' grant from La Caixa and to the predoctoral FPI grant of Generalitat Valenciana, which gave me the opportunity and means of carrying out this research and performing such enriching stays abroad.
} 


\section{Contents}

Introduction and objectives $\quad 1$

1 Fundamentals of plasmonics 5

1.1 Introduction to plasmonics . . . . . . . . . . . . 5

1.2 Analytical description of surface plasmons . . . . . . . . . . 8

1.2.1 A classical phenomenon . . . . . . . . . . . . . . 8

1.2.2 Optical properties of metals . . . . . . . . . . . . 9

1.2.3 General method for the calculation of surface plasmon modes . 10

1.3 Surface plasmon in a smooth metal interface . . . . . . . . . . . . . 14

1.4 Excitation of surface plasmons . . . . . . . . . . . . 17

2 Negative index metamaterials 19

2.1 Metamaterials - what are they? . . . . . . . . . . . . . . . . . . 19

2.2 Negative index media . . . . . . . . . . . . . . . . . 22

2.3 The perfect lens . . . . . . . . . . . . . . . . . . . 24

2.4 State of the art . . . . . . . . . . . . . . . 26

2.5 Our work (A,B): A u-shaped nanowire meta atom NIM . . . . . . . . . 29

2.6 Backward plasmonic modes and plasmon lenses . . . . . . . . . . . 30

2.7 Our work (C): NIM based on coaxial plasmonic waveguides . . . . . . 33

2.8 PAPER A . . . . . . . . . . . . . . . . . 35

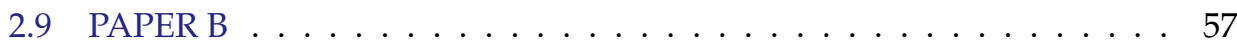

2.10 PAPER C . . . . . . . . . . . . . . . . . 71

3 Plasmonic analogues of quantum phenomena 79

3.1 Fano resonances . . . . . . . . . . . . . . . . . 80

3.2 Electromagnetically Induced Transparency . . . . . . . . . . . . . . . . . . 81

3.3 Our work (D): SRRs inside a photonic crystal . . . . . . . . . . . . . 85

3.4 Our work (E): Plasmonic analogue of Hanle effect . . . . . . . . . . . . 87

3.5 Our work: Electric analogue of Meissner levitation . . . . . . . . . . . . 89

3.6 PAPER D . . . . . . . . . . . . . . . . . . . 91

3.7 PAPERE . . . . . . . . . . . . . . . . . 101 
4 Plasmonic sensing $\quad 115$

4.1 Fundamentals of plasmonic sensing . . . . . . . . . . . . . 115

4.2 Types of plasmonic sensors and comparison . . . . . . . . . . 116

4.3 State of the art of LSPR sensors . . . . . . . . . . . . . . . . . . . . . . . . . . . . . . .

4.4 Our work (F): Plasmonic nano-cross LSPR sensor . . . . . . . . . . . . . 119

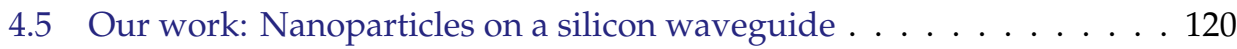

4.6 PAPER F . . . . . . . . . . . . . . . . . 123

5 Plasmonic interference phenomena 131

5.1 Some plasmonic applications of interference . . . . . . . . . . . 131

5.2 Our work (G): Plasmonic loss reduction using multimode interference 133

5.3 Near field interference phenomena . . . . . . . . . . . . . . . 135

5.4 Our work $(\mathrm{H})$ : Near field interference for unidirectional excitation . . . 135

5.5 PAPER G . . . . . . . . . . . . . . . . . . . . 141

5.6 PAPER H . . . . . . . . . . . . . . . . . . 153

General discussion of results and conclusions 163

$\begin{array}{ll}\text { References } & 167\end{array}$

$\begin{array}{lr}\text { Author's merits } & 189\end{array}$

$\begin{array}{ll}\text { Abstract } & 195\end{array}$ 


\section{Introduction and objectives}

This thesis describes the work that we performed under the doctorate program of the Universitat Politècnica de València (UPV), for the degree of Doctor of Philosophy (PhD) in Telecommunications Engineering.

The research presented here was carried out at the Nanophotonics Technology Center of the UPV, under the supervision and guidance of Dr. Alejandro Martínez, and during the two stays performed by this doctoral student, at the Engheta Group in the University of Pennsylvania ${ }^{1}$ under the guidance of Prof. Nader Engheta, and at the Experimental Biophysics \& Nanotechnology group in King's College London ${ }^{2}$ under the guidance of Prof. Anatoly V. Zayats.

The different results derived from our research are presented as a collection of papers published in international peer-reviewed scientific journals. The papers are grouped into different chapters according to their field of study, so each chapter presents an introduction to the topic, describes the current state of the art, and gives an overview of our work. The more specific details and results of each of our contributions is left to the papers themselves, which are included reprinted at the end of each chapter.

Among other research projects, the results from this thesis form part of the Engineering Metamaterials project in the CONSOLIDER program, funded by the Spanish government for high-level scientific institutions working at the edge of human knowledge, directed at both basic and applied science. Although our results might seem to cover a wide variety of topics, they all revolve around the common field of plasmonics.

\section{Field of study: Plasmonics}

Plasmonics studies the existence and behavior of self-sustained waves of light coupled to free electrons in metals. Although the field is grounded on classical physics and was developed during the $20^{\text {th }}$ century, in recent years - with the advent of nanoscience and nanotechnology - plasmonics has come to the forefront of current

\footnotetext{
${ }^{1}$ From March to November 2011.

${ }^{2}$ From May to November 2012.
} 
nanotechnology research. Plasmonics is one of the 23 milestones in the history of photonics according to the scientific journal Nature [1]. A "surface plasmon resurrection" is currently witnessed, in words of the Nature Photonics editorial staff [2], as the number of publications in the field grows. Among the many fascinating applications of plasmonics, those of information processing [3], sensing [4], imaging [5], and light manipulation [6] stand out.

This thesis is also grounded on the closely related field of metamaterials [7], artificial structures that achieve the control of light propagation in their interior through the structure of the constituent so-called meta atoms, enabling properties not possible in conventional materials, and allowing —in principle — truly extraordinary applications such as the perfect lens [8] or the cloak of invisibility [9, 10]. Like plasmonics, the field of metamaterials was also named one of the 23 milestones in the history of photonics [11]. Both fields are closely intertwined, because very often the meta atoms are designed with metallic structures working at optical frequencies, thus involving plasmonics in their design and understanding [12].

\section{Objectives}

The world's research activity in plasmonics and metamaterials is currently surging, and novel applications are being born constantly. This thesis is an attempt at contributing to the field with our own advances.

Our goal is to devise, design and, whenever possible, experimentally realize and measure, new plasmonic devices and applications, including novel metamaterials. The requirements to achieve this aim are a solid theoretical foundation of the fields that enables the development of a physical intuition, an adequate use of the appropriate electromagnetic simulation software - be it commercial, open source, or self-codedand the appropriate experience and facilities for the nanofabrication and measurement of nanotechnological plasmonic devices.

\section{Structure of the thesis}

This book consists of the following sections:

- It begins with the present Introduction and objectives, and then proceeds with the main materials of the thesis presented in the following numbered chapters.

- Chapter 1: Fundamentals of plasmonics, describes the fundamental principles and physical origins of plasmonics. It includes the analytical derivation of the existence of surface plasmons from Maxwell's equations of electromagnetism and the metal properties. Although no papers are attached to this chapter, it will serve as the foundation for the rest of the chapters and results presented in this thesis. 
After these introductory sections, each of the following chapters deal with the different sub-fields of our works, with our journal contributions attached.

- Chapter 2: Negative index metamaterials, gives a brief history, state of the art, and applications of negative index plasmonic metamaterials. It includes our works [PAPER A, PAPER B, PAPER C].

- Chapter 3: Plasmonic analogues of quantum phenomena, describes some novel plasmonic applications that have arisen from physical analogies to known quantum phenomena. After the existing analogues and applications are described, we present our works [PAPER D, PAPER E]. The still unpublished [Paper 19] is also outlined.

- Chapter 4: Plasmonic sensing, introduces the applications of plasmonic-based chemical and biological sensors, reviewing the field and its expected evolution, and presents our experimental contribution [PAPER F].

- Chapter 5: Plasmonic interference phenomena, introduces the importance of interference in plasmonics and other fields of physics, and after describing some selected applications of plasmonic interference, our works [PAPER G, PAPER H] are presented.

- To close this sequence of chapters, there is a General discussion of results and conclusions, where the main results are summarized and unified. We conclude the thesis with a discussion of how the results are related to one another, and we justify how we accomplished our initial objectives.

At the end of the book the following bibliographic sections can be found:

- The section References lists all the works that are cited throughout all chapters, numbered sequentially in order of appearance. In the electronic version of this thesis, each reference includes a link to the appropriate online journal paper or resource through the use of the digital object identifier (doi).

- After the references, the complete list of Author's merits are given at the end of the book in page 189, including the main contributions to international journals, that are attached at the end of the appropriate chapters [PAPER A]-[PAPER H], the additional contributions to international journals as a co-author [Paper 9][Paper 18] and the still unpublished main contributions, available in pre-print servers [Paper 19, Paper 20]. The conference proceedings resulting from oral and poster presentations are also given in [Conf 1]-[Conf 25]. 


\section{Chapter 1}

\section{Fundamentals of plasmonics}

But what is plasmonics? "You just have Maxwell's equations, some material properties and some boundary conditions, all classical stuff - what's new about that?" Well, would you have predicted that just by imposing appropriate structure on a metal one could make a synthetic material that would turn Snell's law on its head? Or that you could squeeze light into places less that one hundredth of a wavelength in size?

-Prof. Bill Barnes, Foreword to

S. A. Maier, Plasmonics: Fundamentals and Applications [13].

THE RESEARCH WORK presented in this thesis covers a wide variety of topics, yet it ultimately all revolves around plasmonics, a fascinating field of science that studies the behavior of light at metal surfaces, and into which interest is lately surging due to its enormous potential. In this first chapter, an introduction to plasmonics is given, including a discussion of its physical origin, a brief historical perspective, and a discussion of the applications and challenges of present plasmonic technology. The chapter details a method for the analytic description of plasmonic modes, and studies in detail the simplest plasmonic scenario - the surface plasmon that exists at the interface of a smooth metal and a dielectric,- discussing its properties, its excitation, and forming the foundation for many of the following topics in this thesis.

\subsection{Introduction to plasmonics}

Plasmonics forms part of the broad field of nanophotonics, a field of science and engineering which explores the confinement and control of high frequency electromagnetic fields - typically infrared or visible light- over sizes on the order of the 
wavelength or smaller, i.e. on the nanometer scale ${ }^{1}$. Nanophotonics is an emergent field of study motivated by the rapid advance of nanotechnology [14]. In turn, the field of plasmonics is the part of nanophotonics that deals with the interaction between electromagnetic radiation and conduction electrons in the surface of metals, leading to an enhanced optical near-field confined over sub-wavelength dimensions [13]. A very basic physical explanation of plasmonics can be given as follows:

- Light, as any other electromagnetic wave, causes forces in charges. The conduction electrons in metals show little resistance to this force, and at low frequencies, their movement follows easily the driving electromagnetic field. This movement of charges in turn creates a scattered field, which exactly cancels the electromagnetic field inside the metal, and constitutes the reflected fields outside. Metals at low frequencies can therefore be modeled using the perfect or good conductor approximation.

- At optical frequencies, the movement of the electrons lags behind that of the rapidly varying electromagnetic field. This allows some penetration of the field into the metal, and if the adequate relation of material properties is satisfied, the collective motion of the electrons induces an electromagnetic field near the surface which in turn maintains the collective electron motions, giving rise to a self sustained excitation which may propagate closely confined along the surface of the metal. This excitation is called a surface plasmon polariton (SPP), or simply a surface plasmon.

- In the case of a finite metallic nanoparticle, the small size of the nanoparticle allows the conduction electrons to oscillate collectively, with a restoring force coming from the walls of the finite size particle, leading to plasmonic resonances. These resonances are not propagating but rather localized to the nanoparticle, and are called a localized surface plasmon resonance (LSPR). The resonance wavelength depends on the size, shape, and surrounding environment of the nanoparticle.

Both type of excitations, SPPs and LSPRs, form the basic ingredients of plasmonics.

Knowledge of plasmonic phenomena dates from the early $20^{\text {th }}$ century. Localized surface plasmon resonances on metallic spheres where given a definitive mathematical explanation by Gustav Mie in the year 1908 [15] by solving Maxwell's equations on spherical gold particles, applying wave expansion into spherical waves. He successfully explained the colors of colloidal solutions of metal nanoparticles, which had been present in tainted glass in windows and other artifacts since ancient times. Fig. 1.1 shows the most celebrated example, an ancient Roman cup whose color changes when illuminated from the front or from the back.

Propagating surface plasmons, on the other hand, took some time for a unified theory to be established after the first experimental observations. Theoretical work

\footnotetext{
${ }^{1}$ The wavelength of visible light covers the range $\lambda_{\text {vis }}=380 \mathrm{~nm}-700 \mathrm{~nm}$, and that of infrared light covers roughly the range from $\lambda_{I R}=700 \mathrm{~nm}$ to as far as $1 \mathrm{~mm}$ (far infrared).
} 




Figure 1.1: The Lygurcus cup, probably made in Alexandria or Rome around 300AD, has embedded plasmonic nanoparticles in its glass. The glass appears green in reflected light and red in transmitted light.

on waves propagating along the surface of conductors with finite conductivity was originally studied by Sommerfeld $[16,17]$ in the context of radio waves over Earth's surface. The observation by R. W. Wood of anomalous intensity drops in the spectra of light reflected at metallic gratings in 1902 [18] had to wait almost 40 years before it was given an explanation [19] in connection to Sommerfeld's surface waves. Later, interactions taking place at metallic surfaces were recorded via diffraction of electron beams by Ritchie [20], who later linked this to the original work on diffraction gratings [21]. At the same time, the excitation of Sommerfeld's surface waves at optical frequencies was achieved and demonstrated using prism coupling [22], making the experimentation with surface plasmons easily accessible to many researchers. All this led to a good understanding and a unification of all these phenomena in the form of surface plasmon polaritons.

Today, there is a surge in interest toward surface plasmon nanophotonics [2] coincident with the rise of the fields of nanoscience and nanotechnology. The unprecedented ability of plasmonic structures to concentrate light into deeply subwavelength volumes [23] promises a wide variety of new nanophotonic technology, of which we can enumerate a few:

- The enormous field enhancement achieved in plasmonic resonances presents exciting new applications for high performance sensing [4, 24, 25], spectroscopy [26] and non-linear enhancement [27].

- Current commercial electronic devices are severely limited for the transfer of high volumes of information. This problem has been conventionally overcome for high distance transmission using photonic circuits and optical fiber, but current trends suggest the need for this information transfer to occur within a same electronic chip (e.g. for ultra high bandwidth communication between different processors in a multi core device). In this sense, current nanophotonic technology 
[28] lacks the required integration, due to its micron-sized components whose size is fundamentally limited by the diffraction of light. Plasmonic circuits are therefore excellent candidates for the merging of photonics with electronics [3] thanks to their sub-diffraction confinement capabilities.

- Fascinating novel research is directed toward the high resolution sub-diffraction imaging capabilities of plasmonic lenses [5, 29], allowing ultra dense storage of information and nanolithography [30].

- Antennas are an old and respectable subject in radiofrequency engineering. The exciting possibility to realize plasmonic nanoantennas that work with light is a topic undergoing intense research [31, 32].

- The very exciting field of metallic nanohole arrays exhibiting extraordinary optical transmission allows advanced filtering techniques and light control [33].

- The ultimate light manipulation and control is achieved with metamaterials $[7,12,34]$ which are intimately linked to plasmonics.

All these are only some selected applications and phenomena among many others. It is clear that the field of plasmonics well-deserves an in-depth study. The main challenge in plasmonics is to overcome the Joule losses [35] intrinsic to the movement of electrons in the metal.

\subsection{Analytical description of surface plasmons}

\subsubsection{A classical phenomenon}

Plasmonics is a subject firmly based in classical physics, and in particular in classical electromagnetism. In truth, when particle sizes or separations get very small (about $10 \mathrm{~nm}$ or less), quantum effects can become important. However, for typical sizes including all of the various plasmonic phenomena described in this thesis, we can ignore quantum effects, and plasmonics is entirely governed by the classical macroscopic Maxwell equations, given by:

$$
\begin{aligned}
\nabla \cdot \mathbf{D} & =\rho_{\text {ext }}, \\
\nabla \cdot \mathbf{B} & =0, \\
\nabla \times \mathbf{E} & =-\partial \mathbf{B} / \partial t, \\
\nabla \times \mathbf{H} & =\mathbf{J}_{\text {ext }}+\partial \mathbf{D} / \partial t,
\end{aligned}
$$

where $\mathbf{E}$ is the electric field, $\mathbf{B}$ is the magnetic flux density, $\mathbf{H}$ is the magnetic field, $\mathbf{D}$ is the electric displacement, and $\rho_{\text {ext }}$ and Jext are the external charge density and current, respectively, defined as the set of sources that drive the system. The other sources, i.e. the currents and charges owing to the polarization and magnetization 
of materials, are contained within the auxiliary macroscopic vector magnitudes $\mathbf{D}$ and $\mathbf{H}$, which are defined in relation to the material polarization $\mathbf{P}$ (density of electric dipoles $d \mathbf{p} / d v$ ) and magnetization $\mathbf{M}$ (density of magnetic dipoles $d \mathbf{m} / d v$ ) by:

$$
\begin{aligned}
& \mathbf{D}=\varepsilon_{0} \mathbf{E}+\mathbf{P}, \\
& \mathbf{H}=\frac{1}{\mu_{0}} \mathbf{B}-\mathbf{M},
\end{aligned}
$$

where $\varepsilon_{0}$ and $\mu_{0}$ are the electric permittivity and magnetic permeability of vacuum ${ }^{2}$ and we have neglected the electric quadrupole and higher moments [36]. When the materials considered are linear ${ }^{3}$ and isotropic (e.g. $\mathbf{P}=\varepsilon_{0} \chi \mathbf{E}$ where $\chi$ is the complex susceptibility), eqs. (1.2a) and (1.2b) can be re-written into the following so-called constitutive relations

$$
\begin{aligned}
& \mathbf{D}=\varepsilon_{0} \varepsilon \mathbf{E}, \\
& \mathbf{B}=\mu_{0} \mu \mathbf{H},
\end{aligned}
$$

where $\varepsilon=\varepsilon^{\prime}+i \varepsilon^{\prime \prime}$ is the relative electric permittivity and $\mu=\mu^{\prime}+i \mu^{\prime \prime}$ is the relative magnetic permeability. To discover the presence of surface plasmons arising from these equations, we just need to substitute the material properties of the metal. In the optical regime, materials are non-magnetic $\mu \approx 1$, so metals can be entirely characterized by their complex $\varepsilon$.

\subsubsection{Optical properties of metals}

At low frequencies, the metals are modeled as perfect ${ }^{4}$ or good conductors. At optical frequencies, however, a phase lag starts to appear between the driving field and the movement of the electrons. The metal is then best modeled by a complex dispersive permittivity $\varepsilon(\omega)=\varepsilon^{\prime}(\omega)+i \varepsilon^{\prime \prime}(\omega)$. The two models are in fact equivalent, differing only in their interpretation of the currents in metals ${ }^{5}$.

A very good model for a metal is that of a free electron plasma on a fixed background of positive charges, subject to an external electric field $\mathbf{E}$. It is straightforward to obtain the dielectric function $\varepsilon(\omega)$ for such a free electron gas. Newton's equation of motion

\footnotetext{
${ }^{2} \varepsilon_{0}=1 /\left(\mu_{0} / c_{0}^{2}\right) \approx 8.854 \times 10^{-12} \mathrm{~F} / \mathrm{m}, \quad \mu_{0}=4 \pi \times 10^{-7} \approx 1.257 \times 10^{-6} \mathrm{H} / \mathrm{m}, \quad c_{0}=299792458 \mathrm{~m} / \mathrm{s}$

${ }^{3}$ If materials are linear, even for non-monochromatic waves and material dispersion, we can work with the Fourier transform of the fields $\mathbf{E}(\omega, \mathbf{k})=\iint \mathcal{E}(t, \mathbf{r}) e^{i \omega t} e^{-i \mathbf{k} \cdot \mathbf{r}} d t d \mathbf{r}$ so that $\varepsilon=\varepsilon(\omega, \mathbf{k})$ and $\mu=\mu(\omega, \mathbf{k})$ can show spatial and temporal dispersion. In that case, eqs. (1.3a) and (1.3b) are still valid in Fourier space, but in space and time notation they represent a convolution. The spatial dispersion can be neglected $[\varepsilon(\omega, \mathbf{k})=\varepsilon(\omega)]$ when the wavelength in the material is greater than all characteristic dimensions. Otherwise, nonlocal effects should be taken into account $[37,38]$. If we neglect spatial dispersion, we can work with phasor notation of time-varying fields $\mathcal{E}(t, \mathbf{r})=\mathfrak{R}\left[\mathbf{E}(\mathbf{r}) e^{-i \omega t}\right]$, as is done throughout this thesis.

${ }^{4}$ When the conductivity $\sigma$ is very high $(\sigma \rightarrow \infty), \mathbf{E}=0$ inside the perfect conductor.

${ }^{5}$ For the conductor model, a current $\mathbf{J}_{\sigma}=\sigma \mathbf{E}$ is developed inside the metal. For the dielectric model, the current is considered to come from the polarization $\mathbf{J}_{\varepsilon}=-i \omega \mathbf{P}$. By equating both $\mathbf{J}_{\sigma}=\mathbf{J}_{\varepsilon}$, together with eqs. (1.2a) and (1.3a) we arrive at the fundamental relationship $\varepsilon=1+i \sigma / \varepsilon_{0} \omega$.
} 
for the electrons is $m \ddot{\mathbf{x}}=-e \mathbf{E}$ where $m$ is the mass of an electron, $-e$ is its charge, and $\mathbf{x}$ is the displacement of the electron with respect to a rest position - the notation $\ddot{\mathbf{x}}$ represents the second time derivative, i.e. acceleration.- We can further refine the model by adding the damping of their motion due to collisions, with a characteristic collision frequency $\gamma=1 / \tau$ where $\tau$ is the relaxation time of the electron gas. The equation of motion is then

$$
m \ddot{\mathbf{x}}=-e \mathbf{E}-m \gamma \dot{\mathbf{x}} .
$$

Assuming a harmonic time dependence on the driving field, we can substitute $\dot{\mathbf{x}} \rightarrow-i \omega \mathbf{x}$ and $\ddot{\mathbf{x}} \rightarrow-\omega^{2} \mathbf{x}$, and thus solve eq. (1.4) as

$$
\mathbf{x}=\frac{e}{m\left(\omega^{2}+i \gamma \omega\right)} \mathbf{E},
$$

giving a linear relation between the movement of electrons and the incident field. This allows a linear dielectric model to be used. Each electron will produce a dipole moment $\mathbf{p}=-e \mathbf{x}$. If there are $n$ electrons per unit volume, the total polarization $\mathbf{P}$ of the material will be $\mathbf{P}=n \mathbf{p}=-n e \mathbf{x}$. Substituting this into eqs. (1.2a) and (1.3a) we obtain the free electron dielectric function:

$$
\varepsilon(\omega)=1-\frac{\omega_{p}^{2}}{\omega^{2}+i \gamma \omega} \quad \text { with } \quad \omega_{p}=\frac{n e^{2}}{\varepsilon_{0} m} .
$$

Equation (1.6) is commonly referred to as the Drude model, and it is a very good approximation to the material properties of noble metals at optical frequencies. To demonstrate the validity of the Drude model, Fig. 1.2 shows the experimentally measured values of gold and silver, compared to the Drude model commonly used in the literature and throughout this thesis. For a better fit to experimental data, the constant term 1 in eq. (1.6) is commonly substituted with a parameter $\varepsilon_{\infty}$, corresponding to the limiting value of $\varepsilon(\omega \rightarrow \infty)$. We can see that $\varepsilon(\omega)$ is negative below a certain frequency, a main characteristic of metals allowing the existence of surface plasmons.

\subsubsection{General method for the calculation of surface plasmon modes}

Once we know the material parameters, we are ready to solve the propagating modes in plasmonic waveguides. To do this, we follow a general method commonly used in dielectric waveguides: we write down the solutions of Maxwell's equations on the region of each different material, in terms of unknown coefficients, and we solve the values of the coefficients by enforcing the appropriate boundary conditions. The details of the method can be found in many electromagnetic engineering textbooks, but we will follow the very general method described in ref. [42] which makes use of the vector potentials $\mathbf{A}$ and $\mathbf{F}$. The vector potentials are defined such that:

$$
\begin{aligned}
\nabla^{2} \mathbf{A}+k^{2} \mathbf{A} & =-\mu_{0} \mu \mathbf{J}, \\
\nabla^{2} \mathbf{F}+k^{2} \mathbf{F} & =-\varepsilon_{0} \varepsilon \mathbf{J}_{\mathbf{M}},
\end{aligned}
$$



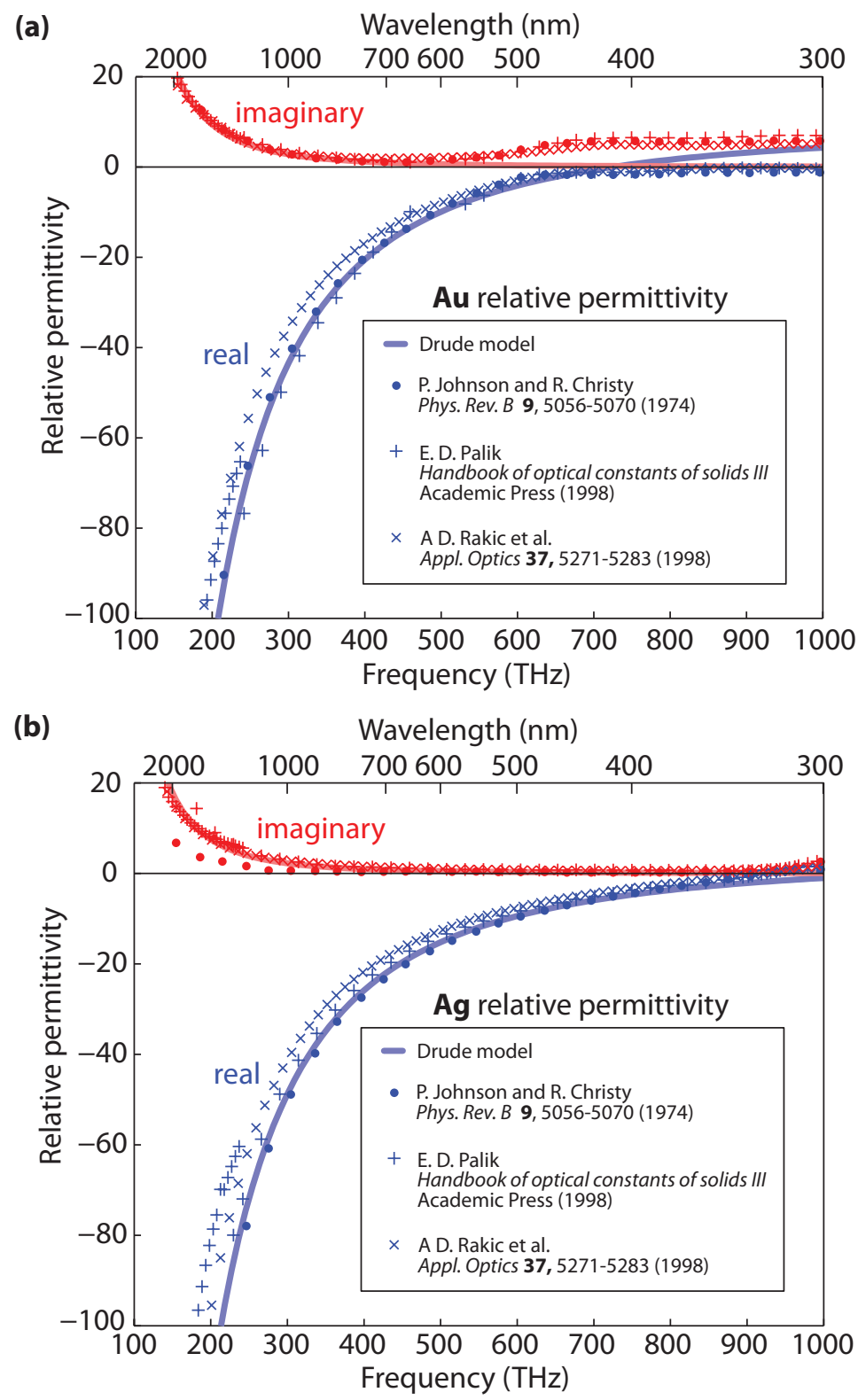

Figure 1.2: Measured relative permittivity values of (a) gold and (b) silver, taken from Refs. [39-41], compared with the Drude model fit to Johnson and Christy data [41] typically used in the metamaterials literature and throughout this thesis: Au: $\left(\varepsilon_{\infty}=9, \omega_{p}=1.3673 \times 10^{16} \mathrm{rad} / \mathrm{s}, \gamma=1.0027 \times 10^{14} \mathrm{~s}^{-1}\right), \mathbf{A g}:\left(\varepsilon_{\infty}=3.7, \omega_{p}=\right.$ $\left.1.3673 \times 10^{16} \mathrm{rad} / \mathrm{s}, \gamma=8 \times 10^{13} \mathrm{~s}^{-1}\right)$. 
where $k^{2}=\omega^{2} \mu_{0} \varepsilon_{0} \mu \varepsilon$ is the wavenumber of a medium, $\mathbf{J}$ is the electric current density and $\mathbf{J}_{M}$ is the magnetic current density ${ }^{6}$. The electric and magnetic fields can be obtained from the potentials by using:

$$
\begin{aligned}
& \mathbf{E}=i \omega \mathbf{A}+i \frac{c_{o}^{2}}{\omega \mu \varepsilon} \nabla(\nabla \cdot \mathbf{A})-\frac{1}{\varepsilon_{0} \varepsilon} \nabla \times \mathbf{F}, \\
& \mathbf{H}=i \omega \mathbf{F}+i \frac{c_{o}^{2}}{\omega \mu \varepsilon} \nabla(\nabla \cdot \mathbf{F})+\frac{1}{\mu_{0} \mu} \nabla \times \mathbf{A},
\end{aligned}
$$

where $c_{o}=1 /\left(\varepsilon_{0} \mu_{0}\right)^{1 / 2}$ is the speed of light. Equations (1.7a), (1.7b) and (1.8) are an alternative and equivalent representation of Maxwell's equations.

The method is specially well suited for solving waveguide modes. If we classify the modes of a waveguide into $\mathrm{TE}_{z}$ and $\mathrm{TM}_{z}$ modes ${ }^{7}$, it can be shown [42] that their solutions obey the following simplifications:

$$
\begin{aligned}
& \left\{\begin{array}{c}
\mathbf{A}=A_{z} \hat{\mathbf{z}} \\
\mathbf{F}=0
\end{array}\right\} \text { for } \mathrm{TM}_{z} \text { modes, } \\
& \left\{\begin{array}{c}
\mathbf{A}=0 \\
\mathbf{F}=F_{z} \hat{\mathbf{z}}
\end{array}\right\} \text { for } \mathrm{TE}_{z} \text { modes. }
\end{aligned}
$$

This turns eqs. (1.7a) and (1.7b) into a scalar wave equation. It can be shown ${ }^{8}$ that plasmonic modes are always $\mathrm{TM}_{z}$, so solving the geometry for the vector potential scalar component $A_{z}$ is sufficient. For waveguided modes, there are no sources $\left(\mathbf{J}=\mathbf{J}_{\mathbf{M}}=0\right)$, and the solutions to the homogeneous scalar wave equation are very well known in various coordinate systems, namely:

- Rectangular coordinates: A general solution to $A_{z}$ for the $i$-th medium is ${ }^{9}$ :

$$
A_{z i}(x, y, z)=\left[A_{i} e^{\gamma_{y i} y}+B_{i} e^{-\gamma_{y i} y}\right] e^{\gamma_{z} z} \text { with } \gamma_{y i}^{2}+\gamma_{z}^{2}=-k_{i}^{2},
$$

where $A_{i}, B_{i}$ are unknown coefficients, $k_{i}^{2}=k_{0}^{2} \mu_{i} \varepsilon_{i}=\omega^{2} \mu_{0} \varepsilon_{0} \mu_{i} \varepsilon_{i}$ is the wavenumber of each medium, and $\gamma_{(x / y / z) i}$ are the complex propagation constants in each direction ${ }^{10}$. This solution is used for the calculation of modes in waveguides composed of $\mathrm{N}$ planar slabs as shown in Fig. 1.3(a).

\footnotetext{
${ }^{6}$ In our original display of the Maxwell equations we did not include the possibility of magnetic currents. Although magnetic charges are generally believed to be not physically realizable, effective magnetic currents are an artificial construct useful for engineers in some practical cases. For all our purposes, however, we will take $\mathbf{J}_{\mathbf{M}}=0$.

${ }^{7} \mathrm{TE}_{z}$ modes imply that $\mathbf{E}$ is perpendicular to $\mathrm{z}$, while $\mathrm{TM}_{z}$ modes imply that $\mathbf{H}$ is perpendicular to $\mathrm{z}$.

${ }^{8} \mathrm{In} \mathrm{TE}_{z}$ modes, there is no electric field perpendicular to the metal interface. This means that there can't be surface charge accumulation, which is a necessary condition for plasmonic modes.

${ }^{9} \mathrm{~A}$ more general solution to the problem would actually be written as $A_{z i}(x, y, z)=\left[A_{i} e^{\gamma_{y i} y}+B_{i} e^{-\gamma_{y i} y}\right]$ $\left[C_{i} e^{\gamma_{x i} x}+D_{i} e^{-\gamma_{x i} x}\right]\left[E_{i} e^{\gamma_{z i} z}+F_{i} e^{-\gamma_{z i} z}\right]$, but we made the assumption that propagation occurs only along $+z$ and there is invariance along $x$ i.e. $\gamma_{x i}=0$ (this is equivalent to reorienting the $\mathrm{z}$ axis). Also, continuity of the fields requires all of the $\gamma_{z i}=\gamma_{z}$ to be equal.

${ }^{10}$ The real part represents evanescent attenuation and the imaginary part represents propagation, e.g. $\gamma_{z}=-\alpha_{z}(\mathrm{~Np} / \mathrm{m})+i k_{z}(\mathrm{rad} / \mathrm{m})$.
} 

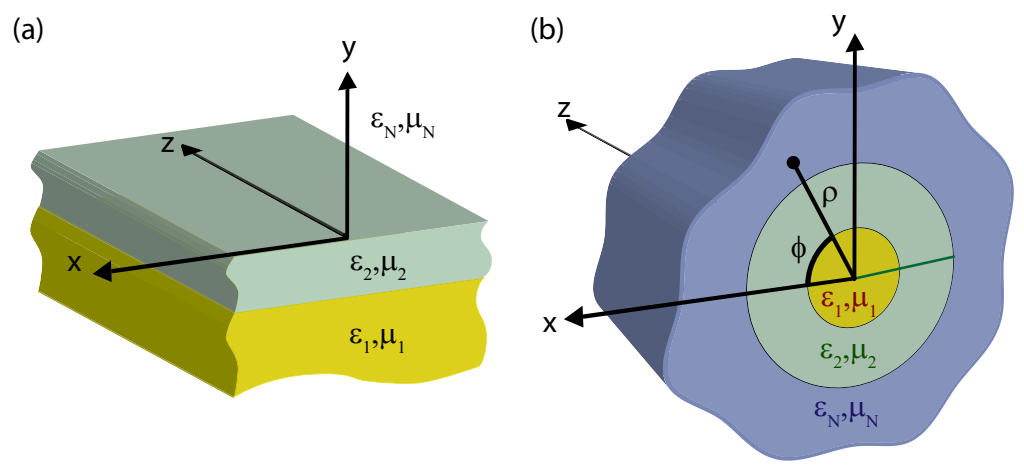

Figure 1.3: Geometry of (a) planar and (b) cylindrical waveguides.

- Cylindrical coordinates: A general solution to $A_{z}$ for the $i$-th medium is ${ }^{11}$ :

$$
A_{z i}(\rho, \phi, z)=\left[A_{i} I_{m}\left(\gamma_{\rho i} \rho\right)+B_{i} K_{m}\left(\gamma_{\rho i} \rho\right)\right] \cos (m \phi) e^{\gamma_{z} z} \quad \text { with } \quad \gamma_{\rho i}^{2}+\gamma_{z i}^{2}=-k_{i}^{2}
$$

where $I_{m}$ and $K_{m}$ are the modified Bessel functions of the first and second kind ${ }^{12}$ and the integer $m=\{0,1, \ldots\}$ can be chosen at will to obtain different families of modes, with different angular variation. This solution is used for waveguides composed of coaxial cylindrical layers as shown in Fig. 1.3(b).

Solutions to the wave equation in other coordinate systems are known and can be found in electromagnetic textbooks [42]. From the nature of exponentials and Bessel functions, we know that a physical solution requires that in the first and last medium $A_{N}=B_{1}=0$, so we are left with $2 N-2$ unknown coefficients. The next step is to substitute eq. (1.10) or eq. (1.11) into eq. (1.8) to obtain the fields in each medium. The $2 \mathrm{~N}-2$ unknown coefficients can then be found by imposing the continuity of the tangential components of the fields on each of the $N-1$ interfaces $^{13}$. A linear system of $2 N-2$ equations is thus obtained.

Since electromagnetic modes have a given amplitude, we expect that any solution admits an arbitrary scaling factor. This requires the coefficient matrix of the linear system of equations to be singular. This condition yields the dispersion relation of the structure, the valid points $\left(\omega, \gamma_{z}\right)$ in which a mode exists.

\footnotetext{
${ }^{11}$ The most general solution would be $A_{z i}(\rho, \phi, z)=\left[A_{i} I_{m}\left(\gamma_{\rho i} \rho\right)+B_{i} K_{m}\left(\gamma_{\rho i} \rho\right)\right]\left[C_{i} e^{i m \phi}+D_{i} e^{-i m \phi}\right]$ $\left[E_{i} e^{\gamma_{z} z}+F_{i} e^{-\gamma_{z} z}\right]$, but assumptions similar to those for planar slabs can be used to simplify this.

${ }^{12}$ Without loss of generality, we use modified Bessel functions, which are evanescently decaying, unlike regular Bessel functions. This is analogous to using exponentials instead of trigonometric functions.

${ }^{13}$ In conventional cylindrical dielectric waveguides (e.g. optic fibers), the modes are hybrid - not purely $\mathrm{TE}_{z}$ or $\mathrm{TM}_{Z}$ - so we have to add up the fields coming from both vector potentials $\mathbf{A}=A_{z} \hat{\mathbf{z}}$ and $\mathbf{F}=F_{z} \hat{\mathbf{z}}$ before applying the boundary conditions. In plasmonic modes however, it still holds that modes are $\mathrm{TM}_{Z}$, in which only the field components $E_{z}, E_{\rho}$ and $H_{\phi}$ are non-zero.
} 


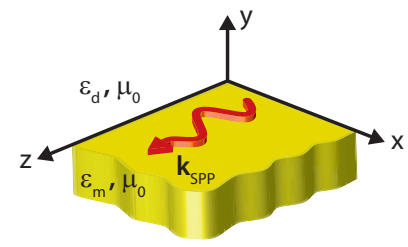

Figure 1.4: Surface plasmon propagating along the surface of a metal

The whole procedure can be easily automated in a mathematical software. A good technique is to use singular value decomposition of the system matrix to obtain the unknown coefficients. The dispersion relations and field distributions can be calculated in a very fast way. Plasmons in planar waveguides can be obtained in various layers of alternating metals and dielectrics, with very interesting properties [43], and plasmons in cylindrical nanowires can also be found [44]. Our work [PAPER A, PAPER C, PAPER G] all make extensive use of this method, which we implemented to calculate plasmonic modes in cylindrical and planar geometries.

\subsection{Surface plasmon in a smooth metal interface}

The simplest plasmonic geometry is shown in Fig. 1.4, depicting the surface of a metal with relative permittivity $\varepsilon_{m}(\omega)$ in a dielectric environment of relative permittivity $\varepsilon_{d}$. Following the procedure of the last section, we can find a solution corresponding to a surface plasmon polariton, whose electromagnetic fields are:

$$
\left\{\begin{array}{l}
H_{x}(\mathbf{r})=A e^{i k_{z} z} e^{-\alpha_{d} y} \\
E_{z}(\mathbf{r})=\frac{i A \alpha_{d}}{\omega \varepsilon_{0} \varepsilon_{d}} e^{i k_{z} z} e^{-\alpha_{d} y} \\
E_{y}(\mathbf{r})=-\frac{A k_{z}}{\omega \varepsilon_{0} \varepsilon_{d}} e^{i k_{z} z} e^{-\alpha_{d} y}
\end{array}\right\} \text { for } y>0 \text { and }\left\{\begin{array}{l}
H_{x}(\mathbf{r})=A e^{i k_{z} z} e^{\alpha_{m} y} \\
E_{z}(\mathbf{r})=-\frac{i A \alpha_{m}}{\omega \varepsilon_{0} \varepsilon_{m}} e^{i k_{z} z} e^{\alpha_{m} y} \\
E_{y}(\mathbf{r})=-\frac{A k_{z}}{\omega \varepsilon_{0} \varepsilon_{m}} e^{i k_{z} z} e^{\alpha_{m} y}
\end{array}\right\} \text { for } y<0,
$$

where $\mathrm{A}$ is the amplitude of the mode, $k_{z}$ is the propagation constant of the mode $\mathrm{e}^{14}$ and $\alpha_{m, d}$ is the attenuation constant ${ }^{15}$ towards $y$. The dispersion relation of the mode is given by:

$$
k_{z}=k_{0} \sqrt{\frac{\varepsilon_{d} \varepsilon_{m}}{\varepsilon_{d}+\varepsilon_{m}}} \quad \text { with } \quad k_{0}=\omega \sqrt{\mu_{0} \varepsilon_{0}} .
$$

Figure 1.6(a) plots the dispersion relation $k_{z}$ vs. frequency $\omega=2 \pi f$ for an $\varepsilon_{m}(\omega)$ given by a lossless Drude model. The bound nature of the surface plasmon can be

\footnotetext{
${ }^{14}$ We defined $\gamma_{z}=-\alpha_{z}+i k_{z}^{\prime}$, but here we allow $k_{z}=k_{z}^{\prime}+i \alpha_{z}$ to be complex, so that $\exp \left(\gamma_{z} z\right)=\exp \left(i k_{z} z\right)$

${ }^{15}$ Again, we defined $\gamma_{y}=-\alpha_{y}^{\prime}+i k_{y}$ but we allow a complex $\alpha_{y}=\alpha_{y}^{\prime}-i k_{y}$ so that $\exp \left(\gamma_{y} y\right)=\exp \left(-\alpha_{y} y\right)$
} 


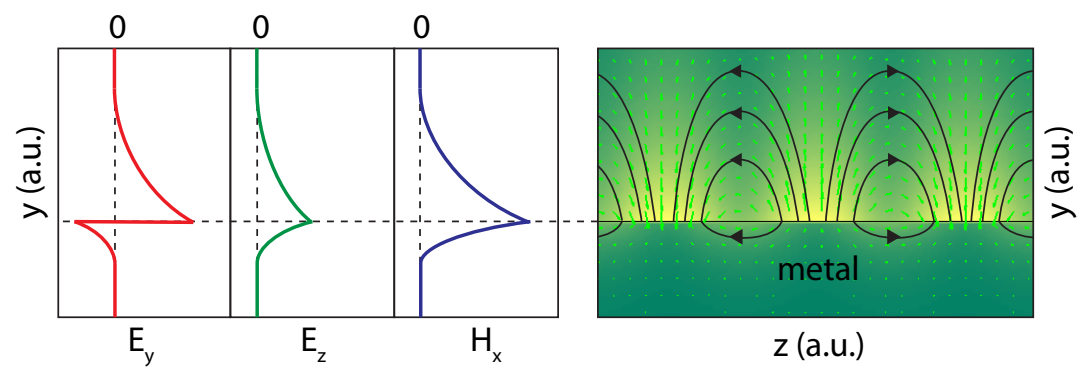

Figure 1.5: Electromagnetic fields of a surface plasmon polariton propagating in a smooth metal-dielectric interface. The discontinuity in $E_{y}$ accounts for surface charge accumulation.
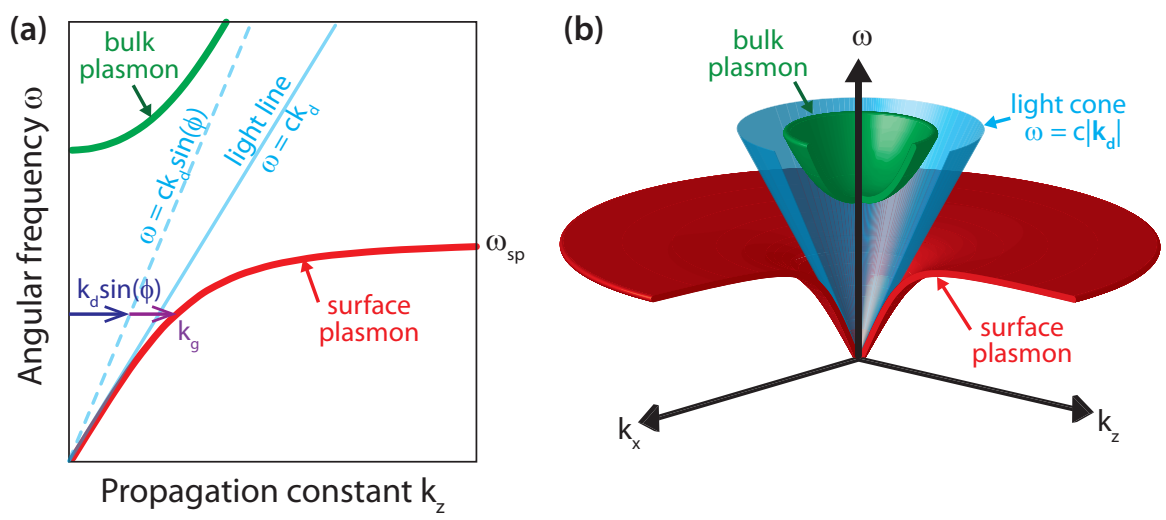

Figure 1.6: (a) Dispersion relation of a surface plasmon on a Drude metal interface with a dielectric (b) Three-dimensional representation of (a), allowing propagation in any direction of the surface 


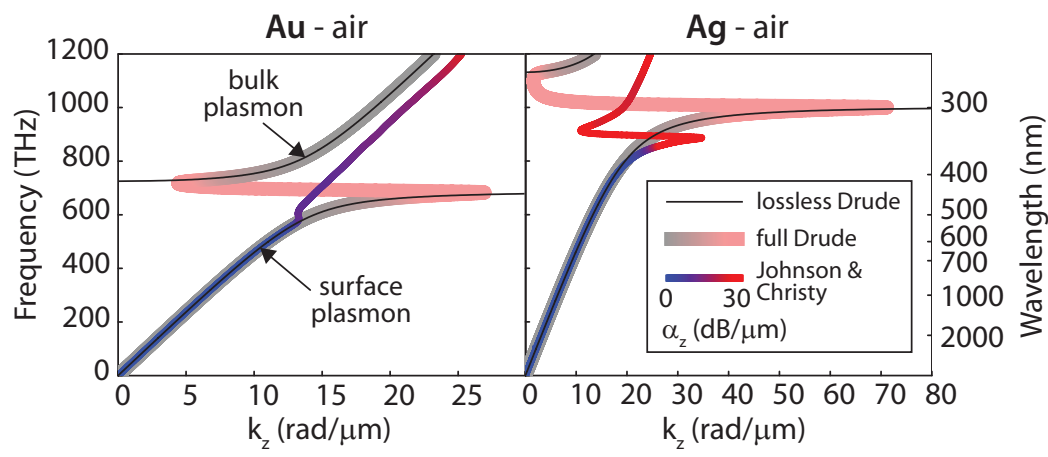

Figure 1.7: Dispersion relation for the surface plasmons at a (a) gold and (b) silver interface with air, for three different cases of metal dielectric constants $\varepsilon_{m}(\omega)$ : lossless Drude model (solid black line), lossy Drude model as given in Fig. 1.2 (thickest line) and experimental values from Johnson and Christy (medium line). For the last two cases, the color of the line indicates the propagation loss coefficient.

inferred as follows: from the constraint equation $\gamma_{y}^{2}+\gamma_{z}^{2}=-k^{2}$ we know that the propagation and attenuation constants must satisfy the relations

$$
k_{z}^{2}-\alpha_{m}^{2}=k_{m}^{2} \quad \text { and } \quad k_{z}^{2}-\alpha_{d}^{2}=k_{d}^{2} .
$$

Since $k_{m}^{2}=k_{0}^{2} \varepsilon_{m}$ is negative, then $\alpha_{m}$ is positive and real, and thus the fields decay exponentially into the metal. This explains reflection in a metal. Most importantly, if $k_{z}>k_{d}$, as occurs for the dispersion relation of the surface plasmon, then $\alpha_{d}>0$ and real, meaning that the mode also decays exponentially away from the interface into the dielectric. Therefore, any mode that lies outside of the light cone [see Fig. 1.6(b)] is confined to the surface of the metal. The confinement is stronger the bigger $k_{z}$ is, so it is particularly strong near the surface plasmon frequency $\omega_{s p}$ where the dispersion relation is asymptotic towards large $k_{z}$ because $\varepsilon_{m}(\omega)=-\varepsilon_{d}$. Confinement is very strong near this frequency and the wavelength of the surface plasmon tends to zero.

In addition, from Fig. 1.6(a) we can see the existence of bulk plasmons that propagate into the metal when $\varepsilon_{m}(\omega)>0$, which occurs at the plasma frequency $\omega_{p}$ if $\varepsilon_{\infty}=1$. Between $\omega_{s p}$ and the bulk plasmon at $\omega_{p}$, there is a region in which $k_{z}$ is purely imaginary, called a bandgap, where propagation is forbidden.

All previous results correspond to a lossless scenario. To study a realistic case, however, we should consider the imaginary part in the dielectric function of the metal, by considering the complete Drude model, or even the experimental values of a metal such as gold and silver. In both cases, the dispersion relation yields complex values for the propagation constant $k_{z}=k_{z}^{\prime}+i \alpha_{z}$, so that $\exp \left(i k_{z}\right)=\exp \left(i k_{z}^{\prime}\right) \exp \left(-\alpha_{z} z\right)$ accounts for the propagation loss. The value of $\alpha_{z}$ is called the attenuation coefficient ${ }^{16}$.

\footnotetext{
${ }^{16} \alpha(\mathrm{dB} / \mathrm{m})=20 \ln (10)[\alpha(\mathrm{Np} / \mathrm{m})] \approx 8.686[\alpha(\mathrm{Np} / \mathrm{m})]$
} 
Figure 1.7 shows the dispersion relation of a gold and silver interface with air for three cases: lossless Drude model, lossy Drude model, and experimental values from Johnson and Christy [41]. The values of the attenuation are coded in the color of the line.

\subsection{Excitation of surface plasmons}

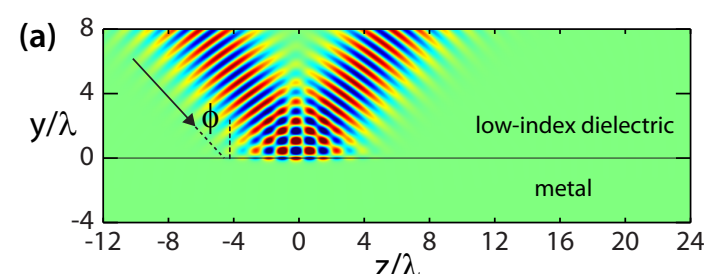

(b)
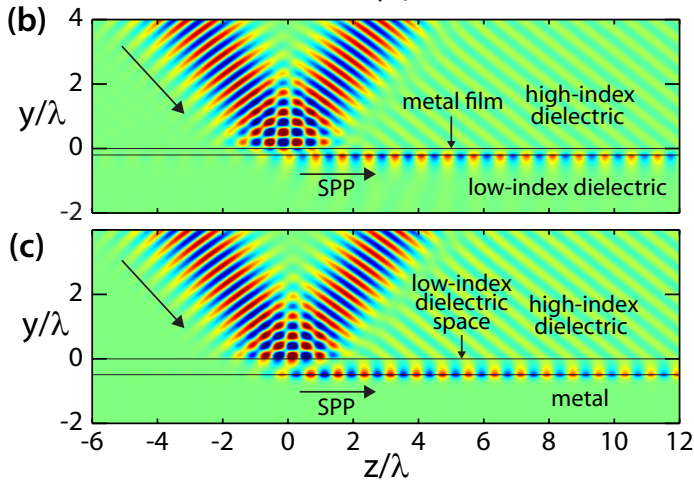
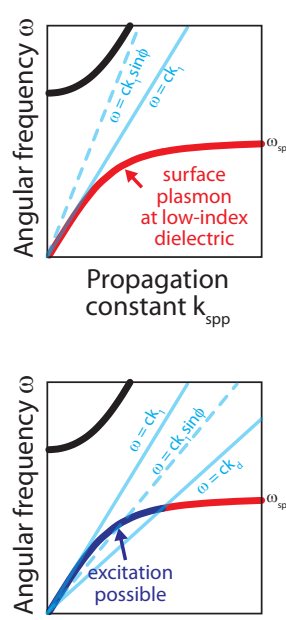

Propagation constant $\mathrm{k}_{\text {spp }}$

Figure 1.8: Prism excitation of surface plasmons. (a) Incident beam at angle $\phi$ is reflected if no prism is used. A high-index prism allows coupling to SPPs as shown in (b) Kretschmann configuration and (c) Otto configuration. Color represents instantaneous magnetic field. The field is calculated using a transfer matrix method developed during this thesis.

Consider a light beam illuminating a metal surface at an angle $\phi$, as shown in Fig. 1.8(a). We can see that all the light is reflected. Surface plasmon polaritons on a flat metal/dielectric interface cannot be excited directly by light beams because of a wavevector mismatch $k_{d} \sin (\phi) \leq k_{d}<k_{S P P}$. Therefore, special coupling techniques must be used.

- Prism coupling: makes use of the higher wavevector achievable inside a prism of high dielectric constant. This requires a three layered system, so that evanescent coupling between light incoming from the high-index prism and the surface plasmon in the low-index dielectric-metal interface can take place. Two 

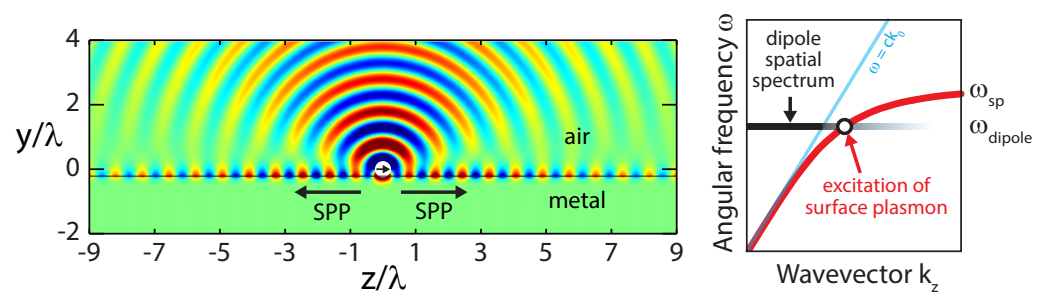

Figure 1.9: Dipole excitation of surface plasmons. In this calculation showing the instantaneous magnetic field, an horizontal electric point dipole is placed at a distance $\lambda / 4$ from a metal surface.

possible configurations [22, 45] can be used to achieve this, namely Kretschmann configuration [Fig. 1.8(b)] and Otto configuration [Fig. 1.8(c)].

- Grating coupling: by patterning the metal surface with a shallow grating of grooves or holes with a lattice constant $a$, the SPP mode sustained by it is no longer a single wavevector $k_{S P P}$ but instead becomes a Bloch mode with multiple wavevectors at $k_{S P P} \pm n \frac{2 \pi}{a}$ with $n \in \mathbb{N}$. This is equivalent to adding a grating wavevector $k_{g}=n \frac{2 \pi}{a}$ to the in-plane momentum of the incident light, acquiring the necessary in-plane momentum for coupling to take place as depicted in Fig. 1.6(a).

- Near-field excitation: while $k_{d} \sin (\phi) \leq k_{d}<k_{S P P}$ is always true for propagating light in the dielectric medium, the evanescent components of near field sources can have in-plane wave-vector components of arbitrary magnitude, and thus excite SPPs [46]. However, these components decay exponentially when we move away from the source, i.e. they only exist in the near field, at distances $d<\lambda$. Fig. 1.9 shows a source point dipole oscillating at a distance $\lambda / 4$ from a metal surface, achieving excitation of SPPs. In practice this point dipole can be experimentally realized by a small probe tip, a small aperture, or by the scattering from a small particle or surface discontinuity. 


\section{Chapter 2}

\section{Negative index metamaterials}

IN THIS CHAPTER, a brief introduction to metamaterials is made, followed by a description of negative index of refraction and its most famous application, the perfect lens. This will serve as presentation for our two papers [PAPER A] and [PAPER B], describing the theory of a negative index plasmonic metamaterial and the experimental observation of the discussed resonances. Later, a brief description of two-dimensional negative index lenses based on plasmonic waveguides will be given, of which we propose an extension of the concept to three-dimensions, resulting in a metamaterial composed of coaxial plasmonic waveguides [PAPER C].

\subsection{Metamaterials - what are they?}

Metamaterials are engineered composites that exhibit superior properties not found in nature and not observed in their constituent materials. The search for a less general definition of the term has some difficulties, due to the great variety of contexts in which metamaterials are used (see discussion in [47, 48]).

To understand metamaterials, we must first understand conventional materials, which owe their electromagnetic properties to the atoms and molecules of which they are composed. Light, as any other electromagnetic wave, interacts with those atoms and undergoes scattering ${ }^{1}$. As a result, the electromagnetic fields $\mathbf{E}$ and $\mathbf{B}$ have microscopic fluctuations within matter, and two distinct regimes ${ }^{2}$ can then be identified [48]:

- If the wavelength of light is comparable to the periodicity of the material atomic or molecular structure, Bragg effects [49] come into play. This is the basis of $\mathrm{x}$-ray diffraction and electron diffraction in crystals.

\footnotetext{
${ }^{1}$ Scattering is caused by the electromagnetic field exerting forces and causing movement in charges, which in turn generate a new electromagnetic field.

${ }^{2}$ Although the threshold between them is not well defined.
} 
- If the wavelength of light is much bigger than the periodicity of the material, then the local fluctuations in $\mathbf{E}$ and $\mathbf{B}$ can be averaged over space, and the effect of the material can be summarized into the average polarization $\mathbf{P}$ (density of electric dipoles) and magnetization $\mathbf{M}$ (density of magnetic dipoles) vectors, which appear in the macroscopic Maxwell equations (see section 1.2.1 in chapter 1) without having to worry about internal polarization currents and charges $[36,50,51]$. This process is called homogenization or effective medium theory.

While both Bragg effects $[49,52]$ and homogenization $[53,54]$ were very well known to physicists during the $20^{\text {th }}$ century, the idea of applying them to artificial atoms or man-made structures which can also scatter light, in order to design advanced materials [55], had to wait until the late part of the century. The analogous two regimes can be defined:

- When man-made structures are ordered with separations comparable to the wavelength, we have a photonic crystal [56,57], which allows exciting possibilities such as bandgaps and slow wave phenomena. In a periodic crystal the fields are expressed as Bloch waves, with a periodic envelope, associated to a summation of multiple wave vectors.

- When the man-made structures - also called meta atoms - have a periodicity much smaller than the wavelength, then the zeroth order Bloch wave dominates, and we can apply a second ${ }^{3}$ homogenization process, considering the whole collection of artificial scatterers as a homogeneous continuous material with an effective polarization $\boldsymbol{P}_{\text {eff }}$ and magnetization $\mathbf{M}_{\text {eff }}$ (see Fig. 2.1) which depends not only on the constituent materials, but also on the geometry of the artificial meta atoms. This constitutes a metamaterial.

In a very general case, the polarization and magnetization of a material or metamaterial can be very complex functions of the incident fields. Let us consider linear materials - a common assumption for low energy fields, but leaving behind the interesting field of nonlinear electromagnetics.- For linear materials, using the definition of $\mathbf{D}$ and $\mathbf{H}$ [see eqs. (1.2a) and (1.2b)] we can write the following constitutive relations ${ }^{4}$, neglecting the electric quadrupole and higher moments:

$$
\begin{aligned}
& \mathbf{D}(\mathbf{k}, \omega)=\overbrace{\varepsilon_{0} \mathbf{E}+\mathbf{P}_{E}}^{\varepsilon_{0} \varepsilon \mathbf{E}}+\overbrace{\mathbf{P}_{H}}^{-i c_{0}^{-1} \xi \mathbf{H}}, \\
& \mathbf{B}(\mathbf{k}, \omega)=\underbrace{\mu_{0} \mathbf{H}+\mu_{0} \mathbf{M}_{H}}_{\mu_{0} \mu \mathbf{H}}+\underbrace{\mathbf{M}_{E}}_{i c_{0}^{-1} \xi^{t} \mathbf{E}} .
\end{aligned}
$$

\footnotetext{
${ }^{3}$ Meta atoms are composed, obviously, from natural materials, so a first homogenization of the fields on such materials has to be performed first.

${ }^{4}$ Linearity allows us to work with the spatial and temporal Fourier transform of fields
} 


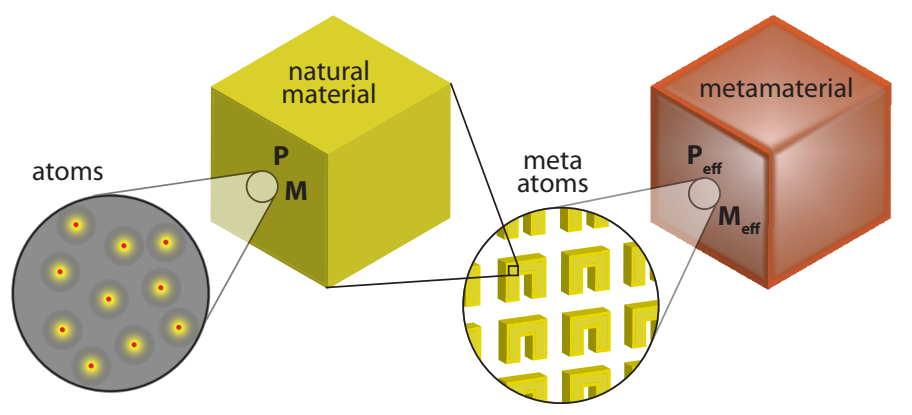

Figure 2.1: Analogy between the atoms in a material causing a homogenized polarization $\mathbf{P}$ and magnetization $\mathbf{M}$, and the meta atoms in a metamaterial causing a secondly homogenized polarization $\mathbf{P}_{\text {eff }}$ and magnetization $\mathbf{M}_{\text {eff }}$.

Here, the polarization and magnetization was split according to its physical origin ${ }^{5}$. All the properties of linear homogeneous matter can then be summarized into three parameters: the relative permittivity $\varepsilon(\mathbf{k}, \omega)$ related to electric polarizability, the relative permeability $\mu(\mathbf{k}, \omega)$ related to magnetic polarizability and the bianisotropy [58] factor $\xi(\mathbf{k}, \omega)$. In general, the three parameters are tensors because the properties of the material can depend on the polarization direction of the fields.

In many cases the situation is simpler: bianisotropy is often neglected $(\xi \approx 0)$, materials are isotropic so that $\varepsilon$ and $\mu$ are complex scalars rather than tensors, and the spatial dispersion -implied by the wavevector dependence- is negligible ${ }^{6}$. Thus, under such assumptions, all the properties of the material can be modeled completely by the two parameters $\varepsilon(\omega)$ and $\mu(\omega)$.

The availability of materials, and therefore the availability of combinations of $\varepsilon$ and $\mu$, are limited in nature. Metamaterials provide us the required freedom to design appropriate meta atoms, such that the effective $\varepsilon$ and $\mu$ can have values designed at will.

Although artificial unit cells to control electromagnetic fields had been occasionally used throughout the $20^{\text {th }}$ century in different contexts [59-62], it was the relatively recent work of J. Pendry and coworkers $[63,64]$ which spawned interest in metamaterials, and the number of publications has grown exponentially since then. For a more comprehensive historical review of the field, see Refs. [48, 65]. The current advances in nanotechnology and nanofabrication open the door to the possibility of constructing sub-wavelength meta atoms even at optical wavelengths, to achieve unprecedented values of material properties $\varepsilon$ and $\mu$, including negative values.

\footnotetext{
${ }^{5}$ Normally an electric field $\mathbf{E}$ induces electric dipoles $\mathbf{P}_{E}$ in the material, while a magnetic field $\mathbf{B}$ induces magnetic dipoles $\mathbf{M}_{H}$ in the material, but a behavior known as bianisotropy allows magnetic fields to generate electric dipoles $\mathbf{P}_{H}$, and vice versa $\mathbf{M}_{E}$.

${ }^{6}$ This allows us to avoid the spatial Fourier transform and work only with time domain transformed fields $\mathbf{E}(\mathbf{r}, \omega)$, equivalent to phasor notation of a harmonic field $\mathbf{E}(\mathbf{r})$.
} 


\subsection{Negative index media}

The propagation of plane waves in a homogeneous, linear, isotropic medium, is given as:

$$
\mathbf{E}=\mathbf{E}_{0} e^{i \mathbf{k} \cdot \mathbf{r}-i \omega t} \quad \text { with } \mathbf{k}=k \hat{\mathbf{k}}=n \frac{\omega}{c} \hat{\mathbf{k}},
$$

where $n=\sqrt{\varepsilon \mu}$ is called the refractive index, $c_{0}$ is the speed of light, $\mathbf{k}$ is the wavevector, whose magnitude $k$ is the wavenumber, and the unit vector $\hat{\mathbf{k}}$ is defined in the direction of energy propagation $(\mathbf{P}=\mathbf{E} \times \mathbf{H})$. The phase velocity of such a wave is given by $\mathbf{v}_{p}=\hat{\mathbf{k}} \cdot c / n$. We can consider the following scenarios, summarized in Fig. 2.2

- In a conventional medium $(\varepsilon>0, \mu>0)$, the refractive index is a positive number, affecting the phase velocity and the wavelength of light. This gives origin to the refraction of light beams. Vectors $\mathbf{E}, \mathbf{H}$ and $\mathbf{k}$ form a right-handed triplet.

- If one of the material parameters is negative, as in electric $(\varepsilon<0, \mu>0)$ or magnetic $(\varepsilon>0, \mu<0)$ plasmas, then the electromagnetic field cannot propagate. The refractive index $n=\sqrt{\varepsilon \mu}$ is imaginary, therefore $k$ is imaginary, and the complex exponential in eq. (2.2) turns into exponential decay.

- The possibility of a medium with both a negative permittivity and permeability $(\varepsilon<0, \mu<0)$ was not considered theoretically until 1968, when Veselago [66] proved convincingly ${ }^{7}$ that in such a medium not only propagation is possible, but the vectors $\mathbf{E}, \mathbf{H}$ and $\mathbf{k}$ form a left-handed triplet, so that the phase advance is opposite to the energy flowing out of the source. According to our definitions in eq. (2.2), this is equivalent to a negative index $n<0$, and no natural media was known with such property.

The properties of the so-called left-handed media ${ }^{8}$ (LHM) or negative-index media (NIM) are numerous and exceedingly surprising. There is a reversal of the Doppler effect and the Čerenkov radiation [66]. A reverse Goos-Hänchen effect that allows light trapping in NIM-core waveguides [67]. There is an exchange in the behavior of concave and convex lenses, and NIM lenses can be designed with lower aberrations [68]. There is a reversal of the Casimir force, from attraction to repulsion, enabling quantum levitation [69]. The phenomenon of negative refraction occurs between a RHM and a LHM, shown in Fig. 2.3, in accordance to Snell's law of refraction $n_{1} \sin \left(\theta_{1}\right)=n_{2} \sin \left(\theta_{2}\right)$ which still holds valid, and leads to the possibility of a planar lens. Interestingly, such NIM planar lens, which Veselago himself suggested in his seminal paper [66], was what most excited and stirred the scientific community when J. Pendry predicted its behavior as a perfect lens [8].

\footnotetext{
${ }^{7}$ Substituting eq. (2.2) into Maxwell's equations, one obtains that $\mathbf{k} \times \mathbf{H}=\omega \varepsilon_{0} \varepsilon \mathbf{E}$ and $\mathbf{k} \times \mathbf{E}=-\omega \mu_{0} \mu \mathbf{H}$

${ }^{8}$ In opposition to conventional right-handed media (RHM).
} 




Figure 2.2: Permeability and permittivity of different materials. This simplified representation is valid for lossless, linear, isotropic, homogeneous media. At the axes of this graph also lie the peculiar $\varepsilon$-near-zero and $\mu$-near-zero materials.
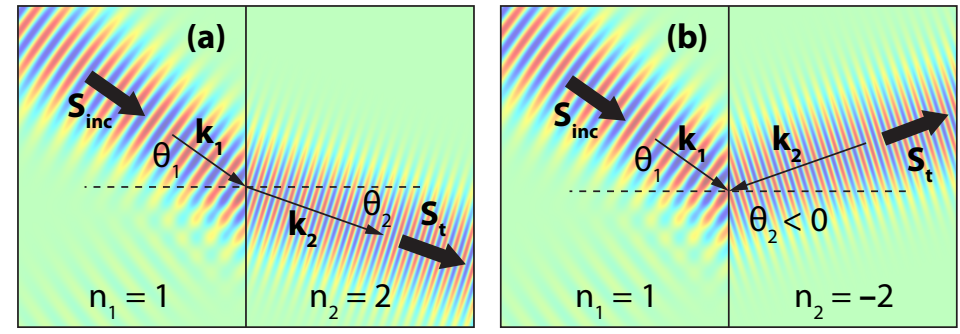

Figure 2.3: (a) Conventional refraction between right-handed media. (b) Negative refraction at the interface between a right-handed and a left-handed medium. 


\subsection{The perfect lens}

According to wave optics, no lens can focus light onto an area smaller than a square wavelength, because such fine details are not propagating but evanescent. A NIM lens, however, amplifies the evanescent components, restoring the source at the image plane with unlimited resolution [8]. This can be easily understood by considering the angular spectrum decomposition $[14,48]$ of a given monochromatic field distribution ${ }^{9}$ $A(x, y)$ at a certain plane (e.g. the source plane)

$$
A(x, y)=\iint_{-\infty}^{\infty} \hat{A}\left(k_{x}, k_{y}\right) e^{i k_{x} x} e^{i k_{y} y} d k_{x} d k_{y}
$$

which corresponds to a spatial Fourier transform with spatial frequency components $k_{x}$ and $k_{y}$. This can be interpreted as decomposing the field into a sum of plane waves $^{10}$ of amplitude $\hat{A}=\hat{A}\left(k_{x}, k_{y}\right)$, each plane wave given by:

$$
A_{p w}(\mathbf{r}, t)=\hat{A} e^{i \mathbf{k} \cdot \mathbf{r}-i \omega t} \quad \text { with } \quad \begin{aligned}
\mathbf{k} & =k_{x} \hat{\mathbf{x}}+k_{y} \hat{\mathbf{y}}+k_{z} \hat{\mathbf{z}} \\
& =(\omega \sqrt{\mu \varepsilon}) \cdot \hat{\mathbf{k}}=k \cdot \hat{\mathbf{k}} .
\end{aligned}
$$

It follows that $k_{z}=\sqrt{k^{2}-k_{x}^{2}-k_{y}^{2}}$, so we can distinguish two different regimes:

- Propagating components: When $k_{x}^{2}+k_{y}^{2}<k^{2}$, then $k_{z}$ is mainly ${ }^{11}$ real, and the plane wave is propagating in a direction $\mathbf{k}$. Conventional lenses focus these components by restoring their phase at the image plane.

- Evanescent components: When $k_{x}^{2}+k_{y}^{2}>k^{2}$, then $k_{z}$ is mainly imaginary, and these plane wave components decay exponentially in the $z$ direction. They correspond to the fine details of the image. Conventional lenses loose these components, however, the perfect lens does not.

To demonstrate the behavior of the perfect lens, we can apply the concept of the transfer function $\hat{T}\left(k_{x}, k_{y}, z\right)$, a complex function that relates each plane wave amplitude and phase at a plane $z$ with that at $z=0$

$$
\hat{A}\left(k_{x}, k_{y}, z\right)=\hat{A}\left(k_{x}, k_{y}, 0\right) \cdot \hat{T}\left(k_{x}, k_{y}, z\right),
$$

Obtaining the transfer function for a given geometry is an elementary exercise. For example, in a homogeneous medium it follows from eq. (2.4) that $\hat{T}\left(k_{x}, k_{y}, z\right)=e^{i k_{z} z}$. The transfer matrix method [70] is a powerful method for the general case of $\mathrm{N}$ slabs ${ }^{12}$.

\footnotetext{
${ }^{9} A$ can represent any component of either the electric field $\mathbf{E}$ or the magnetic field $\mathbf{H}$

${ }^{10}$ In general the source will be separated into $\mathrm{TE}_{z}$ plane waves, that can be characterized by their tangential $\mathbf{E}$ field, and $\mathrm{TM}_{z}$ plane waves, characterized by their tangential $\mathbf{H}$ field.

${ }^{11}$ In general $\varepsilon, \mu$ and thus $k$ and $k_{z}$ are complex.

${ }^{12} \mathrm{~A} 2 \mathrm{D}$ and 3D transfer matrix method was implemented as part of this thesis, and was extensively used throughout. Figs. 1.8, 1.9, 2.3, 2.4, 5.3 and 5.4 were computed using that method.
} 

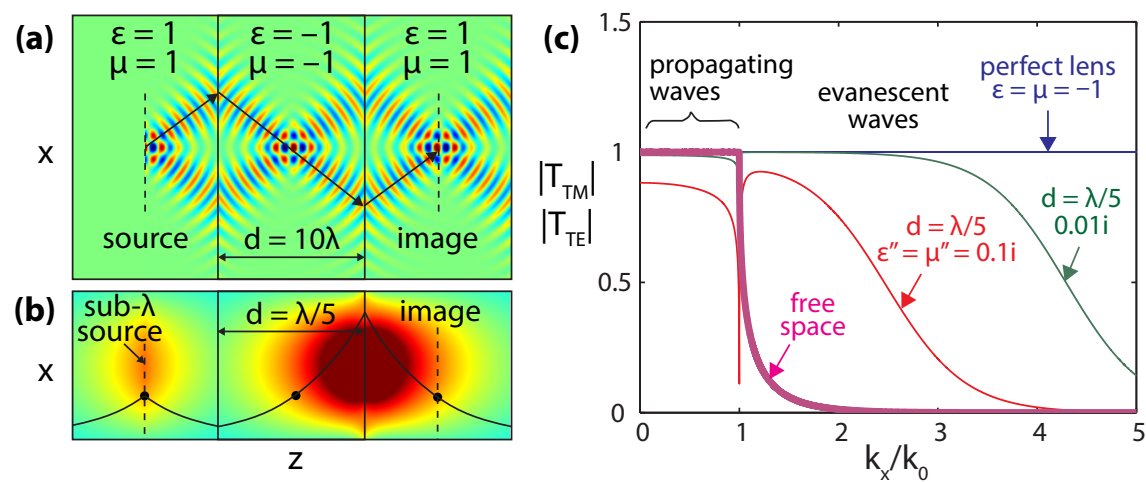

Figure 2.4: Two dimensional transfer matrix method simulation of: (a) Focusing of propagating TM beams undergoing negative refraction in a perfect lens (instantaneous magnetic field). (b) Amplification of evanescent components from a subwavelength TM source (magnetic field amplitude). (c) Absolute value of the transfer function of a perfect lens from the source to the image plane, in the ideal case, and with losses $\varepsilon=\mu=-1+\delta i$.

For a single slab of width $d$ and material parameters $\varepsilon_{2}$ and $\mu_{2}$, placed in a medium with $\varepsilon_{1}$ and $\mu_{1}$, the transfer function for the electric field of $\mathrm{TE}_{z}$ modes is $[8,48]$ :

$$
\hat{T}_{T E}=\frac{2 \mu_{2} k_{z 1}}{\mu_{2} k_{z 1}+\mu_{1} k_{z 2}} \cdot \frac{2 \mu_{1} k_{z 2}}{\mu_{2} k_{z 1}+\mu_{1} k_{z 2}} \cdot \frac{e^{i k_{22} d}}{1-\zeta^{2} e^{2 i k_{z 2} d}} \quad \text { with } \quad \zeta=\frac{\mu_{1} k_{z 2}-\mu_{2} k_{z 1}}{\mu_{1} k_{z 2}+\mu_{2} k_{z 1}}
$$

and that of $\mathrm{TM}_{z}$ modes is the same but changing $\mu_{i} \rightarrow \varepsilon_{i}$. Remarkably, when the outer medium is air $\left(\varepsilon_{1}=\mu_{1}=1\right)$, it follows that

$$
\lim _{\substack{\varepsilon_{2} \rightarrow-1 \\ \mu_{2} \rightarrow-1}} \hat{T}_{T E}=\lim _{\substack{\varepsilon_{2} \rightarrow-1 \\ \mu_{2} \rightarrow-1}} \hat{T}_{T M}=e^{-i k_{z 1} d}
$$

meaning that a NIM slab with $\varepsilon=\mu=-1$ exactly compensates an equal thickness of vacuum [8]: It causes an opposite phase propagation —as expected from the negative phase advance [Fig. 2.4(a)] — and amplifies the evanescent waves [Fig. 2.4(b)].

The amplification of evanescent waves does not violate physical laws, because they do not carry energy. Yet, it is a highly un-intuitive result responsible for perfect focusing. The effect can actually be explained as surface plasmon modes excited on the rear side of the lens. The perfect lens, also called superlens, was named one of the 10 greatest scientific achievements of the year 2003 according to the journal Science [71]. 


\section{Practical limitations}

The perfect lens was not easily accepted in the scientific community. Perfect imaging requires a negative $\left(n^{\prime}<0\right)$ and lossless $\left(n^{\prime \prime}=0\right)$ value of the refractive index. The former requirement entails a dispersive refractive index $n(\omega)$ which limits the bandwidth. The latter condition demands no absorption and in practice is never fulfilled, leading to decreased performance as shown in Fig. 2.4(c), reducing drastically the resolution [72,73], specially in thicker lenses [74]. The finite periodicity required in the fabrication of any metamaterial introduces spatial dispersion at high wavevectors, and also imposes a lower limit to the resolution [75]. Even in the lossless case the lens is sensitive to perturbations in the material properties [76]. Nevertheless, it is agreed that a NIM lens can improve imaging if its thickness is subwavelength [77]. Indeed, some years after its proposal, the first experimental results finally proved subwavelength resolution in NIM [78-80] at microwave frequencies. Furthermore, in the electrostatic limit the electric and magnetic fields are uncoupled, which allows the construction of near-perfect lenses with only one negative parameter: A metal slab with $\varepsilon<0$ can act as a superlens in the optical near field $[5,29,30]$ (sometimes called poor man's lens) and a magnetoinductive lens [81,82] with only $\mu<0$, can also act as a lens for magnetic sources, with applications in magnetic resonance imaging [83].

The concept of the hyperlens was later proposed [84, 85], which magnifies the near field of an image [86], allowing it to be focused with conventional optics. It was experimentally realized with a multilayer structure [87]. Nevertheless, achieving subwavelength resolution with a truly negative index metamaterial lens remains elusive in the optical range.

\subsection{State of the art}

Since the proposal of the perfect lens in 1999, enormous efforts were dedicated towards building negative index metamaterials. To achieve a NIM, it is generally considered that we must satisfy $\varepsilon^{\prime}<0$ and $\mu^{\prime}<0$ simultaneously, but when we have complex $\varepsilon=\varepsilon^{\prime}+i \varepsilon^{\prime \prime}$ and $\mu=\mu^{\prime}+i \mu^{\prime \prime}$, the strictly necessary condition is given by [88] $\varepsilon^{\prime} \mu^{\prime \prime}+\varepsilon^{\prime \prime} \mu^{\prime}<0$. However, the case $\varepsilon^{\prime}<0$ and $\mu^{\prime}<0$-called a double negative metamaterial - is desirable for low losses.

Smith et al. [89] were the first to experimentally fabricate and test a negative index metamaterial in 2000, working for two dimensional propagation of TE waves. The metamaterial is shown in Fig. 2.5(a), and its unit cell can be seen to have two distinct elements:

- Metal wires to achieve $\varepsilon_{\text {eff }}<0$. A wire medium metamaterial [see Fig. 2.5(b)] shows effectively the electric response of a free electron plasma (Drude response, see section 1.2.2) with a reduced plasma frequency, when the incident electric field is parallel to the wires, as demonstrated in Ref. [63]. 

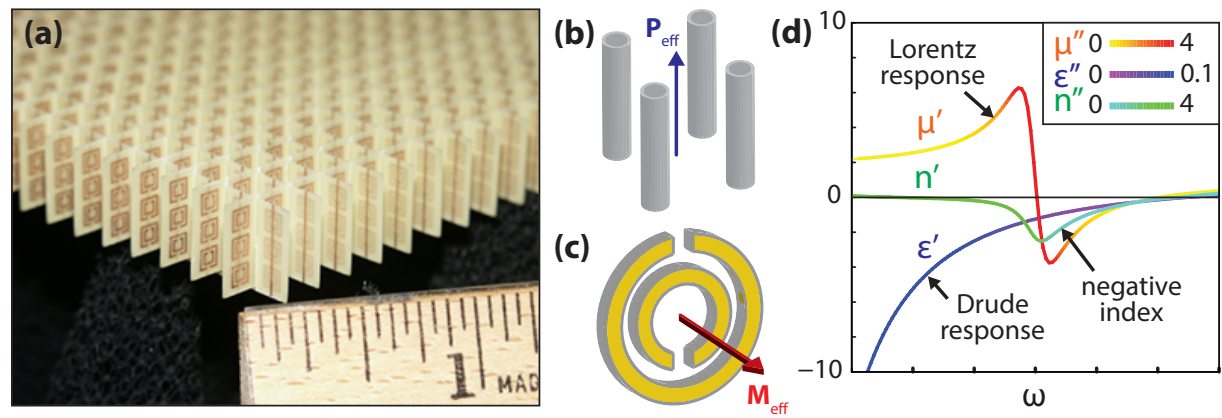

Figure 2.5: (a) Negative index metamaterial demonstrated by Smith et al. [89]. Source: NASA Glenn Research (b) A wire medium metamaterial. (c) A split-ring resonator. (d) Typical values of $\varepsilon(\omega)$ following a Drude response, $\mu(\omega)$ following a Lorentz response, and the associated $n(\omega)$.

- Split-ring resonators (SRRs) to achieve $\mu_{\text {eff }}<0$. A SRR consists of one or two concentric metallic rings that are split with a gap [see Fig. 2.5(c)]. It is a subwavelength resonator thanks to the resonance arising not from bouncing of waves between reflectors, but from parts of the metallic structure being identified as a capacitance or inductance. The resonance of a SRR produces a virtual current loop (VCL) ${ }^{13}$ which induces a magnetic dipole. If this magnetic dipole is oriented parallel to the incident magnetic field which induced it, a Lorentz response $^{14}$ in the appropriate tensor component of the effective permeability $\mu_{\text {eff }}(\omega)$ is obtained, as shown theoretically in Ref. [64]. Using SRRs is one of the most common methods to achieve negative permeability in metamaterials and their behavior has been studied in great depth [90-94]. They present bianisotropy [58] and many modifications of the basic structure have been proposed e.g. [95-98] among many others (see [48]).

The combination of both meta atoms leads to a negative index, as shown in Fig. 2.5(d). Many methods to obtain the effective $\mu$ and $\varepsilon$ of a fabricated or simulated metamaterial are available (e.g. [99-102] among others). The one most used in the literature relies on the measurement of amplitude and phase transmission and reflection through a slab [103-105]. The method was traditionally used to measure material properties [106, 107], and when applied to metamaterials should be interpreted with some precautions [108-111]. The direct measurement of a negative angle of refraction on a prism of the material is also typically used as a confirmation of a NIM.

\footnotetext{
${ }^{13} \mathrm{~A}$ virtual current loop is a loop of electric current $\mathbf{J}$ which is closed at the gaps by a displacement current $\partial \mathbf{D} / \partial t$. Both terms contribute to a magnetic field, as predicted by Ampere's law.

${ }^{14}$ If we add a restoring force $-k x$ to the Drude model, $m \ddot{\mathbf{x}}=-m \gamma \dot{\mathbf{x}}-k \mathbf{x}-e \mathbf{E}$, we obtain a Lorentz response $\varepsilon(\omega)=1-\omega_{p}^{2} /\left(\omega^{2}-\omega_{0}^{2}+i \gamma \omega\right)$ with resonant behavior at $\omega_{0}=\sqrt{k / m}$ and the possibility of negative values just above it. A similar expression can be achieved for $\mu$ with a magnetic resonator.
} 

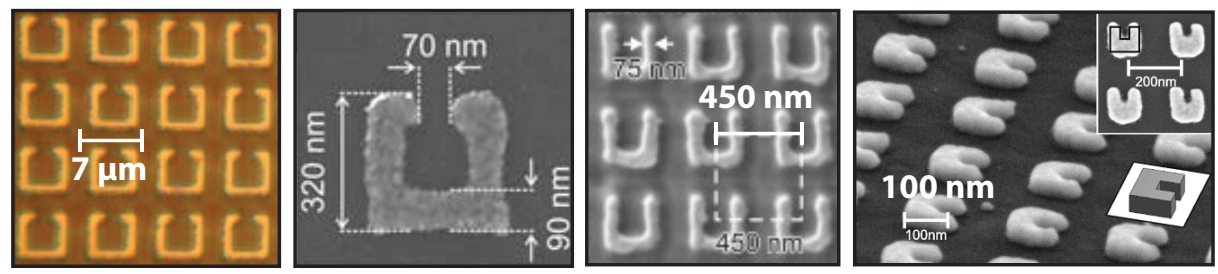

Figure 2.6: Scaling of SRRs to higher frequencies (from left to right) at $6 \mathrm{THz}, 100 \mathrm{THz}$, $250 \mathrm{THz}$, and $330 \mathrm{THz}$. Taken from [92], [112], [114] and [115], respectively.

\section{Towards optical frequencies}

Once NIM were measured in the microwave regime, a race begun towards making them work at optical frequencies, where the applications seem most interesting. Negative $\varepsilon(\omega)$ is easy to obtain at optical frequencies with the use of metals, but no natural substance is known to exhibit a magnetic response at such high frequencies. A magnetic metamaterial structure such as SRRs needs to be used.

- Scaling of SRRs: Since Maxwell's equations are invariant with scale, it was expected that miniaturizing the SRRs should rise the frequency of operation proportionally. This led to several works achieving negative permeability at increasingly higher frequencies [92, 112-115], shown in Fig. 2.6. However, the material parameters of metals are not constant in frequency, and it soon became obvious that there was a saturation in the magnetic response [116] associated with an increasing kinetic inductance of electrons [117]. At such frequencies, the resonances of the SRR can be reinterpreted as plasmonic resonances [118].

- Fishnet metamaterials: To further increase the frequency of operation, alternative structures had to be used, such as a pairs of cut-wires [119] which later evolved into the so called fishnet metamaterials. The fishnet structure consists in a stack of two or more metallic hole arrays, and it had already been proposed and tested at microwave frequencies by Beruete et al. [120], and since then has been demonstrated in microwave and millimiter frequencies [121-123]. This structure turned out to be extremely successful for achieving negative index at optical frequencies, from telecommunication wavelengths [124], up to visible frequencies [125] - a collaboration of our group with A. V. Zayats and coworkers, based on our previous theoretical predictions [Paper 9, Paper 13].For a recent review of the fabricated NIMs at optical frequencies, see Ref. [12]. A very recent unpublished work [126] reports lensing with a fishnet wide-angle NIM in the ultraviolet, albeit with no super-resolution due to loss. 
Many other structures have been proposed as negative index media at optical frequencies, such as nano-spheres [127, 128], and cut thick nano-strips [129], but to our knowledge none have been experimentally demonstrated.

\subsection{Our work: A u-shaped nanowire meta atom NIM}

As mentioned earlier, SRR resonances at optical frequencies can be interpreted from a plasmonic standpoint [118] as if the SRR was a bent nanowire. Metal nanowires are known to support propagation of highly confined surface plasmons along their length $[130,131]$ - a fact that is experimentally confirmed $[132,133]$ - and when the surface plasmons reach the ends of a short nanowire, they can be reflected and form standing wave resonances [134-136] of different orders corresponding to different resonances. When a bent nanowire is used as a metamaterial metaatom, those resonances which show a virtual current loop can create an effective magnetization $\mathbf{M}_{\text {eff }}$ and thus lead to a magnetic response [137], and those forming a dipolar distribution of charges can create a polarization $\boldsymbol{P}_{\text {eff }}$ which leads to an electric response.

- In [PAPER A], we suggest a method of bringing together in frequency two distinct standing-wave resonances of a u-shaped nanowire, one of which provides $\varepsilon_{\text {eff }}<0$ and the other $\mu_{\text {eff }}<0$, thus achieving an effective negative index with a single metaatom ${ }^{15}$. Normally, the different resonances of a nanowire exist at successive frequencies; but in this work we suggest two methods for reducing this frequency difference and bringing both resonances together, both of which resulted in an effective negative index ${ }^{16}$ :

- Insertion of a dielectric between the arms, where the magnetic resonance has a higher concentration of electric field, brings the magnetic resonance to a lower frequency, closer to the electric one.

- Arranging the nanowires with a small vertical periodicity rises the frequency of the dipolar electric resonance due to dipole-dipole coupling, bringing it closer to the magnetic resonance.

- Our second contribution [PAPER B] is an experimental work: it details the nano-fabrication and measurement, using a Fourier Transform Infra-Red (FTIR) spectrometer (see Fig. 2.7), of a sample of u-shaped nanowires. We succesfully observed the predicted resonances. In this case, the material was not a NIM, because the incident magnetic field is not exciting the virtual current loops in

\footnotetext{
${ }^{15}$ Although we were unaware of the fact at the time of publication, the use of a single metaatom to achieve simultaneous electric and magnetic responses was proposed by Saadoun and Engheta [138, 139] on $\Omega$-shaped particles, which regrettably we did not cite.

${ }^{16}$ Limited to one direction of propagation and one polarization. Also the validity of an effective medium model could be argued here because of the considerable size of the metaatoms. In addition, a formal analysis of the structure should have considered bi-anisotropy, which was not done in our paper
} 


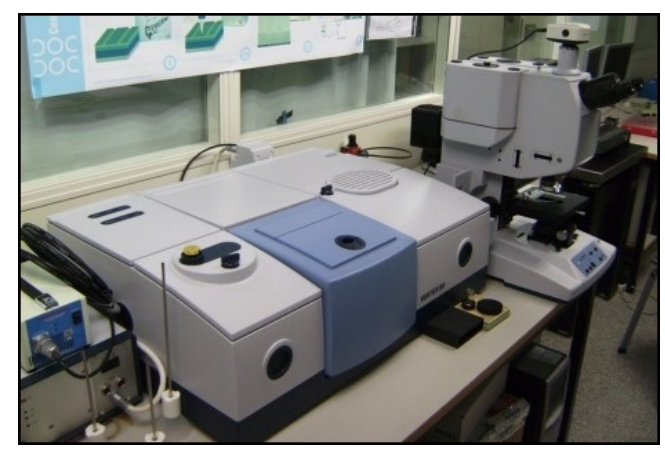

Figure 2.7: The FTIR spectrometer and its microscope, both from Bruker, used for the measurements in our works [PAPER B, PAPER F, Paper 12, Paper 16].

the nanowires, and the magnetic and electric resonances have not been brought to coincide in frequency, so this study was only a first proof of concept. We also studied numerically the negative effects that the substrate has on the excitation of the resonances.

\subsection{Backward plasmonic modes and plasmon lenses}

When two smooth metal surfaces are brought extremely close to each other at subwavelength distances, truly surprising properties can arise for the plasmons propagating between them. Consider two metals with relative permittivity $\varepsilon_{m}(\omega)$ sandwiching a dielectric of thickness $d$ with relative permittivity $\varepsilon_{d}$. The geometry is typically called a metal-insulator-metal (MIM) waveguide (see Fig. 2.8). The plasmonic modes in such waveguide can be analyzed using the method detailed in section 1.2, but we can intuitively understand their behavior with a coupling model. The plasmons propagating on the two surfaces are so close that they couple to each other, and therefore hybridize into two distinct modes: an odd mode, and an even mode, which are split in frequency. The two modes, which we name Mode I and Mode II, have the following characteristics:

- Mode I has a zero cutoff frequency and resembles the TEM mode of a parallel plate waveguide. As happens for all plasmonic modes, the wavevector grows to $\infty$ when the frequency approaches the plasma frequency $\omega_{s p}$, determined by the condition $\varepsilon_{d}=-\varepsilon_{m}(\omega)$. The dispersion relation is very similar to a single plasmon on a smooth interface, but it is lowered in frequency. 



Figure 2.8: Plasmonic modes of a MIM waveguide of different thicknesses 


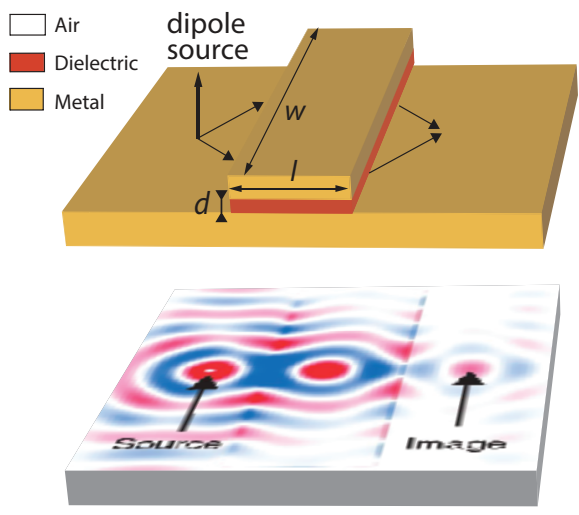

Figure 2.9: Bidimensional plasmonic lens. Taken from [142].

- Mode II has a certain cutoff frequency, which we call $\omega_{k=0}^{I I}$. The mode must be split in frequency away from Mode I, so $\omega_{k=0}^{I I}$ moves away from $\omega=0$. In fact, the splitting becomes greater as the coupling increases -i.e. as the distance $d$ between metals decreases- so, when the distance $d$ is sufficiently small, $\omega_{k=0}^{I I}$ can move above $\omega_{s p}$. This implies that the slope of the mode dispersion must be negative ${ }^{17}$. The group velocity $v_{g}=\partial \omega / \partial k$ is therefore opposite to $k$, so the effective index of the mode is negative. This was noticed in Ref. [140]. Another test for this backward behavior is that the imaginary part of $k$ has an opposite sign to the real part, meaning that the mode attenuates in the direction opposite to the phase propagation.

An alternative explanation to the backward nature of mode II can be given in terms of the Poynting vector. For any plasmonic mode, the electric field normal to the metal changes sign at the interface ${ }^{18}$. Due to this, the power flow inside the metal is opposite to the phase advance (see $P_{z}$ in Fig. 2.8). The total power flow of the mode will be given by the averaged Poynting vector over the mode profile. In Mode II, there are equal charges at opposite sides of the dielectric gap, so moving the metals together forces the electric field into the metal. If the majority of the field exists inside the metal, then the Poynting vector inside the metal dominates over that in the dielectric, and the mode becomes backward.

Yet another explanation of the phenomenon is given in terms of the nanocircuit equivalent of the MIM mode, yielding a backward transmission line [141].

\footnotetext{
${ }^{17}$ Because the wavevector goes to $\infty$ at $\omega_{s p}$, as required by any plasmonic mode

${ }^{18}$ As can be immediately derived from the boundary condition $\varepsilon_{d} E_{d}^{y}=\varepsilon_{m} E_{m}^{y}$, together with $\varepsilon_{m}<0$.
} 
The backward behavior of plasmons in MIM waveguides ${ }^{19}$ was proposed theoretically as a means of achieving a planar plasmonic lens [142] (see Fig. 2.9) capable of focusing surface plasmons after experiencing negative refraction. Soon, this was verified experimentally [144]. The use of backward MIM waveguides was later proposed as a route to achieve negative index metamaterials [145].

\subsection{Our work: NIM based on coaxial plasmonic waveg- uides}

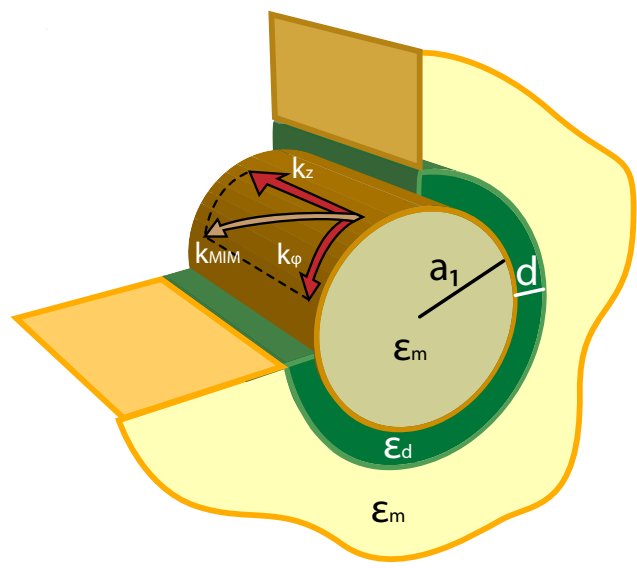

Figure 2.10: Coaxial plasmonic waveguide. The behavior is analogous to a planar MIM waveguide, but it confines light in two directions.

A disadvantage of planar MIM waveguides is that they confine light only in one direction. In our work [PAPER C] we propose using backward coaxial MIM waveguides (see Fig. 2.10), which confine light in two dimensions, to create a negative index metamaterial.

The modes of the coaxial plasmonic waveguide were rigorously studied using the method described in Section 1.2 of Chapter 1. However, to avoid mathematical details, we can intuitively understand the dispersion relation of a coaxial MIM by analogy with a planar MIM waveguide, as proposed in Ref. [146]. Consider that a planar MIM (which has a known dispersion relation $k_{M I M}$ ) is bent on itself into a cylinder. Then, the wavevector in the angular direction must verify the condition $2 \pi r k_{\phi}=m 2 \pi$, with $m \in \mathbb{N}$ in order to sustain itself constructively. This yields a dispersion relation of the coaxial waveguide equal to $k_{z}=\left(k_{M I M}^{2}-k_{\phi}^{2}\right)^{1 / 2}$ (see Fig. 2.10). This model is

\footnotetext{
[143].

${ }^{19}$ The special case in which $\omega_{k=0}^{I I}=\omega_{s p}$ was also suggested to be used for omnidirectional resonators
} 
valid as long as $a_{1} \gg d$, and in analogy with planar MIM waveguides it predicts a backward behavior for Mode II if $d$ is sufficiently small. The exact calculation of the modes confirms this result.

The existence of a backward mode in a unidimensional waveguide with such a subwavelength mode profile allows the design of a metamaterial consisting of an array of many such waveguides. An incident plane wave excites the modes of the waveguide with $m=1$, which have a backward behavior. This yields a negative effective index metamaterial at normal incidence. The results are discussed in [PAPER C]. The metamaterial has the following advantages:

- It is very sub-wavelength in the transverse direction (the diameter of the waveguide is highly sub-wavelength), which is always desirable in the design of metamaterials.

- It is uniform in the propagation direction, therefore avoiding the problem of the effects of stacking several unit cells.

- It is insensitive to incoming light polarization, since the unit cell is rotationally invariant.

- We can obtain negative index at high frequencies, above $\omega_{s p}$.

- The prediction of the index and the losses can be done analytically by using the modes of the waveguide.

The disadvantage of the metamaterial is the high losses presented by such waveguide, as studied in [PAPER C]. We also proposed the extension of the metamaterial to three dimensions by interleaving coaxial waveguides. At the time of publication, we were worried about the behavior of wave propagation in directions not parallel to the waveguides, although the excitation of waveguide modes by incoming plane waves was shown to happen for a wide range of angles. We also considered the possible existence of coupling between nearby waveguides as a potential disadvantage. However, in a work which was carried out in parallel to ours, the same geometry for a negative index was proposed [147, 148]. In addition to the NIM behavior at normal incidence at visible frequencies, they show that the negative index behavior actually occurs in a wide range of angles. This is ascribed to the coupling between waveguides, similar to the coupling in a stack of metal and insulator layers [149]. This makes the coaxial plasmonic waveguide metamaterial as one of the first proposed theoretical wide angle NIM in the visible spectrum. 


\subsection{PAPER A}

Phys. Rev. B 79, 075103 (2009)

\section{Modeling high-order plasmon resonances of a U-shaped nanowire used to build a negative-index metamaterial}

Francisco J. Rodríguez-Fortuño, Carlos García-Meca, Rubén Ortuño, Javier Martí, and Alejandro Martínez 


\title{
High-order plasmon resonances on a u-shaped nanowire as a negative- index metamaterial
}

\author{
Francisco J. Rodríguez-Fortuño, Carlos García-Meca, Rubén Ortuño, Javier Martí, and \\ Alejandro Martínez
}

Valencia Nanophotonics Technology Center, Universidad Politécnica de Valencia Campus del Camino de Vera, 46022 Valencia (Spain)

\begin{abstract}
We apply the concept of slow surface plasmon polariton standing wave resonances to model the plasmon resonances which exist on split-ring resonators (u-shaped nanowires) forming the unit cell of a metamaterial at infrared frequencies. We compare the expected resonances predicted by the model with full electrodynamic 3D simulations of the u-shaped nanowires for varying geometrical parameters and find a reasonably good agreement. We also consider how far-field dipolar coupling between unit-cells and near-field coupling between the u-shaped nanowire's arms should be taken into account. In addition, we study how the different resonances give rise to negative constitutive parameters for the metamaterial and adjust the geometrical parameters so that the second and third order slow-SPP standing wave resonances of the $u$-shaped nanowires result in a double-negative behavior at far infrared wavelengths without the need of further wires or particles. Finally, we study the effects of stacking $N$ layers of such metamaterial, where each resonant mode splits into $N$ normal mode resonances, showing different electric or magnetic responses. This simple stacked structure maintains the left-handed behaviour, exhibiting negative refraction and backward wave propagation.
\end{abstract}

\section{INTRODUCTION}

Artificially structured materials, termed metamaterials, are composed of artificial atoms much smaller than the wavelength of the incident electromagnetic field, which allow the metamaterial to be modeled by an effective homogenous medium to which constitutive electromagnetic parameters $\varepsilon_{\text {eff }}$ and $\mu_{\text {eff }}$ can de ascribed. Of particular interest are the socalled left-handed metamaterials (LHM) showing a negative index of refraction which can be produced by a negative electric permittivity $\varepsilon_{\text {eff }}$ and a negative magnetic permeability $\mu_{\text {eff }}$ at the same frequency. ${ }^{1}$ These LHMs show unusual optical properties and have the potential application of a perfect lens. ${ }^{2}$

The negative permeability $\operatorname{Re}\left(\mu_{e f f}\right)<0$ is typically achieved at microwave frequencies by using split ring resonators (SRRs). ${ }^{3}$ Considerable effort was done to scale down the SRRs to terahertz, telecommunication and optical frequencies, ${ }^{4,5}$ and at high frequencies the LC model of the SRR, valid at microwave frequencies, had to be refined. ${ }^{6}$ Finally, the LC resonance at optical frequencies was reinterpreted as a surface plasmon polariton (SPP) resonance. ${ }^{7,8}$ This gave much physical insight into the various resonances observed in SRRs at optical frequencies, which were all identified as higher order plasmon resonances, but no further details were given on the nature of these plasmon resonances. 
The so called surface plasmon polaritons (SPPs) are propagating electromagnetic waves sustained along a metal/dielectric interface, coupled to collective oscillations of the conduction electrons in the metal. ${ }^{9}$ These waves propagate highly confined to the interface, with the fields exponentially decaying away from it.

In particular, a metallic nanowire at optical frequencies allows propagation of leaky and bound SPP waves along its length. ${ }^{10-12}$ One of the bound modes supported by nanowires is the short ranging SPP (SR-SPP), also called slow-SPP, which is highly confined inside the metal, thereby slowing down the mode propagation while increasing the attenuation. If we consider metallic nanowires finite in length, one can model the end faces as reflecting the incident SR-SPP modes (but not the other modes, namely the long ranging SPPs, which show weak reflection at the ends) ${ }^{13,14}$ and turn the nanowire into a resonator ${ }^{13-18}$ showing standing slow-SPP waves when the nanowire length is approximately an integer of the half of the plasmon wavelength.


FIG. 1. (a) Unit cell of the analyzed metamaterial showing the relevant geometrical parameters and the incident plane wave. (b) Single layer metamaterial used in simulations. 
These slow-SPP standing wave resonances have been identified in optical nanoantennas, ${ }^{17}$ and can play a role in the building blocks of metamaterials at optical frequencies. ${ }^{19}$ In Ref. 7 the resonances of a SRR where associated with plasmon resonances: we bring this interpretation one step further by explicitly applying the slow-SPP standing wave nanowire resonator model to the SRR resonances. We also carry out a numerical study on the possibility of using the usually overlooked higher order slow plasmon resonances of the SRR (in particular the $2^{\text {nd }}$ and $3^{\text {rd }}$ order) to achieve electric and magnetic polarizability, and thus negative constitutive parameters.

\section{ANALYZED STRUCTURE AND NUMERICAL TOOLS}

A single unit cell of the analyzed metamaterial consists of a gold u-shaped nanowire as shown in Fig. 1, which depicts the relevant geometrical parameters. The nanowire lies in the $\mathrm{XZ}$ plane and the unit cell is repeated in the $x$ and $y$ directions with $a_{x}$ and $a_{y}$ periodicity, respectively.

Three dimensional numerical calculations were performed using a commercial electromagnetic solver (CST MICROWAVE STUDIO). A plane wave propagating in the $z$ direction with the magnetic field parallel to the $y$ axis (perpendicular to the nanostrip plane) was incident in the structure, and the transmission and reflection coefficients were obtained for a certain frequency range. Field patterns and electric currents inside the nanostrip at certain frequencies were also obtained.

For our simulations, gold's permittivity was modeled using the Drude model $\varepsilon(\omega)=\varepsilon_{\infty}-\omega_{p}{ }^{2} /\left(\omega^{2}+i \gamma \omega\right)$, where $\varepsilon_{\infty}=9, \omega_{p}=1.3673 \cdot 10^{16} \mathrm{rad} / \mathrm{s}$ is the plasma frequency, $\gamma$ $=1 / \tau=1.0027 \cdot 10^{14} \mathrm{~s}^{-1}$ is the collision frequency and $\omega=2 \pi c / \lambda$. Later in the paper, an unrealistic value for the collision frequency of $\gamma=1 \cdot 10^{12} \mathrm{~s}^{-1}$ was used to provide better insight into the resonances.

\section{MODEL OF SLOW-PLASMON STANDING WAVE RESONANCES}

\section{A. Analytical model for surface plasmons in a straight finite length nanowire}

Throughout this paper, we modeled a cylindrical nanowire as a standing wave slow-SPP resonator. ${ }^{13-18}$ To calculate the expected frequencies at which the $m^{\text {th }}$ order resonance will occur, we used the standing wave condition: ${ }^{13}$

$$
\beta_{S P P} L_{t o t}=m \pi-\varphi
$$

where $\beta_{S P P}(\omega)=2 \pi / \lambda_{S P P}(\omega)=k_{0} n_{S P P}(\omega)=(\omega / c) n_{S P P}(\omega)$ is the propagation constant of the $\mathrm{SPP}, L_{t o t}$ is the total length of the nanowire, $m$ is an integer, and $\varphi$ is the phase shift due to reflection of the SPP mode at the wire ends. For simplicity we consider $\varphi=0$, which is equivalent to modeling the nanowire ends as perfect magnetic walls.

To obtain the resonance frequencies from the resonance condition, we need the dispersion relation $\beta_{S P P}(\omega)$ of the slow-SPP on a cylindrical nanowire. Such slow-SPP corresponds to the first order bound mode supported by the infinite cylinder, in which all fields show invariance with the angle in cylindrical coordinates $\theta$ (specifically this 
implies $n=0$ where the fields are multiplied by a harmonic term $\left.e^{i n \theta}\right)$. The dispersion relation of the slow-SPP mode is given by: ${ }^{10-12}$

$$
\begin{aligned}
& \frac{\gamma_{m} I_{1}\left(\gamma_{d} r\right) K_{0}\left(\gamma_{m} r\right)}{\gamma_{d} I_{0}\left(\gamma_{m} r\right) K_{1}\left(\gamma_{d} r\right)}=-\frac{\varepsilon_{d}}{\varepsilon_{m}} \\
& \gamma_{i}=\sqrt{\beta_{\text {slow-SPP }}^{2}-\varepsilon_{i} \mu_{0} \omega^{2}} \quad i=d, m
\end{aligned}
$$

where $\varepsilon_{d}$ is the permittivity of the surrounding dielectric, $\varepsilon_{m}$ is the metal permittivity following the Drude model and $r$ is the radius of the nanowire. The calculated real part of $\beta_{\text {slow-SPP }}(\omega)$ using the dispersion relation is shown in the inset of Fig. 2.

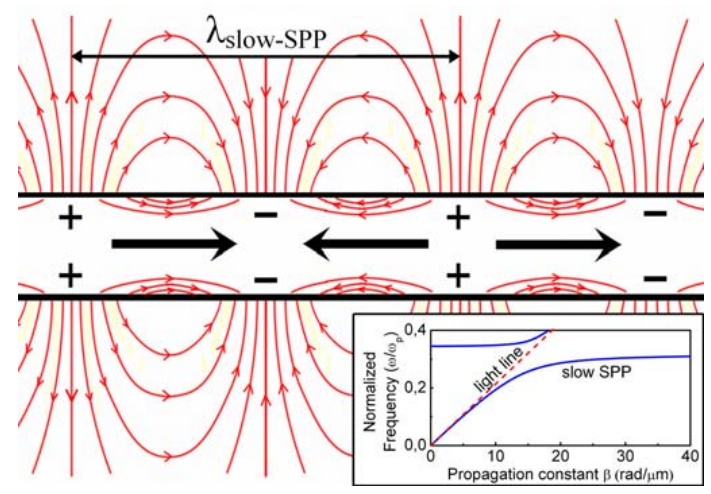

FIG. 2. Analytically calculated field at a certain instant for a slow-SPP standing wave resonance on an infinite cylindrical nanowire of radius $100 \mathrm{~nm}$. Red lines represent the electric field lines. The magnetic field (not shown) circles around the nanowire. Black lines represent the direction of current flow. The + and - signs represent the charge accumulation along the nanowire. Inset: Calculated dispersion relation of the slow-SPP mode.

Equations (1) and (2) yield the frequencies at which an incident excitation with the appropriate symmetry conditions can couple to the standing wave slow-SPP resonances. At these frequencies, dips in the transmission spectra will be seen. The inverse procedure can also be done: ${ }^{20}$ experimentally observing the extinction peaks in the spectra of transmission through arrays of nanofabricated finite length nanowires can be used to experimentally deduce the dispersion relation $\beta_{\text {slow-SPP }}(\omega)$.

The analytical field distribution ${ }^{12}$ of these slow-SPP standing waves is plotted in Fig. 2. The electric field is symmetrical with respect to the cylinder axis, and all fields show invariance with the angle in cylindrical coordinates $\theta$. If one looks at the electric field inside the metal nanowire, it can be seen that it consists of consecutive half- $\lambda_{S P P}$ sections with the longitudinal electric field component oriented towards alternating directions (in fact showing a sinusoidal variation with period $\lambda_{S P P}$ along the propagation direction). The current inside the metal nanowire will be proportional to the electric field and therefore will follow the same alternating pattern. 
To check the model, we simulated a plane wave incident into an array of gold nanowires, with the electric field parallel to the nanowire axis. The odd $m^{\text {th }}$ order slow-SPP standing wave resonances were clearly identified as extinction peaks in the transmission spectrum (the even $m^{\text {th }}$ order resonances were not observed since they cannot be excited by the incident electric field due to symmetry considerations). The field and current distributions showed strong resemblance to Fig. 2 except near the nanowire ends. The frequency of the first and second resonances showed a good agreement with the model, while the third resonance happened lower in frequency than expected. A similar shift was observed experimentally at high frequencies in Ref. 20 and it is suggested in Ref. 13 that this shift could be due to not considering a phase change $\varphi$ upon reflection at the ends.

\section{B. Resonator model for surface plasmons on a u-shaped nanowire}

To completely characterize the SPP resonances on a u-shaped nanowire, one should solve the full electrodynamic Maxwell equations on the particular geometry. However, it would be useful to have at our disposal a simpler model to predict the resonant frequencies and field distributions, as well as their dependence with geometrical parameters. It is reasonable to associate the resonances on a $\mathrm{u}$-shaped nanowire to the corresponding resonances on a straight nanowire of same total length as modeled above, although some inaccuracies arise from this process. The definition of the total length Ltot becomes ambiguous: we defined the total length of the nanowire as the length along its center, but the electric currents predominantly occur near the surfaces of the cylinder. Also the model does not take into account possible couplings between the propagating SPPs, especially at the bends of the ushaped nanowire, and it is known that SPP propagation along curved metal surfaces increases the attenuation and modifies the phase. ${ }^{21}$

\section{Current and field configurations yielding negative parameters}

Knowledge of the currents inside a finite length nanowire at each resonance (showing sinusoidal variation along its length) allowed us to predict the currents in the equivalent $u$ shaped nanowire. Just above the resonant frequency, these currents are known to lag behind the driving electromagnetic fields, thus creating either electric or magnetic dipolar moments which can be parallel and opposed to the incident electric or magnetic field. When the ushaped nanowires are sufficiently small compared to the wavelength, they can act as the unit cell of a homogenous metamaterial medium which then shows a Lorentz-type response in the effective permittivity or permeability respectively. If this response is strong enough it can result in negative constitutive parameters.

We simulated some examples of bent nanowire plasmon resonances leading to virtual current loops (VCL) and thus a Lorentz-type response on the magnetic permeability $\mu_{\text {eff }},{ }^{22}$ such as the first order resonance on a U-shaped nanowire (the well-known LC resonance discussed below) and the second order resonance on an S-shaped nanowire (not shown). 


\section{NUMERICAL RESULTS}

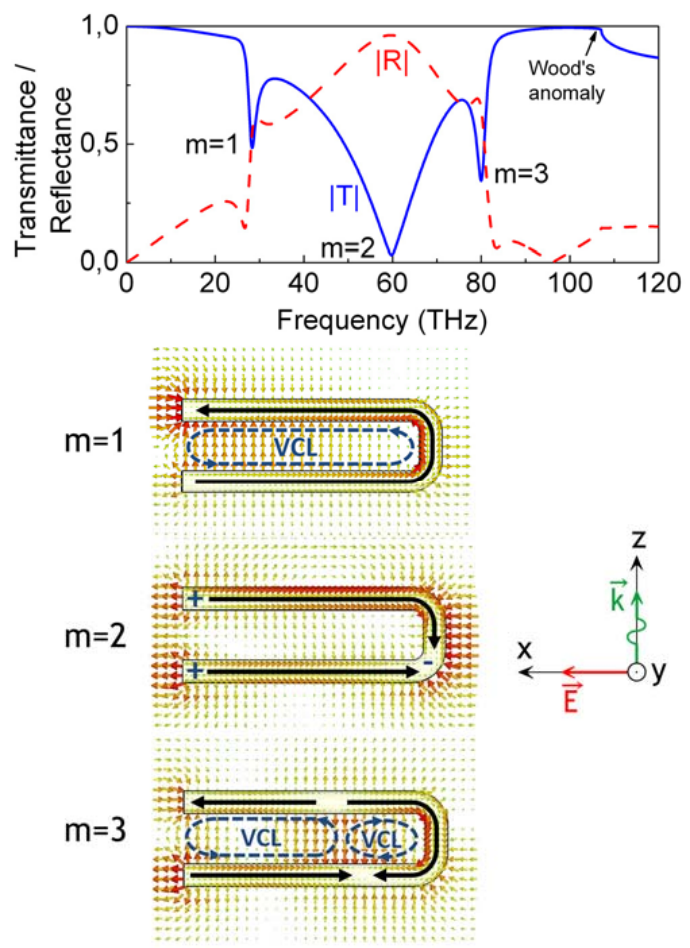

FIG. 3. Transmittance and reflectance spectra of the analyzed metamaterial upon plane wave excitation as shown in Fig. 1 . Geometrical parameters are: $r=100 \mathrm{~nm}, d=450 \mathrm{~nm}, L$ $=2125 \mathrm{~nm}, L_{\text {tot }}=4700 \mathrm{~nm}, a_{x}=a_{y}=2800 \mathrm{~nm}$. The three first resonances are seen as dips in the spectrum and the simulated electric field distribution is shown for a particular instant in each resonance. Black arrows indicate the current flow. Blue arrows indicate the VCLs (electric current loops closed by displacement fields).

\section{A. Resonances on a u-shaped nanowire}

We interpret the resonances that appear in a U-shaped nanowire at optical frequencies as slow-SPP standing wave resonances. Fig. 3 shows the simulated transmission and reflection spectra of a metamaterial composed of U-shaped nanowires (geometry as shown in Fig. 1), and the corresponding electric field and current distributions for each resonance. The geometrical dimensions used are $L=2125 \mathrm{~nm}, d=450 \mathrm{~nm}, r=100 \mathrm{~nm}$ and $a_{x}=a_{y}=2800 \mathrm{~nm}$. Results agree qualitatively with those in Ref. 8. As it can be seen, the current distributions in each mode labeled $m$ shows $m$ different sections of the nanowire with the current oriented at alternating directions. These current distributions agree with the known analytical currents shown in Fig. 2 for the slow-SPP standing wave resonances if the straight nanowire was bent into a u-shape. Also the electric and magnetic fields show the patterns that would intuitively arise from bending a finite straight nanowire. We believe that this field and current 
coincidence strongly supports the adequacy of the standing wave resonator model for the ushaped nanowire.

The first resonance is the so called LC-resonance of the SRR, and can be interpreted as the first plasmonic standing wave of the equivalent straight nanowire, (when $\lambda_{\text {slow-SPP }}=2 L_{\text {tot }}$ ). The virtual current loop (electric current closed by an electric displacement field) seen around all the structure and perpendicular to the incident magnetic field is responsible for a magnetic Lorentz-type response. On the other hand, the net electric dipolar moment shown by the charge distributions is small and oriented only in the $\mathrm{z}$ direction, so, as we will see, the electric response and the dipolar far field interaction between unit cells are weak on this first resonance.

The second resonance is usually called Mie resonance or electric plasmon resonance, and corresponds to the second plasmonic standing wave resonance of the equivalent nanowire (when $\lambda_{\text {slow-SPP }}=$ Ltot). The charge distribution shows a clear net electric dipole moment in the $\mathrm{x}$-direction (parallel to the incident electric field) and so this resonance shows a Lorentz-type electric response, which can yield a negative permittivity. Also, due to the net electric dipole moment, the position and strength of this resonance highly depends on the far-field dipolar interaction between unit cells.

The third resonance corresponds to the third plasmonic standing wave resonance of the equivalent nanowire. It can be seen that two opposing virtual current loops are created but one of them has a greater area and thus dominates, which allows the resonance to show a magnetic Lorentz-type response. It is this third resonance that we will use together with the second one to attain a negative refractive index. Its high frequency in comparison with lower order resonances makes it particularly sensible to a damping of the resonance due to a high electron mass inductance ${ }^{6}$ as compared to the geometrical inductance, so special care must be taken for this resonance to be strong enough as to show a negative real part of the permeability.

Above the third resonance, the well-known Wood anomaly appears where expected, ${ }^{23,24}$ corresponding to the frequency in which the first grating order changes from evanescent into propagating at grazing angle. The following higher order resonances occur above the Wood anomaly, where the first mode is propagating, and so are affected by great damping due to radiative losses.

We applied the effective parameter retrieval method ${ }^{25-27}$ using the simulated transmission and reflection spectra. The retrieved $\varepsilon_{\text {eff }}$ and $\mu_{\text {eff }}$ are shown in Fig. 4 and correspond only to propagation along $\mathrm{z}$ direction and for the polarization shown in Fig. 1. The homogeneous medium equivalence is poorly justified given that the unit cell's size in the propagation direction is only just below $\lambda / 4$ for the $3^{\text {rd }}$ resonance, however the results agree with expectations. The Lorentz-type resonances are clearly seen for $\mu_{\text {eff }}$ at the $1^{\text {st }}$ and $3^{\text {rd }}$ resonance, and for $\varepsilon_{\text {eff }}$ at the $2^{\text {nd }}$ resonance. The corresponding anti-resonance in the other parameter due to a finite wavevector ${ }^{28}$ can also be seen in each case.

In order to obtain a double-negative metamaterial, our aim was to push the second and third resonances closer, so that the negative permeability achieved by the $3^{\text {rd }}$ resonance occurred on the frequency range after the $2^{\text {nd }}$ resonance at which permittivity is seen to be negative. To 
achieve this we performed several simulations to deduce the dependence of the resonances with the geometrical parameters.

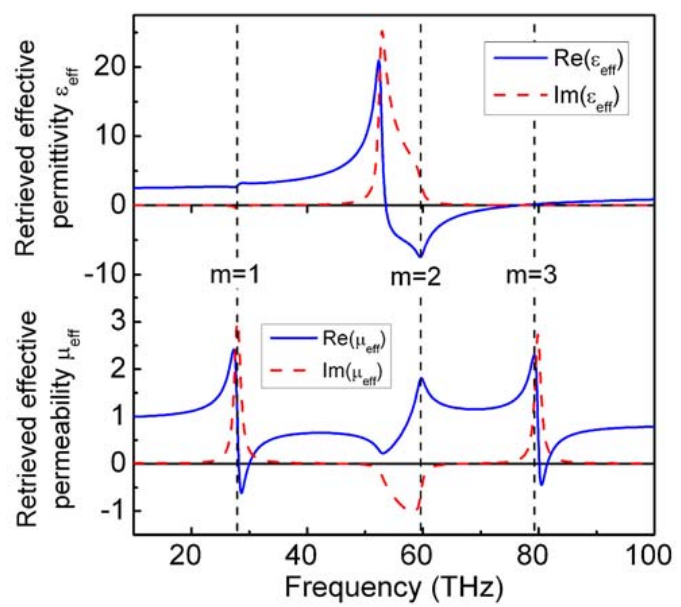

FIG. 4. Retrieved electromagnetic parameters from the simulated transmittance and reflectance spectra of Fig. 3. For the retrieval procedure, the equivalent metamaterial slab thickness was adjusted to the limits of the metal. The frequencies of the first three standing wave resonances are indicated with dashed lines.

\section{B. Resonance shifts through changes on geometrical parameters}

\section{Changes in the nanowire length}

We define the total length of the nanowire $L$ tot as the longitudinal length measured through its center, which is approximately given by $L_{t o t}=2 L+d$. By varying the total length we change the standing wave condition (1) on the propagation constant of the slow-plasmon mode, so shifts in the resonances occur as seen in Fig. 5(a). At relatively low frequencies (as compared to $\omega_{p}$ of gold) and a relatively large nanowire radius (compared with the penetration depth), the dispersion relation for the SR-SPP follows the light line very closely, so the expected resonance wavelengths accurately fall on $L$ tot $=n \lambda / 2$ (as in classical RF dipole antennas).

The third order resonance is always seen lower in frequency than the model prediction. This difference could arise from a frequency-dependent phase change $\varphi$ upon reflection of the slow-SPP at the nanowire ends, ${ }^{13}$ taken to be zero in our model.

\section{Changes in the nanowire radius (r)}

The dependence of the resonance frequency as a function of the nanowire radius is shown in Fig. 5(b). It is known that the SR-SPP dispersion relation $\beta_{\text {slow-SPP }}(\omega)$ is lowered in frequency when the radius of the nanowire is reduced, and the theoretical model predictions are seen to agree with simulations. Moreover, the resonances are substantially strengthened for 
increasing radius. This is due to a reduction in the propagation loss of the nanowire resonator due to the fields being less confined in the metal. This can be seen as a reduction in the imaginary part of $\beta_{\text {slow-SPP }}(\omega)$ with increasing radius determined by the dispersion relation in Eq. (2).
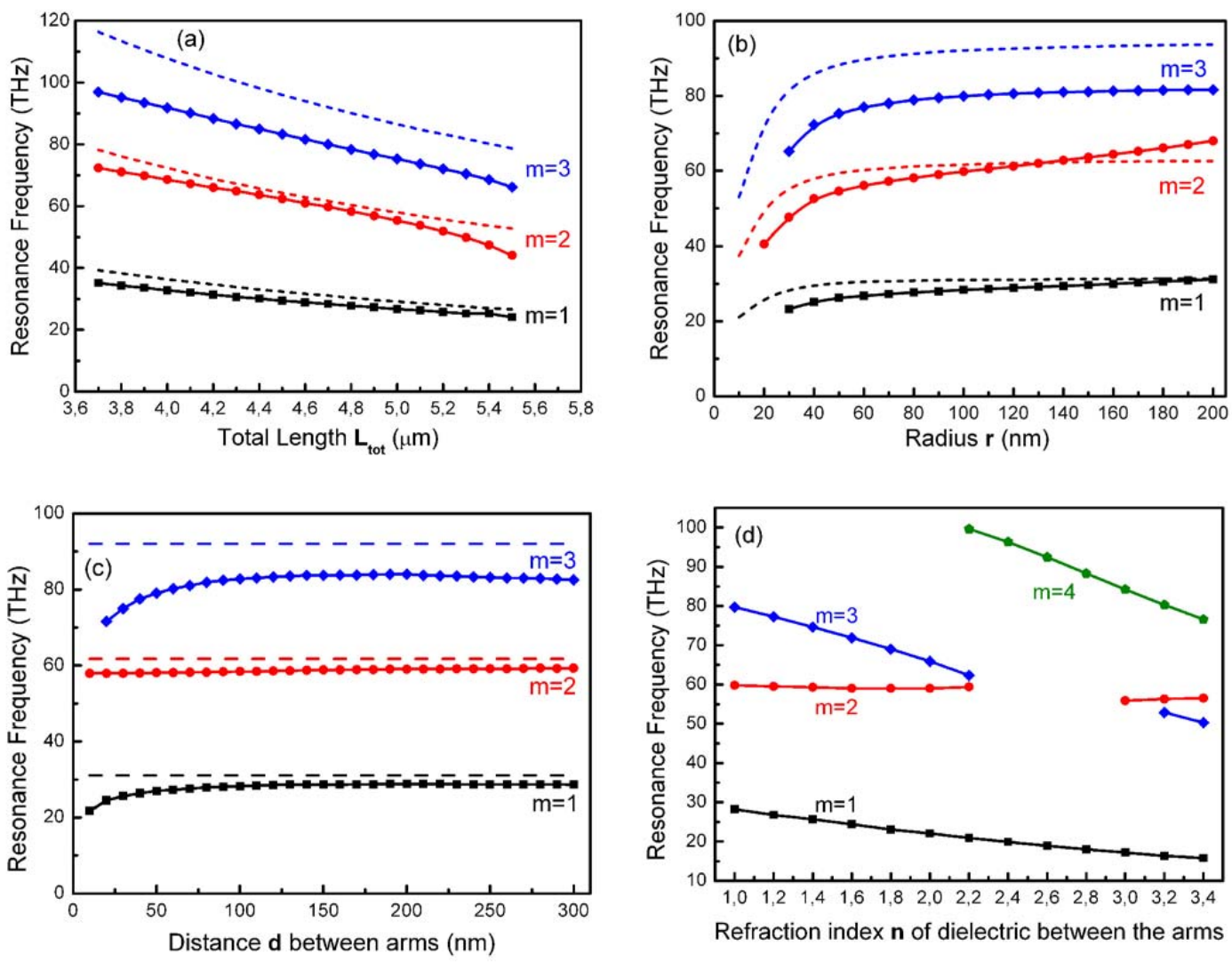

FIG. 5. Resonant frequency of the first standing wave modes of the metamaterial shown in Fig. 1 with varying (a) total length, (b) nanowire radius, (c) distance between arms, and (d) index of refraction of a dielectric inserted between the arms. The other geometrical parameters are kept constant to the values indicated in Fig. 3. The solid lines show the results from our simulations, while the dashed lines in (a) to (c) show the results calculated from the slow-SPP standing wave resonator model using equations (1) and (2). When two resonances cross in frequency and form a single dip, no line is plotted because the two resonances cannot be resolved.

\section{Changes in the distance between arms (d)}

In Fig. 5(c) we plot the resonant peak dependence with the separation between the two arms of the u-shaped nanowire. For a fair comparison, the total length of the nanowire Ltot was set constant, so we decreased the arm length $L$ as $d$ was increased. Our slow-SPP nanowire 
resonator model considers a straight nanowire and so cannot take into account distance between arms, so the model resonant frequencies in the graph are constant.

It can be seen that for small distances, the odd modes (which show opposite current flow on the two arms) shift down in frequency. We interpret this as a coupling effect: the electric field created by the SPP on one arm opposes the electric current flow of the SPP on the other, thus increasing the restoring force of the electron oscillations, which leads to a red-shift in the resonances. Also for reduced distances $d$, the area of the VCLs is reduced, which seriously weakens the effective magnetic response of the odd modes (in fact, for decreasing values of $d$ less than $20 \mathrm{~nm}$, the odd resonances are seen to gradually disappear).

The $2^{\text {nd }}$ mode is not affected by the strong red shift nor weakened, as both arms have the current flowing in the same direction and no increased restoring force occurs. In fact, when $d \rightarrow 0$ the second mode coincides with the first mode of a straight nanowire of length $L$ (instead of $L$ tot).

The coupling behavior of the odd modes agree qualitatively with an analytical model for the magnetic plasmon resonance (MPR) ${ }^{29}$ on two contiguous nanowires, which on Ref. 29 is studied on the same $1^{\text {st }}$ resonance of a $\mathrm{u}$-shaped nanowire which we study here. Unfortunately, applying the MPR formulae to plot the expected resonant frequency in this case was not suitable, as the condition $L \gg d \gg 2 r$ used in Ref. 29 does not hold in our structure. The MPR is accurate for very thin nanowires (thinner than a skin depth) where the slow-SPP mode is heavily confined inside the metal and slowed down, which enables a 'squeezed' resonance with a total length much smaller than the free-space wavelength. This small unit cell is advantageous for applying the homogeneous medium equivalence, with the payoff that at such thin nanowires great damping of the resonances occurs due to losses in the metal, as predicted by the dispersion relation in Eq. (2).

\section{Insertion of dielectric material between the arms}

By inserting a dielectric in the space between the two arms we found that the magnetic resonances ( $1^{\text {st }}$ and $3^{\text {rd }}$ mode), which have high field energy density in the space filled by the dielectric, shift down in frequency, while the electric resonance $\left(2^{\text {nd }}\right.$ mode $)$ which has low field energy density in the dielectric remains unchanged. The simulated results are shown on Fig. 5(d). The third resonance can be lowered in frequency towards the second resonance. As indicated later in this paper, this tuning of the third resonance can be used as a means of achieving a negative index of refraction.

\section{Changes in periodicity and far-field interactions}

Figure 6 shows the effect of varying $\mathrm{x}$ and $\mathrm{y}$ periodicity of the metamaterial on the SPP resonances. It is known that the periodicity of an array of nanoparticles can shift the SPP resonance frequency and behavior (lifetime and spectral width) both due to near-field coupling $^{30}$ (on nanoparticles nearly touching) and far-field coupling. Far-field coupling dominates for distances between nanoparticles greater than few tens of $\mathrm{nm}$, and was studied in full detail theoretically in Ref. 31 and measured experimentally in Ref. 32. 

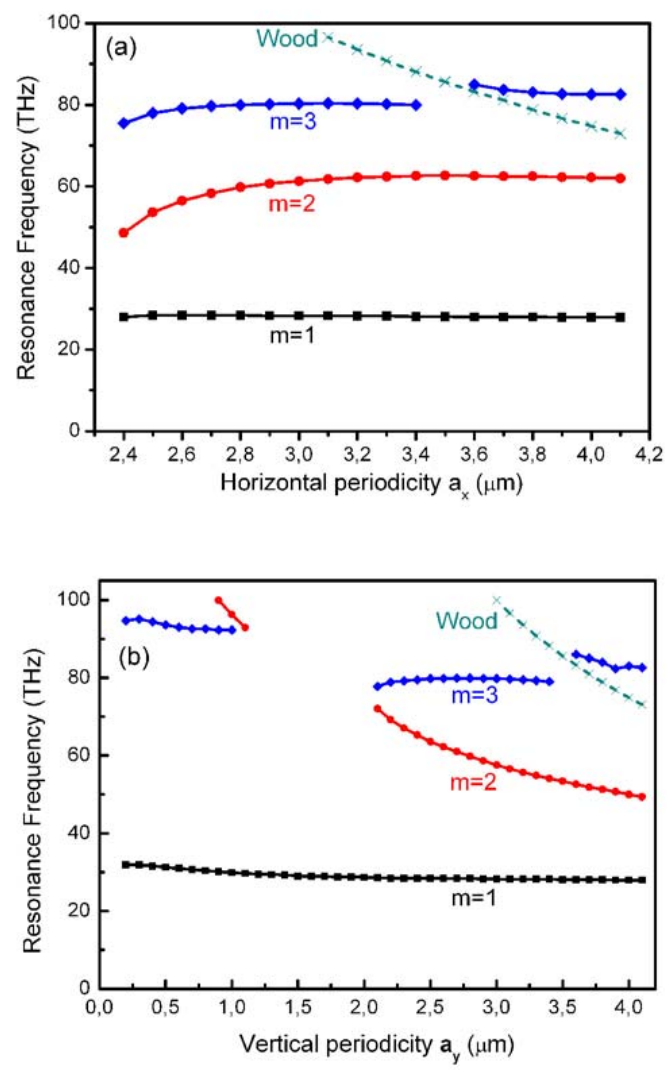

FIG. 6. Simulation results for the resonant frequency of the first three standing wave modes of the metamaterial shown in Fig. 1 with varying (a) horizontal (b) vertical periodicity. The frequency of the Wood anomaly is also shown. When two resonances cross each other in frequency and form a single dip, the graph does not show their spectral position as the individual resonances cannot be resolved.

For small periodicities $a_{x}$ with particles nearly touching on the $\mathrm{x}$ direction, the second resonance shifts down in frequency due to near field coupling (at the limit of touching nanoparticles, the metamaterial would resemble an array of infinite nanowires, which would behave as an effective plasma medium, ${ }^{33}$ where the second resonance would move down to zero frequency). For small vertical periodicities $a_{y}$ (with particles nearly touching in the $y$ direction), the second resonance strongly shifts up in frequency disappearing even above the third resonance, the reason for this is not clear. As the periodicity is increased, near-field coupling no longer dominates, and the effects of far-field coupling come into play. Far-field interaction of nanoparticles can be decomposed into two problems: the dynamic depolarization of a single particle and the dipolar interaction between point dipoles. ${ }^{31}$ In our case, only the second plasmon standing wave resonance shows strong net dipolar moment in the XY plane, in particular directed along the $x$-direction, so only this resonance shows significant dipolar interaction. This explains why the LC resonance frequency does not depend in periodicity, due to the lack of a net electric dipole moment. 



FIG. 7. Transmittance and reflectance spectra and retrieved electromagnetic parameters of the analyzed metamaterial upon plane wave excitation as shown in Fig. 1 when a dielectric with refractive index (a) 1.8 (b) 1.9 is introduced between the arms. Geometrical parameters are: $r=150 \mathrm{~nm}, d=450 \mathrm{~nm}, L=2125 \mathrm{~nm}, L$ tot $=4700 \mathrm{~nm}, a_{x}=a_{y}=2800 \mathrm{~nm}$. On the first case (a) a negative index of refraction is retrieved. On the second case (b), both resonances are too close together and disturb each other.

Figure 6 also shows the Wood anomaly. Theory predicts ${ }^{31}$ that the dipolar interactions for a given plasmon resonance exhibit strong variations when the periodicity allows a grating order to change from evanescent ( $a<a_{c}$, where $a_{c}$ is the critical grating constant) to radiative in character $\left(a>a_{c}\right)$. On Fig. 6(a) and 6(b) an example of such change occurs when the Wood anomaly crosses the third resonance. During this change, frequency-shifts in the plasmon resonance can be seen, and when the order starts to radiate, strong damping due to radiative losses affect the plasmon resonance, which is seen in the transmission spectrum (not shown) as a weaker dip with increased spectral width. Both effects were observed on simulations. 


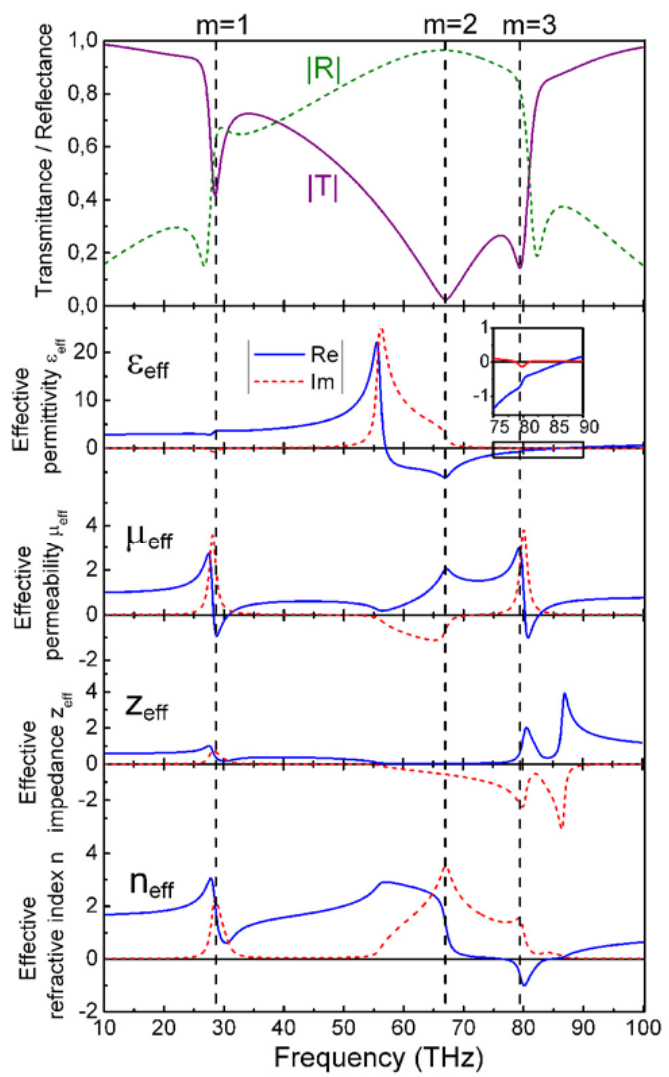

FIG. 8. Transmittance and reflectance spectra and retrieved electromagnetic parameters of the analyzed metamaterial upon plane wave excitation as shown in Fig. 1 with geometrical parameters $r=100 \mathrm{~nm}, d=450 \mathrm{~nm}, L=2125 \mathrm{~nm}, L$ tot $=4700 \mathrm{~nm}, a_{x}=2800 \mathrm{~nm}$, $a_{y}=2300 \mathrm{~nm}$. A negative index of refraction is retrieved.

\section{Negative index of refraction}

Considering only the metamaterial as seen in Fig. 1, without requiring additional wires or particles, we could achieve a simultaneous negative permittivity and permeability when the third resonance occurred just above the second one, and so obtain a LHM.

\section{Downshift of the third resonance through insertion of a dielectric between the arms}

Figure 7(a) shows the simulated transmission and reflection spectrum of the u-shaped nanowire with a dielectric of index $n=1.8$ inserted between both arms, and the retrieved effective parameters. The radius of the nanowire has been increased to achieve a stronger magnetic resonance. It can be seen that the $3^{\text {rd }}$ resonance has shifted down in frequency towards the $2^{\text {nd }}$ one, in agreement with Fig 5(d), therefore achieving a simultaneously negative $\operatorname{Re}\left(\varepsilon_{\text {eff }}\right)$ and $\operatorname{Re}\left(\mu_{\text {eff }}\right)$, and thus a negative $\operatorname{Re}\left(n_{\text {eff }}\right)$ at a frequency of $70 \mathrm{THz}$. 

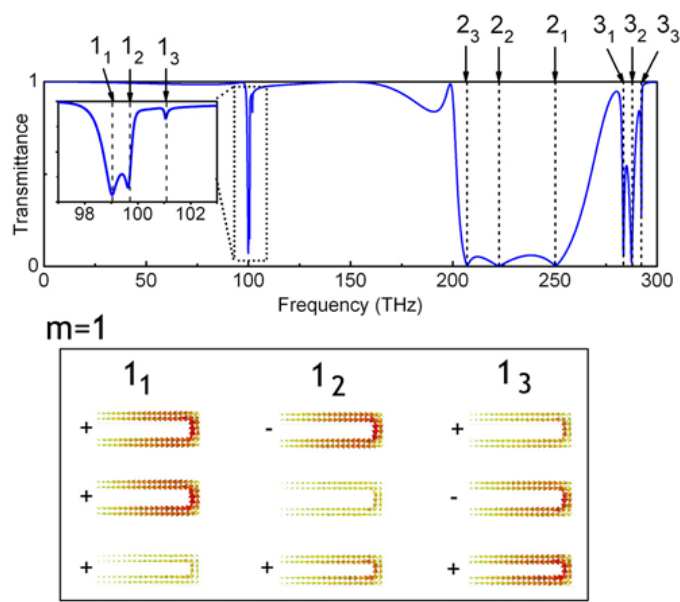

$m=2$

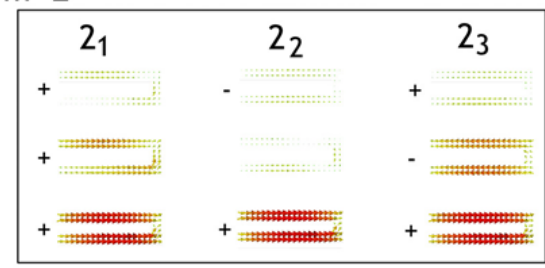

$m=3$

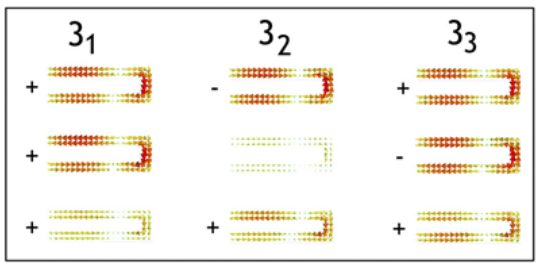

FIG. 9. Transmission and reflection spectra for three layers of metamaterial with geometrical parameters $L=375 \mathrm{~nm}, h=25 \mathrm{~nm}, w=40 \mathrm{~nm}, d=50 \mathrm{~nm}, a_{x}=a_{y}=600 \mathrm{~nm}, a_{z}=$ $250 \mathrm{~nm}$. For each resonance the current distributions on the three $\mathrm{u}$-shaped nanostrips of a unit cell as seen from above are depicted. The incident plane wave is incident upwards from the bottom in the representations. A plus or minus sign is added to each layer to indicate the phase of the electric current in that layer with respect to the first (the bottom) layer.

We noticed that if the third resonance comes too close to the second one, for a frequency separation below a certain threshold value, a drastic change in the phase of the transmittance (not shown) takes place, and the retrieved permeability and permittivity change from being both negative to being positive, but a peak where we had negative effective index is still observed due to the impedance approaching unity near the resonance. To achieve a negative index of refraction, we need the third resonance close to the second one, but not too close. As a rule of thumb we found that it is important that the two resonances show clearly distinguishable dips in the spectrum and do not merge to form a single dip. When the two 
dips are no longer distinguishable, no negative $\operatorname{Re}\left(n_{\text {eff }}\right)$ is retrieved. We believe that this is because when the two resonances coincide in frequency, the structure cannot support the two different field and current distributions simultaneously showing both electric and magnetic dipolar moments. We have found this criterion to be valid on all subsequent simulations. This is seen in Fig. 7(b), where a slightly higher dielectric than that on Fig. 7(a) was used: although a very slight variation in the absolute value of the transmittance spectra is seen, the two dips $m=2$ and $m=3$ merge into a single one, and drastic changes happen in the retrieved parameters resulting in a non-negative $\operatorname{Re}\left(n_{e f f}\right)$.

\section{Shift up of the second resonance by far-field dipolar coupling}

Figure 8 shows the simulated transmission and reflection spectrum of the u-shaped nanowire with an appropriately chosen $a_{y}$ periodicity [in agreement with Fig. 6(b)] that raises the frequency of the $2^{\text {nd }}$ mode closely below the $3^{\text {rd }}$ one (but not too close), and thus achieve a negative $\operatorname{Re}\left(n_{\text {eff }}\right)$ at a frequency of $80 \mathrm{THz}$.

\section{Stack of several layers of metamaterial}

We simulated the stacking of $N$ layers of the metamaterial with a period $a_{z}$ in the $z$ direction. We found that the peaks seen on the single layer which could be associated with the first, second and third slow-SPP standing wave resonances separate into $N$ peaks each. These $N$ peaks associated with each resonant mode are similar to the $N$ discrete normal modes supported by a nanoparticle chain. ${ }^{34}$ For simplicity in explanations we use the mode nomenclature $m_{n}$, where $m$ indicates the resonance order of the slow-SPP standing wave as used in a single layer, and $n=1 \ldots N$ indicates the order of the normal mode along the $z$ propagation direction, related with the relative phases of the mode at each layer. All the modes $m_{1 \ldots N}$ show the same $m$-mode resonance in each nanoparticle, but with different phases between the nanoparticles in each of the $N$ layers. Increasing the period $a_{z}$ causes the $m_{1 \ldots N}$ modes to move closer in frequency, and at $a_{z} \rightarrow \infty$ the modes become degenerate to form a single mode $m$, equal to that of a single layer. Also, if the number of layers is increased indefinitely $N \rightarrow \infty$, the modes $m_{1 \ldots N}$ would form a continuous spectrum showing the true unit-cell response of the infinite medium metamaterial.

To better study this complex set of resonances, we found that reducing the loss of the metal Drude model allowed a much easier identification of resonances when stacking the metamaterial, so on this last section we used in our simulations an unrealistically good collision frequency of $\gamma=1 \cdot 10^{12} \mathrm{~s}^{-1}$. This greatly reduced the resonance damping and also allowed us to very easily scale down the metamaterial to work in the near infrared. Also, to simplify the meshing during simulations, a rectangular nanostrip with height $h$ and width $w$ was used instead of a cylindrical nanowire of radius $r$. The slow-SPP mode fields and dispersion relations on such nanostrips can be calculated numerically, ${ }^{35}$ showing very similar behavior to a cylindrical nanowire.

The resonances seen on a stack of $N=3$ layers of the metamaterial are shown in Fig. 9 together with their electrical current distributions. It can be visually seen and confirmed with parameter retrieval that the new resonances $m_{1 . .3}$ can create new VCLs or new net electric dipoles which can give the resonances $m_{1 \ldots 3}$ either magnetic or electric Lorentz-type 
responses, respectively. For example, mode 23 clearly shows VCLs between nanoparticles and therefore shows a magnetic response.

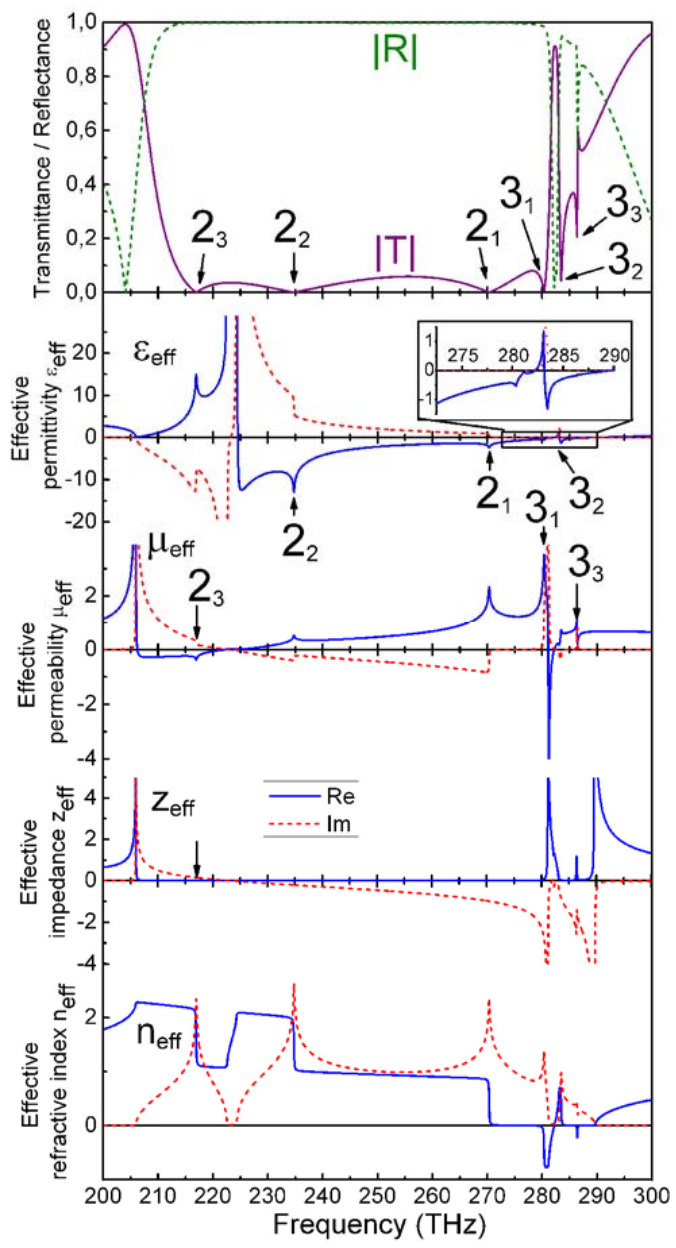

FIG. 10. Transmission and reflection spectra, together with retrieved electromagnetic parameters for three layers of the metamaterial with geometrical parameters $L=375 \mathrm{~nm}$, $h=25 \mathrm{~nm}, w=40 \mathrm{~nm}, d=35 \mathrm{~nm}, a_{x}=600 \mathrm{~nm}, a_{y}=500 \mathrm{~nm}, a_{z}=260 \mathrm{~nm}$. On resonance $3_{1}$, the permeability shows a Lorentz-type resonance yielding negative permeability and the effective permittivity is still negative due to the proximity of resonance 21 , therefore a negative index of refraction is retrieved around $280 \mathrm{THz}$.

Similar to the case with one layer, we adjusted the geometrical parameters of the $N=3$ layered metamaterial so that the electric resonance $2{ }_{1}$ (which is the highest in frequency of the $m=2$ resonances) occurred at a frequency just below the magnetic resonance $3_{1}$ (the lowest in frequency of the $m=3$ resonances), yielding a negative real part of the effective refraction index around $280 \mathrm{THz}$. Again, it was of utmost importance that both resonances 
where separated enough as to show distinct dips in the transmission spectrum so they were not interfering with each other. The transmission and reflection spectra and retrieved effective parameters are shown in Fig. 10.

Finally, the time-varying electromagnetic fields were simulated on the designed $N=3$ layered material at $282 \mathrm{THz}$, where $n_{\text {eff }}$ was found to be negative, and the backward wave propagation inside the LHM composed only of $u$-shaped nanowires was clearly observed, as shown in Fig. 11.
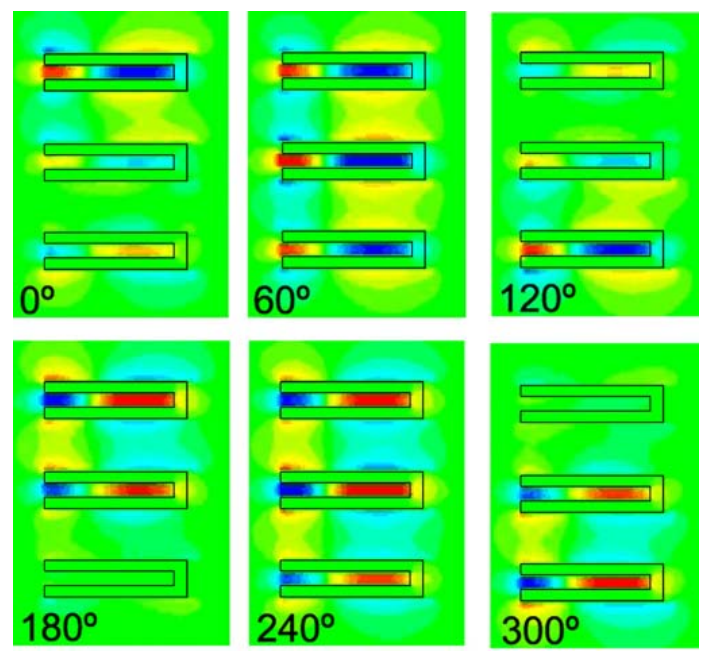

FIG. 11. Simulated magnetic field $\mathrm{H}_{\mathrm{y}}$ over time at $280 \mathrm{THz}$ for the $\mathrm{XZ}$ plane of a unit-cell of the metamaterial measured in Fig. 10. The incident plane wave propagates upwards from below. Backward phase propagation can be seen inside the metamaterial.

\section{CONCLUSION}

In summary, we have performed simulations confirming that the nanowire slow-SPP standing wave resonator model is very adequate to analyze the resonances in an array of SRRs, also called u-shaped or horseshoe-shaped nanowires. Use of the slow-SPP dispersion relation together with the standing wave condition can be useful to predict the frequency position and behavior of the several standing wave modes in the SRR as a function of geometrical parameters. To refine the predictions, far-field coupling between unit cells should be taken into account using the appropriate dipolar interaction model, ${ }^{31}$ and near field coupling between the two arms should also be taken into account when they are close together. Under certain restrictions (very thin nanowires and dimensions much shorter than the wavelength) the coupling between arms can be analyzed as the magnetic plasmon resonance (MPR) between two nanowires..$^{29}$

When the u-shaped nanowires were used as unit cell of a metamaterial, we saw through effective parameter retrieval methods how the various standing wave resonances can show either an electric or magnetic effective response depending on their current and field distributions, thus yielding negative constitutive parameters just above the resonant 
frequency. We demonstrated on simulations some methods of making the $3^{\text {rd }}$ resonance (which shows a magnetic response) exist in a frequency just above the $2^{\text {nd }}$ resonance (which shows an electric response) and so achieve a negative effective refractive index in the far infrared region of the spectrum. In contrast with typical metamaterials, negative permittivity and permeability have been obtained through different resonances on the same structure.

We also performed simulations on the effect of stacking the metamaterial into $N$ layers, and described how each standing wave resonance mode $m$ splits into $N$ normal mode resonances $m_{1 \ldots N}$ each showing its own electric or magnetic response. We performed simulations showing how these resonances can also be shifted adequately to achieve a LHM showing backward wave propagation.

\section{ACKNOWLEDGEMENTS}

Financial support by the Spanish MCyT and EU-FEDER under contract TEC2005-06923-C0303 is gratefully acknowledged. R.Ortuño and C. García-Meca also acknowledge financial support from the FPI grant program of Universidad Politécnica de Valencia.

\section{REFERENCES}

[1] V. G. Veselago, Sov. Phys. Usp. 10, 509 (1968)

[2] J. B. Pendry, Phys. Rev. Lett. 85, 3966 - 3969 (2000)

[3] R. A. Shelby, D. R. Smith and S. Schultz, Science 292, 77-79 (2001)

[4] T. J. Yen, W. J. Padilla, N. Fang, D. C. Vier, D. R. Smith, J. B. Pendry, D. N. Basov and X. Zhang, Science 303, 1494 - 1496 (2004)

[5] C. Enkrich, M. Wegener, S. Linden, S. Burger, L. Zschiedrich, F. Schmidt, J. F. Zhou, Th. Koschny, and C. M. Soukoulis, Phys. Rev. Lett. 95, 203901 (2005)

[6] J. Zhou, Th. Koschny, M. Kafesaki, E. N. Economou, J. B. Pendry and C. M. Soukoulis, Phys. Rev. Lett. 95, 223902 (2005)

[7] C. Rockstuhl, F. Lederer, C. Etrich, Th. Zentgraf, J. Kuhl, and H. Giessen, Optics Express 14, 8827-8836 (2006)

[8] C. Rockstuhl, T. Zentgraf, E. Pshenay-Severin, J. Petschulat, A. Chipouline, J. Kuhl, T. Pertsch, H. Giessen, and F. Lederer, Opt. Express 15, 8871-8883 (2007)

[9] R. H. Ritchie, Phys. Rev. 106, 874-881 (1957)

[10] C. A. Pfeiffer, E. N. Economou, and K. L. Ngai, Phys. Rev. B 10, 3038 - 3051 (1974)

[11] J. C. Ashley and L. C. Emerson, Surf Sci. 41, 615 (1974)

[12] J. Takahara, S. Yamagishi, H. Taki, A. Morimoto, and T. Kobayashi, Opt. Lett. 22, 475-477 (1997)

[13] T. Søndergaard and S. Bozhevolnyi, Phys. Rev. B 75, 073402 (2007)

[14] S. I. Bozhevolnyi and T. Søndergaard, Optics Express 15, 10869-10877 (2007)

[15] H. Ditlbacher, A. Hohenau, D. Wagner, U. Kreibig, M. Rogers, F. Hofer, F. R. Aussenegg, and J. R. Krenn, Phys. Rev. Lett. 95, 257403 (2005) 
[16] K. Payne, K. L. Shuford, S. Park, G. C. Schatz and C. A. Mirkin, J. Phys. Chem. 110, 21502154 (2006)

[17] T. Søndergaard and S. Bozhevolnyi, Optics Express 15, 4198-4204 (2007)

[18] G. Della Valle, T. Søndergaard and S. I. Bozhevolnyi, Optics Express 16, 6867-6876 (2008)

[19] V. A. Podolskiy, A.K. Sarychev, and V.M. Shalaev, J. Nonlinear Opt. Phys. Mater. 11, 65 (2002)

[20] G. Schider, J. R. Krenn, A. Hohenau, H. Ditlbacher, A. Leitner,F. R. Aussenegg, W. L. Schaich, I. Puscasu, B. Monacelli, and G. Boreman, Phys. Rev. B 68, 155427 (2003)

[21] Jiunn-Woei Liaw and Po-Tsang Wu, Optics Express 16, 4945-4951 (2008)

[22] J. B. Pendry , A. J. Holden, D. J. Robbins and W. J. Stewart, IEEE Trans. Microwave Theory Tech. 47, 2075-2084 (1999)

[23] R.W. Wood, Philos. Mag 4, 396-402 (1902)

[24] R.W. Wood, Phys. Rev. 48, 928-936 (1935)

[25] D. R. Smith, D. C. Vier, Th. Koschny, and C. M. Soukoulis, Phys. Rev. E 71, 036617 (2005)

[26] D. R. Smith, S. Schultz, P. Markos and C. M. Soukoulis, Phys. Rev. B 65, 195104 (2002)

[27] Xudong Chen, Tomasz M. Grzegorczyk, Bae-Ian Wu, Joe Pacheco, Jr., and Jin Au Kong, Phys. Rev. E 70, 016608 (2004)

[28] T. Koschny, P. Markos, D. R. Smith, and C.M. Soukoulis, Phys. Rev. E 68, 065602(R) (2003).

[29] A. K. Sarychev, G. Shvets and V. M. Shalaev, "Magnetic plasmon resonance," Phys. Rev. E 73, 036609 (2006)

[30] M. Quinten, A. Leitner, J. R. Krenn, and F. R. Aussenegg, Opt. Lett. 23, 1331-1333 (1998)

[31] M. Meier, A. Wokaun, and P. F. Liao, J. Opt. Soc. Am. B 2, 931- (1985)

[32] B. Lamprecht, G. Schider, R. T. Lechner, H. Ditlbacher, J. R. Krenn, A. Leitner, and F. R. Aussenegg, Phys. Rev. Lett. 84, 4721-4724 (2000)

[33] J. B. Pendry, A. J. Holden, W. J. Stewart and I. Youngs, Phys. Rev. Lett. 76, 4773-4776 (1996)

[34] W. H. Weber and G. W. Ford, Phys. Rev. B 70, 125429 (2004)

[35] P. Berini, Opt. Lett. 24, 1011-1013 (1999) 


\subsection{PAPER B}

$$
\text { J. Appl. Phys. 112, } 103104 \text { (2012) }
$$

\section{High order standing-wave plasmon resonances in silver $\mathrm{u}$-shaped nanowires}

Francisco J. Rodríguez-Fortuño, Rubén Ortuño, Carlos García-Meca, Javier Martí, and Alejandro Martínez 


\title{
High order standing-wave plasmon resonances in silver u-shaped nanowires
}

\author{
Francisco J. Rodríguez-Fortuño, Rubén Ortuño, Carlos García-Meca, Javier Martí and \\ Alejandro Martínez
}

Nanophotonics Technology Center, Universidad Politécnica de Valencia, 46022, Valencia, Spain

\begin{abstract}
Optical measurements of the transmission spectra through nanofabricated planar arrays of silver $u$-shaped nanowires on a silicon substrate resonating at infrared frequencies are performed. Good agreement with the numerically simulated surface plasmon standing wave resonances supported by the structures is found. Such resonances exhibit field enhancement and are able to provide magnetic and electric responses when used as the unit cell of a metamaterial. The magnetic excitation of the resonators using oblique incidence is shown to be drastically reduced by the existence of a high index substrate such as silicon.
\end{abstract}

\section{Introduction}

The optical properties of metallic nanostructures with features smaller than the wavelength of incoming light have proven useful in numerous applications. The study of their behavior involves the extremely interesting interaction of light with the plasma of free electrons in the metal. The variety of phenomena that arise from such coupling gives rise to the field of plasmonics [1]. Surface plasmons are waves of collective excitations of the conduction electrons in metals coupled to light and propagating through the surface of metallic conductors. Such waves are of special interest since the associated field mode profile is confined to the surface and decays exponentially away from it, allowing surface plasmons to break the diffraction limit of conventional dielectric waveguides [2], as well as achieving near field enhancement [3] and superlensing [4]. The possibility to tailor the nanostructure of metallic nanoparticles has enabled scientists to profoundly study and engineer such waves.

The interesting properties of such plasmons arise especially at and around the plasmonic resonances. Metallic nanoparticles show localized surface plasmon resonances (LSPR) in which strong collective electron oscillations are induced in the metallic nanostructures, with applications in spectroscopy and sensing [5] thanks to their field enhancement. These LSPRs are also responsible for strong electric and/or magnetic responses. The magnetic response in a collection of metallic particles arises when an incoming magnetic field excites plasmonic resonances such that they create an additional magnetic field, typically owing to the existence of closed loops of electric and/or displacement currents, called virtual current loops (VCLs) [6] . The magnetic response of such an ensemble is interesting in itself, since it is able to mimic, at optical frequencies, the magnetic response (characterized by a relative magnetic permeability $\mu_{r} \neq 1$ ) that exists in natural materials only at microwave and lower frequencies. A magnetic response is a necessary condition for many applications in the field of metamaterials, such as the famed invisibility cloak [7] and the perfect lens [8]. It could also be useful as an optical security signature [9]. 
In this paper we numerically calculate and experimentally observe, through far field measurements, the plasmonic resonances existing in an array of u-shaped resonators. This work follows previous theoretical studies about the use of this kind of resonator as a metamaterial unit cell for negative electric and magnetic responses [10].

(a)

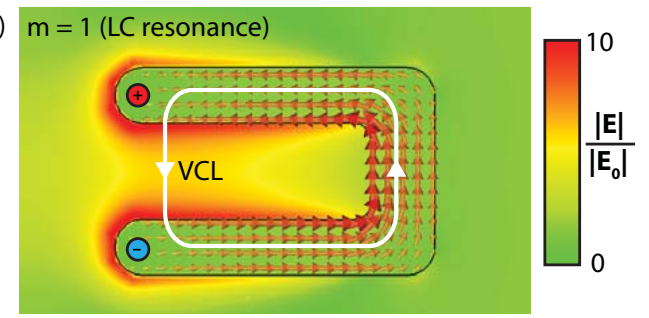

(b) $\mathrm{m}=2$ (electric resonance)

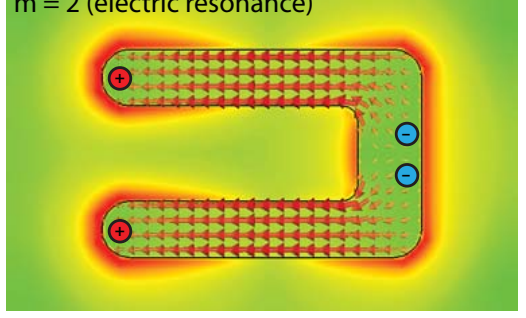

(c) $m=3$

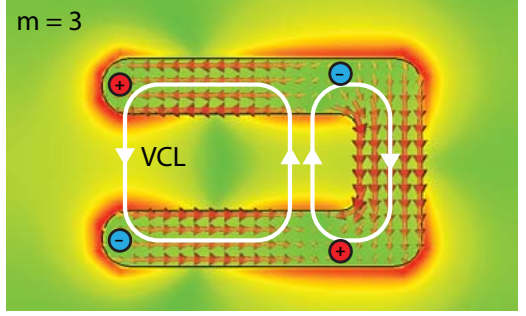

Fig 1. (Color online) Numerical simulation of the first three localized surface plasmon resonances in a unit cell of the $\mathrm{u}$-shaped particle array. Both the electric current density (red arrows) and the amplitude of electric field (normalized to the amplitude of the electric field in the same plane in absence of the metallic particle) are shown. An intuitive view of the virtual current loops (white loops) and the charge accumulations (+ and charge symbols) has been included.

\section{U-shaped resonator resonances}

Arrays of u-shaped metallic resonators, and similarly arrays of split-ring resonators (SRRs), are known to exhibit multiple plasmonic resonances [11-16]. The first resonance appearing at lower frequencies is the well known LC-resonance [Fig. 1(a)], in which a virtual current loop is formed along the whole structure, with electrons moving collectively throughout the full length of the metallic wire forming the particle, and with a strong electric field closing the loop between the two tips of the U shape. The VCL created by such a resonance is known to produce a magnetic field perpendicular to the plane of the resonator, so it can be used as unit cell in magnetic metamaterials [6]. The second resonance appearing is characterized by 
electrons moving back and forth along both legs of the particle in the same direction [Fig. 1(b)]. Such a resonance creates strong opposite charges at both ends of the particle, generating an electric dipole, which can be associated with an electric response. This resonance is sometimes called the electrical resonance. Further high-order resonances are observed, the first of which [Fig. 1(c)] shows an electric quadrupole and two VCL's (one of them dominating over the other) which also implies a magnetic response. As we proposed in a previous theoretical paper, this magnetic response can be combined with the electrical resonance to yield an effective negative index metamaterial [10]. In this paper we prefer the interpretation of all these resonances (LC, electric, and higher order resonances) as the plasmonic standing wave resonances of a nanowire [10,11,14].

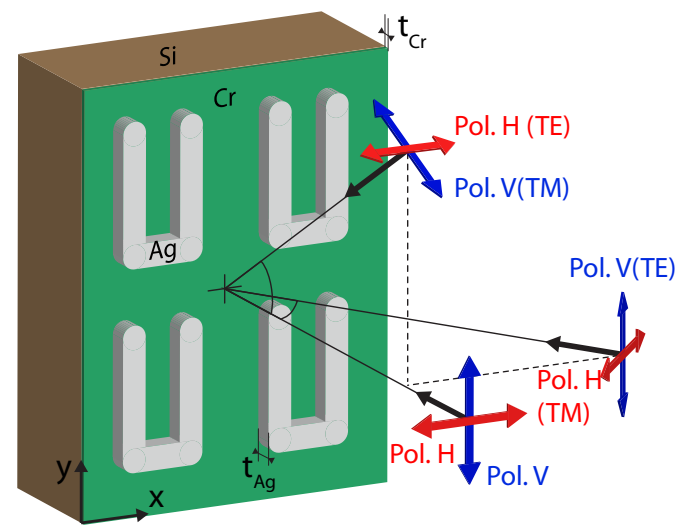

Fig 2. (Color online) Depiction of four unit cells of the sample showing the plane wave incidence for the normal and oblique cases.

It is known that metallic straight nanowires support bound propagating plasmon waves along their length [17]. One of these modes is the so-called slow, or short-range, surface plasmon, which is highly confined inside the metal. This mode is especially interesting in metallic nanowires because it is highly reflected at the ends of the nanowire (unlike other modes such as the long-range surface plasmon) and thus can form standing wave plasmon resonances along the length of the metallic nanowires $[18,19]$. A finite metallic nanowire of certain length then supports standing wave resonances with an integer number $m$ of halfwavelengths of the short-range surface plasmon. Therefore, this resonance shows $m$ sections of electric current in alternating directions along the length. One can intuitively picture the ushaped resonator as a simple bending of a straight nanowire, so that the LC resonance corresponds to the $m=1$ standing wave resonance and the electric resonance corresponds to the $m=2$ standing wave resonance. Thus the dispersion relation of slow plasmons in nanowires can be used to predict the position of the resonances in u-shaped nanowires [10], although the predictions are not exact due to various effects.

The resonances predicted with this model exist both in SRRs and in U-shaped resonators (which can be seen as a simplified version of the SRRs in which the gap is made wider) showing analogous field distributions. However, the specific resonant frequencies are slightly different for a SRR and a U-shaped nanowire with the same metallic wire length. A strong and highly confined field exists in the gap of the SRRs, so the geometry of the gap has 
strong effects in the spectral position of the first resonance. The LC model, taking into account the appropriate capacitance of the gap, is therefore required to determine its frequency [20], and careful refinements of the LC model can result in very accurate predictions for SRR resonant frequencies even in the optical region [21]. On the contrary, the field which closes the VCL in the first resonance of a u-shaped resonator is more evenly distributed across the length of the arms [Fig. 1(a)], and the resonant frequency does not depend strongly on small variations of the distance between them [10] -at least for the sizes under study here--, so modeling the resonances as slow plasmon standing waves along a straight nanowire of the same length is a convenient way to approximately predict and understand the frequencies of all the resonances $m=1,2,3, \ldots$ by using one same model, albeit lacking the high accuracy of specific LC models. In the case in which the gap is narrow, as happens on a SRR, the model becomes inaccurate to determine the spectral position of the resonances, but even in that case it is useful as a possible interpretation for the different resonance field profiles [14].

\section{Methods and materials}

Fabrication of silver u-shaped nanowires was performed on a silicon substrate by standard electron-beam-lithography. First, the clean silicon wafer was spin-coated with a $2 \mathrm{~nm}$ chromium layer to provide adhesion for the silver film. A layer of electron-sensitive resist was deposited upon the chromium layer. The sample pattern was then transferred to the electron resist via exposure to a focused electron beam. Subsequent chemical development removed the resist from all the regions exposed to the electron beam. A $20 \mathrm{~nm}$ silver metal film was then deposited on the sample by a sputtering process. Following deposition of the silver film, a chemical lift-off process removed the resist layer from the chromium surface. This left the periodic metallic u-shaped nanowires deposited directly on the chromium substrate through the openings in the resist mask. A schematic diagram of four unit cells of the resulting sample is depicted in Fig. 2. A scanning electron micrograph of the sample is shown in Fig. 3(a). The metallic u-shaped nanowires have the geometrical parameters indicated in Fig. 3(b). The infrared (IR) transmission spectra were obtained using a Bruker ${ }^{\mathrm{TM}}$ Fourier Transform IR spectrometer. The IR illumination was focused on the sample with a 0.4 numerical aperture $15 \mathrm{x}$ objective, using square apertures in the microscope beam path to adjust the illumination region to the whole sample area. Our measurements are mostly at frequencies below Wood's anomaly, so all the far field transmission below $53 \mathrm{THz}$ is associated directly to the zeroth order grating lobe exiting in a direction normal to the plane of the array into the substrate. The illumination was polarized using the polarizations indicated in Fig. 3(b).

The numerical simulations were performed using the commercial software CST Microwave Studio. The transmission spectra through an infinite array of $\mathrm{u}$-shaped nanowires were calculated by imposing periodic conditions at the boundaries of the unit cell. Both time domain and frequency domain simulations gave consistent results. The silicon substrate was modeled as a dielectric with refractive index $n=3.45$, and the silver $\mathrm{u}$-shaped nanowire was modeled using a Drude model $\varepsilon(\omega)=1-\omega_{p}^{2} /\left(\omega^{2}+i \omega \omega_{c}\right)$, with plasma frequency $\omega_{p}=1.37 \times 10^{16} \mathrm{rad} / \mathrm{s}$ and collision frequency $\omega_{c}=8.5 \times 10^{13} \mathrm{rad} / \mathrm{s}$. 


\section{Experimental results}

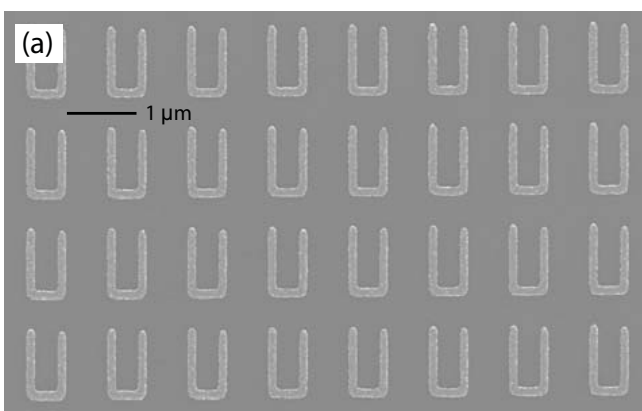

(b)
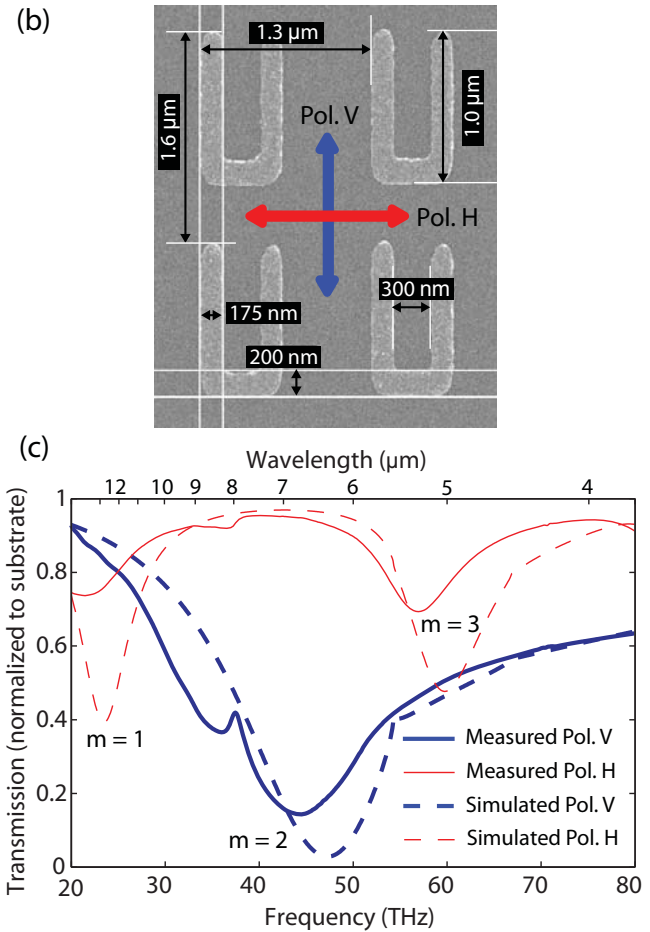

Fig 3. (Color online) (a) Scanning electron micrograph of a region of the fabricated sample. (b) Geometric sizes of the fabricated and simulated u-shaped resonators. (c) Optical measured and simulated transmission spectra for both horizontal and vertical electric field polarizations.

Figure 3(c) shows the measured transmission spectrum through the fabricated sample, normalized to the transmission through the substrate, together with the numerically simulated normalized transmission. Three distinct resonances can be clearly observed in the measurements and in fairly good agreement with the numerical calculations, corresponding to the first three standing wave plasmonic resonances in the u-shaped nanowire, $m=1,2,3$, whose electric current density and electric field strength are plotted in Fig. 1. We focus on 
these first three resonances. The position of these resonances as a function of geometrical parameters is discussed in detail in theoretical papers for $u$-shaped nanowires [10]. As can be seen, the periodicity of the u-shaped nanowire array is between 0.1 and 0.4 times the wavelength.

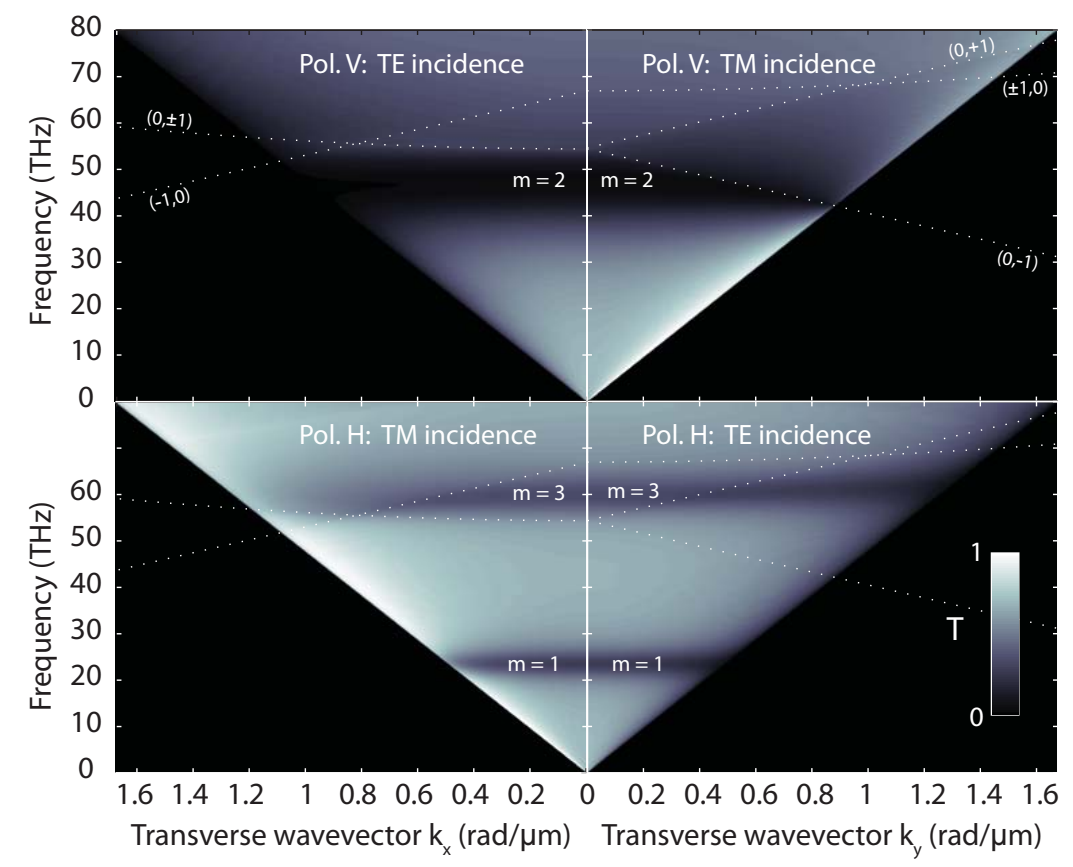

Fig 4. (Color online) Simulated power transmission spectra through the sample for oblique incidence (no normalization to the substrate was made). The power transmission for the two different TE and TM angles for each of the two different polarizations is shown. The angle of incidence was converted into the corresponding transverse wavevector. The plotted transmitted power corresponds only to the $(0,0)$ grating order. The frequencies at which higher grating orders arise at the output substrate are shown as white dotted lines.

Resonance $m=2$ is the electric dipole resonance, and can be excited with the electric field parallel to the u-shaped arms. According to symmetry considerations, this resonance can only be excited by the incident polarization labeled as Pol. V. The resonance is broad in the frequency domain because it couples easily to radiated plane waves, thereby showing high radiative losses and a low quality factor.

Resonances $m=1$ and $m=3$ can be excited at normal incidence by using an electric field oriented along the gap formed between the two ends of the U shape arms, and according to symmetry considerations, both resonances are only excited for the incident polarization labeled as Pol. H. These two resonances show VCL's and therefore exhibit a magnetic field in the direction perpendicular to the plane of the u-shape. In this case we are performing an electrical excitation of the magnetic resonances [6]. 
A noticeable kink is observed in the measurements around $8 \mu \mathrm{m}(38 \mathrm{THz})$. We do not know the origin of this sharp kink, and we did not manage to replicate it in the numerical simulations. Any effect of the surrounding environment and the measurement apparatus is taken care of when normalizing the measurement to the transmission through the substrate alone. A slight asymmetry in the arm length also cannot explain this kink. Alternatively, it could come from some material property of the silver or the chromium adhesion layer not taken into account in the simulation.

\section{Numerical results}

To further clarify the nature of these resonances, we performed additional numerical simulations. We computed the transmission spectra for oblique angles of incidence, as depicted in Fig. 2. The resulting transmission maps are shown in Fig. 4, where the numerically calculated power transmission through the sample is plotted as a function of frequency and transverse wavevector. The spectral position of the three resonances does not vary with the angle of incidence. This is a further indication that we are dealing with localized surface plasmon resonances in the individual nanoparticles, rather than propagating modes along the plane of the array. It is also an indication that the coupling between the nanoparticles of the array is relatively weak. The only instance of dispersive behavior in our results is seen at those frequencies at which higher order grating modes change from evanescent to propagating. These frequencies are usually associated with sudden changes in the transmission levels (Wood's anomaly) of the zeroth order mode and they depend on the angle of incidence according to the relation $2 \pi f_{\left(n_{x}, n_{y}\right)}=\left(c / n_{\text {subs }}\right)\left[\left(k_{x}^{i n c}+n_{x} 2 \pi / a_{x}\right)^{2}+\left(k_{y}^{i n c}+n_{y} 2 \pi / a_{y}\right)^{2}\right]^{1 / 2}$ where $\left(n_{x}, n_{y}\right)$ is the grating order, $n_{\text {subs }}$ is the refractive index of the substrate, $a_{x}$ and $a_{y}$ are the periodicities in the $x$ and $y$ directions, and $k_{x}$ inc and $k_{y}{ }^{i n c}$ are the transverse incident wavevectors. The frequencies for the first higher order modes are plotted as white dotted lines in Fig. 3 and their effect on the transmission spectra can be noticed.



Fig 5. (Color online) Normalized transmitted power for $\mathrm{V}$ polarization at normal incidence and at oblique 86 degree TE incidence, showing the magnetic excitation of the $\mathrm{m}=1$ resonance. Three different thicknesses of the silver $\mathrm{u}$-shaped nanowire are used: 30 $\mathrm{nm}$ (as in the fabricated samples), and higher thicknesses $150 \mathrm{~nm}$ and $300 \mathrm{~nm}$, to improve the magnetic excitation. 
In addition, we now turn our attention to the magnetic excitation of the $\mathrm{m}=1$ and $\mathrm{m}=3$ resonances, expected at high incident TE angles for V. polarization. Under those conditions, it is known that the incoming magnetic field has a component normal to the plane of the array and therefore can excite the LC $(m=1)$ resonance (the phase difference in the electric field arriving at both arms can be another source of coupling to this resonance, but can be neglected for highly subwavelength particles). This magnetic excitation is of special importance in many applications of SRRs and U-shaped nanowires. Figure 5 shows -in bluethe simulated transmission spectra for the TE incidence for V. polarization at two different incidence angles (corresponding to cross sections of Fig. 4). At normal incidence, the V. polarization can excite only the $m=2$ resonance, however under oblique incidence, the $m=1$ resonance can be very slightly excited by the incident magnetic field. Indeed, a very smooth shallow dip exists around $25 \mathrm{THz}$ for angles close to 90 degrees corresponding to such excitation, but it is very weak and is barely visible in Fig. 4 . This is actually consistent with previous experimental studies, where the magnetic excitation of resonances has been experimentally measured to be very small at oblique incidence in arrays of SRRs [6]. This is in contrast to the theoretical predictions of free standing U-shaped nanowires or SRRs where the resonances can be strongly excited by the magnetic field [10], so it is important to understand the reasons behind this reduced excitation.

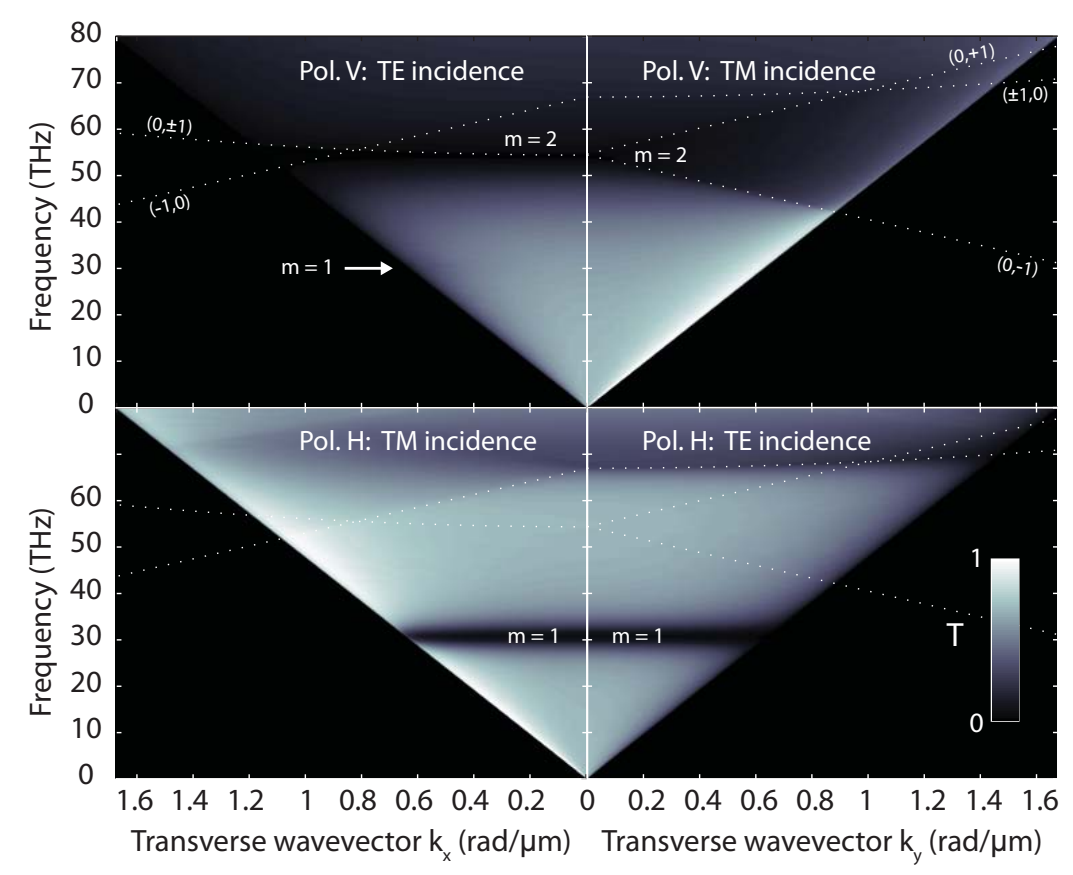

Fig 6. (Color online) Simulated power transmission spectra through the sample for oblique incidence, similarly to Fig. 4, for an increased thickness of silver of $150 \mathrm{~nm}$. The $\mathrm{m}=1$ resonance is stronger, and it can be slightly observed at high oblique angles for Pol. $\mathrm{V}$ at TE incidence. 


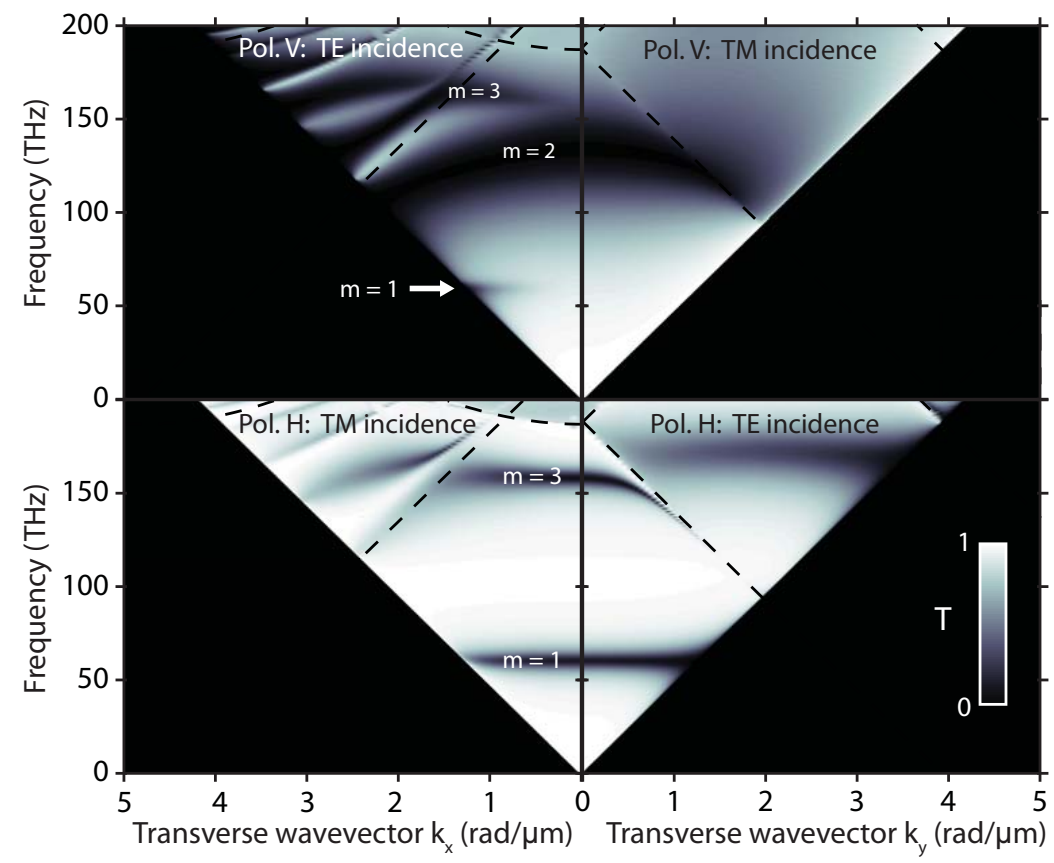

Fig 7. (Color online) Simulated power transmission spectra through the sample geometry for oblique incidence, similarly to Fig. 4 , but removing the substrate. The $\mathrm{m}=1$ resonance is clearly excited at high oblique angles for $\mathrm{V}$ polarization at TE incidence. The black dashed lines are the frequencies of Wood's anomalies.

A first attempt to increase this excitation is to make the $\mathrm{m}=1$ resonance stronger by increasing the thickness of the silver layer. Fig. 6 shows the transmission maps for a geometry with a silver thickness of $150 \mathrm{~nm}$. All resonances are slightly blueshifted, and clearly the $m=1$ resonance is stronger. The excitation of the $m=1$ resonance at TE incidence for V. polarization however still cannot be seen clearly in the figure, although it can be seen in the cross section shown in Fig 5. In addition Fig. 5 includes results for bigger thickness. We see that by increasing the metal thickness we can increase the magnetic coupling to the $\mathrm{m}$ $=1$ resonance at high angles of TE incidence in V. polarization, but we always obtain a weak excitation, even for metal thickness as big as $300 \mathrm{~nm}$, which is impractical.

A second attempt at increasing the magnetic excitation of resonances under oblique incidence is to use a substrate with a lower refractive index. We simulated the fabricated geometry (considering again a thin $30 \mathrm{~nm}$ layer of silver) but getting rid of the substrate (free standing particles), and we obtained the transmission map shown in Fig. 7. In this case, there is a blueshift in all resonances due to the big decrease in refractive index (notice the scale in the figure). This time, in the absence of substrate, the magnetic excitation of the modes under oblique TE incidence for $\mathrm{V}$. polarization at high angles is strong: the modes $\mathrm{m}=1,3$ (and even higher order odd modes) that are not seen at normal incidence, are clearly excited at oblique incidences. This is true even when using a very thin metal. This suggests that the presence of a high index substrate greatly decreases the efficiency of the magnetic excitation 
of resonances at oblique angles of incidence, and this should be taken into account when designing practical applications for such structures.

\section{Conclusions}

We have experimentally observed plasmon resonances on arrays of silver u-shaped nanowires. Our complete numerical modeling agrees well with the optical measurements, and shows that we are dealing with localized surface plasmon resonances associated with the slow-plasmon standing waves in the geometry, the first of which corresponds to the well known LC resonance. Owing to their plasmonic nature, the observed resonances present high field enhancement, with potential applications for spectroscopy and sensing. They also present electric and magnetic responses, with potential application for use as unit cell in metamaterials. The magnetic excitation of resonances by using oblique incidence is not strong in our geometry due the high index substrate, and to a lesser extent due to the small thickness of the silver layer. These results should apply also for SRRs in addition to the ushaped nanowires under study here, since both structures show similar field profiles and electric currents in their LC magnetic resonance.

\section{Acknowledgements}

We acknowledge financial support from the Spanish MICINN under contracts CONSOLIDER EMET CSD2008-00066 and TEC2011-28664-C02-02. F.J. Rodríguez-Fortuño acknowledges financial support from grant FPI of GV.

\section{References}

1. S. A. Maier, Plasmonics: Fundamentals and Applications (Springer, 2010).

2. W. L. Barnes, A. Dereux, and T. W. Ebbesen, "Surface plasmon subwavelength optics.," Nature 424, 824-30 (2003).

3. J. N. Anker, W. P. Hall, O. Lyandres, N. C. Shah, J. Zhao, and R. P. Van Duyne, "Biosensing with plasmonic nanosensors.," Nature materials 7, 442-453 (2008).

4. S. Kawata, Y. Inouye, and P. Verma, "Plasmonics for near-field nano-imaging and superlensing," Nature Photonics 3, 388-394 (2009).

5. K. A. Willets and R. P. V. Duyne, "Localized Surface Plasmon Resonance Spectroscopy and Sensing," Annual Review of Physical Chemistry 267-297 (2006).

6. C. Enkrich, M. Wegener, S. Linden, S. Burger, L. Zschiedrich, F. Schmidt, J. Zhou, T. Koschny, and C. Soukoulis, "Magnetic Metamaterials at Telecommunication and Visible Frequencies," Physical Review Letters 95, 5-8 (2005).

7. J. B. Pendry, D. Schurig, and D. R. Smith, "Controlling electromagnetic fields.," Science (New York, N.Y.) 312, 1780-2 (2006).

8. J. B. Pendry, "Negative Refraction Makes a Perfect Lens," Physical Review Letters 85, 3966-3969 (2000).

9. A. Martínez, C. García-Meca, R. Ortuño, F. J. Rodríguez-Fortuño, and J. Martí, "Metamaterials for optical security," Applied Physics Letters 94, 251106 (2009). 
10. F. Rodríguez-Fortuño, C. García-Meca, R. Ortuño, J. Martí, and A. Martínez, "Modeling high-order plasmon resonances of a U-shaped nanowire used to build a negative-index metamaterial," Physical Review B 79, 075103 (2009).

11. G. Boudarham, N. Feth, V. Myroshnychenko, S. Linden, J. García de Abajo, M. Wegener, and M. Kociak, "Spectral Imaging of Individual Split-Ring Resonators," Physical Review Letters 105, 255501 (2010).

12. N. P. Johnson, A. Z. Khokhar, H. M. H. Chong, R. M. De La Rue, and S. McMeekin, "Characterisation at infrared wavelengths of metamaterials formed by thin-film metallic split-ring resonator arrays on silicon," Electronics Letters 42, 1117 (2006).

13. C. Rockstuhl, T. Zentgraf, H. Guo, N. Liu, C. Etrich, I. Loa, K. Syassen, J. Kuhl, F. Lederer, and $\mathrm{H}$. Giessen, "Resonances of split-ring resonator metamaterials in the near infrared," Applied Physics B 84, 219-227 (2006).

14. C. Rockstuhl, F. Lederer, C. Etrich, T. Zentgraf, J. Kuhl, and H. Giessen, "On the reinterpretation of resonances in split-ring-resonators at normal incidence," Optics Express 14, 8827 (2006).

15. A. K. Sheridan, A. W. Clark, A. Glidle, J. M. Cooper, and D. R. S. Cumming, "Multiple plasmon resonances from gold nanostructures," Applied Physics Letters 90, 143105 (2007).

16. C.-Y. Chen, S.-C. Wu, and T.-J. Yen, "Experimental verification of standing-wave plasmonic resonances in split-ring resonators," Applied Physics Letters 93, 034110 (2008).

17. C. Pfeiffer, E. Economou, and K. Ngai, "Surface polaritons in a circularly cylindrical interface: Surface plasmons," Physical Review B 10, 3038-3051 (1974).

18. G. Schider, J. Krenn, A. Hohenau, H. Ditlbacher, A. Leitner, F. Aussenegg, W. Schaich, I. Puscasu, B. Monacelli, and G. Boreman, "Plasmon dispersion relation of Au and Ag nanowires," Physical Review B 68, 155427 (2003).

19. F. Neubrech, T. Kolb, R. Lovrincic, G. Fahsold, A. Pucci, J. Aizpurua, T. W. Cornelius, M. E. Toimil-Molares, R. Neumann, and S. Karim, "Resonances of individual metal nanowires in the infrared," Applied Physics Letters 89, 253104 (2006).

20. J. Zhou, T. Koschny, M. Kafesaki, E. N. Economou, J. B. Pendry, and C. M. Soukoulis, "Saturation of the Magnetic Response of Split-Ring Resonators at Optical Frequencies," Physical Review Letters 95, 223902-4 (2005).

21. V. Delgado, O. Sydoruk, E. Tatartschuk, R. Marqués, M. J. Freire, and L. Jelinek, "Analytical circuit model for split ring resonators in the far infrared and optical frequency range," Metamaterials 3, 57-62 (2009). 


\subsection{PAPER C}

Opt. Lett. 34, 3325-3327 (2009)

\section{Coaxial plasmonic waveguide array as a negative-index metamaterial}

Francisco J. Rodríguez-Fortuño, Carlos García-Meca, Rubén Ortuño, Javier Martí, and Alejandro Martínez 


\title{
Coaxial plasmonic waveguide array as negative index metamaterial
}

\author{
F. J. Rodríguez-Fortuño, C. García-Meca, R. Ortuño, J. Martí and A. Martínez \\ Valencia Nanophotonics Technology Center, Universidad Politécnica de Valencia, 46022, Valencia, Spain
}

\begin{abstract}
We propose the use of closely packed deep-subwavelength plasmonic coaxial waveguides which support backward propagating modes at visible frequencies, analogous to those in planar metal-insulator-metal geometries, as a negative index metamaterial. We show through simulation that the propagation properties of the metamaterial are determined by the dispersion relation of the constitutive waveguides. The metamaterial is polarization independent, is uniform in the propagation direction, and has subwavelength character in the transversal directions. Transmission loss through the structure is also analyzed.
\end{abstract}

Negative index metamaterials have lately attracted much interest from the scientific community due to their unique properties, particularly the possibility of attaining a perfect lens [1]. Such materials were first demonstrated at microwave frequencies, and continuous effort has led to realization of negative index metamaterials at optical wavelengths, most of which rely on metallic elements providing negative effective permittivity along with a resonant current loop leading to a negative effective permeability.

An alternative approach to achieve a negative index metamaterial is to use the negative group velocities present in certain metallic plasmonic waveguides to achieve negative refraction [2]. For example, on planar metal-insulator-metal (MIM) structures, surface plasmons propagating on the two interfaces can couple to each other if the metal films are close enough, and give rise to a backward mode in the dispersion relation [3]. This concept has been used to achieve planar (2D) negative refraction and superlensing of surface plasmons at optical and visible frequencies [4-6].



Fig. 1 (Color online) (a) Geometry of a plasmonic coaxial waveguide. (b) Proposed metamaterial: array of waveguides inside a metal. 


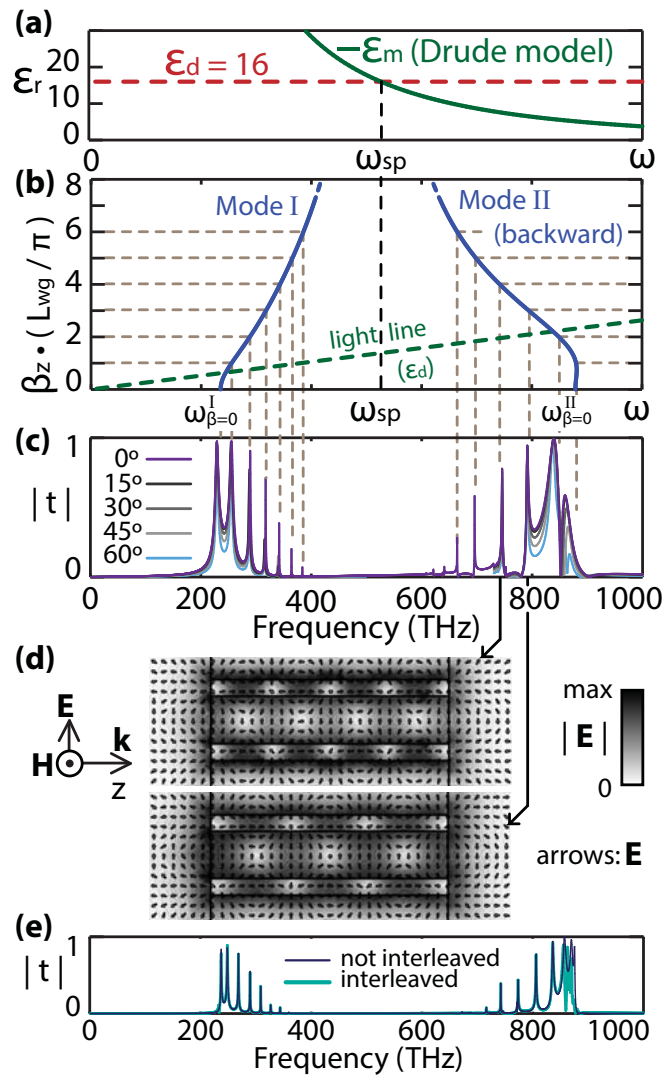

Fig. 2. (Color online) (a) Permittivity of metal $\left(\varepsilon_{m}(\omega)=1-\omega_{p}^{2} / \omega^{2}\right)$ and dielectric material. (b) Theoretical dispersion relation [10] for a single infinite coaxial plasmonic waveguide with dimensions $\mathrm{a}_{1}=10 \mathrm{~nm}$ and $\mathrm{d}=7 \mathrm{~nm}$ (modes $\mathrm{m}=1$ ). The light line is shown as reference. (c) Simulated field transmission spectra for different angles of plane wave incidence into a structure as depicted in Fig. $1(b)\left(D_{x}=D_{y}=70 \mathrm{~nm}\right.$ and $L_{w g}=100$ $\mathrm{nm})$ using low-loss metal. Dashed lines have been added at the expected waveguide resonances. (d) Simulated field distribution at two transmission peaks. (e) Simulated field transmission spectra for a structure with and without interleaved waveguides in the $\mathrm{x}$ and $y$ - directions (increased $D_{x}=D_{y}=L_{w g}=150 \mathrm{~nm}$ ).

A one-dimensional analogue [7] of the planar MIM waveguide is the coaxial plasmonic waveguide shown in Fig. 1(a) whose dispersion relation can be analytically studied [8-10] and on which backward modes have been reported [11]. For these modes, phase propagation occurs opposite to power flow. We propose the use of closely packed arrays of such coaxial plasmonic waveguides [Fig. 1(b)] with the aim that the resulting effective metamaterial shows the propagation properties of the constitutive waveguides [12] and therefore exhibits a negative effective index in the z-direction, independent of the polarization. 
The transversal confinement of the modes in plasmonic coaxial waveguides is evanescent, so waveguides with diameters smaller than the wavelength are possible [9]. This allows dense packing of the waveguides in the transversal dimensions, so the resulting metamaterial shows not only an infinitely subwavelength periodicity in the propagation dimension (since it is uniform) but also a subwavelength periodicity in the transversal dimensions, unlike other negative index metamaterials such as the so-called fishnet. This should avoid concerns over the validity of the homogenization.

Let us first study a particular example of a single infinite coaxial plasmonic waveguide with dimensions $a_{1}=10 \mathrm{~nm}$ and $d=7 \mathrm{~nm}$. Consider a metal modeled with a lossless Drude model using a plasma frequency of $\omega_{p}=1.367 \times 10^{16} \mathrm{rad} / \mathrm{s}$ and a dielectric material with refractive index $n=4$. The permittivity of both is shown in Fig. 2(a). Figure 2(b) shows the dispersion relation [10] for the modes in the waveguide which show one variation along the angular direction (that is, $m=1$, where the fields include a term $\exp [\operatorname{im} \varphi]$ ). We are interested in modes with $m=1$ because, unlike $m=0$ modes, they can be excited by a plane wave incident into the metamaterial.

At frequencies below $\omega_{s p}$, where $\varepsilon_{d}<-\varepsilon_{m}$, a positive-slope band labeled as I exists. This band has a cutoff frequency $\omega^{I} \beta=0$ due to the angular momentum imposed by the condition $m=1$, as described in Ref. [7]. The mode propagation constant increases with frequency and in fact diverges to infinity at the surface plasmon frequency $\omega_{s p}$, where the condition $\varepsilon_{d}=-\varepsilon_{m}$ is fulfilled. Above $\omega_{s p}$, we notice that $\varepsilon_{d}>-\varepsilon_{m}$. This condition, as described in theory [4], allows the appearance of a backward band (labeled as II). For the band to be backward, the cutoff

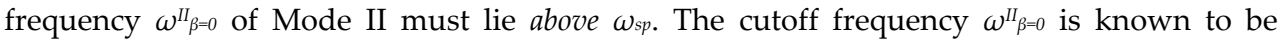
determined by the gap size $d$ between the metals [13], so the requirement $\omega^{I I} \beta=0>\omega_{s p}$ means that a very small distance $d$ (comparable to the plasmon decay length) is needed, which poses the major technological difficulty in the fabrication of the proposed negative index metamaterial. Notice that the proposed backward mode relies on plasmonic phenomena which occur only above $\omega_{s p}$. This allows us to work at visible frequencies, or rather obliges us to do so. If one wanted to have the negative mode at lower frequencies such as microwaves, a reduction in $\omega_{s p}$ would be needed, either by using a very high index dielectric or by reducing the plasma frequency of the metal, for example using low-frequency plasmons in thin-wire structures [14].

Once we have designed a waveguide supporting a backward mode at visible frequencies, we proceed to consider a structure consisting of arrays of several such waveguides, as shown in Fig. 1(b). Electromagnetic simulations using CST Microwave Studio were performed for such metamaterial with $D_{x}=D_{y}=70 \mathrm{~nm}$ and $L_{w g}=100 \mathrm{~nm}$. Notice the subwavelength periodicity in the transversal directions $(\lambda=350 \sim 500 \mathrm{~nm})$. The field transmission spectrum for a perpendicularly incident plane wave is shown in Fig. 2(c). Several transmission peaks associated to each of the two waveguide modes are observed. The peaks show high transmission, so this metamaterial is an example of negative extraordinary optical transmission [15]. As there is not perfect coupling between the plane waves and the waveguide mode, reflections will occur at the start and the end of the waveguides. This turns the waveguides into cavities showing standing wave resonances and is the origin of the transmission peaks under the condition $L_{w g} \approx n \lambda_{p} / 2$ where $n$ is an integer and $\lambda_{p}=2 \pi / \beta_{z}$ is the plasmon wavelength. This behavior is analogous to the Fabry-Perot resonances which would arise on a homogenous dielectric slab. The resonance condition in the waveguides is 
indicated with dashed lines in the dispersion relation, showing remarkable coincidence with the simulated metamaterial transmission peaks. The simulated electric field at two different peaks is shown in Fig. 2(d) showing the expected standing wave resonances (with plasmon wavelengths smaller for lower frequencies, as corresponds to a backward mode). All this suggests that indeed the effective metamaterial slab shows the propagation properties of the coaxial waveguides. The negative phase advance cannot be explicitly observed in this case since the fields show standing wave resonances.

Figure 2(c) also shows the tranmission spectra for plane waves incident at different off-axis angles. The position of the Fabry-Perot peaks are mantained, indicating that coupling of plane waves to the waveguide modes can happen at any incident angle. This is possible because there is no phase-matching condition required for such coupling.

As a note, we could think about extending this metamaterial for propagation of light in three dimensions, by interleaving coaxial plasmonic waveguides in the three directions of space. Thanks to the subwavelength diameter of the waveguides, the 3D unit cell would remain highly subwavelength. Also, the unit cell shows cubic symmetry. This could be a possible route for an isotropic negative index metamaterial at optical frequencies. Since the waveguide modes decay exponentially away from the waveguides, we expect that the interleaved waveguides along the $x$ and $y$ directions do not significantly alter the propagation along $z$ if the separation is big enough. The effect of the interleaved waveguides on the field transmission spectrum is shown in Fig. 2(e). In general, the mode shows little disturbance, except around $900 \mathrm{THz}$. The results are difficult to interpret, so we are working towards obtaining a full 3D dispersion relation of the structure (currently above our simulation capabilities).

Let us now analytically study the effect of losses in the proposed waveguides by considering a complex metal permittivity. The dispersion relation for a coaxial waveguide using the following permittivity for silver $\left[\varepsilon(\omega)=\varepsilon_{\infty}-\omega_{p}^{2} /\left(\omega^{2}-j \omega \gamma\right)\right.$ $\left.=3.7-\left(1.367 \times 10^{16} \mathrm{rad} / \mathrm{s}\right)^{2}\left(\omega^{2}-j \omega\left(8 \times 10^{13} \mathrm{~s}^{-1}\right)\right)\right]$ is shown in Fig. 3(a). The complex propagation constant $\alpha+j \beta$ accounts for the propagation loss. For mode II, the negative slope in $\beta$ and the negative sign in $\alpha$ both indicate that it is a backward mode, with power flow opposite to the phase advance [4]. If one repeats the transmission simulation as in Fig. 2(c) including losses, the spectrum shown in Fig. 3(b) is obtained. Resonance peaks broaden and transmission values decrease. The high attenuation reduces the standing wave ratio (the reflected waves will have much smaller amplitude) and therefore the negative phase advance inside the waveguide is now clearly observed [Fig. 3(c)], and the field distribution is almost identical to the analytical [10] backward mode of a single coaxial waveguide.

Losses are a big limitation in the maximum length of the proposed structure. In particular, the previously proposed 3D isotropic metamaterial would show propagation lengths comparable with the unit cell size, thus making the metamaterial useless in practice. Losses can be reduced by lowering the working frequency (e.g. microwaves) using materials with a lower surface plasmon frequency. The attenuation constant can also be reduced by using a bigger gap width $d$, in fact, high transmission through annular apertures has been demonstrated [16]. However, as we said earlier, a low $d$ is required to achieve a backward behavior, so a compromise should be sought. In the figures we have used $d=7 \mathrm{~nm}$, but further attempts at optimizing the geometrical parameters are desirable. This optimization 
should be eased by the fact that the metamaterial losses are characterized by the coaxial waveguide attenuation constant, which allows fast numerical [10] calculation or even approximate analytical expressions [7], unlike many metamaterial designs where losses must be retrieved from costly simulations. The introduction of plasmonic gain in the dielectric medium [17] would also be very interesting in order to attain a lossless negative index metamaterial in the visible spectrum.
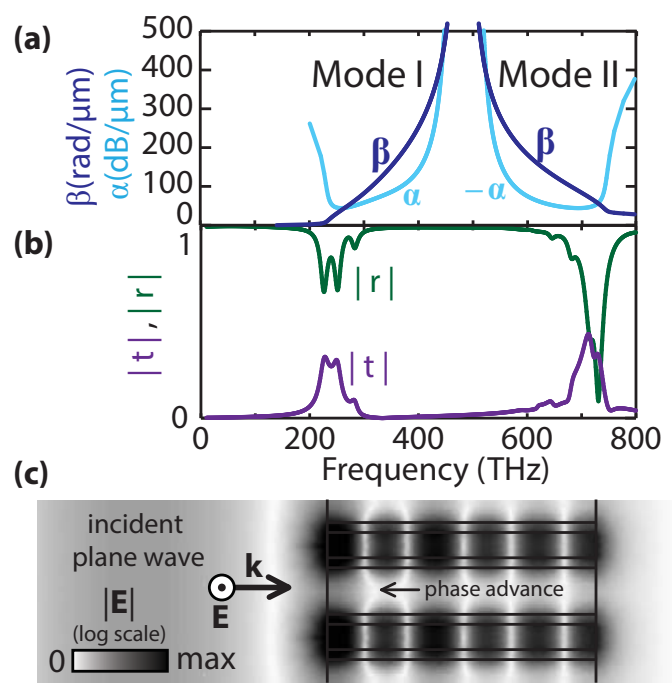

Fig. 3. (Color online) (a) Theoretical complex dispersion relation [10] of the coaxial plasmonic waveguide using realistic silver. (b) Simulated field spectra at normal incidence for a structure as depicted in Fig. 1(b) $\left(D_{x}=D_{y}=70 \mathrm{~nm}, L_{w g}=100 \mathrm{~nm}\right)$ using realistic silver. (c) Electric field at $700 \mathrm{THz}$ for the proposed structure (with $\mathrm{Lwg}_{\mathrm{wg}}=200 \mathrm{~nm}$ ).

In summary, we propose a metamaterial composed of arrays of deep-subwavelength coaxial plasmonic waveguides which support negative propagating modes. An extension to 3D is suggested. The metamaterial losses can be easily predicted through the waveguide dispersion relation, and pose a main drawback for the proposed geometry.

\section{Acknowledgements}

Financial support by the Spanish Ministerio de Ciencia e Innovación (MICINN) under contract CONSOLIDER (CSD2008-00066) is gratefully acknowledged. F. J. R.-F., C. G.-M., and R. O., also acknowledge financial support from grants of Generalitat Valenciana, MICINN and Universidad Politécnica de Valencia, respectively.

\section{References}

1. J. B. Pendry, "Negative refraction makes a perfect lens," Phys. Rev. Lett, 85, 3966-3969 (2000).

2. G. Shvets, "Photonic approach to making a material with a negative index of refraction," Phys. Rev. B 67, 035109 (2003). 
3. P. Tournois and V. Laude, "Negative group velocities in metal-film optical waveguides," Optics Communications 137, 41-45 (1997).

4. A. Alù and N. Engheta, "Optical nanotransmission lines: synthesis of planar left-handed metamaterials in the infrared and visible regimes," J. Opt. Soc. Am. B 23, 571-583 (2005).

5. H. Shin and S. Fan, "All-Angle Negative Refraction for Surface Plasmon Waves Using a MetalDielectric-Metal Structure," Phys. Rev. Lett. 96, 073907 (2006).

6. H. J. Lezec, J. A. Dionne and H. A. Atwater, "Negative Refraction at Visible Frequencies," Science 316, 430 (2007).

7. P. B. Catrysse and S. Fan, "Understanding the dispersion of coaxial plasmonic structures through a connection with the planar metal-insulator-metal geometry," Applied Physics Letters 94, 231111 (2009).

8. C. A. Pfeiffer, E. N. Economou and K. L. Ngai, "Surface polaritons in a circularly cylindrical interface: Surface plasmons," Phys. Rev. B 10, 3038 - 3051 (1974).

9. J. Takahara, S. Yamagishi, H. Taki, A. Morimoto and T. Kobayashi, "Guiding of a onedimensional optical beam with nanometer diameter," Opt. Lett. 22, 475-477 (1997).

10. The Matlab software that we developed for calculating the dispersion relation of coaxial plasmonic waveguides is available at http://www.consolidermetamateriales.es/tools/waveguide_3cyl.zip

11. K. Y. Kim, “Guided Dispersion Characteristics of Subwavelength Nanoscale Plasmonic Coaxial Lines," http://arxiv.org/abs/0905.0748.

12. J. B. Pendry, L. Martín-Moreno and F. J. Garcia-Vidal, "Mimicking Surface Plasmons with Structured Surfaces," Science 305, 847-848 (2004).

13. H. Shin, M. F. Yanik, S. Fan, R. Zia and M. L. Brongersma, "Omnidirectional resonance in a metal-dielectric-metal geometry," Applied Physics Letters 84, 4421-4423 (2004).

14. J. B. Pendry, A. J. Holden, D. J. Robbins and W. J. Stewart, "Low frequency plasmons in thinwire structures," J. Phys.: Condens. Matter 10, 4785-4809, (1998).

15. C. García-Meca, R. Ortuño, F. J. Rodríguez-Fortuño, J. Martí, and A. Martínez, "Negative refractive index metamaterials aided by extraordinary optical transmission," Optics Express 17, 6026-6031 (2009).

16. Y. Poujet, J. Salvi and F. I. Baida, "90\% Extraordinary optical transmission in the visible range through annular aperture metallic arrays," Optics Letters 32, 2942-2944 (2007).

17. M. A. Noginov, V. A. Podolskiy, G. Zhu, M. Mayy, M. Bahoura, J. A. Adegoke, B. A. Ritzo, K. Reynolds, "Compensation of loss in propagating surface plasmon polariton by gain in adjacent dielectric medium," Optics Express 16, 1385 (2008). 


\section{Chapter 3}

\section{Plasmonic analogues of quantum phenomena}

[...] There is a most remarkable coincidence: The equations for many different physical situations have exactly the same appearance. Of course, the symbols may be different-one letter is substituted for another-but the mathematical form of the equations is the same. This means that having studied one subject, we immediately have a great deal of direct and precise knowledge about the solutions of the equations of another. Introduction to Chapter 12-1, Vol. II.

THE SEARCH FOR ANALOGIES between different fields in physics is an interesting topic in itself. When two problems of very different physical nature share the same mathematical framework, the opportunity arises to experiment on one of the problems to learn about the other. In the case of plasmonics, lately there have appeared a series of analogies to quantum effects which enable exciting applications inspired by other disciplines of physics.

In this chapter we will describe in some detail the development and state of the art of two of the most well known plasmonic analogues of quantum phenomena: Fano resonances and Electromagnetically Induced Transparency. We will discuss the exciting applications in sensing and light stopping which arise from them. This will serve as introduction to our work on light trapping in split-ring-resonator-loaded photonic crystals [PAPER D]. Later, we also present our proposal of a plasmonic analogue of the quantum Hanle effect, to achieve polarization rotation in the presence of losses [PAPER E], with applications in switchable polarization control and sensing. Finally, a still unpublished electric metamaterial analogue to Meissner levitation will be outlined [Paper 19]. 


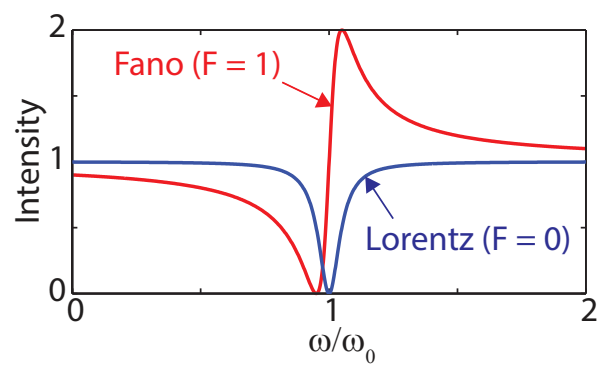

Figure 3.1: Fano lineshape following eq. (3.1) with $\gamma=0.05 \omega_{0}$ and $F=0$ or 1.

\subsection{Fano resonances}

Resonant phenomenona can be recognized throughout all the fields of physics, including mechanical, acoustic, electromagnetic and quantum theories, among many others. For many years, the spectrum of all resonances was believed to follow a Lorentzian lineshape, which stems from a simple differential equation. However, in 1935, H. Beutler observed asymmetric lineshapes in spectral atomic lines [151], which U. Fano managed to describe theoretically as the coherent interference of quantum amplitudes between different mechanisms of excitation [152]. In 1961, Fano summarized his theory with a complete and elaborate theoretical description for such resonances [153], bringing the concept of coherent interference of quantum wavefunction to mainstream physics. The spectral dependence of the so-called Fano resonance can be written as

$$
I \propto \frac{\left(F \gamma+\omega-\omega_{0}\right)^{2}}{\left(\omega-\omega_{0}\right)^{2}+\gamma^{2}},
$$

where $\omega_{0}$ is the position, $\gamma$ is the width, and $F$ is the so-called Fano parameter. Fig. 3.1 shows a Fano resonance lineshape, characterized by its sharpness and asymmetry. Fano resonances were believed to be a purely quantum mechanical phenomenon, and were studied in a myriad of quantum systems such as quantum dots and quantum nanowire systems $[154,155]$.

\section{Fano analogues}

It was later realized that Fano resonances could also occur in classical physics wherever wave interference phenomena exist $[156,157]$. Fano lineshapes take place when a discrete state interferes with a continuum of states. In fact, the first classical observation of such lineshape was Wood's anomaly, observed earlier, in 1902 [18] (see section 1.1 on the discovery of surface plasmons), which was traditionally associated to a singularity [158], but recently was pointed out to follow a Fano lineshape arising from the interference between surface waves in the grating and the incoming wave [159]. The simple model given by Fano therefore provides a general explanation of a universal 


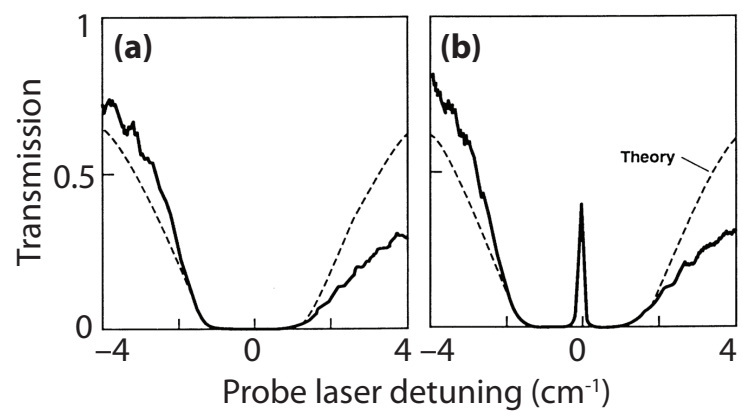

Figure 3.2: First experimental demonstration of EIT. Transmission spectra of a Strontium (Sr) sample (a) without and (b) with coupling laser, rendering the sample transparent in a narrow passband. Taken from [168].

phenomenon, which appears in various physical systems such as classical oscillators -e.g. coupled pendulums [160], - light-atom interactions [153], light interaction with structured matter, and atom-atom interaction (see review in Ref. [157]). Of particular interest to us are the observed Fano lineshapes in plasmonic structures and metamaterials [156], such as dolmen-shaped nanoparticles [161], non-concentric ringdisk cavities [162], metallic photonic crystals [163], asymmetrical split-ring resonators [164], and magnetic resonances in closely spaced nanoparticles [165].

Fano lineshapes, due to their dependence on a delicate interference scheme, provide a dramatic sensitivity to changes in geometry or material environment [156], with direct applications in chemical and biological sensing, detection of substrate deformation, or, if combined with non-linear media, for applications in electro-optics and switching.

\subsection{Electromagnetically Induced Transparency}

Electromagnetically Induced Transparency (EIT) is a remarkable quantum phenomenon: An opaque medium can be rendered transparent to a monochromatic probe laser light, due to the destructive quantum interference of the amplitude of the excited state of the atoms, arising from different excitation pathways: that from the probe laser and that from an additional coupling laser. The quantum amplitude of the excited state becomes zero, so that all atoms stay in their ground state, allowing the probe light through. In layman's terms, the electrons in the material are not allowed to move at a certain frequency, thanks to two opposite sinusoidal forces being applied to them [166, 167]. It was first experimentally realized in 1991 [168], and Fig. 3.2 shows the experimental results.

Because EIT depends on the precise quantum interference between two excitation pathways [169], the effect is highly frequency dependent. This results in a very narrow 

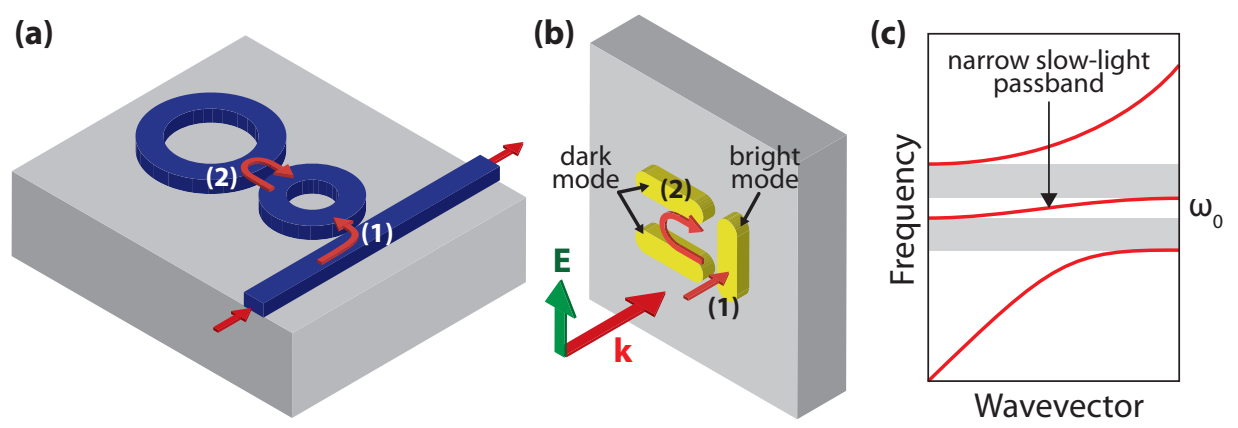

Figure 3.3: Classical analogues of EIT: (a) Coupled-Resonator Induced Transparency. (b) Unit cell of Plasmon-Induced Transparency. Labels (1) and (2) indicate the two interfering excitation pathways of the resonator. (c) Typical dispersion relation of an EIT, CRIT or PIT system.

transmission band [see Fig. 3.3(c)] with an extremely high dispersion in the refractive index $|\partial n / \partial \omega| \uparrow \uparrow$ associated with a slow group velocity of light $v_{g}=|\partial \omega / \partial k| \downarrow \downarrow$. The slow-light effect of EIT has been experimentally demonstrated to achieve an impressive reduction of the speed of light down to $17 \mathrm{~m} / \mathrm{s}$ in gas [170], or even bringing light to a complete stop in vapor [171] or solids [172].

\section{EIT analogues}

EIT is a coupled resonance phenomena, and therefore it can be interpreted as a Fano resonance in which the two coupled resonators have a sufficiently small frequency detuning and a strong contrast in their linewidths [156]. This means that other fields of physics can exhibit analogues of EIT. To achieve this, a certain so-called "bright" resonance, with a broad absorption spectrum, must be excited by two alternative pathways interfering destructively with each other, to prevent its excitation. After the first classical analog of EIT was discovered in magnetized plasma [173], many other classical analogs of EIT where found, including a simple model with masses and springs [174]. Of particular interest to us are the plasmonic and optical analogues of EIT.

- Coupled-Resonator Induced Transparency (CRIT): A phenomenon analogous to EIT was found to arise in ensembles of coupled classical LC oscillators [175]. This opened up the possibility of CRIT implemented with optical ring resonators placed next to an optical waveguide to achieve slow light propagation $[176,177]$ [see Fig. 3.3(a)]. Some of the proposals have been experimentally demonstrated $[178,179]$. The exhaustive comparative analysis by J. B. Khurgin [180] relates CRIT waveguides to the coupled-resonator optical waveguides (CROWs) proposed some years before [181]. 
- Plasmon-Induced Transparency (PIT): This plasmonic analog of EIT [182, 183] makes use of a bright (highly radiative and spectrally wide) dipolar plasmonic resonance of a nanoparticle, coupled to a nearby dark (non-radiative and narrow) plasmonic resonant nanoparticle. The bright mode can then be excited through two different pathways [numbered in Fig. 3.3(b)]:

1. Direct excitation from incident light: (incoming light $\rightarrow$ bright mode).

2. Indirect excitation from coupling to the nearby dark resonator: (incoming light $\rightarrow$ bright mode $\rightarrow$ dark mode $\rightarrow$ bright mode).

The interplay between both excitation pathways can be engineered such that destructive interference of the bright mode occurs, making its amplitude go to zero at the resonant frequency of the dark mode. A sharp resonance of nearly perfect transmission can arise in the broad absorption spectrum of the bright mode, in perfect analogy with quantum EIT. Magnetic resonances can also be used as the dark plasmonic mode [184].

CRIT and PIT both show the characterstic dispersion relation of EIT [shown in Fig. 3.3(c)] with high potential for slow-light and novel sensors. This potential is increased in the case of PIT due to the high field localization inherent to plasmonics.

\section{Stopping-light and time reversal inspired by EIT}

A common occurrence in the field of optical analogues is that the optical structures are sometimes rediscoveries of previously known phenomena. EIT analogues can be described as simple well-known coupled resonators. However, the uncovered analogy allows novel cross-disciplinary approaches.

In conventional slow-light optical waveguides, the achievable delay of optical pulses is fundamentally limited by the bandwidth of the pulse to be delayed. This theoretical limit is known as the delay-bandwidth product [185]. Coupled-resonator optical waveguides (like those analogue to EIT) where known to show slow-light propagation, but always limited by the delay-bandwidth product. However, as mentioned earlier, light was completely stopped using quantum EIT in a solid [172] for times greater than 1 second! This apparent violation of the delay-bandwidth limitation was only possible because a dynamic tuning was performed ${ }^{1}$. The same idea can thus be applied to the optical CRIT and PIT counterparts.

In a series of articles, M. Yanik and S. Fan proposed the stopping and storing of light beyond the delay-bandwidth product [186-188], and the time reversal of pulses [189], in all-optical EIT analogue devices. An extensive explanation of such dynamically tunable optical structures is provided in Ref. [190]. The mechanism behind light storing and reversal is the following:

\footnotetext{
${ }^{1}$ The delay-bandwidth product limitation assumes static devices.
} 

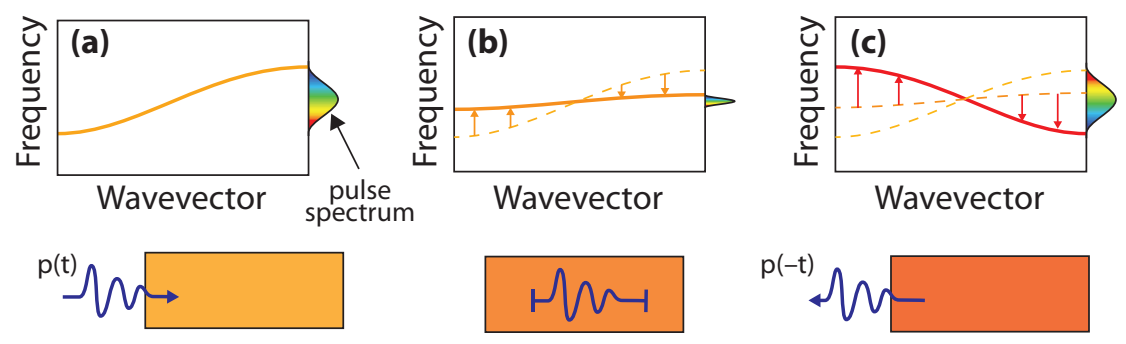

Figure 3.4: Dynamic tuning of the dispersion relation on the different steps of light stopping and time-reversal in EIT analogues

- A pulse of light enters a structure with a relatively flat band in its dispersion relation, typical of EIT and its analogues, as shown in Fig. 3.4(a).

- The structure is then dynamically varied so that the slope of the mode is reduced to zero. If the modulation is performed adiabatically ${ }^{2}$, the pulse frequency spectrum will coherently compress into a single frequency frozen pulse ${ }^{3}$, which remains coherently "stored" in the material [Fig. 3.4(b)].

- If the structure is further tuned to achieve a negative slope, as is possible in EIT structures, the spectrum of the signal is reversed and the group velocity is negative, so the pulse propagates backwards in the medium. The pulse arrives time-reversed at the input [Fig. 3.4(c)].

Such methods promise amazing capabilities that would potentially revolutionize the world of light processing. J. B. Khurgin realistically discusses the limitations due to losses and dispersion inherent to such structures in Ref. [192].

\section{Phase-coupled resonator EIT analogues}

In the previous proposals, the interfering resonators where directly coupled through spatial proximity. An alternative possibility is that the interfering resonators are not coupled through spatial proximity (direct coupling), but instead coupled by far field phase propagation (indirect coupling). This was proposed in Ref. [193], discussing the so called "indirectly" coupled resonator optical waveguides (named indirect CROWs, as opposed to conventional CROWs [181]). It was noted that if the distance between successive resonators was $\lambda / 2$ - where $\lambda$ is the wavelength in the waveguide,- then

\footnotetext{
2If the modulation is too fast (non-adiabatical) then the pulse frequency spectrum can "spill" to nearby bands. The problem is worse the closer the bands are.

${ }^{3}$ In conventional static geometries, different media can show different spatial wavevectors, but the temporal wavevector (i.e. frequency) always remains unchanged. However, in dynamic structures, changing the medium in time can lead to a change in the temporal frequency [191]. In the case of CRIT stopping of light, the spatial wavevectors remain unchanged, but the frequency spectrum is modified.
} 
a flat propagation band at the resonance frequency occurs inside the bandgap created by Bragg effects.

Although initially these structures were not related to EIT, it was later realized that their dynamic tuning could also allow light trapping and time reversal of pulses analogous to EIT. This was proposed in cascaded quantum-well resonators [194, 195] and later experimentally demonstrated [196]. The equivalent structure in plasmonics, named phase-coupled plasmon-induced transparency, was also proposed [197].

\subsection{Our work: SRRs inside a photonic crystal}

(a)

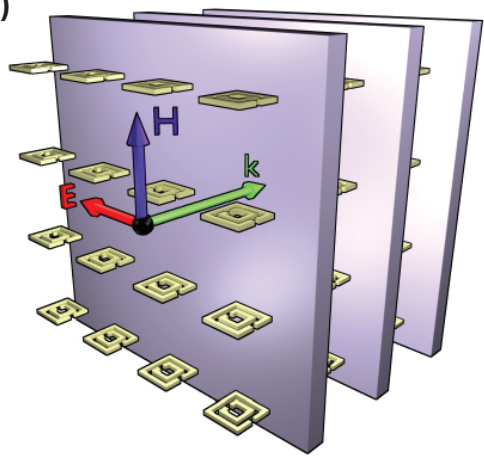

(b)
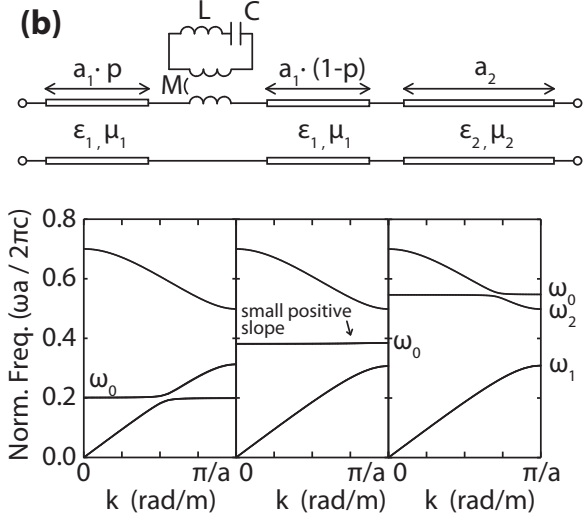

Figure 3.5: Split-ring resonators in a one-dimensional photonic crystal. (a) 3D depiction. (b) Model for the unit cell and dispersion relation.

As can be seen from the previous sections, the field of interfering resonators to achieve slow slight or even stopped light is clearly a fruitful field of study. Our contribution toward this field, described in [PAPER D], consists in the insertion of resonators -in this case SRRs, although any other kind of resonator would also work - in a one dimensional photonic crystal i.e. alternating layers of two dielectrics, as shown in Fig. 3.5(a).

Our motivation for studying such structure was not initially plasmonic analogues for slow light, instead we were searching for negative index effects. It was known that a rectangular microwave waveguide at cutoff shows an effective negative permittivity, which by itself prohibits propagation. Inserting SRRs resonating below the cutoff frequency, which also by themselves show an effective negative permeability which prohibit propagation, resulted in the combination $\mu<0$ and $\varepsilon<0$, providing a negative effective index which resulted in a backward propagation band [48, 198]. The geometry could also be mimicked in microstrip bandgap waveguides [199]. Inspired by such structures, we wanted to study the effect of inserting SRRs resonating within 
the bandgap of forbidden propagation due to the characteristic Bragg effects of a photonic crystal, looking for a negative index band.

What we found was that indeed a passband exists at the resonance frequency of the SRRs within the bandgap, but not necessarily backward. In fact, by tuning the parameters of the structure, the passband can be varied from a positive to a negative slope, including the intermediate case of a zero-bandwidth zero-slope band. The resulting dispersion relation resembles that of Fig. 3.4, so our structure has direct applications in light stopping and time reversal of pulses.

Our proposal is very similar to that of resonators placed at $\lambda / 2$ intervals described before, but in our case we have the added advantage that the desired flat band exists within a very broad bandgap created by the photonic crystal, so that the dynamic tuning of the flat band can be done faster, with a reduced leakage of the energy into nearby bands.

The periodicity of our structure is too large to explain the effects using effective medium theory, because Bragg effects clearly come into play, so in order to describe the behavior we used an equivalent circuit model for the unit cell, as shown in Fig. 3.5(b). In the equivalent circuit model, the SRRs where modeled as LC resonators coupled by a magnetic inductance $M$ [200], and the dielectrics where modeled as transmission lines, allowing phase propagation to take place. A transmission matrix relating the electric and magnetic fields at the output and input of the unit cell can be defined as [48]:

$$
\left(\begin{array}{c}
E_{\text {out }} \\
H_{\text {out }}
\end{array}\right)=\mathbf{T}\left(\begin{array}{l}
E_{\text {in }} \\
H_{\text {in }}
\end{array}\right),
$$

where the transmission matrix can be decomposed into the transmission matrix of each of the elements in Fig. 3.5(b):

$$
\mathbf{T}=\mathbf{T L}\left(\varepsilon_{1}, \mu_{1}, a_{1} p\right) \cdot \mathbf{S R R}(L, C, M) \cdot \mathbf{T L}\left(\varepsilon_{1}, \mu_{1}, a_{1}(1-p)\right) \cdot \mathbf{T L}\left(\varepsilon_{2}, \mu_{2}, a_{2}\right),
$$

where $\varepsilon_{i}, \mu_{i}$ are the two dielectric material parameters, $a_{1}$ and $a_{2}$ their thicknesses, $L$, $C$ and $M$ the effective parameters of the SRR, $p$ the relative position of the SRR within the air region, and $\operatorname{TL}(\varepsilon, \mu, a)$ and $\operatorname{SRR}(L, C, M)$ are the transmission matrices of a section of transmission line or a lumped element SRR (see section 2.6.1 in Ref. [48]) respectively, given as:

$$
\begin{aligned}
\operatorname{TL}(\varepsilon, \mu, a) & =\left(\begin{array}{cc}
\cos k a & i \eta \sin k a \\
(i / \eta) \sin k a & \cos k a
\end{array}\right), \quad k=\omega \sqrt{\varepsilon \mu}, \quad \eta=\sqrt{\mu / \varepsilon}, \\
\operatorname{SRR}(L, C, M) & =\left(\begin{array}{cc}
1 & \frac{-i \omega M^{2}}{L} \frac{1}{1-\left(\omega_{0}^{2} / \omega^{2}\right)} \\
0 & 1
\end{array}\right), \quad \omega_{0}=1 / \sqrt{L C} .
\end{aligned}
$$

Knowing the transfer matrix of a unit cell, the dispersion relation of the periodic structure can be obtained by equating the sum of the diagonal elements of the transfer matrix $\mathbf{T}$ to $2 \cos (k a), k$ being the desired mode wavenumber $[48,52]$. This gives rise to all the results detailed in [PAPER D], including the analysis of loss and dispersion, confirmed by numerical electromagnetic simulations. 


\subsection{Our work: Polarization conversion in plasmonic analogue of Hanle effect}

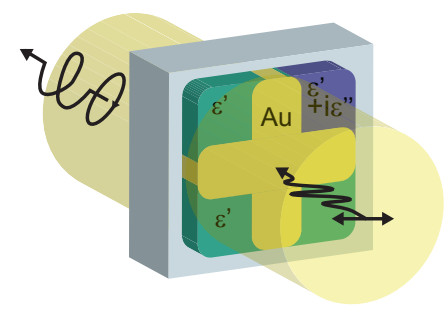

Figure 3.6: Polarization conversion in a plasmonic nanocross with non-uniform losses in the surrounding dielectric.

In the attached [PAPER E] we present the following contributions:

- We develop a formalism for the coupling between eigenmodes in lossy structures. Any electromagnetic structure, such as a plasmonic particle, exhibits resonant modes - associated to eigenmodes of the electromagnetic equationwhich, if the materials are lossless, form an orthogonal set, i.e. the modes are uncoupled. In our work, we use a non-hermitian formalism ${ }^{4}$ to describe the lossmediated coupling that exists between the eigenmodes of any electromagnetic structure with a permittivity that includes loss $\varepsilon(\mathbf{r})=\varepsilon^{\prime}+i \varepsilon^{\prime \prime}$. A non-hermitian formulation of quantum mechanics [201, 202] can be applied to systems in which the Hamiltonian does not show hermicity, and is specially useful to describe dissipative systems. Such formulation has been succesfully employed to describe higher harmonic generation [203] and unidirectional mode coupling in waveguides [204]. In the paper, we find the coupling between plasmonic modes to be proportional to the overlap integral of the fields of the uncoupled modes in the lossless structure, weighed by the local loss $\varepsilon^{\prime \prime}$ (see eqs. (3) and (4) in [PAPER E]). This means that a non-uniform loss in the particle can be used to induce coupling between resonances. The formalism could be useful in the study of lossy plasmonic structures and metamaterials.

- We show polarization conversion due to loss coupling in a nanocross plasmonic particle. The nanocross under study (see Fig. 3.6) has two dipolar plasmonic resonances: vertical $|V\rangle$ and horizontal $|H\rangle$, which are degenerate if no non-uniform losses are induced. Incident vertical and horizontal linearly polarized light couples only to the vertical and horizontal resonances, respectively. If a non-uniform loss is introduced (as we do by increasing the imaginary

\footnotetext{
${ }^{4}$ The presence of losses in our system causes the operator of the electromagnetic master equations to be non-hermitian.
} 
part of the permittivity on a quadrant of the surrounding dielectric) then the $|H\rangle$ and $|V\rangle$ resonances become coupled, in accordance to the previously described formulation, transferring energy from one to the other. This means that even if an incident horizontal polarization excites only the horizontal resonance, there will be a transfer of energy into the vertical resonance, so that the scattered light will show a combination of both horizontal and vertical polarizations. This polarization conversion is stronger the higher the induced losses are (see Fig. 3 in [PAPER E]). We achieve high conversion in a structure of highly subwavelength thickness. In addition, losses induced by external stimuli — such as thermal, electrical or optical signals - could be used to control the polarization conversion. The effect could also be used for the sensing or measurement of lossy substances, since it provides us with a measure of the degree of loss in a given region.

- We present an analogy between the loss-coupling polarization conversion and the quantum Hanle effect. The quantum Hanle effect [205] describes the polarization rotation experienced by light as it interacts with an atomic medium in the presence of a magnetic field. The effect is so sensitive that it is used in high resolution atom spectroscopy [206] and the detection of magnetic fields in stars [207, 208]. The Hanle effect is shown in Fig. 1(a-b) of [PAPER E]. The p-orbitals of an atom are excited by the two circular polarizations - left and right-handed, - and are degenerate in the absence of a magnetic field. An incoming linearly polarized light, being a superposition of left- and right-handed circular polarizations, will excite both states, which due to their degeneracy will decay in phase, preserving the polarization of scattered light. However, if a magnetic field is applied, the electronic states become coupled, and their energy levels split. Now, they decay with slightly dissimilar time constants, adding a phase difference to both polarizations, so that the scattered light will be elliptically polarized.

In the Hanle effect, the magnetic field is responsible for the coupling between the two resonances, causing polarization conversion. A stronger magnetic field results in a stronger coupling and increased conversion. In analogy, our plasmonic resonances are coupled by the non-uniform losses, causing a polarization conversion. Higher losses result in stronger coupling and a higher polarization conversion. Thus the induced losses are playing the role of the magnetic field.

As happens with many other optical and plasmonic analogues, the effect usually admits alternative explanations with no reference to the analogy. A possible model for our system is that the non-uniform losses break the symmetry of the system, so that the uncoupled eigenmodes of the structure are now diagonal modes $|V\rangle+|H\rangle$ and $|V\rangle-|H\rangle$, in accordance to the symmetry of the structure. The two modes show a different spatial profile owing to different exposures to the lossy region (see Fig. 2 
in [PAPER E]). An incoming linearly polarized light —as long as it is not polarized along the diagonal directions - can be expressed as a superposition of both diagonal polarizations and will excite both modes, and since their resonant frequencies are different, this will result in different phase responses for each component, resulting in polarization conversion of the incoming light.

\subsection{Our work: Electric analogue of Meissner levitation}
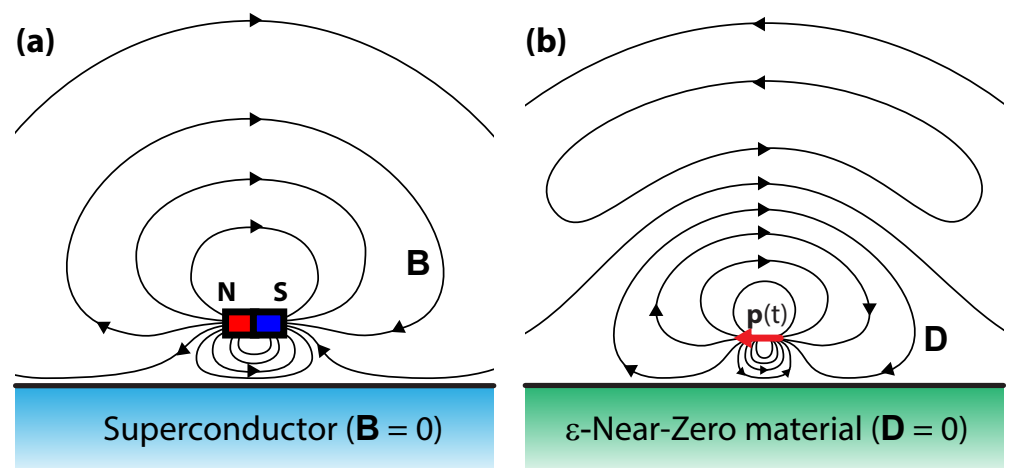

Figure 3.7: (a) Levitation due to the magnetic field expulsion of a superconductor due to the Meissner effect. (b) Analogous electric field expulsion of an $\varepsilon$-Near-Zero metamaterial.

The ability to levitate objects without direct contact has always been fascinating to humans. Several methods, including acoustic, aerodynamic, and electromagnetic ones exist to achieve this feat [209]. Of all methods, probably the most amazing of them is the stable levitation of magnets over superconductors (or viceversa) achieved thanks to the Meissner effect - the expulsion of a magnetic field by a superconductor [210]. Fig. 3.7(a) depicts a magnet levitating over a superconductor surface, showing the basic concept of the Meissner effect. The superconductor expels the magnetic flux density from its interior ${ }^{5}$. The hypotheses $\sigma \rightarrow \infty$ and $\mu=0$ do not achieve a complete description of the Meissner effect, which is quantum in nature, however, $\mu=0$ is occasionally used [211] to model some aspects of it, since it implies that the magnetic field must be expelled out of the material, with no component normal to the surface. This field expulsion exerts a magnetostatic gradient force on the magnet, which will levitate to a stable position.

Inspired by this notion, we envision an analogue scenario, shown in Fig. 3.7(b), where instead of the magnetic field being repelled by a superconductor - which

\footnotetext{
${ }^{5}$ The superconductor does not only behave as a perfect conductor by preventing a change in the magnetic field $\partial \mathbf{B} / \partial t=0$, but it also expels the magnetic field entirely $\mathbf{B}=0$.
} 
we can relate to $\mu=0,-$ now we have the electric field of a dipole being repelled from an $\varepsilon$-Near-Zero (ENZ) material, whose permittivity shows values $\varepsilon \approx 0$. Some natural ENZ materials exist at frequencies close to material resonances, such as silicon carbide [212] in the infrared, and they can also be engineered at any frequency using metamaterials. ENZ metamaterials ${ }^{6}$ have been studied in relation with a wide range of exciting applications [213-215], and are known to exhibit electric field expulsion from their interior. Thus, an electric dipole over a ENZ material constitutes an electric analog to the Meissner effect. However, the condition $\varepsilon=0$ is not realizable for static fields - while the Meissner effect is a static phenomenon-so our levitation mechanism acts upon harmonic sources of electric field, rather than static ones.

It should be noted that other metamaterial and plasmonic methods for levitation have been previously devised [69, 216-219], but our proposal is based on a different mechanism. A study of optical forces in particles near plasmonic materials was performed as early as 2003 [220] but the presented effect is not mentioned.

For our calculations, we made use of the Green function of a dipole radiating over a substrate of arbitrary permittivity, clearly described in Refs. [14, 17], to calculate the electric field. Then we used the expression of the full electrodynamic Lorentz force acting on a dipole, which can be greatly simplified, also described in Ref. [14]. After such calculations, we can retain only the dominant term at low heights, and we arrive at the following expression for the average vertical force experienced by a dipole placed at a distance $h$ over a material with relative permittivity $\varepsilon=\varepsilon^{\prime}+i \varepsilon^{\prime \prime}$ :

$$
F_{z}^{a v e}=\left(\frac{9}{512 \pi^{4} c_{0}}\right) \cdot p \cdot \mathfrak{R}\left(\frac{1-\varepsilon}{1+\varepsilon}\right) \cdot P_{\text {rad }} \cdot\left(\frac{h}{\lambda}\right)^{-4}
$$

where $c_{0}$ is the speed of light, $\mathfrak{R}$ denotes the real part, $P_{\text {rad }}$ is the power radiated by the dipole if it was placed in free space, and $p=1$ or 2 when the dipole is horizontal or vertical, respectively. The term $(h / \lambda)^{-4}$ indicates that the force is strong in the near field of the dipole, and is stronger the closer the dipole is to the surface. The term $\mathfrak{R}\{(1-\varepsilon) /(1+\varepsilon)\}$ determines the sign and strength of the force. For a lossless $\varepsilon$, we can see that if $-1<\varepsilon^{\prime}<1$, then the vertical force is positive, i.e. repulsive. This can be justified by the field expulsion analogy with the Meissner effect around $\varepsilon=0$, or even as analogous to conventional diamagnetic repulsion. The force actually tends to $\infty$ when $\varepsilon \rightarrow-1$, which is the surface plasmon resonance condition. However, this resonant effect quickly vanishes if small losses are added to the permittivity. Remarkably, however, the force that exists around $\varepsilon \approx 0$ remains repulsive even for high values of the losses -even higher than those of natural ENZ materials.

Thus, we present a method of electric levitation robust to losses, and also relatively robust to dispersion, since a wide range of values around $\varepsilon \approx 0$ achieve repulsive forces, corresponding to reasonably wide bandwidths. See [Paper 19].

\footnotetext{
${ }^{6}$ Two important properties of ENZ metamaterials are that they do not allow the accumulation of charges in their surfaces, thus the electric field must be parallel to them, and the electric displacement field inside them must be zero.
} 


\subsection{PAPER D}

Phys. Rev. B 81, 233101 (2010)

\section{Zero-bandwidth mode in a split-ring-resonator-loaded one-dimensional photonic crystal}

Francisco J. Rodríguez-Fortuño, Begoña Tomás-Navarro, Carlos García-Meca, Rubén Ortuño, Javier Martí, and Alejandro Martínez 
92 CHAPTER 3. PLASMONIC ANALOGUES OF QUANTUM PHENOMENA 


\title{
Zero-Bandwidth Mode in a Split-Ring-Resonator-Loaded One- Dimensional Photonic Crystal
}

\author{
Francisco J. Rodríguez-Fortuño, Begoña Tomás-Navarro, Carlos García-Meca, Rubén Ortuño, \\ Javier Martí and Alejandro Martínez
}

Valencia Nanophotonics Technology Center, Universidad Politécnica de Valencia, Camino de Vera s/n 46022 Valencia (Spain)

\begin{abstract}
We demonstrate that the resonance of metallic split-ring resonators can interact with Bragg phase interference effects in a surrounding one dimensional photonic crystal in such a way that a zero bandwidth mode arises within the photonic bandgap of the crystal. The band can also be designed to exhibit forward or backward slow light propagation. We present a very simple model to explain these phenomena and verify the results with numerical simulations. The dynamic tuning of the structure has potential for stopping light or timereversal applications.
\end{abstract}

Great advances in the control of electromagnetic fields by periodic structures have been made in recent years. On one side, metamaterials exhibit surprising material properties such as left-handed propagation [1], typically making use of split-ring resonators (SRRs) to achieve a negative permeability [2]. On the other side, photonic crystals (PhCs) make use of interference between incident and reflected waves to achieve exciting phenomena such as photonic bandgaps. Both structures can be used for slow-light propagation, which potentially allows the creation of optical buffers and delay lines, as well as enhanced nonlinear effects [3]. In this Report we combine both structures in order to achieve slow-light propagation; but we make use of phase interference effects between resonators. It is known that interference between excitation pathways lies behind the slow light phenomena observed in electromagnetically-induced transparency (EIT) [4] in atomic media: the strong dispersion arising due to quantum interference [5] yields a very slow group velocity around the transparency window. Classical analogues of EIT have been identified [6] and coupledresonator-induced transparency (CRIT) has been proposed [7], in which two nearby coupled optical resonators exhibit phase interference effects -analogous to EIT- resulting in slow light propagation. All those EIT/CRIT interference effects can be called direct, since the interfering entities share the same spatial location and their periodicity does not play a significant role. In contrast, indirect effects exist on resonators which are indirectly coupled via the propagating modes between them. This is the case in systems of periodically spaced optical resonators, named RPBGs (resonant photonic bandgap structures) [8] or indirect-CROWs (indirect coupled resonator optical waveguides) [9], on which the high dispersion around resonance due to phase interference effects yields a very low group velocity of light resembling EIT and CRIT- under the condition that the resonance frequency of the resonators matches a Bragg frequency of the optical lattice. Analogous structures have also been analyzed using interface response theory [10].

A fundamental limit of static slow-light devices is the delay-bandwidth product [11] which limits the available bandwidth of the pulses that can be delayed. This limit can be overcome -with certain limitations [12]- by using dynamic structures. The dynamic reduction of the group velocity down to zero, for light storage overcoming the delay-bandwidth product 
limitation, has been performed in atomic EIT schemes [13] as well as proposed in classical EIT analogues $[14,15]$ and also in periodically spaced resonators (RPBGs/indirect-CROWs) implemented with Bragg-spaced quantum well resonators [16-18].



FIG. 1. (Color online). Unit cell of the SRR-loaded PhC.

In this Report, we combine the worlds of PhCs and subwavelength metamaterials to implement a periodic structure exhibiting indirect interference effects which can result in slow light. In particular, we analyze the electromagnetic field propagation through an infinite SRR-loaded $\mathrm{PhC}$, whose unit cell is shown in Fig. 1. We only consider onedimensional propagation with the polarization as indicated in Fig. 1. We proceed in the same way as Syms et. al. [19]: the SRR is modeled as a simple LC circuit, coupled by a mutual inductance $M$ to a transmission line element representing the surrounding medium. We model the $\mathrm{PhC}$ by including finite length transmission lines that represent the two alternating $\mathrm{PhC}$ layers, through which incident and reflected waves will propagate and will indirectly couple the SRRs between them; such model is a simple means to account for Bragg effects. These effects will lead to very interesting slow light phenomena as will be shown below. Figure 2(a) shows the proposed model for the unit cell. Its transmission matrix $\mathbf{T}$ relates the output fields $E_{x}$ and $H_{y}$ with those at the input, and can be written as:

$$
\begin{gathered}
\mathbf{T}=\mathbf{T L}\left(\varepsilon_{2}, \mu_{2}, a_{2}\right) \cdot \mathbf{T L}\left(\varepsilon_{1}, \mu_{1}, a_{1}(1-p)\right) . \\
\quad \mathbf{S R R}(L, C, M) \cdot \mathbf{T L}\left(\varepsilon_{1}, \mu_{1}, a_{1} p\right) .
\end{gathered}
$$

where $\mathbf{T L}\left(\varepsilon_{i}, \mu_{i}, a_{i}\right)$ represents the transmission matrix of a transmission line with propagation constant $\beta_{i}=\omega \sqrt{\mu_{i} \varepsilon_{i}}$, characteristic impedance $Z_{i}=\sqrt{\mu_{i} / \varepsilon_{i}}$ and length $a_{i}, p$ describes the relative position of the SRR in the $\operatorname{PhC}$ medium, and $\operatorname{SRR}(L, C, M)$ represents the transmission matrix of a SRR [1,19]. Equating $2 \cos (k a)$ with the sum of the diagonal elements of $\mathbf{T}$ yields the dispersion relation of the periodic structure [20], given by

$$
\begin{aligned}
& 2 \cos (k a)=f(\omega) \\
& =f_{P h C}(\omega)+\frac{\omega_{0} M^{2}}{L}\left(\frac{\omega / \omega_{0}}{1-\omega_{0}^{2} / \omega^{2}}\right) \cdot C(\omega)
\end{aligned}
$$

where $k$ is the Bloch wavevector, $a=a_{1}+a_{2}$ is the periodicity of the unit cell, $\omega_{0}=1 / \sqrt{L C}$ is the resonance frequency of the SRRs, and the terms $f_{P h C}(\omega)$ and $C(\omega)$ [21] do not depend 
on the SRR response. This model could be also applied to other resonant particles such as high index dielectric disks or rings, whose circuit model is equivalent to that of the SRRs.
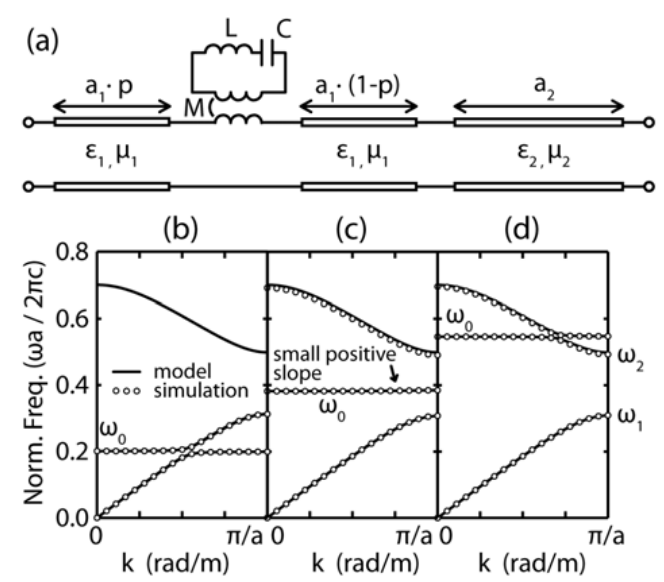

FIG. 2. (a) Proposed circuit model for the SRR-loaded PhC unit cell. (b-d) Modeled (solid lines) and simulated (circles) normalized dispersion relations for (b) $\omega_{0}<\omega_{1}$, (c) $\omega_{1}<\omega_{0}<\omega_{2}$, and (d) $\omega_{0}>\omega_{2}$. The model parameters are $p=0.5$, (b) $\omega_{0} a / 2 \pi c=0.2$ (c) $\omega_{0} a / 2 \pi c=0.381$ (d) $\omega_{0} a / 2 \pi c=0.5463$ and $\omega_{0} M^{2} / L=5$ in all cases. The PhC has $a_{1}=70 a / 75$ of air and $a_{2}=5 a / 75$ of dielectric $n_{2}=3.45$. The simulated SRR structure has $d_{\text {out }}=22 s, d_{\text {in }}=18 s, g=w=1 s, h=9 s$ and $a_{x}=a_{y}=100 s$, where $s$ is a reference feature size (b) $s=a / 75$, (c) $s=0.5 a / 75$ (d) $s=0.35 a / 75$.

Figures 2(b-d) show the dispersion relation described by the model (2) for three different values of $\omega_{0}$ with respect to the lower $\omega_{1}$ and upper $\omega_{2}$ edges of the PhC bandgap. When $\omega_{0}<\omega_{1}$ [Fig. 2(b)], we are in the long wavelength regime and the dispersion relation is very similar to that of a metamaterial SRR medium. When $\omega_{0}>\omega_{2}$ [Fig. 2(d)], the bands are similar to the previous case, but folded back into the first Brillouin zone. The most interesting case occurs when $\omega_{1}<\omega_{0}<\omega_{2}$ [Fig. 2(c) and Figs. 3(a-b)], where the SRR resonance occurs within the photonic bandgap. This resembles the setup of SRRs introduced in a bandgap created by a wire medium [22], a waveguide below cutoff [23] or an etched microstrip ground plane [24]. However, in the present case we cannot apply an effective medium interpretation. We interpret our results by noting that the SRR resonant mode does not coexist with propagating modes in the $\mathrm{PhC}$, and thus, neither coupling nor anticrossing take place, resulting in a flat dispersion relation with very small group velocity within the whole Brillouin region. A zoom into the passband, showing the complex Bloch wavevector $(k=\beta-j \alpha)$, is shown in Fig. 3(b). The band has an inflection point at which the group velocity dispersion is zero.

To check the model, Figs. 2(b-d) include dispersion relation simulations using the eigenmode solver of CST Microwave Studio. Fig. 3(c) shows a simulated transmission spectrum. Simulations show an almost exact agreement with the model. A source of disagreement is the slight redshift of the PhC bands when introducing the SRRs in simulations, specially the upper band, which can be interpreted as if the presence of the SRRs causes the PhC to "see" 
an effective medium with index $n_{\text {leff }}$ instead of $n_{1}$. This redshift becomes noticeable if the filling fraction of the SRRs is increased (larger SRR size or reduced transversal periodicity). Losses are an important practical issue: Fig. 3(b) shows, using dots, the complex dispersion relation when a resistance $R>0$ in series with $L$ and $C$ is used to account for metal absorption in the SRR, and Fig. 3(c) shows, using dots, a realistic transmission simulation for an arbitrarily chosen size ( $a=75 \mathrm{~mm}$ resulting in SRR resonance around $1.5 \mathrm{GHz}$ ), using a realistic copper metal for the SRRs. The results indicate that an experimental demonstration at microwave or terahertz frequencies is at hand.

(a)

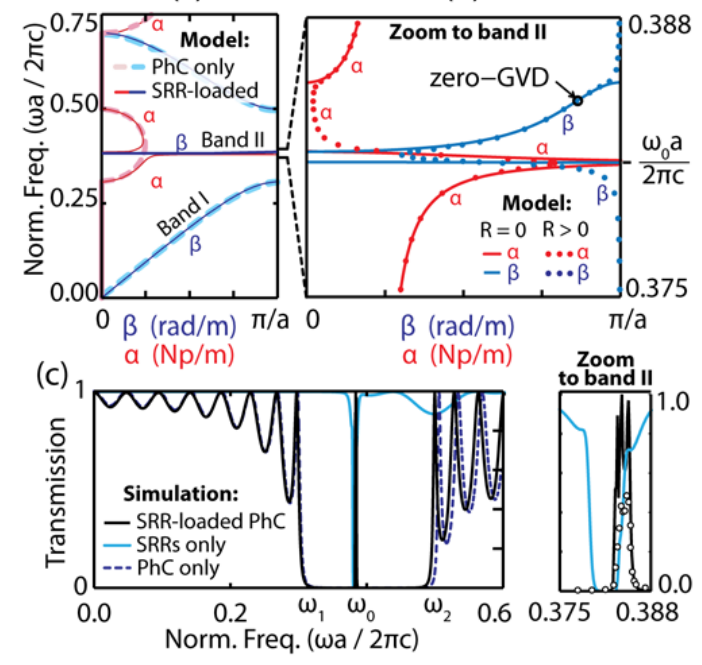

FIG. 3 (Color online). (a) Complex dispersion relation of the SRR-loaded PhC (thin solid line) and the PhC alone (thick dashed line) using same parameters as Fig. 2(c). (b) Zoom to Band II for the SRR-loaded PhC for lossless SRRs (solid lines) and lossy SRRs with $R / L=1 \times 10^{7} \mathrm{~s}^{-1}$ (dotted lines), a value chosen arbitrarily within the expected range. (c) Left: simulated power transmission spectrum through 8 unit cells of a lossless SRRloaded PhC, SRRs only and PhC only. Right: a zoom around $\omega_{0}$. Dots in zoomed view correspond to simulation with realistic copper metal $\rho_{C u}=5.8 \times 10^{7} \Omega^{-1} \mathrm{~m}^{-1}$ and $a=75 \mathrm{~mm}$.

Much more interesting phenomena related to the interaction of the SRRs with the PhC periodicity can be deduced by taking a closer look at the model. From Eq. (2) we see that a propagating band exists at each frequency $\omega$ only if $-2<f(\omega)<2$. The first term of $f(\omega)$ is $f_{P h C}(\omega)$, which constitutes the dispersion relation of the PhC without SRRs, and defines the PhC bandgaps whenever $\left|f_{P h C}(\omega)\right|>2$. The second term in $f(\omega)$ is due to the SRRs, and it is an asymptotic function which diverges at $\omega_{0}$. The total function $f(\omega)$ for the case $\omega_{1}<\omega_{0}<\omega_{2}$ is plotted in red (dark gray) in Fig. 4(a), where the three propagating bands previously shown in Figs. 2(c) and 3 are clearly observed. The width of the asymptote (and consequently the bandwidth of Band II) is determined by the product of the terms $\omega_{0} M^{2} / L$ and $C(\omega)$. The sign of the slope of the two branches of the asymptotic function (and consequently the sign of the slope of Band II) are determined directly by the sign of $C\left(\omega_{0}\right)$. 
Let us call threshold frequency $\omega_{t h}$ to the first frequency $\omega>0$ at which $C(\omega)$ changes sign [cyan (light gray) in Fig. 4(a)]. It follows that the slope and bandwidth of Band II is determined by the relative position of $\omega_{0}$ with respect to $\omega_{\text {th }}$. Therefore, Band II can be forward $\left(\omega_{0}<\omega_{t h}\right)$, backward $\left(\omega_{0}>\omega_{t h}\right)$ or completely flat with zero bandwidth $\left(\omega_{0} \rightarrow \omega_{t h}\right)$. Such result resembles EIT (and its analogues) as expected, since it arises from interference effects between resonators, but the form of $C(\omega)$ [21] suggests that in this case the phase interference is indirect (i.e. through propagating modes), in analogy to other indirect-CROW structures [8]-[10]. The effect can be interpreted as destructive field interference, taking place between the SRRs and the impinging propagating modes in the medium, that cancels out the total power flux. Unlike direct interference slow light schemes such as EIT, in this case only one resonator (plus periodicity) is required per unit cell. The phenomenon is illustrated in Fig. 5, in which the dispersion relation of Band II as well as its group index at the inflection point for varying values of $\omega_{0}$ around $\omega_{\text {th }}$ are shown. Simulations show the expected slight redshift of $\omega_{\text {th }}$ due to the previously mentioned change of $n_{1}$ into $n_{\text {leff }}$ [which affects $C(\omega)$ and thus $\left.\omega_{t h}\right]$.

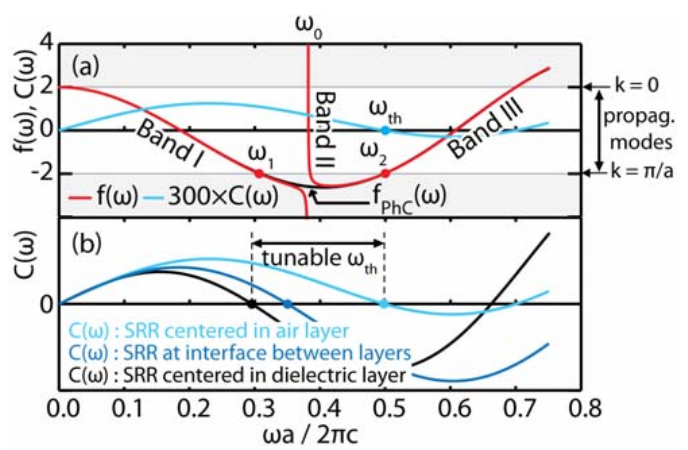

FIG. 4 (Color online). (a) Plot of $f(\omega), f_{P h C}(\omega)$ and $C(\omega)$ for the same parameters used in Fig. 2(c). (b) Plot of $C(\omega)$ for the same parameters varying the position $p$ of the SRRs.

We would like to highlight the difference between directly coupled resonator structures (standard CROWs [25]) and the case presented here (indirect-CROW) in which resonators are coupled through mode propagation in the medium, showing phase interference effects similar to those in EIT, thus allowing a decrease in group velocity (and consequently the bandwidth) down to zero. The comparison is analogous to that made by Khurgin [26] between standard CROWs and EIT media. Notice that magnetoinductive coupling between SRRs is not included in our model and does not seem to play a significant role.

An adiabatic dynamic tuning of the slope of Band II (for example, by modulating the index of the dielectric $n_{2}$, which in turn modifies $C(\omega)$ and thus $\omega_{t h}$ ) can lead to many sophisticated capabilities: the scheme satisfies the criteria identified by Yanik and Fan [27] for the stopping and storage of light pulses with no delay-bandwidth limitation [14] as well as the time-reversal of electromagnetic pulses [15]. Figure 4(b) shows how the term $C(\omega)$ and thus $\omega_{t h}$ can be tuned by varying the position of the SRRs within the unit cell (parameter $p$ in the model). As seen, this allows us to place $\omega_{t h}$ anywhere within the photonic bandgap. A value of $p \approx 0.2$ with the parameters used in Fig. 2 places $\omega_{t h}$ in the center of the bandgap. 
If the tunable band exists in the center of a PhC bandgap, a relatively fast adiabatic modulation of the refractive index (which is a critical practical consideration [12]) can be performed with negligible scattering into the neighboring bands [14]. In the particular case in which $a_{2}=0$ we have $C(\omega)=\sin \left(\beta_{1} a_{1}\right) / Z$, and the resulting structure is an example of RPBGs/indirect CROWs as studied in Refs. [8,9], showing an extremely slow group velocity band when the resonators are periodically spaced by a multiple of half wavelength. That phenomenon has been used in Bragg-spaced quantum-well resonators [16,17] to achieve slow-light applications, and the practical limitations have been thoroughly discussed [17], as well as the combination of slow-light with time-reversal capabilities to achieve distortion compensation in a reflection scheme [18]. All those considerations also apply to our proposed structure. Some advantages of our proposed metamaterial-inspired structure are the freedom in the design of the SRRs and in the number of achievable unit cells. In addition, we include the novelty of introducing a PhC (dielectric $n_{2}$ ). This increases the complexity of the phase interference phenomena between SRRs, allowing an increase in the bandwidth of the bandgap around the slow light band, and enables the tuning of $\omega_{t h}$ as previously discussed. Such apparent complexity arises simply from the in-line reflections produced by the PhC interfaces. In Ref. [28], the interaction between a cavity resonance and in-line reflections produced at both sides of the cavity is shown to result in complex interference phenomena that give rise to sharp and asymmetric Fano line shapes in the response. Our structure can be regarded as the simple periodization of such system. Fano lineshapes are therefore characteristic of SRR-loaded PhCs, showing a strong transmission dip at the resonance frequency always adjacent to the slow light band.

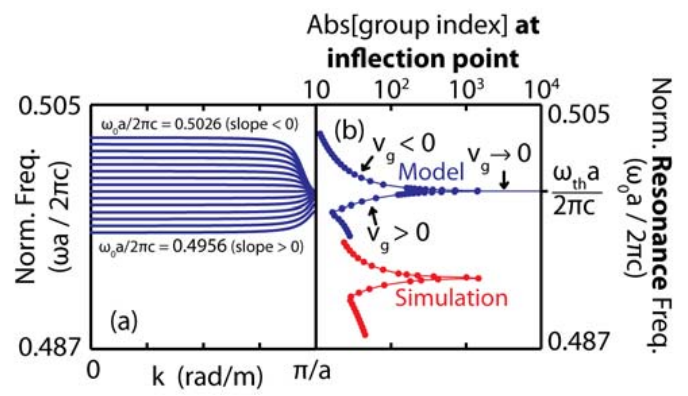

FIG. 5 (Color online) (a) Dispersion relation of Band II of the SRR-loaded PhC model for different values of resonant frequencies $\omega_{0}$ varied around a fixed $\omega_{t h}$. (b) Modeled (blue, darker) and simulated (red, lighter) absolute value of the group index of band II at its inflection point as a function of $\omega_{0}$.

In conclusion, we have modeled a structure exhibiting indirect phase interference effects between SRRs placed inside a PhC which result in a slow-light transmission band, confirmed with numerical simulations, which can ultimately show zero bandwidth. The result is similar to other slow light structures based on direct (EIT schemes) and indirect (RPBGs/indirectCROWs) interference. The zero bandwidth occurs when the resonance frequency $\omega_{0}$ of the resonators approaches the so-called threshold frequency $\omega_{t h}$, determined by the parameters of the $\mathrm{PhC}$ and the position of the resonators within the unit cell. The detuning of $\omega_{0}$ with respect to $\omega_{t h}$ determines whether the band has positive, negative or zero slope. An adiabatic 
tuning of such structure has potential for nontrivial capabilities such as stopping light with no delay-bandwidth product limitation and time-reversal operations.

We gratefully thank F. Falcone, M. Sorolla, M. Navarro-Cía and J.M. Escalante-Fernandez for their useful comments. Financial support by the Spanish MICINN under contract CONSOLIDER EMET (CSD2008-00066) is gratefully acknowledged. F.J. Rodríguez-Fortuño, C. García-Meca and R. Ortuño acknowledge financial support from grants FPI of Generalitat Valenciana, FPU of MICINN and FPI of Universidad Politécnica de Valencia, respectively.

\section{References:}

[1] L. Solymar and E. Shamonina, "Waves in metamaterials" (Oxford University Press US, 2009).

[2] J. B. Pendry, A. J. Holden, D. J. Robbins and W. J. Stewart, IEEE Trans. on Mic. Th. And Techniques. 47, 2075-2084, (1999).

[3] T. Baba, Nature Photon. 2, 465 (2008).

[4] S. E. Harris, Phys. Today 50, 36 (1997).

[5] Y. Q. Li and M. Xiao, Phys. Rev. A 51, 4959-4962 (1995).

[6] A. G. Litvak and M. D. Tokman, Phys. Rev. Lett. 88, 095003 (2002).

[7] D. D. Smith, H. Chang, K. A. Fuller, A. T. Rosenberger and R. W. Boyd, Phys. Rev. A 69, 063804 (2004).

[8] N. H. Kwong, J. Sipe, R. Binder, Z. Yang and A. Smirl, in Slow light: Science and Applications, edited by J. B. Khurgin and R. S. Tucker (CRC Press, 2009).

[9] Y. Xu, Y. Li, R. K. Lee, and A. Yariv, Phys. Rev. E 62, 7389-7404 (2000).

[10] J. O. Vasseur, A. Akjouj, L. Dobrzynski, B. Djafari-Rouhani and E. H. El Boudouti, Surface Science Reports, 54, 1-156 (2004).

[11] G. Lenz, B. J. Eggleton, C. K. Madsen and R. E. Slusher, IEEE J. Quantum Electron. 37, 525-532 (2001).

[12] J. B. Khurgin, Opt. Lett. 30, 2778-2780 (2005).

[13] D. F. Phillips, A. Fleischhauer, A. Mair, R. L. Walsworth and M. D. Lukin, Phys. Rev. Lett. 86, 783-786 (2001).

[14] M. F. Yanik, W. Suh, Z. Wang and S. Fan, Phys. Rev. Lett. 93, 233903 (2004).

[15] M. F. Yanik and S. Fan, Phys. Rev. Lett. 93, 173903 (2004).

[16] M. Hübner, J. Kuhl, T. Stroucken, A. Knorr, S. W. Koch, R. Hey and K. Ploog, Phys. Rev. Lett. 76, 4199-4202 (1996).

[17] Z. S. Yang, N. H. Kwong, R. Binder and Arthur L. Smirl, J. Opt. Soc. Am. B 22, 2144-2156 (2005).

[18] Z. S. Yang, N. H. Kwong, R. Binder and Arthur L. Smirl, Optics Letters 30, 2790-2792 (2005).

[19] R. R. A. Syms, E. Shamonina and V. Kalinin, J. Appl. Phys. 97, 064909 (2005). 
[20] L. Brillouin, “Wave propagation in periodic structures” (McGraw-Hill, London, 1946).

[21] The terms are given by

$$
\begin{gathered}
f_{P h C}(\omega)=2 \cos \left(\beta_{1} a_{1}+\beta_{2} a_{2}\right)+\left(2-\left(Z_{1} / Z_{2}\right)-\left(Z_{2} / Z_{1}\right)\right) \sin \left(\beta_{1} a_{1}\right) \sin \left(\beta_{2} a_{2}\right) \\
C(\omega)=\frac{1}{Z_{1}^{2} Z_{2}}\left\{Z_{1} Z_{2} \sin \left(\beta_{1} a_{1}\right) \cos \left(\beta_{2} a_{2}\right)+1 / 2 \sin \left(\beta_{2} a_{2}\right) .\right. \\
\left.\cdot\left[\left(Z_{1}^{2}+Z_{2}^{2}\right) \cos \left(\beta_{1} a_{1}\right)+\left(Z_{1}^{2}-Z_{2}^{2}\right) \cos \left(\beta_{1} a_{1}(1-2 p)\right)\right]\right\}
\end{gathered}
$$

[22] D. R. Smith, W. J. Padilla, D. C. Vier, S. C. Nemat-Nasser and S. Schultz, Phys. Rev. Lett. 84, 4184 (2000).

[23] R. Marqués, J. Martel, F. Mesa and F. Medina, Phys. Rev. Lett. 89, 183901 (2002).

[24] M. Navarro-Cía, F. Falcone, M. Beruete, I. Arnedo, J. Illescas, A. Marcotegui, M. A. G. Laso and T. Lopetegui, Micr. and Opt. Tech. Lett. 49, 2689-2692 (2007).

[25] A. Yariv, Y. Xu, R. K. Lee, and A. Scherer, Opt. Lett. 24, 711-713 (1999).

[26] J. B Khurgin, J. Opt. Soc. Am. B, 22 (2005).

[27] M. F. Yanik and S. Fan, Phys. Rev. A 71, 013803 (2005).

[28] S. Fan, Appl. Phys. Lett. 80, 908 (2002). 


\subsection{PAPER E}

Nano Lett. 12, 6309-6314 (2012)

\section{Analogue of the quantum Hanle effect and polarization conversion in non-Hermitian plasmonic metamaterials}

Pavel Ginzburg, Francisco J. Rodríguez-Fortuño, Alejandro Martínez, and Anatoly V. Zayats 


\title{
Analogue of the Quantum Hanle Effect and Polarization Conversion in Non-Hermitian Plasmonic Metamaterials
}

\author{
Pavel Ginzburg ${ }^{\ddagger 1 *}$, Francisco J. Rodríguez Fortuño ${ }^{\ddagger 1,2}$, Alejandro Martínez ${ }^{2}$ and Anatoly V. \\ Zayats $^{1}$
}

${ }^{1}$ Department of Physics, King's College London, Strand, London WC2R 2LS, United Kingdom

${ }^{2}$ Nanophotonics Technology Center. Universitat Politècnica de València. 46022 Valencia, Spain

\begin{abstract}
The Hanle effect, being one of the first manifestations of quantum theory introducing the concept of coherent superposition between pure states, plays a key role in numerous aspects of science varying from applicative spectroscopy to fundamental astrophysical investigations. Optical analogues of quantum effects help to achieve deeper understanding of quantum phenomena and, in turn, to develop cross-disciplinary approaches to realizations of new applications in photonics. Here we show that metallic nanostructures can be designed to exhibit a plasmonic analogue of the quantum Hanle effect and the associated polarization rotation. In the original Hanle effect, time-reversal symmetry is broken by a static magnetic field. We achieve this by introducing dissipative level crossing of localised surface plasmons due to nonuniform losses, designed using a non-Hermitian formulation of quantum mechanics. Such artificial plasmonic "atoms" have been shown to exhibit strong circular birefringence and circular dichroism which depends on the value of loss or gain in the metal-dielectric nanostructure.
\end{abstract}

The quantum Hanle effect describes the polarization rotation of scattered electromagnetic radiation due to atomic coherence between Zeeman states split by a magnetic field ${ }^{1}$. This phenomenon is a key in high-resolution atom spectroscopy ${ }^{2}$, detection of magnetic fields in solar prominence ${ }^{3}$ and stellar winds ${ }^{4}$. The main advantage in these measurements is a very high spectral resolution provided by the Hanle effect since the level crossing is not limited by the Doppler width of the spectral lines but solely by the coherence of the states in individual atoms, and sensitivity to magnetic fields. The observation of the effect is possible in the presence of a magnetic field which violates the time-reversal symmetry as was observed in the experiments on coherent backscattering 5 and can contribute to parity symmetry breaking ${ }^{6}$.

The investigations of optical analogues of quantum effects are important to achieve deeper understanding of quantum phenomena and give prospect to new applications based on cross-disciplinary approaches. For example, the realization of sharp spectral resonances with metallic (plasmonic) nanostructures important for biosensing and nonlinear photonic applications has resulted from the studies of optical counterparts of the Fano resonance, relying on the interference between scattering amplitudes of bound and continuum electronic states ${ }^{7}$, and electromagnetically induced transparency (EIT) due to destructive interference of electron probability amplitudes induced by two spectrally different optical beams ${ }^{8}$. 
In this Letter, we investigate an optical counterpart of the quantum Hanle effect. By employing the concepts of non-Hermitian quantum mechanics ${ }^{9,10}$, we have designed an artificial plasmonic "atom" which has a pair of degenerate resonances that split by broken time-reversal symmetry due to the presence of loss. This is a complete optical analogue of the atomic system where initially degenerate atomic states are split when the time-reversal symmetry is broken by a magnetic field ${ }^{1}$. The removal of degeneracy of the plasmonic resonances leads to very strong birefringence and circular dichroism, again in analogy with the quantum Hanle effect in atomic systems.
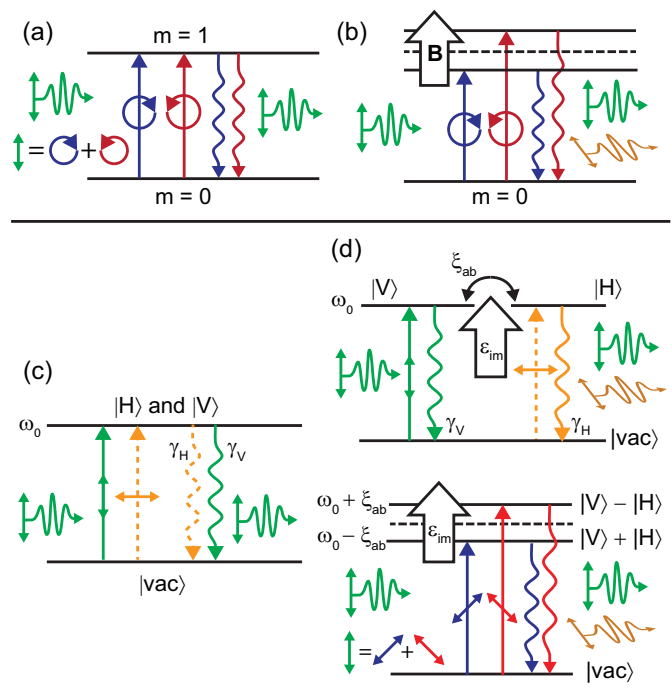

(e)

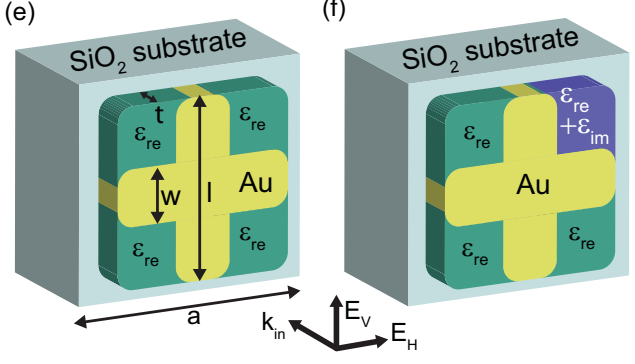

Figure 1. Quantum Hanle effect and its plasmonic analogue. (a-b) Basic level structure of 'traditional' Hanle effect (a) without and (b) with an applied magnetic field to break timereversal symmetry. (c-d) Optical counterpart of the Hanle effect in an artificial plasmonic atom: (c) Degenerate LSP resonances in the symmetric particle and (d) loss-induced coupling of the resonances $|\mathrm{H}\rangle$ and $|\mathrm{V}\rangle$ (top) and its equivalent scenario of the splitting into two supermodes $|\mathrm{V}\rangle+|\mathrm{H}\rangle$ and $|\mathrm{V}\rangle-|\mathrm{H}\rangle$ (bottom). (e,f) Schematics of the plasmonic metamaterial for the observation of the Hanle effect analogue: (e) a metal nanocross in a uniform embedding dielectric with degenerate LSP resonances, (f) one quadrant of the embedding dielectric is lossy leading to the splitting of LSP resonances of the otherwise symmetric metallic nanostructure. 
The basic scheme of the Hanle effect is represented in Fig. 1 (a-b). Linearly polarized light, being a superposition of left and right circular polarizations, coherently excites the p-orbitals of an atom, conserving the total angular momentum. The degeneracy between p-states [Fig. 1(a)] could be removed by an applied static magnetic field [Fig 1(b)]. The excited p-states decay in time with slightly dissimilar time constants, adding different phases for opposite circular polarizations and, as a consequence, resulting in polarization-unpreserved light scattering. The polarization of the scattered light depends then on the strength of the magnetic field which determines the splitting between states.

The plasmonic "atom level" diagram of our optical analogue is shown in Fig. 1 (c-d). We have considered a plasmonic "atom" which can be a metal nanoparticle with two localized surface plasmon (LSP) resonances. These resonances, in the quasistatic approximation valid for small nanoparticles, are solely determined by the particle's shape and surrounding environment and can be engineered and tuned to the desired frequency ${ }^{11-13}$. The vacuum state | vac > (no LSPs excited) represents the ground state of an atom. The degenerate in frequency LSP resonances corresponding to the excitation with different polarizations of the incident light are the analogues of the p-orbitals of the atom (marked as $|\mathrm{H}\rangle$ and $|\mathrm{V}\rangle$ on the diagram [Fig. 1(c)] for horizontal and vertical polarizations, respectively). The degeneracy of these LSP states can be removed by dissipative coupling in the presence of nonuniform loss [Fig. 1(d)], resulting in the shift of LSP resonances with respect to each other, as will be shown below.

The nanoparticle analysed in this work has been considered to be a metallic cross that has two degenerate dipolar LSP resonances corresponding to horizontal $(|\mathrm{H}\rangle)$ and vertical $(|\mathrm{V}\rangle)$ polarisations, respectively (Fig. 1(e)). This particle represents the above description if embedded in a uniform dielectric. In this degenerate case, the polarisation of the light normally incident on the nanostructure will not change upon reflection or in transmission. In principle, degeneracy may take place between plasmonic resonances of any order and could lead to interesting effects due to their interplay and time evolution ${ }^{14}$.

The degeneracy between the LSP resonances can be removed by introduction of losses $(\varepsilon \mathrm{im} \neq 0)$ in some locations near the cross arms (Fig. 1(f)). In this situation, the $|\mathrm{H}\rangle$ and $|\mathrm{V}\rangle$ modes of the particle are coupled giving rise to the uncoupled $|\mathrm{H}\rangle+|\mathrm{V}\rangle$ and $|\mathrm{H}\rangle-|\mathrm{V}\rangle$ modes excited by light with two orthogonal polarizations oriented at 45 degrees to the cross arms. These polarizations are always preserved after interaction with the structure due to its symmetry. Except for these light polarizations, such plasmonic nanoparticle with coupled $|\mathrm{H}\rangle$ and $|\mathrm{V}\rangle$ LSP states coherently scatters a fraction of the linearly polarised light into the orthogonal polarization state, leading to elliptization of linearly or circularly polarized incident light. Thus, the polarisation of the reflected/transmitted light will depend on the splitting of the LSP resonances governed by the loss - in analogy to the modification of the polarisation state of light scattered by atoms in the quantum Hanle effect depending on the splitting of atomic levels governed by magnetic field.

To analyze the loss-induced interplay and coupling between eigenmodes of the structure, we developed a rigorous theoretical description using the non-Hermitian quantum mechanical approach to describe loss-induced coupling between LSP resonances ${ }^{9,10}$. Non-Hermitian 
formulation of quantum mechanics is especially useful in the description of dissipative systems helping to get rid of 'bath' degrees of freedom. This formulation arises from the fact that probability conservation does not necessarily mean the hermiticity of time-evolution operators, but just the combination of parity and time-reversal commutations with an appropriate Hamiltonian of the system. Several interesting optical phenomena, such as higher harmonics generation ${ }^{15}$ or unidirectional mode coupling in waveguide structures ${ }^{16}$ have been analyzed in terms of this non-Hermitian formalism. For the plasmonic system under consideration, the eigenmodes can be derived from the solution of the following master equations ${ }^{17}$ :

$$
\begin{aligned}
& \Theta \vec{H}(\vec{r})=\left(\frac{\omega}{c}\right)^{2} \vec{H}(\vec{r}) \\
& \Theta(\vec{H}(\vec{r}))=\nabla \times\left[\frac{1}{\varepsilon(\vec{r}, \omega)} \nabla \times \vec{H}(\vec{r})\right],
\end{aligned}
$$

where $\vec{H}(\vec{r})$ is the magnetic field of the mode, $\omega$ is the angular frequency, $c$ is the speed of light in vacuum, and $\varepsilon(\vec{r}, \omega)$ is the position and frequency dependent dielectric constant. If both dispersion and absorption of the medium are neglected, the above defined $\Theta$-operator is Hermitian and forms a complete orthogonal set of eigenmodes $\{\vec{H}(\vec{r})\}$. Nevertheless, for certain problems, such as lasers, optical amplifiers or lossy structures, even if a single frequency is considered, the dielectric permittivity is a complex number and the $\Theta$-operator is not Hermitian anymore. Nevertheless, it is always possible to decompose it into a sum of the Hermitian and anti-Hermitian parts as follows:

$$
\begin{aligned}
& \Theta(\vec{H}(\vec{r}))=\Theta_{H}(\vec{H}(\vec{r}))+\Theta_{A}(\vec{H}(\vec{r})) \\
& \Theta_{H}(\vec{H}(\vec{r}))=\nabla \times\left[\frac{\varepsilon_{r}(\vec{r}, \omega)}{\varepsilon_{r}^{2}(\vec{r}, \omega)+\varepsilon_{i m}^{2}(\vec{r}, \omega)} \nabla \times \vec{H}(\vec{r})\right] \\
& \Theta_{A}(\vec{H}(\vec{r}))=-i \nabla \times\left[\frac{\varepsilon_{i m}(\vec{r}, \omega)}{\varepsilon_{r}^{2}(\vec{r}, \omega)+\varepsilon_{i m}^{2}(\vec{r}, \omega)} \nabla \times \vec{H}(\vec{r})\right],
\end{aligned}
$$

where $\Theta_{H}$ and $\Theta_{A}$ are the Hermitian and anti-Hermitian operators and $\varepsilon(\vec{r}, \omega)=\varepsilon_{r}(\vec{r}, \omega)+i \varepsilon_{i m}(\vec{r}, \omega) . \Theta_{H}$ provides an orthogonal set of eigenmodes spanning the entire electromagnetic space.

In the following, we have considered only two modes $|a\rangle$ and $|b\rangle$ and investigated their coupling originating from the presence of loss or gain $\left(\Theta_{A} \neq 0\right)$. The total magnetic field is given by the sum of the individual modes: 


$$
\vec{H}(\vec{r})=a(t) \overrightarrow{H_{a}}(\vec{r}) e^{i \omega t}+b(t) \overrightarrow{H_{b}}(\vec{r}) e^{i \omega t}
$$

where $a(t)$ and $b(t)$ are the complex time-dependent amplitudes of each mode. In the case of small imaginary part of the permittivity $\left(\varepsilon_{r}(\vec{r}, \omega)>>\varepsilon_{i m}(\vec{r}, \omega)\right)$, the time evolution can be obtained in the slowly varying amplitudes $(a(t), b(t))$ approximation by inserting Eq. 3 into Eq. 2 and taking into account the orthogonality of the field components $\left(\int \overrightarrow{H_{i}^{*}}(\vec{r}) \cdot \overrightarrow{H_{j}}(\vec{r}) d^{3} \vec{r}=\frac{\hbar \omega}{2 \mu_{0}} \delta_{i j}\right)$ as

$$
\begin{aligned}
& \frac{d a(t)}{d t}=-\xi_{a} a(t)-\xi_{a b} b(t) \\
& \xi_{a}=\frac{\varepsilon_{0}}{\hbar} \int \varepsilon_{i m}(\vec{r}) \overrightarrow{E_{a}^{*}}(\vec{r}) \overrightarrow{E_{a}}(\vec{r}) d^{3} \vec{r} \\
& \xi_{a b}=\frac{\varepsilon_{0}}{\hbar} \int \varepsilon_{i m}(\vec{r}) \overrightarrow{E_{a}^{*}}(\vec{r}) \overrightarrow{E_{b}}(\vec{r}) d^{3} \vec{r}
\end{aligned}
$$

, where $\overrightarrow{E_{j}}(\vec{r})$ is the electric field corresponding to the j-th mode and $\hbar$ is the reduced Plank constant, $\mu_{0}$ and $\varepsilon_{0}$ are the permeability and permittivity of free space. A similar equation may be obtained for the amplitude of the mode $b(t)$.

As can be seen from Eq. 4, the coupling between the two modes is determined by an overlap integral weighted by the position-dependent imaginary part of the permittivity. For symmetric structures with uniform losses, the coupling coefficients between two modes will be identically zero. However, the situation will be completely different if the medium is not uniformly lossy (e.g., selectively doped dielectric substrate or electrically or optically induced loss or gain). The loss-induced coupling will cause the removal of the degeneracy and will lead to the modification of the polarisation state of scattered light (Fig. 1(d)).

The transmission spectra of the structure were modelled as described in Methods. For the polarization of the incident light corresponding to the states $|H\rangle+|V\rangle$ and $|H\rangle-|V\rangle$ which remains unchanged after interaction with the nanostructure, single transmission minimum is observed in the absence of loss related to the excitation of the degenerate LSP modes associated with the cross-arms (Fig. 2). The characteristic field distribution near the plasmonic nanoparticle corresponds to the simultaneous excitation of two dipolar antennas.

When non-uniform losses are included, the states $|\mathrm{H}\rangle+|\mathrm{V}\rangle$ and $|\mathrm{H}\rangle-|\mathrm{V}\rangle$ become nondegenerate with the splitting increasing with the loss, and their field distribution is distinctly different (Fig. 2): one is symmetric due to the symmetry of the structure while the second one is antisymmetric with the field concentrated over the region with smaller loss. At the same time for linear polarizations $|\mathrm{V}\rangle$ and $|\mathrm{H}\rangle$ (Fig. 3(a)), the absorption resonance exhibits broadening and becomes asymmetric with the hint of the presence of two resonances (these can only be resolved in differential spectra). The fact that the two separate peaks are not clearly resolved in the transmission simulations for $|\mathrm{V}\rangle$ and $|\mathrm{H}\rangle$ is in agreement with the theoretical predictions. The width of the resonances is determined by the coefficients 
$\xi_{a}$ and $\xi_{b}$ (Eq. 4) which depend on both metal and dielectric losses, while the level splitting is defined by the coupling coefficient $\xi_{a b}$. Assuming a nonuniformly lossy dielectric environment $\left(\varepsilon_{i m}(\vec{r})>0\right)$, the weighted Hermitian inner product over $L^{2}$ metric space may be defined, and the Cauchy-Schwarz inequality will hold $\xi_{a} \xi_{b} \geq\left|\xi_{a b}\right|^{2}$. This implies that in this system the broadening of the resonances with the loss increase will always be greater than the splitting, preventing the direct observation of the splitting of the resonances. This is in striking contrast with the plasmonic analogues of EIT, where the splitting is larger than the resonance width ${ }^{8}$. In our case the coupling takes place between two "bright" modes while a "dark" (with narrow linewidth) mode is generally used for EIT.
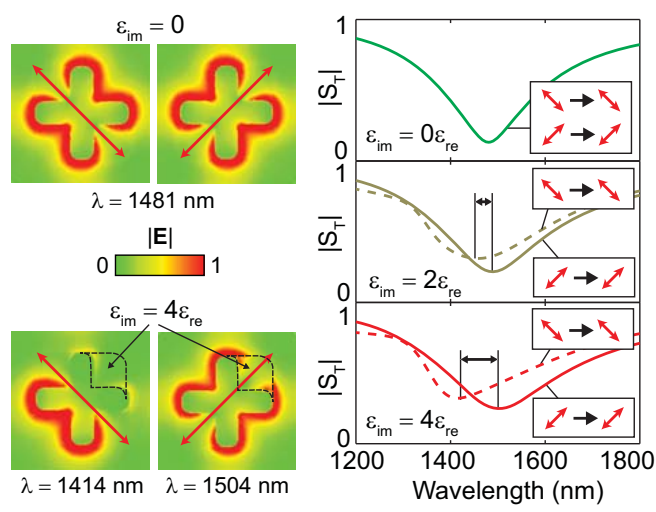

Figure 2. Removing degeneracy of LSP modes due to dissipative coupling. The spectra of transmission scattering parameters showing the absorption of the modes $|\mathrm{H}\rangle+|\mathrm{V}\rangle$ and $|\mathrm{H}\rangle-|\mathrm{V}\rangle$ of the nanocross (Fig. 1f). The polarization state of the modes cannot be modified by the nanostructure without nonuniform loss. The splitting of the degenerate LSP resonances is observed with the increase of the nonuniform loss in the dielectric. The electric field distribution in the nanostructure for degenerate and split resonances are shown next to the spectra. The parameters of the plasmonic structure marked in Fig. 1(e) were taken to be $\mathrm{w}=100 \mathrm{~nm}, \mathrm{t}=20 \mathrm{~nm}$, and $\mathrm{l}=400 \mathrm{~nm}$. For other parameters of the calculations see Methods.

Strong influence of such nanostructures on the polarization properties of light is expected as in the traditional Hanle effect due to the interplay of the resonances which respond differently to different incident light polarizations. The lower set of curves in Fig. 3(a) show the scattering efficiency of the input linear Ev or $\mathrm{EH}_{\mathrm{H}}$ polarization into the orthogonal polarization. This cross-polarization scattering leads to elliptisation of the incident linear polarised light upon reflection and in transmission. The overall conversion efficiency reaches $10 \%$ in the vicinity of the resonance in just a $20 \mathrm{~nm}$ thick layer of metamaterial and may be improved by increasing losses (Fig. 3(b)). 

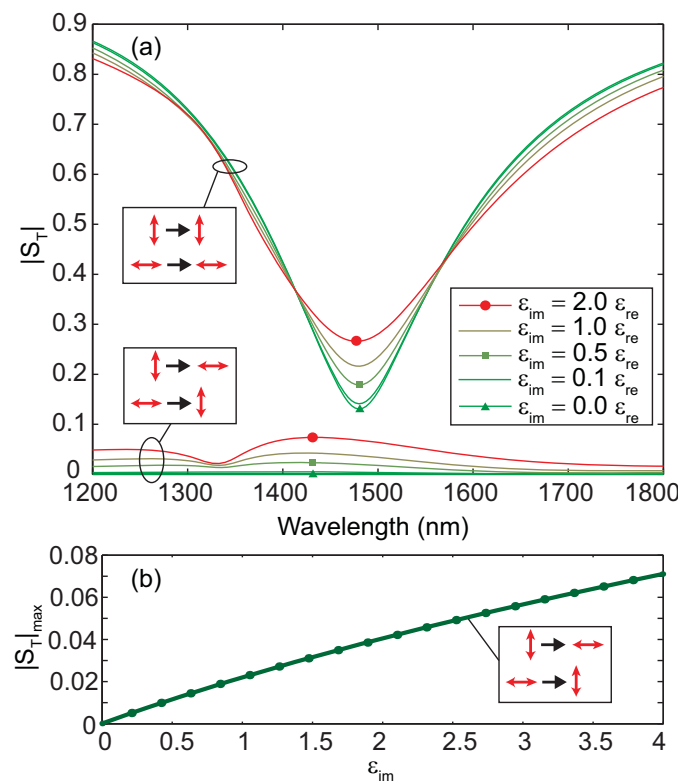

Figure 3. Polarization conversion due to the Hanle effect. (a) Spectral dependence of the scattering parameters $\mathrm{S}_{\text {т }}$ near the LSP resonance for different values of nonuniform loss. Upper set of curves correspond to the transmission of linearly polarized light $(\mid \mathrm{H}>$ or $\mid V>$ ) and is related to the absorption without polarization changes. Lower set of curves shows polarization conversion efficiency defined as the scattering parameter from one linear polarization $(|\mathrm{H}\rangle$ or $|\mathrm{V}\rangle)$ state into the orthogonal polarization state. (b) Dependence of the polarization conversion efficiency on the coupling loss. The parameters of the plasmonic structure are the same as in Fig. 2.

The steady state behaviour of the system may be analyzed in terms of 'rate equations' following the energy diagram in Fig. 1(d). The mode population of the vertical $(|\mathrm{V}\rangle)$ resonance under the incident $\mathrm{EH}$ (H-polarized) field can be obtained as

$$
\begin{aligned}
& V=\frac{\xi_{b a}\left(\gamma_{V, \text { rad }}+\gamma_{V, \text { dis }}\right)\left(\gamma_{H, \text { rad }}+\gamma_{H, \text { dis }}\right)}{\xi_{b a} \xi_{a b}\left(\gamma_{V, \text { rad }}+\gamma_{V, \text { dis }}\right)\left(\gamma_{H, \text { rad }}+\gamma_{H, \text { dis }}\right)-1} \kappa E_{H} \\
& \approx-\kappa \xi_{b a}\left(\gamma_{V, \text { rad }}+\gamma_{V, \text { dis }}\right)\left(\gamma_{H, \text { rad }}+\gamma_{H, \text { dis }}\right) E_{H}
\end{aligned}
$$

where Ен is the input " $\mathrm{H}$ "-polarized field amplitude, $\kappa$ is the excitation efficiency of the $|\mathrm{H}\rangle$ resonance under Eн illumination, and $\gamma_{V \text {,rad }}$ and $\gamma_{V \text {,dis }}$ are the radiative and nonradiative (dissipative) lifetimes of the resonance, respectively. In the approximation made in Eq. 5, we have assumed that the coupling $\xi_{a b}$ is small compared to the damping coefficient which is proportional to $\xi_{a}$ and $\xi_{b}$ (Eq. 4). The far-field intensity of light with the rotated polarization is proportional to $V / \gamma_{V, \text { rad }}$. The behaviour of the conversion efficiency with $\xi_{a b}$, predicted to be linear by Eq. 5, is verified by the numerical experiment for small losses where Eq. (5) is valid, and deviates slightly from linear dependence for larger losses (Fig. 3(b)). 
Finally, we have considered the effect of this structure on incident circularly polarized light. The amplitude ratio and the phase difference between the vertical and horizontal components transmitted through and reflected from the structure for left and right circular polarizations show that light experiences significant elliptisation, different for different handendess of the incident light (Fig. 4). The degree of eliptization also depends on how close the wavelength of the incident light is to the plasmonic resonances and on the loss.
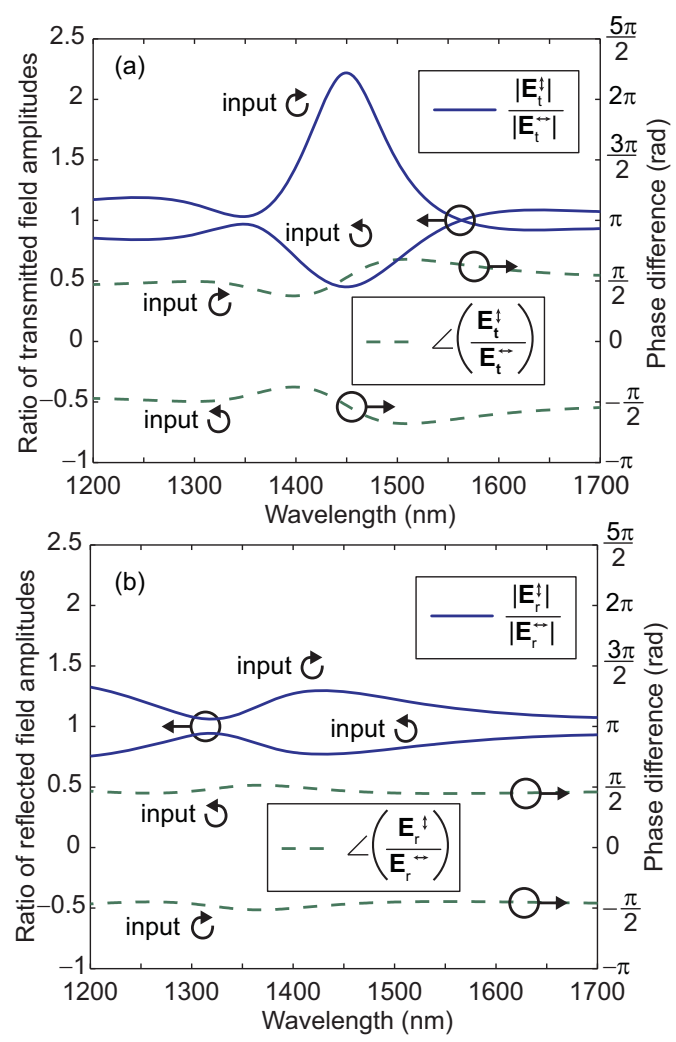

Figure 4. Circular dichroism in the non-Hermitian plasmonic metamaterial. Amplitude ratio (solid line) and phase difference (dashed line) of the Ev and Eн field components for (a) transmitted and (b) reflected light when the normally incident plane wave is right or left circularly polarized. The parameters of the plasmonic structure are the same as in Fig. 2.

We have introduced an analogue of the quantum Hanle effect in artificial plasmonic atoms. Ordered arrays of such plasmonic "atoms" have shown to form a metamaterial with extraordinarily pronounced circular birefringence and circular dichroism induced by the loss-coupled LSP states. The efficient control of the polarization state of light can be achieved using this effect in deep-subwavelength thick slabs (20 nm thickness) in both reflection and transmission geometries. 
Possible experimental tests of the proposed concept can be realised using lithographically defined plasmonic nanocrosses or, in complimentary geometry, cross-shaped apertures in a metal (plasmonic) film. The use of low-loss plasmonic material will be advantageous to provide highest possible differential contrast with the additional loss needed for coupling. The required spatially non-uniform losses can be implemented with selective doping of semiconductor coating ${ }^{18}$, selective polymerisation of the monomer cover layer ${ }^{19}$ or indeed controlled dynamically using electric-field, temperature or light induced absorption.

Polarization manipulation by 2D optically active artificially structured media has been previously demonstrated in several configurations, such as planar arrays of subwavelength gammadions ${ }^{20}$, plasmonic crystals ${ }^{21,22}$, spiral bull-eye structures ${ }^{23}$, and 3D metamaterials ${ }^{24-27}$. The use of the loss-coupled states open the possibility to build metamaterial components for active control of the reflected or transmitted light polarization if the nonuniform loss can be selectively induced by external stimuli, such as thermal, electric or optical signals. The optical response of the coupled nanocrosses resembles the response of nanoantennas recently used to achieve phase control and thus the control of refraction and reflection from the material interface structured in this way; ${ }^{28,29}$ thus, active control of loss-induced coupling may help to achieve active control over phase of reflected/transmitted light. If turned around, the effect can be used for measurements of local absorption (or gain) in metamaterials via polarization measurements or indeed in sensing applications ${ }^{30}$ for analytes introducing optical loss. Understanding of spatially nonuniform loss/gain coupling is imperative also for the development of loss-compensation and gain in metamaterials, where loss may result in additional and sometimes undesirable effects. From the fundamental point of view, the proposed general formalism for dissipative level crossing by employing the nonHermitian effects in metamaterials may be used to analyse and design new effects in plasmonic systems where metal loss is intrinsically present.

\section{Methods:}

In order to validate the analytical results, we have numerically simulated gold nanocrosses on a silica substrate, surrounded by a host dielectric medium. This surrounding material has been chosen to be selectively lossy only in one quadrant of the cross (Fig. 1(e)). For simulations, the particles were distributed in an ordered array with $600 \mathrm{~nm}$ periodicity. The particle dimensions (marked in Fig. 1(e)) were taken to be $\mathrm{w}=100 \mathrm{~nm}, \mathrm{t}=20 \mathrm{~nm}$, and $\mathrm{l}=400$ $\mathrm{nm}$. The refractive index of the substrate, as well as embedding dielectric is 1.45 , while the losses in one quarter of the embedding dielectric are subject to changes. A Drude model fit for gold was chosen for the particle's material ${ }^{31}$. The numerical modelling has been performed in the frequency domain using the commercial software CST Microwave Studio ${ }^{\mathrm{TM}}$ with periodic boundary conditions. The polarization resolved spectra of transmission and reflection scattering parameters from the array have been modeled at normal incidence. The transmission scattering parameters are defined so that $T=\left|S_{T}\right|^{2}$. In our case, we define two transmission parameters depending on the relative orientation of the light polarization considered at the input and the output, given by $\mathrm{S}_{\mathrm{T}}{ }^{\text {direct }}=\left(\mathrm{EH}^{\text {out }} / \eta_{\text {subs }^{1 / 2}}\right) /\left(\mathrm{EH}^{\text {in }} / \eta_{0^{1 / 2}}\right)=$ $\left(\mathrm{Ev}^{\text {out }} / \eta_{\text {subs }^{1 / 2}}\right) /\left(\mathrm{Ev}^{\text {in }} / \eta_{0^{1 / 2}}\right)$ and $\mathrm{ST}_{\mathrm{T}^{\text {cross }}}=\left(\mathrm{EH}^{\text {out }^{1 /}} / \eta_{\text {subs }^{1 / 2}}\right) /\left(\mathrm{Ev}^{\text {in }} / \eta_{0^{1 / 2}}\right)=\left(\mathrm{Ev}^{\text {out }} / \eta_{\text {subs }^{1 / 2}}\right) /\left(\mathrm{EH}^{\text {in }} / \eta_{0^{1 / 2}}\right)$ corresponding to the transmission of light with the same and orthogonal polarization, respectively. Ev and $\mathrm{EH}$ are the vertical and horizontal electric fields, respectively, and $\eta_{\text {subs }}$ and $\eta_{0}$ are the characteristic impedances of the substrate and vacuum. Since the scattering 
parameters are proportional to the electric field, the ratio of $\mathrm{ST}^{\text {cross }}$ and $\mathrm{ST}_{\mathrm{T}}{ }^{\text {direct }}$ is equal to the ratio of orthogonal polarization amplitudes at the output.

\section{Author Contributions and acknowledgements}

The manuscript was written through contributions of all authors. All authors have given approval to the final version of the manuscript. $\ddagger$ These authors contributed equally.

This work has been supported in part by EPSRC (UK). P. Ginzburg acknowledges Royal Society for a Newton International Fellowship. F. J. Rodríguez-Fortuño acknowledges support from grant FPI of GV and the Spanish MICINN under contracts CONSOLIDER EMET CSD2008-00066 and TEC2011-28664-C02-02.

\section{References}

1. Hanle, W. Über Magnetische Beeinflussung Der Polarisation Der Resonanzfluoreszenz. Z. Phys. A-Hadron. Nucl. 1924, 30, 93-105.

2. Colegrove, F.; Franken, P.; Lewis, R.; Sands, R. Novel Method of Spectroscopy With Applications to Precision Fine Structure Measurements. Phys. Rev. Lett. 1959, 3, 420422.

3. Stenflo, J. O. The Hanle Effect and the Diagnostics of Turbulent Magnetic Fields in the Solar Atmosphere. Sol. Physics 1982, 80, 209-226.

4. Ignace, R.; Nordsieck, K. H.; Cassinelli, J. P. The Hanle Effect as a Diagnostic of Magnetic Fields in Stellar Envelopes. I. Theoretical Results for Integrated Line Profiles. Astrophys. J. 1997, 486, 550-570.

5. Labeyrie, G.; Miniatura, C.; Müller, C.; Sigwarth, O.; Delande, D.; Kaiser, R. Hanle Effect in Coherent Backscattering. Phys. Rev. Lett. 2002, 89, 163901.

6. MacKintosh, F.; John, S. Coherent Backscattering of Light in the Presence of Timereversal-noninvariant and Parity-nonconserving Media. Phys. Rev. B 1988, 37, 18841897.

7. Luk'yanchuk, B.; Zheludev, N. I.; Maier, S. A.; Halas, N. J.; Nordlander, P.; Giessen, H.; Chong, C. T. The Fano Resonance in Plasmonic Nanostructures and Metamaterials. Nature Mater. 2010, 9, 707-715.

8. Liu, N.; Langguth, L.; Weiss, T.; Kästel, J.; Fleischhauer, M.; Pfau, T.; Giessen, H. Plasmonic Analogue of Electromagnetically Induced Transparency at the Drude Damping Limit. Nature Mater. 2009, 8, 758-762.

9. Moiseyev, N. Non-Hermitian Quantum Mechanics; Cambridge University Press, 2011.

10. Bender, C. M. Making Sense of non-Hermitian Hamiltonians. Rep. Prog. Phys. 2007, 70, 947-1018.

11. Berkovitch, N.; Ginzburg, P.; Orenstein, M. Concave Plasmonic Particles: Broad-band Geometrical Tunability in the Near-infrared. Nano Lett. 2010, 10, 1405-1408.

12. Prodan, E.; Radloff, C.; Halas, N. J.; Nordlander, P. A Hybridization Model for the Plasmon Response of Complex Nanostructures. Science 2003, 302, 419-422.

13. Berkovitch, N.; Ginzburg, P.; Orenstein, M. Nano-plasmonic Antennas in the Near Infrared Regime. J. Phys. Condens. Mat. 2012, 24, 073202. 
14. Ginzburg, P.; Berkovitch, N.; Nevet, A.; Shor, I.; Orenstein, M. Resonances Ondemand for Plasmonic Nano-particles. Nano Lett. 2011, 11, 2329-2333.

15. Gilary, I.; Kaprálová-Žd'ánská, P.; Moiseyev, N. Ab Initio Calculation of Harmonic Generation Spectra of Helium Using a Time-dependent non-Hermitian Formalism. Phys. Rev. A 2006, 74, 052505.

16. Ginzburg, P.; Hayat, A.; Vishnyakov, V.; Orenstein, M. Photonic Logic by Linear Unidirectional Interference. Opt. Express 2009, 17, 4251-4256.

17. Joannopoulos, J. D.; Johnson, S. G.; Winn, J. N.; Meade, R. D. Photonic Crystals: Molding the Flow of Light; Princeton University Press, 2008.

18. Asplund, C.; Mogg, S.; Plaine, G.; Salomonsson, F.; Chitica, N.; Hammar, M. Dopinginduced Losses in AlAs/GaAs Distributed Bragg Reflectors. J. Appl. Phys. 2001, 90, 794-800.

19. Eldada, L.; Shacklette, L. W. Advances in Polymer Integrated Optics. IEEE J. Sel. Topics in Quantum Electron. 2000, 6, 54-68.

20. Papakostas, A.; Potts, A.; Bagnall, D.; Prosvirnin, S.; Coles, H.; Zheludev, N. Optical Manifestations of Planar Chirality. Phys. Rev. Lett. 2003, 90, 107404.

21. Elliott, J.; Smolyaninov, I. I.; Zheludev, N. I.; Zayats, A. V. Polarization Control of Optical Transmission of a Periodic Array of Elliptical Nanoholes in a Metal Film. Opt. Lett. 2004, 29, 1414-1416.

22. Elliott, J.; Smolyaninov, I.; Zheludev, N.; Zayats, A. Wavelength Dependent Birefringence of Surface Plasmon Polaritonic Crystals. Phys. Rev. B 2004, 70, 233403.

23. Drezet, A.; Genet, C.; Laluet, J.-Y.; Ebbesen, T. W. Optical Chirality Without Optical Activity: How Surface Plasmons Give a Twist to Light. Opt. Express 2008, 16, 12559 12570.

24. Zhao, Y.; Alù, A. Manipulating Light Polarization with Ultrathin Plasmonic Metasurfaces. Phys. Rev. B 2011, 84, 205428.

25. Gansel, J. K.; Thiel, M.; Rill, M. S.; Decker, M.; Bade, K.; Saile, V.; Freymann, G. von; Linden, S.; Wegener, M. Gold Helix Photonic Metamaterial as Broadband Circular Polarizer. Science 2009, 325, 1513-1515.

26. Gansel, J. K.; Latzel, M.; Frolich, A.; Kaschke, J.; Thiel, M.; Wegener, M. Tapered Goldhelix Metamaterials as Improved Circular Polarizers. Appl. Phys. Lett. 2012, 100, 101109 .

27. Engheta, N. Circuits with Light at Nanoscales: Optical Nanocircuits Inspired by Metamaterials. Science 2007, 317, 1698-1702.

28. Ni, X.; Emani, N. K.; Kildishev, A. V.; Boltasseva, A.; Shalaev, V. M. Broadband Light Bending with Plasmonic Nanoantennas. Science 2012, 335, 427.

29. Yu, N.; Genevet, P.; Kats, M. A.; Aieta, F.; Tetienne, J.-P.; Capasso, F.; Gaburro, Z. Light Propagation with Phase Discontinuities: Generalized Laws of Reflection and Refraction. Science 2011, 334, 333-337.

30. Quidant, R.; Kreuzer, M. Biosensing: Plasmons Offer a Helping Hand. Nature Nanotech. 2010, 5, 762-763.

31. Johnson, P. B.; Christy, R. W. Optical Constants of the Noble Metals. Phys. Rev. B 1972, $6,4370-4379$. 
114 CHAPTER 3. PLASMONIC ANALOGUES OF QUANTUM PHENOMENA 


\section{Chapter 4}

\section{Plasmonic sensing}

THE DETECTION OF SMALL AMOUNTS of all kinds of chemical and biological substances - such as toxic chemicals in water or viruses in blood- has important applications ranging from environmental protection to food safety and medicine. In general, sensors are a way of detecting the presence of a certain substance, called the analyte. Sensors might have different requirements depending on their application. The need for a high sensitivity to achieve the successful detection of the analyte at concentrations as small as possible can be a critical parameter of a sensor, as well as its miniaturization and its low cost.

The high light field confinement which exists near metallic surfaces exhibiting plasmonic phenomena is an ideal method to achieve high sensitivity sensors, due to the strong interaction of the analyte with the plasmonic mode. Thus, one of the first applications of plasmonics, currently under commercial exploitation, is that of extremely sensitive biological and chemical sensing. In this chapter we give an introduction to the fundamentals of plasmonic sensing, and we describe and compare the two main types of plasmonic sensors, including a brief state of the art. The chapter serves as an introduction for our own theoretical and experimental contribution to plasmonic sensing given in [PAPER F, Paper 16].

\subsection{Fundamentals of plasmonic sensing}

Plasmonics provides an ideal platform for the label-free sensing of biological and chemical substances and particles. The mechanism behind plasmonic sensing is based on the shift of a plasmonic resonance induced by a change in the refractive index close to the metal surface, caused by the presence of the substance that we want to detect, the analyte. The detection of such change — by spectral measurement, intensity measurement at a single frequency, or other means- is an indication of the presence 
of the analyte. The performance of a plasmonic sensor is typically measured using the following parameters [13], allowing the objective comparison of different sensors:

- Sensitivity (S): measured in [nm/RIU], where RIU stands for refractive index unit. This parameter indicates the change in the resonance wavelength per unit refractive index change in the environment. In other words, $S=\partial \lambda_{\text {res }} / \partial n$ is the slope of the curve relating the resonant wavelength as a function of the index of refraction. Ideally, this parameter should be as high as possible, so that a small change in the refractive index translates into a big shift in the resonance frequency.

- Figure of Merit (FOM): defined as the sensitivity $S$ divided by the resonance bandwidth, e.g. the $-3 \mathrm{~dB}$ bandwidth. A high FOM is desired, because a given spectral shift is easier to detect the narrower the resonance.

Ideally only the analyte, and not other molecules, should come close to the metal surface and induce a change in the refractive index near it. A common way of achieving this is by performing a functionalization of the metal surface ${ }^{1}$. Functionalization consists on the adherence of a certain molecule to the metal surface, which in turn can bond specifically with the desired analyte, as shown in Fig. 4.4(b). This makes the analyte stay close to the metal surface, inducing a change in the plasmonic resonance.

\subsection{Types of plasmonic sensors and comparison}

Two main types of plasmonic sensors exist [25]:

- Sensors based on propagating surface plasmons (SPPs). In these sensors, a SPP wave is excited in a metal film and propagates along its surface, so that a small change in the refractive index above the metal surface - for example by depositing the analyte-gives rise to a change in the plasmon resonance frequency $\omega_{s p}$ and in the plasmon mode dispersion, as dictated by eq. (1.13). This can be detected by a change in intensity, wavelength, or angle of emission. These type of sensors are used commercially and present an extremely high sensitivity (of the order of $10^{6} \mathrm{~nm} / \mathrm{RIU}$ ), but they have a high cost: a commercial SPP sensor can cost around \$150.000 - \$300.000 [24].

- Sensors based on localized surface plasmon resonances (LSPRs) in metal nanoparticles. These kind of sensors do not exist commercially, but a high research interest is directed toward them due to their high potential and future commercial possibilities. The mechanism behind these sensors relies on the LSPR of metal nanoparticles, which depend not only in the size, shape and

\footnotetext{
${ }^{1}$ Other ways, such as physical adsorption, embedding in polymers, self-assembly, or Langmuir-Blodgett deposition, exist. For references see introduction to [PAPER F].
} 
material of the nanoparticle [221], but also on the material surrounding it [222]. By depositing the analyte close to the nanoparticles, the resonance frequency is modified, and the presence or absence of the analyte can be detected with a simple transmission or reflection measurement through the nanoparticle sample. This technique shows smaller sensitivities than commercial propagating SPP sensors (with sensitivities of the order of $10^{2} \mathrm{~nm} / \mathrm{RIU}$ ).

Despite the lower sensitivity, LSPR sensors present a series of noted advantages over propagating SPP sensors, which justifies the research interest in them [4, 24-26]:

- The electric field confinement in a LSPR is very high, around $1-2 \%$ of the wavelength [24], compared to that of propagating SPPs, around $10-15 \%$. This means that a very small quantity of analyte can occupy a great part of the entire modal field volume and achieve resonance shifts comparable to those of propagating SPP sensors despite the reduced sensitivity. This can be explained by considering the effective permittivity of the environment $\varepsilon_{\text {eff }}$ that a sensor perceives [223] when only a part of the environment $\varepsilon(\mathbf{r})$ is modified:

$$
\varepsilon_{\text {eff }}=\frac{1}{\int_{V}|\mathbf{E}(\mathbf{r})|^{2} d V} \cdot \int_{V} \varepsilon(\mathbf{r})|\mathbf{E}(\mathbf{r})|^{2} d V
$$

Since the sensitivity $S$ of the sensor is defined in terms of $\varepsilon_{\text {eff }}$ i.e. considering a change in the whole environment, the resonance shift will be $\Delta \lambda \approx S \cdot \Delta \varepsilon_{\text {eff }}^{1 / 2}$. In the LSPR there is a strong field enhancement on the so-called "hot spots", in which a small change of refractive index can have a dramatic effect in the resonance.

- The resonance frequency of nanoparticles is easily adjustable with the design of their size and shape [221], so the resonance can take place at any desired wavelength, leading to a greater freedom in the whole system (sources, detectors, etc. )

- LSPR sensors are very compact, with a much smaller size than propagating SPP sensors, and the sensing volume is smaller.

- The required instrumentation for LSPR sensing is simpler (it is enough to perform a transmission measurement through a substrate with nanoparticles) and better adapted to existing photonic technology. This greatly reduces the predicted cost of LSPR sensors, estimated at around \$5.000 [24].

- LSPR sensors can be designed to work with a single nanoparticle, at the expense of increased complexity in the equipment. Single nanoparticle sensing presents a series of advantages, such as a big sensitivity to extremely small quantities of analyte, among others [24]. The impressive possibility of detecting a single molecule of the analyte could be achievable. 
(a)

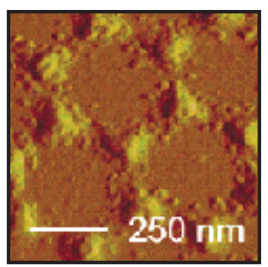

(b)

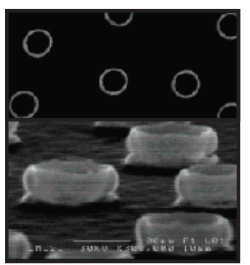

(c)

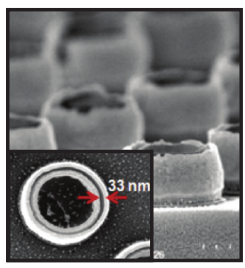

(d)

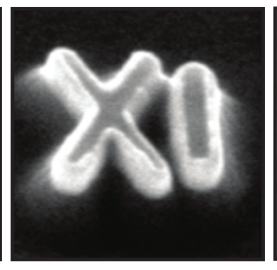

(e)

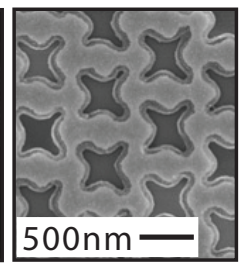

Figure 4.1: High sensitivity LSPR sensors taken from Refs. (a) [224], (b) [225], (c) [226], (d) [227] and (e) [228].

\subsection{State of the art of LSPR sensors}

Great advances have recently been made in LSPR sensing, as evidenced by the several review articles about the topic [4, 24-26]. There is a large volume of literature about this field. Below we briefly list some of the latest LSPR sensors which show particularly large sensitivities.

Initially, simple particles such as disks or holes in metal where used, with a moderate sensitivity around $200 \mathrm{~nm} /$ RIU. There are many studies on such simple particles. However, the sensitivity can be increased by increasing the aspect ratio of the particle and adding sharp edges or small gaps, which increases field enhancement, and brings about the creation of new improved designs, such as the nanotriangles [224] shown in Fig. 4.1(a), achieving a sensitivity of $300 \mathrm{~nm} /$ RIU. An even higher sensitivity of $800 \mathrm{~nm} / \mathrm{RIU}$ was achieved with tall nano-rings [225] as shown in Fig. 4.1(b). More recently, in 2011 a high sensitivity of $1075 \mathrm{~nm} /$ RIU was achieved using tall nanorings which additionally had a nanogap in their interior [226], as shown in Fig. 4.1(c). Sensitivities higher than $1000 \mathrm{~nm} / \mathrm{RIU}$ were also achieved using nanocrosses coupled to nanobars [227], shown in Fig. 4.1(d), which makes use of Fano interference to achieve a sharp peak (see section 3.1). Even more recently these values were improved by a design of nanocross holes in metal, as shown in Fig. 4.1(e), achieving a sensitivity of $1400 \mathrm{~nm} / \mathrm{RIU}$ [228].

Regarding the figure of merit, the typical values for LSPR sensors is well below 5 , due to the wide resonances arising from dissipation and radiation losses. This can be improved in several ways. One approach suggests using a thin gold film close to the LSPR particles [229]. Another very interesting approach is to make use of the sharp resonances provided by Fano metamaterials: a high FOM close to 6 was achieved with the structure shown in Fig. 4.2(a) [230]. The periodicity can also be used to reduce the resonance linewidth [231], and very recently in 2013 a FOM of 9 was experimentally demonstrated [232] with the structure shown in Fig. 4.2(b). As can be seen, the research is progressively advancing toward higher performance LSPR sensors which might soon be able to compete with commercial SPP sensors. 

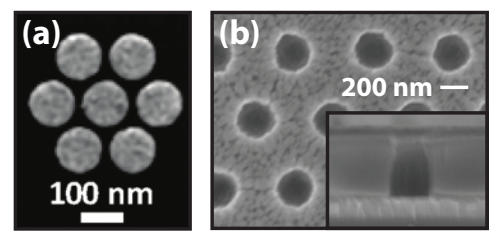

Figure 4.2: High figure of merit LSPR sensors (a) using Fano interference (taken from [230]), and (b) using periodicity (taken from [232]).

Although not purely an LSPR sensor, no revision of the state of the art is complete without the mention of the work of the group of A. V. Zayats, which combines concepts from both SPPs and LSPRs, using plasmons propagating along an effective medium of gold nanorods which themselves show localized resonances, and a sensitivity of $30.000 \mathrm{~nm} /$ RIU is achieved [233].

\subsection{Our work: Plasmonic nano-cross LSPR sensor}

In the literature, the designs with the better performances are usually the most difficult to fabricate: in particular, the design of particles with a high height-width ratio, or with very small gaps between metals, achieve good performances but are difficult to fabricate. Making use of the electron-beam lithography capabilities of the Nanophotonics Technology Center, we designed and fabricated relatively simple LSPR sensors consisting of metal nanoparticles over silicon or silica substrates that exhibit relatively high sensitivities.

Our first attempt was a simple nanodisk array [Paper 16] [see Fig. 4.3] on which we performed a novel functionalization of the silicon substrate rather than the metal. Since the field hot spots of a nanodisk are close to the edge of the metal with the

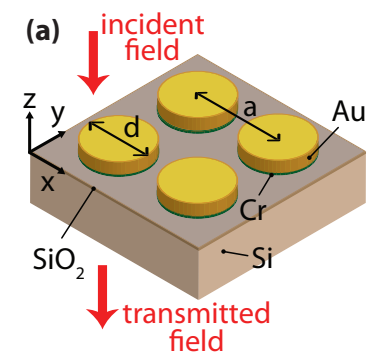

(b)

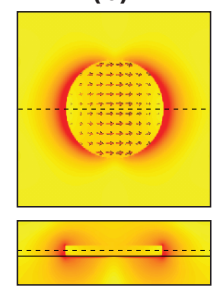

(c)

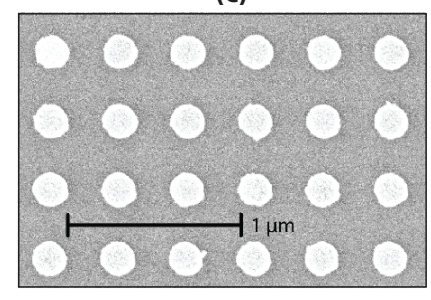

Figure 4.3: Nanodisk LSPR sensor taken from our [Paper 16]. (a) Geometric depiction. (b) Simulated resonant mode (c) Scanning electron micrograph image of the fabricated sample. 
(a)

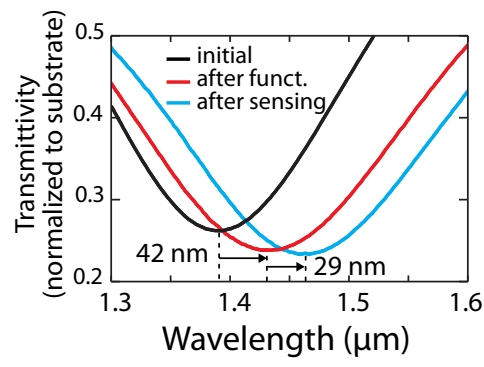

(b)

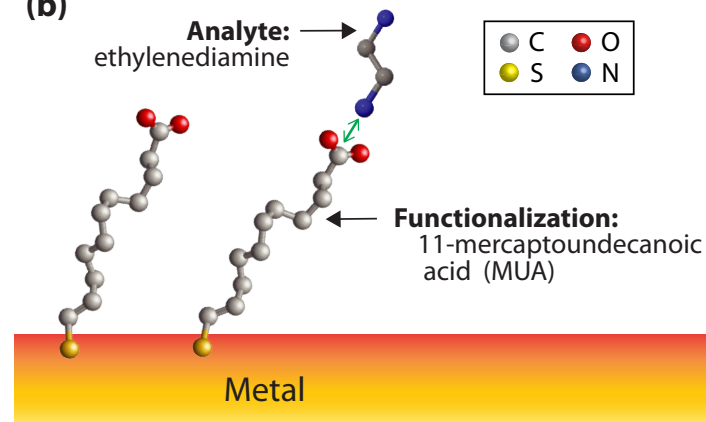

Figure 4.4: Functionalization of the metal surface for the adherence of the desired analyte. (a) Measured spectra in [PAPER F] after functionalization and sensing. (b) Example of functionalization molecules showing their adherence to the metal and to the analyte molecules. The chemicals depicted in this example correspond to those used in [PAPER F].

substrate, the functionalization of the substrate was a succesful way of bringing the analyte close to the resonant field volume. Certain advantages, such as re-usability of the sensor, were obtained thanks to the functionalization of silicon instead of the metal.

Once familiar with the fabrication and characterization process, we designed numerically a simple but sensitive nanoparticle. We wanted to increase the sensitivity by increasing the aspect ratio, as predicted by Mie theory [221,222, 234], so we designed gold nanocrosses on a silica substrate with predicted relatively high sensitivities of $500-700 \mathrm{~nm} / \mathrm{RIU}$ (for details of the geometry and simulated fields, see [PAPER F]). We fabricated them and measured their performance. The experimental results of the transmission measurements are shown in Fig. 4.4(a). This time we performed functionalization of the metal surface, using 11-mercaptoundecanoic acid (MUA) which adheres to gold on one end and attracts the analyte, ethylenediamine, on the other end. This is shown in Fig. 4.4(b). A relatively high sensitivity was thus achieved with a simple structure. All the details of the work are provided in [PAPER F].

\subsection{Our work: Nanoparticles on a silicon waveguide}

During this thesis, we had the idea of a system in which metallic nanoparticles exhibiting LSPR resonances were placed on top of a nanophotonic silicon optical waveguide. Numerical simulations proved that the light energy coming from the waveguide could excite the localized plasmonic resonances of the particles on the top. This should in principle be easily detected by measuring the transmission spectra 

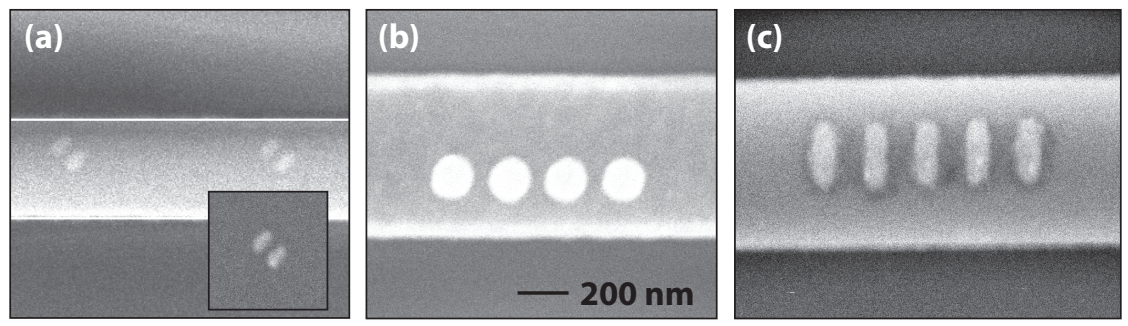

Figure 4.5: Scanning electron micrographs of the fabricated samples of metallic resonant nanoparticles on top of nanophotonic silicon optical waveguides. (a) Gold dipole pairs fabricated in 2010, (b) aluminium nanodisks fabricated in 2011 and (c) gold nanoellipses fabricated in 2012.

through the waveguides and observing extinction peaks at the LSPR wavelengths. Among other conceived applications, this could potentially lead to a simple method of single-nanoparticle sensing performed in parallel for several particles - by using different waveguides - integrated on a silicon chip, without the need of the advanced light focusing techniques typically used on single-nanoparticle sensing.

A significant amount of theoretical and experimental work was dedicated during this thesis to such device. The technological challenge for achieving the fabrication is considerable, because the waveguide and the nanoparticles are fabricated in different steps and need to be aligned with an extremely high precision. The adhesion of the metal to the silicon waveguide also has to be considered, usually requiring a chromium or titanium adhesion layer. Our fabrication team successfully surpassed those problems. Different metals and shapes were considered in simulations and experiments, Fig. 4.5 shows some of our fabricated structures. Many attempts to measure the structures where made, however, we did not manage to find the resonances expected from simulations in the transmission spectra of the waveguides. Our best fabrication result was published in a conference paper [Conf 23], but no journal publication has been written. During our attempts, however, another group published the same geometry, managing to show and measure the expected resonances [235-237]. To date we are attempting to replicate such published works, but have not yet succeeded. We currently suspect that the reason for our negative results is an excess of the thickness of the adhesion layer between the nanoparticle and the silicon waveguide. 


\subsection{PAPER F}

$$
\text { Appl. Phys. Lett. 98, } 133118 \text { (2011) }
$$

\section{Highly sensitive chemical detection in the infrared regime using plasmonic gold nanocrosses}

F. J. Rodríguez-Fortuño, M. Martínez-Marco, B. Tomás-Navarro, R. Ortuño, J. Martí, A. Martínez, and P. J. Rodríguez-Cantó 


\title{
Highly-sensitive chemical detection in the infrared regime using plasmonic gold nanocrosses
}

\author{
F. J. Rodríguez-Fortuño, M. Martínez-Marco, B. Tomás-Navarro, R. Ortuño, A. Martínez and \\ P. J. Rodríguez-Cantó
}

Nanophotonics Technology Center, Universidad Politécnica de Valencia, 46022 Valencia, Spain

\begin{abstract}
In this work, we report the design, fabrication and characterization of gold nanocrosses for chemosensing purposes. The nanocrosses are designed to exhibit a localized surface plasmon resonance which is very sensitive to refractive index changes of the surrounding medium, resulting in sensitivity values of around $500-700 \mathrm{~nm}$ per refractive index unit at wavelengths around $1.4 \mu \mathrm{m}$. We experimentally demonstrate the functionalization of the gold nanocrosses and the succesful sensing of chemical monolayers.
\end{abstract}

The applications of sensing devices are numerous, from environmental protection to medicine and food safety. The need for high sensitivity in order to detect smaller analyte concentrations is critical, since there exists a general tendency to miniaturize the sensor formats to facilitate detection of several substances on a same chip. Surface plasmon resonance (SPR) based sensors have proved to be a powerful technique for such applications. ${ }^{1}$ In particular, sensors based on localized surface plasmon resonances (LSPR) in metal nanoparticles ${ }^{2-6}$ are one of the preferred sensing platforms for gas detection and biosensing. LSPRs are responsible for a dip in the transmission spectra through arrays of (or single) metallic nanoparticles. The frequency of the LSPR depends on the size and shape of the nanoparticles, ${ }^{7}$ and is also extremely sensitive to the surrounding dielectric constant, so the presence of analytes close to the metal surface can be detected by monitoring the LSPR frequency. Different approaches for immobilization of molecules to the metal surface exist, such as physical adsorption, ${ }^{8,9}$ embedding in polymers, ${ }^{10}$ self-assembly, ${ }^{11,12}$ or LangmuirBlodgett deposition. ${ }^{13}$ Among them, use of self-assembly monolayers (SAMs) for coating metal surfaces (functionalization) minimizes the non-specific adsorption problem and introduces the possibility of having different functional end groups, providing a high degree of flexibility in the types of analytes that can be attached.6,11,14

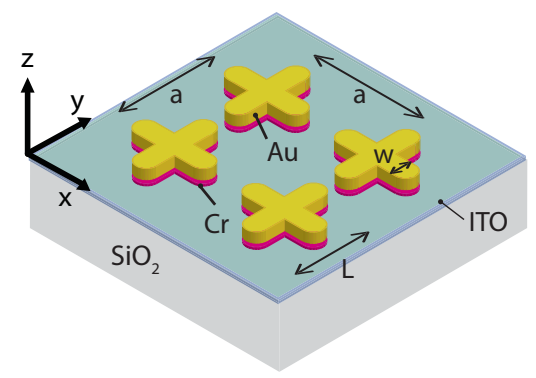

Figure 1. (Color online) Geometric depiction of four unit cells of the gold nanocross square array on a silica substrate. 


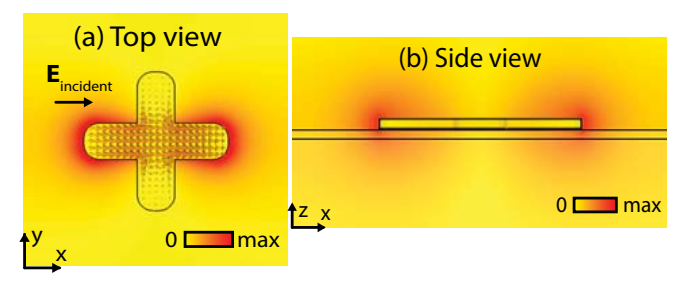

Figure 2. (Color online) Top (a) and side (b) views of the simulated electric field magnitude (color) and electric current density inside the metal (arrows) for the designed nanocrosses at their resonance frequency, excited with normal incident light whose electric field is polarized along the $x$-direction. Both views use a logarithmic scale in the field magnitude.

In this work, we report the design and fabrication of a LSPR-based nanostructure for chemosensing purposes. The structure designed consists of an array of metallic nanoparticles. In our previous work ${ }^{15}$ we used metallic nanodiscs on a silicon substrate. We now improve our previous design in two ways: Firstly, we use silica instead of silicon as the substrate, which has a lower refractive index, thus decreasing the confinement of the electric field in the substrate and increasing the sensitivity of the LSPR to local index changes in the exposed metal surface. Secondly, we use nanocrosses instead of nanodiscs to achieve a greater sensitivity of the LSPR and a narrower dip in the transmission spectrum. According to Mie theory, the sensitivity of the LSPR is greater for nanoparticles with higher aspect ratios, $7,16,17$ i.e. an elliptical nanoparticle has a greater sensitivity than a spherical one. Intuitively, a rectangular nanoparticle with high aspect ratio will have greater sensitivity than a circular one. The nanocross geometry is the combination of two rectangles perpendicular to each other, in order to achieve polarization independence while preserving an almost identical transmission spectrum and sensitivity. A geometric depiction of the structure is shown in Fig. 1. We modelled the structure with electromagnetic simulations (CST Microwave Studio). The thickness of the gold nanocrosses was $20 \mathrm{~nm}$. A $3 \mathrm{~nm}$ thick chrome layer and a $15 \mathrm{~nm}$ thick ITO layer, as seen in Fig. 1, were considered. Gold and chrome were modeled using Drude's model, with $\varepsilon_{\infty}=9(1)$ and $\omega_{p}=1.367 \times 10^{16} \mathrm{rad} / \mathrm{s}$ $\left(6.69 \times 10^{15} \mathrm{rad} / \mathrm{s}\right)$ for gold (chrome), $\gamma=1 \times 10^{14} \mathrm{~s}^{-1}$ in both and the refractive index of the ITO layer was taken to be 2.25. Structure dimensions are adjusted to fit the fabricated measured dimensions ( $L=365.9 \mathrm{~nm}, w=95.05 \mathrm{~nm}$ and $a=684.4 \mathrm{~nm}$ ). Fig. 2 shows the simulated electric field and electric current density of the LSPR excited with normal incident light. The nearfield coupling between neighbouring particles is small. Although the effect is small, ideally a large periodicity would result in the smallest spectral width, but for the LSPR to take place at frequencies below Wood's anomaly (so that no high-order diffraction modes are excited in the substrate) then we must restrict ourselves to an upper bound of the periodicity. We performed numerical simulations to study the sensitivity of the design, by varying the refractive index of the surrounding medium. The results are seen in Fig. 3, where we plot the transmittivity of the sample with different surrounding medium indices $n$. Note that higherorder plasmonic resonances, taking place below $1 \mu \mathrm{m}$ when $n=1$, appear at 1-1.3 $\mu \mathrm{m}$ for higher surrounding medium indices. We focus on the $1^{\text {st }}$ order resonance. We find that the sensitivity varies from $500 \mathrm{~nm}$ per refractive index unit (nm/RIU) when the surrounding medium is air, up to $740 \mathrm{~nm} / \mathrm{RIU}$ when $\mathrm{n}=2$. These values are greater than many other LSPR-based sensors found in the literature that make use of resonance shifts due to refractive 
index changes. ${ }^{2}$ This high sensitivity design was possible thanks to the electron beam lithography fabrication process, which offers a very precise control over the geometry of the metal nanoparticles, and therefore a great freedom in the design process. The calculated Figure of Merit [FOM = sensitivity (nm/RIU) / full-width-at-half-maximum (nm)] is also shown in Fig. 3, and varies between 2 and 2.2. The improvement in performance over our previous work involving nanodisks ${ }^{15}$ which showed a sensitivity of $84-170 \mathrm{~nm} / \mathrm{RIU}$ and a FOM between 0.19 and 0.34 , is significant.

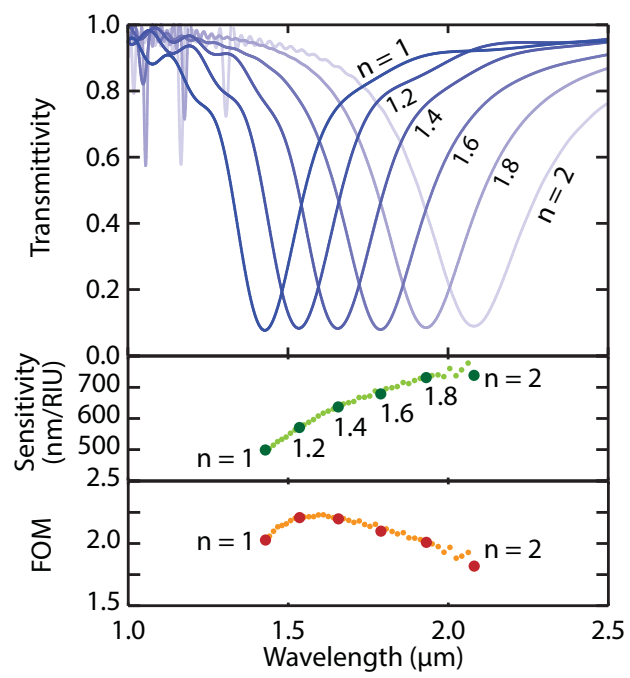

Figure 3. (Color online) Top: Numerical simulations of the transmittivity spectra of the gold nanocrosses array for different surrounding medium indices varying from $n=1$ to $n$ $=2$. Middle: associated sensitivity of the resonance frequency to index changes. Bottom: associated figure of merit.

Fabrication of the gold nanocrosses was performed on a silica substrate by standard electronbeam lithography with a single poly-methyl-methacrylate (PMMA). The silica surface was first coated with a $15 \mathrm{~nm}$ thin layer of ITO as conductive surface that suppresses charges during the electron-beam lithography as well as during the inspection process with techniques such as scanning electron microscopy (SEM). After developing of the resist, the sample was vacuum-deposited with a 1-2 $\mathrm{nm}$ chromium layer for adhesion purposes and followed by a $20 \mathrm{~nm}$ gold layer. A subsequent lift-off process removed the residual resist. Fig. 4 shows electron scanning microscope (SEM) images of the fabricated structure. The total sample area was $200 \times 200 \mu \mathrm{m}^{2}$. All the presented infrared (IR) transmission spectra were obtained using a Bruker ${ }^{\mathrm{TM}}$ Fourier Transform IR spectrometer. The IR illumination was focused on the sample with a $15 x$ objective with 0.4 numerical aperture, using square apertures in the microscope beam path to adjust the illumination region to the whole sample area ( 85000 nanocrosses), and the transmitted signal was collected with another microscope objective of same specifications on to a liquid nitrogen cooled mercury-cadmium telluride detector. The illumination was unpolarised. As can be seen in Fig. 5 the spectrum of the fabricated structure shows a good agreement with the simulation. 


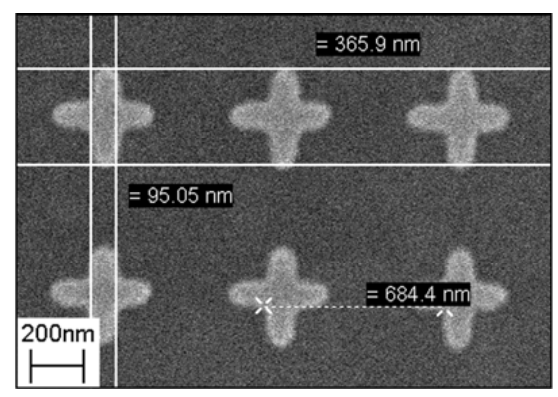

Figure 4. Scanning electron microscope (SEM) image of the fabricated sample.

After characterization of the spectrum, chemical functionalization of the gold surface was performed. Previously, the substrate was cleaned by rinsing with absolute ethanol (SigmaAldrich, 99.5\%) and deionized (DI) water. Formation of a $\mathrm{COOH}$ - terminated alkanethiol monolayer on the metal surface was accomplished by immersion of the sample in a $0.01 \mathrm{M}$ solution of 11-mercaptoundecanoic acid (MUA) (Sigma-Aldrich, 99\%) in absolute ethanol for 3 hours. The deposition process was carried out at room temperature. In order to remove the excess of reactants the sample was rinsed with absolute ethanol and DI water and dried under a flow of dry air. The formed monolayer is $1.7 \mathrm{~nm}$ thick having its thiol headgroup chemisorbed on the gold surface. Fig. 6 shows the power transmission spectrum before and after immersing the sample into the MUA solution. We can theoretically estimate the expected LSPR shift caused by this monolayer. From the simulated fields, we obtain a plasmon decay length at the tip of the nanocross arm of $l_{d}=29 \mathrm{~nm}$, from which we can estimate (taking the MUA refractive index to be 1.45) an effective index of the modified surface $^{18}$ of $\sim 1.06$. This calculation is a big simplification since the LSPR has a complex field distribution, and $l_{d}$ cannot be as clearly defined as in planar interfaces. Given the numerically obtained sensitivity of $500 \mathrm{~nm} / \mathrm{RIU}$, we expect a redshift of $30 \mathrm{~nm}$. In the experimental results, a redshift of $42 \mathrm{~nm}$ is observed, not too far from the estimation. After functionalization, sensing experiments were performed using ethylenediamine (SigmaAldrich, $75-80 \%)$ as target analyte, by immersion of the functionalized sample in a $10^{-4} \mathrm{M}$ ethylenediamine solution in DI water for 3 hours. The sample was then rinsed by DI water and dried under a flow of dry air to remove the unbound molecules. A time period of 3 hours was found satisfactory to provide a successful coupling of the analyte onto the sensor surface via amide coupling. ${ }^{19}$ As shown in Fig. 6, binding of the ethylenediamine molecules to carboxylic groups of the SAM led to a significant red-shift of $29 \mathrm{~nm}$ in the resonant wavelength of the nanocrosses. The red-shift is smaller than that measured for the functionalization layer, since the analyte monolayer attached to the functionalization layer is thinner and further away from the interface. The relative resonance shift $\Delta \lambda / \lambda_{0}$, being $\lambda_{0}$ the wavelength of the initial LSPR, has a value of 0.02 . 


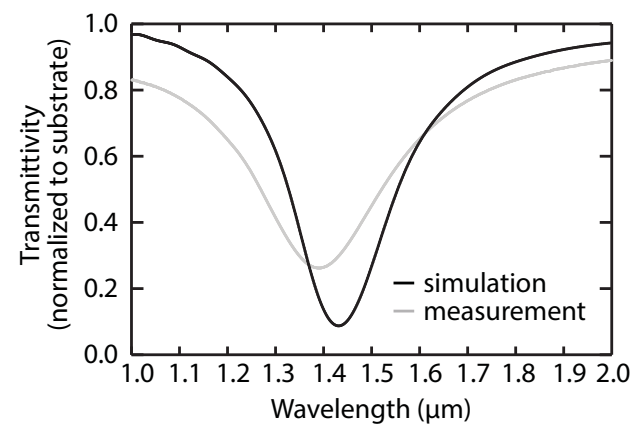

Figure 5. Simulated transmission spectra (black line) and measured IR transmission spectra (gray line).

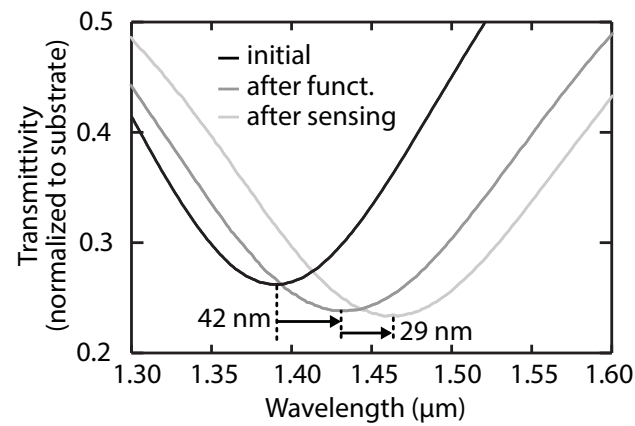

Figure 6. Measured IR transmission spectra before (black) and after functionalization of the gold surface (darker gray) and after sensing of ethylenediamine (lighter gray).

In conclusion, the freedom provided by lithographic fabrication processes allows the design of a sensor with a strong LSPR in the infrared regime and improved sensitivity. The gold surface was functionalized and further immersed in ethylenediamine aqueous solution, leading to a significant red-shift in the LSPR frequency of the gold nanocrosses, proving the monolayer sensing ability of the system.

Financial support by the Spanish MICINN under contracts CONSOLIDER EMET (CSD200800066) and TEC2008-06871-C02-02 is gratefully acknowledged.

\section{References}

[1] Jirí Homola, Anal. Bioanal. Chem. 377, 528 (2003).

[2] M. E. Stewart, C. R. Anderton, L. B. Thompson, J. Maria, S. K. Gray, J. A. Rogers, and R. G. Nuzzo, Chem. Rev. 108, 494 (2008).

[3] K. A. Willets and R. P. Van Duyne, Annu. Rev. Phys. Chem. 58, 267 (2007). 
[4] J. N. Anker, W. P. Hall, O. Lyandres, N. C. Shah, J. Zhao, and R. P. Van Duyne, Nat. Mater. 7, 442 (2008).

[5] J. Zhao, X. Zhang, C.R. Yonzon, A.J. Haes, and R.P. Van Duyne, Nanomedicine 1, 219 (2006).

[6] D.R. Shankaran, K.V. Gobi, and N. Miura, Sens. Actuators B 121, 158 (2007).

[7] K-S. Lee and M. A. El-Sayed, J. Phys. Chem. B 110, 19220 (2006).

[8] N. Miura, K. Ogata, G. Sakai, T. Uda, N. Yamazoe, Chem. Lett. 26, 713 (1997).

[9] D.R. Shankaran, K. Matsumoto, K. Toko, and N. Miura, Sens. Actuators B 114, 71 (2006).

[10] S. Cosnier, Biosens. Bioelectron. 14, 443 (1999).

[11] J.W. Lee, S.J. Sim, S.M. Cho, and J. Lee, Biosens. Bioelectron. 20, 1422 (2005).

[12] S. S. Mark, N. Sandhyarani, C. Zhu, C. Campagnolo, and C. A. Batt, Langmuir 20, 6808 (2004).

[13] K. Kato, C. M. Dooling, K. Shinbo, T. H. Richardson, F. Kaneko, R. Tregonning, M. O. Vysotsky, and C. A. hunter, Colloids Surf. A Physiochem. Eng. Aspects 198-200, 811 (2002).

[14] W. Senaratne, L. Andruzzi, and C. K. Ober, Biomacromolecules 6, 2427 (2005).

[15] P. J. Rodríguez, M. Martínez-Marco, F. J. Rodríguez-Fortuño, B. Tomás-Navarro, R. Ortuño, S. Peransí-Llopis, and A. Martínez, "Demonstration of near infrared gas sensing using gold nanodisks on functionalized silicon" (submitted).

[16] A. D. McFarland and R. P. Van Duyne, Nano Letters 3 (8), 1057 (2003).

[17] K. L. Kelly, E. Coronado, L. L. Zhao, and G. C. Schatz, J. Phys. Chem. B 107, 668 (2003).

[18] A. G. Brolo, R. Gordon, B. Leathem and K. L. Kavanagh, Langmuir 20, 4813-4815 (2004).

[19] T. Ramanathan, F.T. Fisher, R.S. Ruoff, and L.C. Brinson, Chem Mater. 17, 1290 (2005). 


\section{Chapter 5}

\section{Plasmonic interference phenomena}

ELECTROMAGNETIC INTERFERENCE is one of the most fundamental manifestations of the superposition principle in electromagnetism, which states that the field created at any point in space by a collection of charges and currents is equal to the sum of the fields created individually by each of them. This is possible thanks to the linearity ${ }^{1}$ of Maxwell equations. The interference between two or more coherent sources gives rise to a spatial field pattern which is given by the addition of the electromagnetic field originating from each source. Such addition can be constructive or destructive depending on the relative phase of the fields at each point.

Being an essential concept in many fields of physics, interference in electromagnetism has wide applicability for microscopy, spectroscopy, and communication technologies, among many others, and is the basis behind the concepts of reflection and refraction of light. Plasmonics, which stems from Maxwell equations, also exhibits interference phenomena which give rise to many interesting applications. In this chapter, some examples of advanced plasmonic applications making use of interference will be briefly presented, and it will serve as introduction to two of our works, [PAPER G, PAPER H], which make use of plasmonic interference to achieve two novel applications: loss reduction and unidirectional excitation.

\subsection{Some plasmonic applications of interference}

The phenomenon of interference is so ubiquitous in plasmonics that many of the applications already mentioned are related to it. For example, most of the plasmonic analogues of quantum phenomena presented in chapter 3 were based on interference,

\footnotetext{
${ }^{1}$ The superposition principle is true for the solutions of any linear differential equation.
} 

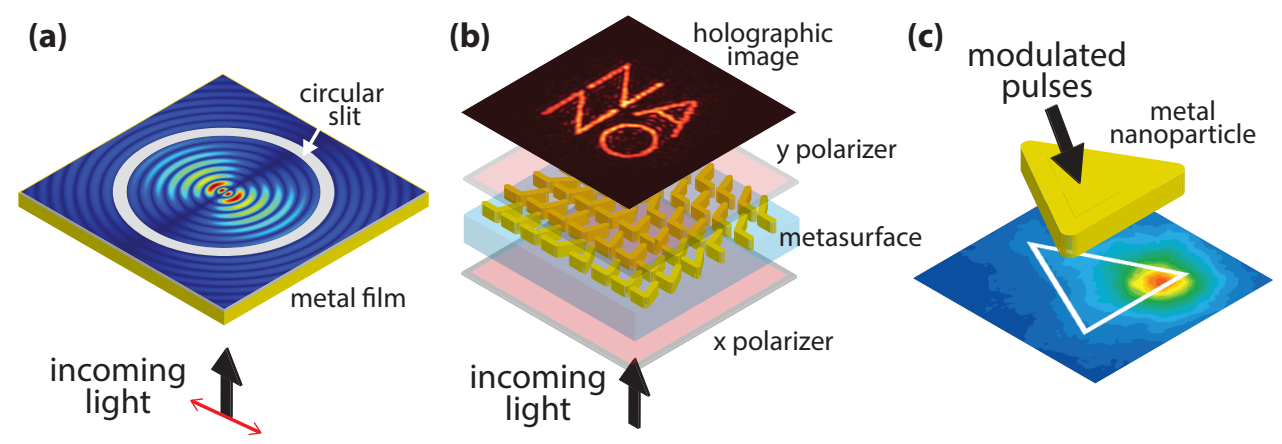

Figure 5.1: Some examples of plasmonic interference phenomena. (a) Interference of surface plasmons propagating on a metal surface [238]. (b) Holography using plasmonic metasurfaces [239]. (c) Near field interference for spatial energy localization in a plasmonic nanoparticle [240].

such as the Fano responses and the Plasmonic Induced Transparency. Here we mention some other novel plasmonic applications also related to interference. This is not meant to be a comprehensive compilation, because such a list would be too extensive, instead we present what we consider to be a variety of representative examples.

- A plasmonic interference pattern can be formed when multiple surface plasmon waves overlap coherently. The plasmons can be generated by illuminating structures milled in a metal film, such as edges, and very interesting interference patterns can be designed. Using circular or elliptical structures, the excited SPPs can be focused into a spot, forming a plasmonic lens [238] [see Fig. 5.1(a)], and the concept can be generalized to a broad-band manipulation of the twodimensional SPP pattern [241] with potential applications in nanolithography, particle manipulation, and other fields.

- Interference can also take place not between the SPPs themselves, but between the fields radiated or scattered by plasmonic structures. This can be used for focusing of light after interaction with the plasmonic structure [242], allowing nanolithography on a nearby sample using plasmonic structures [243]. If the phase of the scattered waves is fine-tuned by a plasmonic structure in a surface - a metasurface,- - the laws of refraction and reflection can be generalized [244], allowing all kinds of exciting applications such as broadband light bending [245], creation of optical vortices [246], rare out-of-plane refraction and reflection [247], and holography [239] [see Fig. 5.1(b)], all intimately related with interference. The same approach was conventionally used by engineers working at lower frequencies in the design of reflectarrays and transmitarrays [248]. 
- Interference can take place between the scattered fields of two objects. This was employed in the design of a plasmonic cover to cloak the scattering of another object, giving rise to the exciting possibility of a plasmonic invisibility cloak $[249,250]$ that can be designed for broadband operation [251].

- Other applications make use of interference taking place between the different modes propagating on a multimode waveguide, a well known phenomenon in optical dielectric waveguides [252] which is also observed in plasmonic waveguides [253]. Applications including plasmonic splitters [254, 255] and couplers [256] have been proposed. An active control of the index of one of the modes in a plasmonic waveguide exhibiting multimode interference can allow the switching between constructive and destructive interference at an output port. A remarkable experiment using such idea demonstrated the plasmonic equivalent of a transistor, named PlasMOStor [257]. Our contribution in [PAPER G] proposes the use of such multimode interference for plasmonic loss reduction.

\subsection{Our work: Plasmonic loss reduction using multi- mode interference}

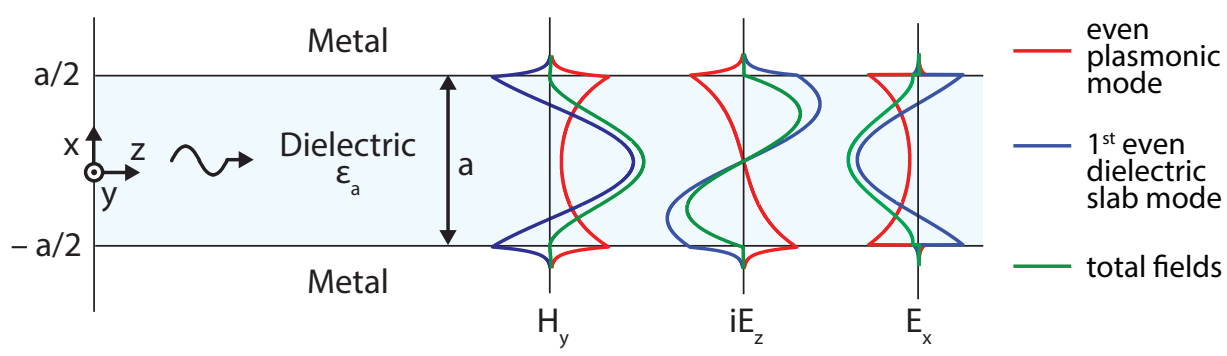

Figure 5.2: Plasmonic multi-mode interference in a metal-dielectric-metal waveguide to achieve reduction of losses.

Plasmonics allows unprecedented light confinement; however this comes at the cost of high losses. The unavoidable compromise that exists between high-confinement and low-loss is the basis for several figures of merit defined for plasmonic waveguides $[258,259]$. The search for low-loss plasmonic propagation has led to several hybrid plasmonic-dielectric waveguide proposals [260-264]. However losses are still an inherent part of plasmonic modes - the movement of electrons inevitably causes Joule heating - and the propagation lengths are on the order of microns. As a more direct approach to battle against low-loss, the need for better materials - e.g. low loss metals - has been pointed out [35], and metal alloys have been proposed to that end 
[265]. Another approach is to compensate the plasmonic losses with gain in active media [266, 267].

In our work [PAPER G] we propose a novel approach to low-loss plasmonic waveguides, by making use of multimode interference. The losses in plasmonic waveguides arise from the currents generated inside the metallic walls $(\mathbf{J}=\sigma \mathbf{E}$, where $\sigma$ is the metal conductivity). To reduce those losses, we propose to elliminate or reduce the amount of electric field inside the metal walls by using the interference between two or more waveguide modes. A cancellation of the fields within the metal walls leads to drastically reduced losses. Fig. 5.2 depicts the idea, showing the field profiles of two plasmonic modes in a metal-dielectric-metal waveguide, and the total fields after their addition. The interference shows largely reduced fields inside the metal, which reduces plasmonic loss. Two drawbacks to this method exist:

- The loss reduction is local, because due to the different propagation constants of the two modes $k_{z 1}$ and $k_{z 2}$, the relative phase between them will gradually change, so that destructive interference will eventually turn into constructive interference after half a beat length $L_{\pi}=\pi /\left(k_{z 1}-k_{z 2}\right)$. Thus the reduced losses occur for propagation from a point $A$ to a point $B$, separated a distance $L<2 L_{\pi}$, surrounding the point of minimum losses.

- The waveguides should be multimode and support at least two modes, thus we need to use relatively wide waveguides. Indeed, the wider the waveguide, the closer the two propagation constants of the two modes, and thus the longer the beat length. So, again, we arrive at a compromise between low-loss and high-confinement.

To study the phenomenon, we start by considering multimode interference in a parallel plate waveguide with finite conductivity walls. In such cases, the currents in the walls are responsible for the losses, and are proportional to the magnetic field parallel to the walls. Combining modes with equal symmetry can result in a zero magnetic field parallel to the walls. We derive a general expression for the effective losses - given by the integral of the losses in a length L of propagationin the conductor under such mode combination, valid for any conductivity and any frequency, which after normalizing by the loss of the TEM mode of the waveguide (which is the lowest loss mode) yields the loss improvement ratio, given in eq. (4) and plotted in Fig. 3 of [PAPER G].

Afterwards, the plasmonic case is considered, and the exact condition for minimizing the fields inside the metal, and therefore the losses, is given as a ratio of the amplitudes of each mode. With the adequate ratio of powers between the modes, a point of almost zero loss can be achieved. This is a remarkable fact: it means that at that point along the waveguide, the plasmonic modes are propagating with no electron movement in the walls. The effective attenuation coefficient of the plasmonic 
waveguide for a propagation length $\mathrm{L}$ around such low loss point is obtained as:

$$
\alpha_{\text {loss }}^{\text {eff }}=\alpha_{\text {loss }}^{\text {ave }} \cdot\left(1-\operatorname{sinc}\left(\frac{L}{2 L_{\pi}}\right)\right)
$$

where $\alpha_{\text {loss }}^{\text {ave }}$ is the weighed average loss coefficient of the two modes, and $\operatorname{sinc}(x)=$ $\sin (\pi x) /(\pi x)$. Thus, an effective loss approaching zero can be obtained for propagating lengths $\mathrm{L}$ small compared to the beat length of the two modes. All the details and derivations of this result are given in [PAPER G].

\subsection{Near field interference phenomena}

As previously mentioned, interference is the cornerstone for many applications and fundamental principles in optics. Usually it relies in the addition of two or more propagating beams which have undergone different propagation phase lags. In the field of nanophotonics, however, the distances involved can be so small that not only the propagating components interfere, but also the near-fields with their evanescent components (see section 2.3). For such evanescent components, no phase lag occurs.

The notion of near field interference was made popular by a paper of R. Merlin in 2003 [242], showing that by generating the appropriate field profile at some plane $z=0$, the wave can converge into a spot at a certain plane $z=f$. If the field profile at $z=0$ contains high-spatial-frequency Fourier components - corresponding to evanescent fields - then the focused spot can potentially be of subwavelength dimensions. The concept of near field interference is behind several lensing proposals for subwavelength focusing [8, 268-270].

Near field interference also allows a very surprising effect: by properly tuning the amplitude and phase of incoming pulses into a sample, spatial localization of the near-fields in the resonances of the sample can be achieved [271] this has been demonstrated in nanoparticle LSPR resonances [240] [see Fig. 5.1(c)] and has been used for the selection of propagation paths along an array of metal nanoparticles [272].

\subsection{Our work: Near field interference for unidirectional excitation}

In our work [PAPER H] we propose a novel application of near-field interference used for the unidirectional excitation of, not only plasmonic modes, but any other kind of photonic mode propagating in a waveguide. We achieve this by using a nearby circularly or elliptically polarized dipole.

A significant amount of research has been dedicated toward achieving a compact device that can collect light and efficiently excite surface plasmons in a prescribed 

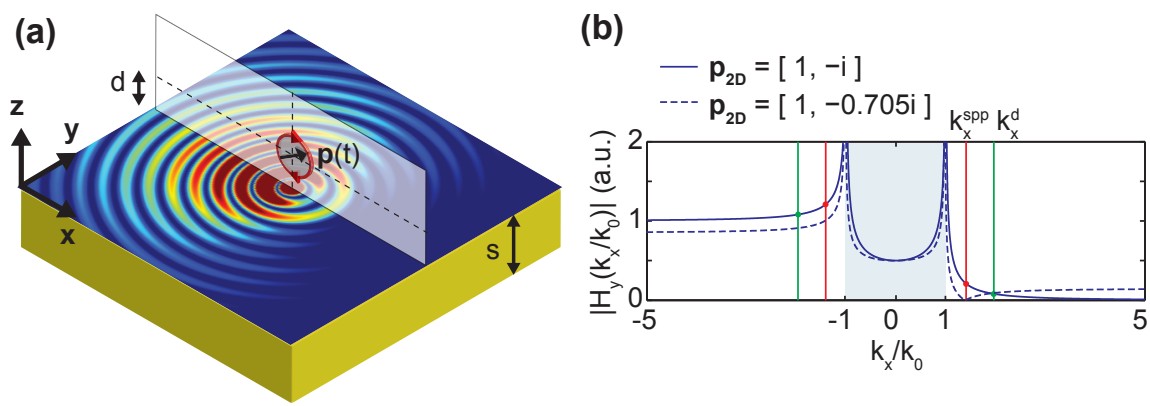

Figure 5.3: (a) Directional excitation of SPPs by a circularly polarized dipole. (b) Spatial-frequency decomposition of a circularly polarized and an elliptically polarized dipole designed to optimize the unidirectionality of SPPs.

direction. Examples include a backside illuminated slit near a surface Bragg grating [273], the use of asymmetric slanted gratings [274] and chirped gratings [275], tilted angle illumination of slits and gratings [276, 277], or the illumination of nearby compact nanoantennas [278]. All of the above approaches rely on the careful selection of the wavelength and geometrical parameters of the structure, and the direction of excitation is determined by an asymmetry of the structure and/or the incident light direction. Our proposed near field dipolar interference provides a fundamentally different approach to unidirectional guided wave excitation with broadband (nonresonant) characteristics and the opportunity to achieve polarization-tuneable directionality.

It is well known (see section 1.4) that a dipole close to a waveguide will excite guided modes thanks to the high spatial frequency components of the near field of the dipole [46]. Typically the excitation of the modes happens in all directions (see Fig. 1.9). In our work [PAPER H], however, we exploit the vectorial nature of the dipole polarization and of the guided mode in order to achieve near field vectorial interference to excite a propagating mode only in one direction [see Fig. 5.3(a)]. This happens independently of the nature of the nearby waveguide, which can be photonic or plasmonic, making the result of interest to the photonic, plasmonic and metamaterials community.

Our proposal requires in general the use of an elliptically polarized dipole, but we show that a circularly polarized dipole is generally enough to achieve a good ratio of unidirectionality. The rotation direction of the dipolar moment determines the direction of guided mode excitation. This is in striking contrast with the far field propagating components, which — as expected due to symmetry - are omnidirectional when the circularly polarized dipole is radiating in free space. There are several ways in which we can explain this counterintuitive phenomenon:

- Vectorial excitation of longitudinal and transverse fields: A simple understanding of the phenomenon can be described as follows: at the point immedi- 
ately below the dipole, the transverse field of the guided mode is driven by the component of the dipole perpendicular to the waveguide, while the longitudinal field is excited by the parallel component of the dipole. The longitudinal and transverse fields of the guided modes have different relative phases depending on the direction of propagation. Thanks to the vector nature of the polarization of the dipole, its parallel and perpendicular components can be judiciously designed with a given amplitude and phase relation between them, such that the interference between them result in the excitation of the nearby guided mode only in a given propagation direction.

- Spatial-frequency decomposition of the dipole: In [PAPER H] we detail a formal demonstration of the effect using the rigorous spatial-frequency Fourier decomposition (see eqs. (2.3) and (2.4)) into plane waves - formally equivalent to the Green function method - of the dipolar fields. We show that a circularly polarized dipole shows vector near field interference between its horizontal and vertical components, resulting in a destructive interference of the evanescent components with a certain sign in the wavevector, while exhibiting constructive interference for the other sign, as shown in Fig. 5.3(b). This constitutes a direct justification of the unidirectional excitation of modes in a nearby waveguide. The plane wave transfer matrix method can be used to analytically obtain the fields in waveguides near a circularly polarized dipole, as shown in Fig. 5.4, confirming the unidirectional excitation of both photonic and plasmonic modes.

- Phase-matching: Probably the most intuitive explanation for the phenomenon is to observe the phase of the circularly polarized dipole radiating in free space. It can be clearly seen (Fig. 5.4 and Movies S1-S4 in [PAPER H]) that the points of equal phase (e.g. the zeros and maxima of the fields) go round in circles following the direction of rotation of the dipole. When a waveguide is brought close to the dipole, the phase of the fields sweeps the surface of the waveguide in a given direction, therefore exciting the guided mode in that direction.

- Symmetry: Using symmetry arguments is another way to explain the phenomenon: the vertical and horizontal dipole moments show even or odd x-symmetry in their field components, due to the "electric wall" or "magnetic wall" symmetry at $x=0$. While the vertical dipole component excites guided modes to the left and right with equal complex amplitude $A$, the horizontal dipole component excites guided modes to the left and right with a $180^{\circ}$ out of phase complex amplitude $B$ and $-B$. Appropriately choosing the amplitudes and phases of the dipole components such that $A=B$-and applying the superposition principle - gives rise to the unidirectional behavior. The left guided mode is excited with an amplitude $A+B=2 A$ while the right one is excited with an amplitude $A-B=0$. 

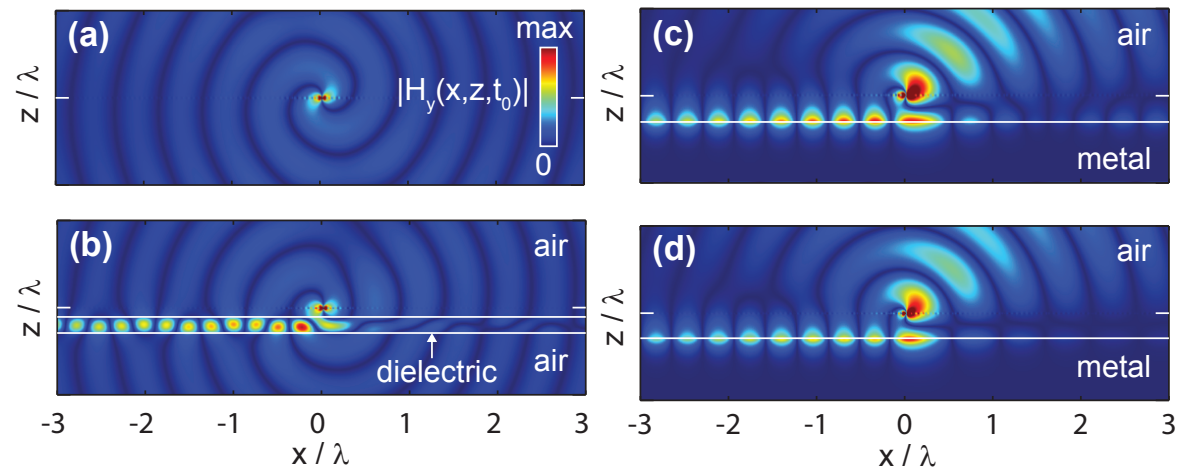

Figure 5.4: (a) 2D equivalent of a circularly polarized dipole $\mathbf{p}_{2 \mathrm{D}}=[1,-i]$ radiating in free space, (b) near a dielectric waveguide, (c) and near a metallic surface supporting SPPs. (d) Elliptically polarized dipole $\mathbf{p}_{2 \mathrm{D}}=[1,-i 0.705]$ designed to optimize the unidirectionality of SPPs near a metallic surface.

To experimentally test the concept, we measured the excitation of SPPs on a metal surface in which a narrow slit was etched. When the slit is illuminated at grazing angle with a circularly polarized beam, as shown in Fig. 5.5(b), the charge distribution in the slit will show a dipolar component ${ }^{2}$ which can be associated with the fields originated by an effective "two-dimensional" dipole with elliptical polarization. Modelling nanostructures such as particles or slits with a point dipole is a typical approach in nanophotonics. The experimental setup is shown in Fig. 5.5(a) and can be described as follows: laser light is polarized circularly by using a polarizer and a $\lambda / 4$ plate, and shone at nearly grazing angle into the slit. The excited SPPs in the metal surface leak into the substrate, and are collected by an oil-inmersion objective. After spatially filtering the collected light to select that leaking out of the smooth metal surface near the slit, a series of lenses are used to obtain the Fourier-space-image of the collected light, which is recorded in a CCD camera and shown in the inset of Fig. 5.5(a). The experimental results are shown in Fig. 5.5(d), where switchable directional excitation of SPPs, as expected from the theory, is observed. Further details of the experiment, together with fabrication procedures and additional results including measurements of a grating, are given in [PAPER H] and its supplementary information.

\footnotetext{
${ }^{2}$ The dipolar component typically dominates over quadrupole and higher multipoles, which decay faster
} 
(a)

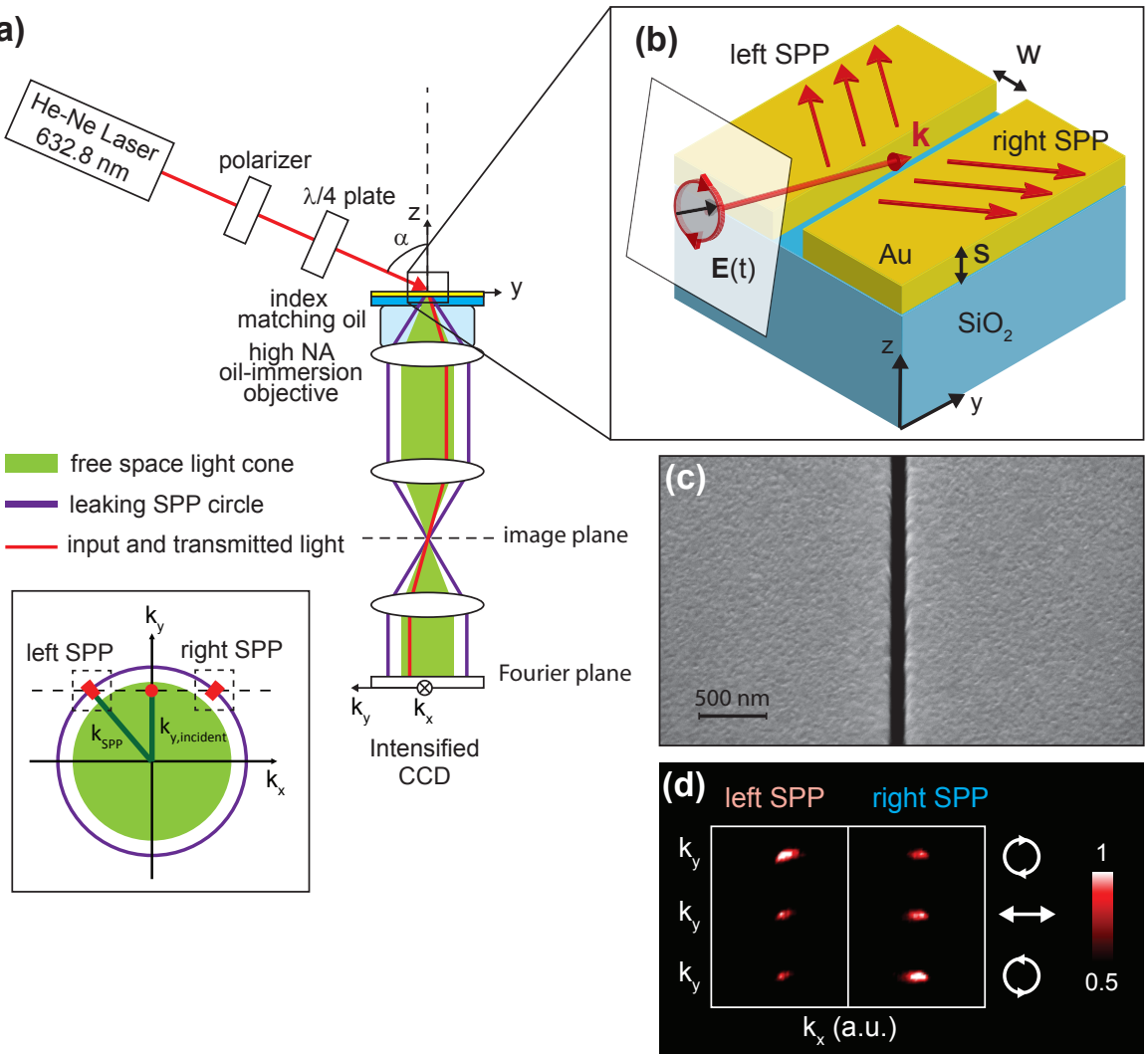

Figure 5.5: Experimental demonstration of the concept. (a) Experimental setup: (b) Illumination of the slit and SPP excitation. (c) Scanning electron micrograph of the fabricated slit (d) Left and right SPP received light spots at the CCD for different polarizations of the input beam, showing the directional behavior under circular polarization. 


\subsection{PAPER G}

Phys. Rev. B 85, 115421 (2012)

\section{Avoiding metallic walls: Use of modal superposition in plasmonic waveguides to reduce propagation loss}

F. J. Rodríguez-Fortuño and N. Engheta 


\title{
Avoiding metallic walls: Use of modal superposition in plasmonic waveguides to reduce propagation loss
}

\author{
Francisco J. Rodríguez-Fortuño ${ }^{1,2}$ and Nader Engheta ${ }^{1}$ \\ ${ }^{1}$ Department of Electrical and Systems Engineering, University of Pennsylvania, Philadelphia, \\ Pennsylvania 19104, USA \\ ${ }^{2}$ Nanophotonics Technology Center, Universidad Politécnica de Valencia, 46022 Valencia, Spain
}

\begin{abstract}
We theoretically explore the possibility of reducing the propagation loss in a metal-insulator-metal (MIM) waveguide, using mode combinations to achieve wall-avoiding field distributions along a certain propagation length. We present analytical results for several waveguides showing notable loss reduction, and we discuss the tradeoffs between low loss and high confinement present in this technique.
\end{abstract}

\section{INTRODUCTION}

The use of plasmonic waveguides to guide electromagnetic energy enables high field confinement ${ }^{1-6}$; however this comes at the expense of a high dissipation due to the ohmic losses in the metal ${ }^{7-9}$. The reduction of the material and propagation losses is a topic of great interest in the plasmonics and metamaterials communities ${ }^{10-17}$. In this Letter, we explore a method for such loss reduction, based on multi-mode interference, which is a known technique $^{18}$ in the field of nanophotonics. In particular, we were inspired by the approach utilized by Popovic et al. for reducing losses in arrays of silicon waveguide crossings ${ }^{19,20}$. In their work, they use a low-loss Bloch wave combination that is judiciously designed to periodically avoid the walls of the silicon waveguide at the crossings. The technique is useful for single crossings as well ${ }^{21-23}$. In the present work, we propose an analogous concept, but for the metal-insulator-metal (MIM) plasmonic waveguides. Multimode behavior in plasmonic waveguides has been observed ${ }^{24}$ and studied for useful devices ${ }^{25,26}$, but not for the purpose we explore here. In this article, we propose the use of a linear combination of modes in a plasmonic waveguide in order to achieve cancellation or reduction of the fields within the metallic walls and thus reduce the power dissipation due to material losses in the transmission of energy from a point $\mathrm{A}$ to a point $\mathrm{B}$ along such a plasmonic waveguide. The losses of the combination of modes are not simply the sum of the losses of each individual mode, since ohmic dissipation depends on the square of the currents in the metal and thus does not behave linearly. We aim to find the proper conditions under which we can minimize such power dissipation for sending electromagnetic power from point $A$ to point $B$ along this waveguide.

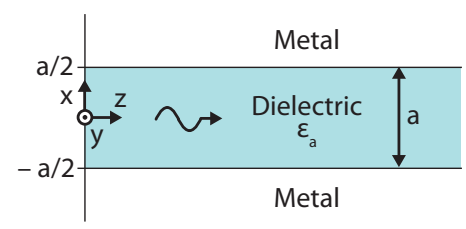

FIG. 1 (color online). Geometry of the metal-insulator-metal (MIM) waveguide 
To illustrate the concept intuitively, we first start by considering a simple, finiteconductivity, parallel plate waveguide, with simple analytical expressions, and then we will consider a realistic MIM waveguide at optical frequencies. Fig. 1 shows the geometry of interest.

\section{FINITE-CONDUCTIVITY PARALLEL-PLATE WAVEGUIDE}

To tackle our simplified waveguide problem, we use a perturbation method: first we calculate the fields inside the paralle-plate waveguide assuming lossless perfect electric conductor (PEC) walls: this gives us a set of well-known orthogonal modes (TEM, TMi and $T E_{i}$, where the integer $i$ refers to the number of half-wavelengths along the width of the waveguide) which we can combine linearly, and only then using the perturbation method we estimate the losses by calculating the currents produced by the total fields in the metallic walls. The electric current in the metallic walls is given by $\mathbf{J}_{s}=\hat{\mathbf{n}} \times \mathbf{H}$, where $\hat{\mathbf{n}}$ is the unit outward vector normal to the walls To achieve the cancellation of $\mathbf{H}$, we must combine modes whose magnetic fields are not orthogonal to each other (i.e. we do not combine a TMi with a $T E_{i}$ mode). Let us restrict ourselves, for example, to the simple combination of only two modes, a TEM and a TMi mode, such that $\mathbf{H}=H_{y} \hat{\mathbf{y}}$. If one adds the field expressions for both modes and then computes the power loss at the conducting walls as $P_{L C}(z)=\left(R_{s} / 2\right)\left(\left|\mathbf{J}_{s}\right|_{x=-a / 2}^{2}+\left|\mathbf{J}_{s}\right|_{x=a / 2}^{2}\right)$, where $R_{s}=\left(\omega \mu(2 \sigma)^{-1}\right)^{1 / 2}$ is the surface resistance of the conductor with conductivity $\sigma, \omega$ is the angular frequency, and $\left|\mathbf{J}_{\mathbf{s}}\right|_{x=x_{0}-2}^{2}=\left|H_{y}\right|_{x=x_{0}}^{2}$, one arrives at the following expression for the losses in the conductor $P_{L C}\left(W \cdot m^{2}\right)$ when $i$ is an even integer (so that both modes TEM and TMi have the same $\mathrm{H}_{\mathrm{y}}$ parity):

$$
P_{L C}(z)=P_{L C T E M}+P_{L C T M i}+2 \sqrt{P_{L C T E M} \cdot P_{L C T M i}} \cos (\Delta k \cdot z)
$$

where $P_{L C T E M}=\left(2 R_{S} / a Z_{\text {TEM }}\right) P_{\text {TEM }}^{+}$and $P_{L C T M i}=\left(4 R_{S} / a Z_{T M i}\right) P_{T M i}^{+}$are the losses for the TEM and $T M_{i}$ modes individually, $Z_{\text {TEM }}=(\mu / \varepsilon)^{1 / 2}$ and $Z_{T M i}=\left(k_{z i} / \omega \varepsilon\right)^{1 / 2}$ are the impedances of these modes, $P_{T E M}^{+}\left(W \cdot m^{-1}\right)$ and $P_{T M i}^{+}\left(W \cdot m^{-1}\right)$ are the powers carried by the TEM and TMi modes, respectively, $P_{T O T}^{+}=P_{T E M}^{+}+P_{T M i}^{+}$and $\Delta k=k_{\text {zTEM }}-k_{\text {zTMi }}=k_{0}\left[1-\left(1-\left(f_{\text {cTMi }} / f\right)^{2}\right)\right]^{1 / 2}$. We note that the average power loss of this mode combination is equal to the sum of the power loss of the two modes propagating individually, but locally the power loss varies with $z$. Since we can make $2 \sqrt{P_{\text {LCTEM }} \cdot P_{\text {LCTMi }}}$ equal to (but never greater than) $P_{\text {LCTEM }}+P_{\text {LCTMi }}$, the value of power dissipation can become identically zero at a certain point $z$ along the waveguide if we fulfill the condition $P_{\text {LCTMi }}=P_{\text {LCTEM }}$, which can also be written as

$$
P_{T M i}^{+} / P_{T E M}^{+}=\frac{1}{2} \frac{Z_{T M i}}{Z_{T E M}}=\frac{1}{2} \sqrt{1-\left(f_{\text {cTMi }} / f\right)^{2}} .
$$

This condition achieves something interesting: Exactly at zo, the free electrons in the metallic walls are not experiencing any forces at all from the electromagnetic fields which are being guided between the two walls, and thus locally this guiding is not generating any ohmic losses. Figure 2 shows the field distribution and power flow of such combination of modes TEM and $\mathrm{TM}_{2}$ under the condition (2), together with the power loss at the conducting walls $P_{L C}$. The power flow varies from being confined at the center of the waveguide at $z_{0}$, to flowing near the walls at $z_{0} \pm L_{2 \pi} / 2$. The spatial period of this periodic behavior is given by $L_{2 \pi}=2 \pi /\left(k_{\text {zTEM }}-k_{\text {zTM } 2}\right)$. 


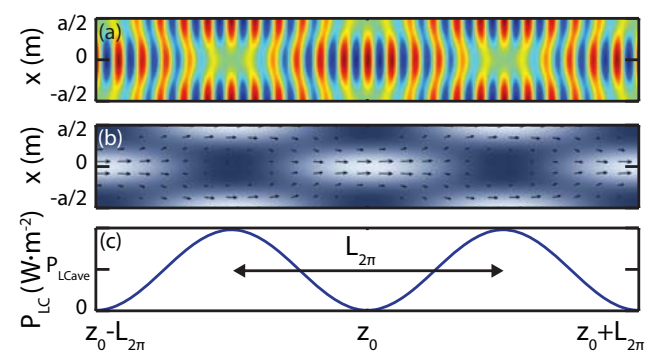

FIG. 2 (color online). (a) $\mathrm{H}_{\mathrm{y}}$ field of the linear combination of TEM and $\mathrm{TM}_{2}$ modes under condition (2) assuming PEC walls. (b) Poynting vector. (c) Dissipated power if the metal walls have a finite conductivity

Equation (1) can be proved to be valid for any other combination of two TMi modes given that they have the same $H_{y}$ parity. On the other hand, if the combined modes have opposite $H_{y}$ parity, then we simply obtain $P_{L C}(\mathrm{z})=P_{\text {LCTMi }}+P_{\text {LCTMj }}$. In that situation, the power dissipation "bounces" from one wall to another, but the sum for both walls is constant at any point (e.g. when combining TEM and TM1).

Now imagine that we want to transfer energy through this waveguide. We can make use of the drastically reduced power dissipation region around $z_{0}$ to transfer energy from A $\left(z_{0}-L / 2\right)$ to $\mathrm{B}\left(z_{0}+L / 2\right)$ over a length $L$ that must be small compared to $L_{2 \pi}$ (the beat length of the two modes). To do this, we should excite point $\mathrm{A}$ with the mode profile that corresponds to the appropriate mode combination at that point. The effective dissipated power by the TEM + TMi (even $i$ ) mode combination, under condition (2), for a length $L$ around $z_{0}$ can be defined and calculated as

$$
P_{L C e f f}(L) \equiv \frac{1}{L} \int_{z_{0}-L / 2}^{z_{0}+L / 2} P_{L C}(z) d z=\left(P_{L C T E M}+P_{L C T M}\right) \cdot\left(1-\operatorname{sinc}_{\pi}\left(\frac{\Delta k L}{2 \pi}\right)\right),
$$

where $\operatorname{sinc}_{\pi}(x)=\sin (\pi x) / \pi x$ and we can rewrite $\Delta k L / 2 \pi=L / L_{2 \pi}=(L / \lambda)\left(1-\sqrt{1-\left(f_{\text {стмі }} / f\right)^{2}}\right)$ where $\lambda=\left(c_{0} / f\right) \varepsilon_{r a}^{-1 / 2}$. The first term is the sum of the losses of the two modes individually, but clearly the second term can be very small if $L$ is sufficiently small compared to $L_{2 \pi}$, so that reduced losses are achievable. To obtain a fair insight into the improvement offered by this technique, we should compare the dissipated power $P_{\text {LCeff }}$ under condition (2) with the smallest achievable dissipated power at the conductor using a single mode in the parallel plate waveguide, which is by using the TEM mode to carry all the power $P_{\text {TOT }}^{+}$by itself. Such comparison yields the following loss ratio $\left(L R=P_{L C \text { Ceff }}^{\text {Condion (2) }} / P_{L C T E M}^{P_{\text {TEM }}^{+}=P_{\text {TOT }}^{+}}\right)$

$$
L R=\left(\frac{2}{1+(1 / 2) \sqrt{1-\left(f_{c T M i} / f\right)^{2}}}\right)\left(1-\operatorname{sinc}_{\pi}\left(\frac{L}{\lambda}\left(1-\sqrt{1-\left(f_{\text {cTMi }} / f\right)^{2}}\right)\right)\right) .
$$

Using this simple expression we can plot normalized graphs showing the loss ratio (LR) as a function of propagation length $L$ and width of the waveguide $a$ both normalized to $\lambda$, as 
seen in Fig. 3. Notice that this graph is independent of the frequency and of the metal conductivity, so it can be regarded as a general result.

We see that significant loss reduction over a propagation distance of many wavelengths may be achieved if we work at high values of $\left(f / f_{\text {сTMi }}\right)$. For example: by combining TEM and $\mathrm{TM}_{2}$ in a waveguide with $a=10 \lambda$ we can achieve a loss ratio of less than 0.1 (i.e. more than $90 \%$ reduced loss) from point A to B separated by a length $L=40 \lambda$. These results can readily be applied at microwave or terahertz frequencies. As we note, it is desirable for $f / f_{\text {стM }}$ to be high (which translates into an electrically wide waveguide), since this means that the $T M_{i}$ mode is well above its cutoff frequency, and therefore has a very similar propagation constant to that of the TEM mode, achieving a long beat length $L 2 \pi$ and therefore a long region of small losses around $z$. We will see that this tradeoff between small waveguide width and low losses is present throughout all our results.

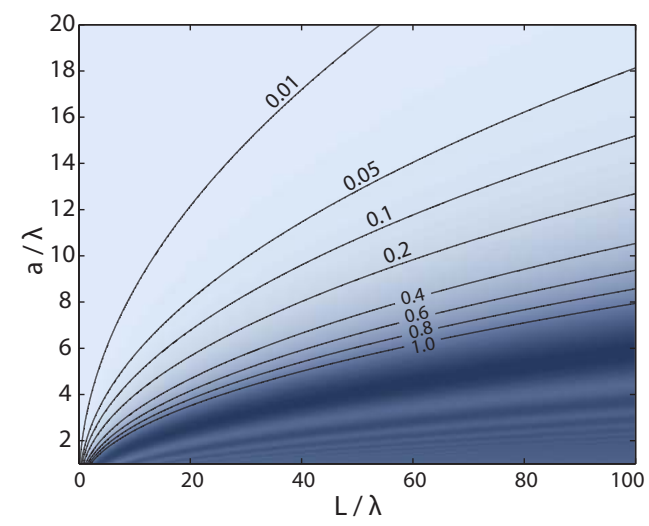

FIG. 3 (color online). Loss ratio (LR) as a function of propagation length $L / \lambda$ and waveguide width $a / \lambda$ for a combination of TEM and TM2 modes. In general, for a TMi mode, the vertical axis can be changed to $f / f_{\text {cTMi }}=(i / 2)(a / \lambda)$

\section{MIM PLASMONIC WAVEGUIDE}

We now extend this study to the case of MIM plasmonic waveguide with the aim of working at optical (infrared and visible) frequencies. In this case the electromagnetic fields penetrate into the metal, and the currents in the metal are given by $\mathbf{J}=\sigma_{m} \mathbf{E}$, where $\sigma_{m}$ is the conductivity, so our aim is to achieve cancellation of the $\mathbf{E}$ field inside the metal using a linear combination of modes. The same symmetry considerations still apply. We will consider TM electromagnetic modes in a MIM plasmonic waveguide, whose field distributions and dispersion relations are known ${ }^{8}$. We use the following nomenclature: each mode $i$ will have a given propagation constant $k_{z i}$ at a given frequency $\omega$. The variation of fields along the transverse $x$ direction will be of the type $\exp \left( \pm \alpha_{m i} x\right)$ and $\exp \left( \pm \alpha_{a i} x\right)$ in the metal or in the dielectric region, respectively, where $\alpha_{m i, a i}^{2}=k_{z i}^{2}-\omega^{2} \mu \varepsilon_{m, d}$. The mode amplitude will be proportional to a scaling factor $M_{i}=H_{y}(x=a / 2, z=0)$. For the MIM geometry, only two modes exist below the dielectric light cone corresponding to the even 
and odd plasmonic modes (real $\alpha_{a i}$ ) while an infinite number of even and odd modes exist above the dielectric light cone, which show trigonometric variation in the $x$ direction (imaginary $\alpha_{a i}$ ) which we refer to as dielectric slab modes. In order to cancel out or reduce the fields in the metal, we can choose to combine any two modes as long as they have the same parity. Again we use a perturbation method: we first calculate the two modes at a given frequency assuming lossless plasmonic metal (considering only a real part in the permittivity of metal $\varepsilon_{m}^{\prime}$ ). Then we add the two mode's fields and estimate the losses of the total fields by taking into account the imaginary part of the metal permittivity $\varepsilon_{m}^{\prime \prime}$. This method is valid only as long as the imaginary part of the permittivity does not substantially alter the mode's field profiles (i.e. that the perturbed lossy modal shapes are sufficiently similar to those of the lossless modes). To check this, we calculated that, in all the single modes used as examples in this paper, the estimated attenuation coefficient using the perturbation method described was within a $0.1 \%$ error of the exact attenuation coefficient obtained from an exact solution of the modes with a complex permittivity metal. For all our calculations, we take the values from Johnson and Christy $\varepsilon_{m}=\varepsilon_{m}^{\prime}+i \varepsilon_{m}^{\prime \prime}$ for silver. ${ }^{27}$ According to the perturbation method, the losses in the metal are evaluated using

$$
P_{L M}=\frac{\omega \varepsilon_{m}^{\prime \prime}}{2}\left(\int_{a / 2}^{\infty}|\mathbf{E}|^{2} d x+\int_{-a / 2}^{-\infty}|\mathbf{E}|^{2} d x\right)
$$

which for a single mode $i$ (with field amplitude $M_{i}$ and propagation constant $k_{z i}$ ) can be calculated by substituting the fields of a single mode into (5) resulting in

$$
P_{L M i}=\frac{\omega \varepsilon_{m}^{\prime \prime}}{2 \alpha_{m i}}\left(\frac{\left|M_{i}\right|}{\omega \varepsilon_{m}^{\prime}}\right)^{2}\left(k_{z i}^{2}+\alpha_{m i}^{2}\right) .
$$

When linearly combining two modes $i=1$ and 2, we first add the fields for the modes and then we apply equation (5), so after some mathematical manipulation we arrive at the following expression for the power dissipation of the mode combination, which is the main result of our work:

$$
P_{L M}(z)=P_{L M 1}+P_{L M 2}+2 \sqrt{P_{L M 1} \cdot P_{L M 2}} \cdot \eta_{\alpha} \cdot \eta_{x z} \cdot \cos (\Delta k z+\Delta \phi)
$$

where $P_{L M 1}$ and $P_{L M 2}$ are given by (6), $\eta_{\alpha}=2\left(\alpha_{m 1} \alpha_{m 2}\right)^{1 / 2}\left(\alpha_{m 1}+\alpha_{m 2}\right)^{-1} \in[0,1]$, $\eta_{x z}=\left(\alpha_{m 1} \alpha_{m 2}+k_{z 1} k_{z 2}\right)\left(\alpha_{m 1}^{2} \alpha_{m 2}^{2}+k_{z 1}^{2} k_{z 2}^{2}+\alpha_{m 1}^{2} k_{z 2}^{2}+\alpha_{m 2}^{2} k_{z 1}^{2}\right)^{-1 / 2} \in[0,1], \quad \Delta k=k_{z 2}-k_{z 1}$, and $\Delta \phi=\angle M_{2}-\angle M_{1}$. Equation (7) can be shown to be valid for any combination of two TM modes in this geometry with the same parity. It is similar to the near perfect conductor case (1), but now two terms $\eta_{x z}$ and $\eta_{\alpha}$ prevent $P_{L M}\left(z_{0}\right)$ from becoming identically zero. The coefficient $\eta_{x z}$ arises due to the fact that there are two components of the currents in the metal, $x$ and $z$, whose ratio is different for the two different modes, so having only one free parameter to tune (the relative power between the modes) we cannot exactly cancel both components simultaneously. The coefficient $\eta_{\alpha}$ takes into account the fact that the rate of evanescent decay of fields into the metal is slightly different for both modes so the field cancellation cannot be identically zero for all the region $|x|>a / 2$. In practice, however, both coefficients are very close to 1 . Looking at (7) we can deduce that optimization of losses at $z_{0}$ takes place when $P_{L M 1}=P_{L M 2}$ which, in accordance to (6), happens when 
$\left|M_{2}\right| /\left|M_{1}\right|=\sqrt{\alpha_{m 2} \alpha_{m 1}^{-1}\left(k_{z 1}^{2}+\alpha_{m 1}^{2}\right)\left(k_{z 2}^{2}+\alpha_{m 2}^{2}\right)^{-1}}$. This simple and analytically exact condition guarantees minimum power dissipation at $z$. We can force this condition by appropriately tuning the relative power carried by each mode. The experimental excitation of the two modes with the appropriate amplitudes and phases may prove challenging. However, the mode profile that has to be excited at point $\mathrm{A}$ is concentrated near the center of the waveguide, so exciting the MIM waveguide with a Gaussian beam or a single-mode dielectric waveguide, similar to what is done in Refs. [3-6], may be possible ways to experimentally access the $z_{0}$ region.

TABLE I. Individual mode and mode combination parameters for the two examples A and $B$.

\begin{tabular}{|c|c|c|}
\hline & A (narrow waveguide) & B (wide waveguide) \\
\hline$a(\mu \mathrm{m})$ & 0.8 & 6.0 \\
$\lambda_{0}(\mu \mathrm{m})$ & 0.6 & 0.6 \\
\hline Mode 1 & even plasmonic mode & $1^{\text {st }}$ even dielectric slab mode \\
$k_{21}\left(\mathrm{rad} \cdot \mu \mathrm{m}^{-1}\right)$ & 16.3 & 15.18 \\
$\alpha_{21}\left(\mathrm{~dB} \cdot \mu \mathrm{m}^{-1}\right)$ & 0.275 & $0.159 \cdot 10^{-3}$ \\
\hline Mode 2 & $1^{\text {st }}$ even dielectric slab mode & $2^{\text {nd }}$ even dielectric slab mode \\
$k_{22}\left(\mathrm{rad} \cdot \mu \mathrm{m}^{-1}\right)$ & 14.0 & 15.10 \\
$\alpha_{22}\left(\mathrm{~dB} \cdot \mu \mathrm{m}^{-1}\right)$ & 0.0867 & $1.33 \cdot 10^{-3}$ \\
\hline Mode combination & & \\
$P_{\operatorname{mode2}}^{+} / P_{\operatorname{mode1}}^{+}$ & 3.175 & 0.119 \\
$\eta_{x z}$ & 0.9991 & 1.0000 \\
$\eta_{\alpha}$ & 1.0000 & 1.0000 \\
$\Delta k_{z}\left(\mathrm{rad} \cdot \mu \mathrm{m}^{-1}\right)$ & 3.3 & 0.08 \\
$\alpha_{\text {ave }}\left(\mathrm{dB} \cdot \mu \mathrm{m}^{-1}\right)$ & 0.132 & $0.284 \cdot 10^{-3}$ \\
$L_{2 \pi}(\mu \mathrm{m})$ & 1.34 & 77.4 \\
$L_{\text {max }}(\mu \mathrm{m})$ & 0.65 & 50.0 \\
\hline
\end{tabular}

We will consider two examples demonstrating the features of the proposed technique, both of which work in the visible spectrum $f=500 \mathrm{THz} \quad\left(\lambda_{0} \approx 0.6 \mu \mathrm{m}\right)$ where $\varepsilon_{m}(500 T H z)=-16.04+0.4327 i$ and both use silica as the dielectric $\left(\varepsilon_{a}=1.45^{2}\right)$. To gain some insight and allow easy comparisons, we first calculate a local attenuation coefficient $\alpha_{z}(z)=P_{L M}(z) / 2 P_{T O T}^{+}$which for individual modes is a constant value and represents the power attenuation $P^{+}(z) \propto \exp \left(-\alpha_{z} z\right)$, but which for the combination of the two modes has a sinusoidal variation in $z$ with period $L_{2 \pi}$ reaching a minimum of $\alpha_{\text {ave }}\left(1-\eta_{x z} \eta_{\alpha}\right) \approx 0$ at $z_{0}$ and a maximum of $\alpha_{\text {ave }}\left(1+\eta_{x z} \eta_{\alpha}\right) \approx 2 \alpha_{\text {ave }}$ at $z_{0} \pm L_{2 \pi} / 2$, where $\alpha_{\text {ave }}$ is the average value of the attenuation coefficient of the two modes, weighed by their relative power. Then, for a given mode combination and a given propagation length $L$, we define an effective attenuation coefficient $\alpha_{\text {eff }}(L)=\frac{1}{L} \int_{z_{0}-L / 2}^{z_{0}+L / 2} \alpha_{z}(z) d z$, which if $\eta_{x z}$ and $\eta_{\alpha}$ are close to one, can be approximated by $\alpha_{\text {eff }}(L) \approx \alpha_{\text {ave }} \cdot\left(1-\operatorname{sinc}_{\pi}\left(L / L_{2 \pi}\right)\right)$. In addition, it is interesting to 
compute the maximum propagation length $L_{\max }$ for which the use of the mode combination technique shows an improvement in terms of the total loss over any of the two constituent modes individually, that is, $L \leq L_{\max } \Rightarrow \alpha_{\text {eff }}(L) \leq \min \left(\alpha_{z 1}, \alpha_{z 2}\right)$. All the relevant parameters for the two examples are summarized in Table I.
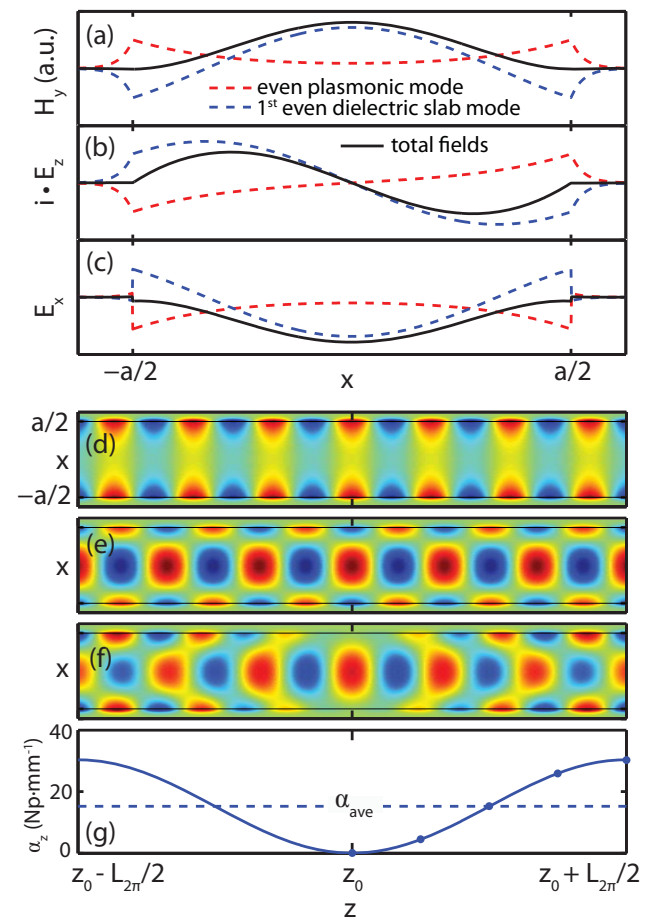

FIG. 4 (color online). Electromagnetic field components (a) $\mathrm{H}_{\mathrm{y}}(\mathrm{x})(\mathrm{b}) \mathrm{i} \mathrm{E}_{\mathrm{z}}(\mathrm{x})$ and (c) $\mathrm{Ex}_{\mathrm{x}}(\mathrm{x})$ at $z=z_{0}$ of both individual modes and the linear combination of both under the condition for minimized losses at $z=z_{0} \cdot H_{y}(x, z)$ field distribution for (d) the even plasmonic mode, (e) the $1^{\text {st }}$ even dielectric slab mode and (f) the linear combination of both. (g) Local attenuation coefficient in the metal for the linear combination of modes. The continuous line represents the analytical calculation of Eq. (7) while the dots represent the loss numerically calculated from the field distribution.

The first example, whose fields are shown in Fig. 4, is a narrow MIM waveguide where we adequately combine the even plasmonic mode with the first even dielectric slab mode to achieve reduction of the fields in the metal around $z$. The cancellation of fields inside the metal at $z_{0}$ is almost perfect. Unfortunately, in this case $L_{\max }$ is not a very long distance relative to the wavelength due to the high $\Delta k_{z}$. Notice that we could use mode engineering using multilayered dielectrics between the two metals to further reduce $\Delta k_{z}$ and therefore increase $L_{\max }$.

Alternatively we can reduce $\Delta k_{z}$ by using more closely related modes. This is what we do in the second example, where we combine two dielectric slab modes on a wide waveguide so 
that their propagation constants are close to each other and to that of the dielectric, at the expense of lowering the confinement. This achieves a relatively large $L_{\max }=50 \mu \mathrm{m}$. So if we want to transfer energy on such MIM waveguide for a distance $|L|<50 \mu m$, the use of the appropriate mode combination as shown will reduce the total loss with respect to using a single mode. In this case the improvement can be very noticeable for smaller distances $L$, for example, if $L=10 \mu \mathrm{m}$, then the effective attenuation constant is as low as $\alpha_{\text {eff }}(10 \mu \mathrm{m})=7.73 \mathrm{~dB} \cdot \mathrm{m}^{-1}$, considerably smaller than the $159 \mathrm{~dB} \cdot \mathrm{m}^{-1}$ of the lowest loss dielectric slab mode.

\section{CONCLUSIONS}

In conclusion, using the linear combination of two modes in a MIM waveguide we have found the condition under which the losses of the mode combination are minimized to almost zero at a given point in the waveguide. Transmission of energy for a length $\mathrm{L}$ in the neighborhood of that point shows very low total power dissipation. Our study was performed both for good conductors, valid at microwave and terahertz frequencies, and for Drude type metals at optical frequencies, yielding very similar conclusions. The reduction of losses comes however at the cost of reducing the high confinement typical of plasmonic modes. Since we require modes with geometrical variation inside the dielectric, the width of the waveguide must be comparable to the wavelength. Under such condition, one may conceivably use a dielectric waveguide altogether showing zero metal dissipation, however, the presence of metal can be useful for other purposes such as electrodes in electro-optical modulator. Therefore, our present work is useful in devices where the metals are unavoidable or desirable.

\section{Acknowledgements}

F.J. Rodríguez-Fortuño acknowledges financial support from grant FPI of GV and the Spanish MICINN under contracts CONSOLIDER EMET CSD2008-00066 and TEC2011-28664C02-02. This work is supported in part by the Office of Naval Research (ONR)

Multidisciplinary University Research Initiative (MURI) grant number N00014-10-1-0942.

\section{References}

${ }^{1}$ W.L. Barnes, A. Dereux, and T.W. Ebbesen, Nature 424, 824-830 (2003).

2 J. a Schuller, E.S. Barnard, W. Cai, Y.C. Jun, J.S. White, and M.L. Brongersma, Nature Materials 9, 193-204 (2010).

${ }^{3}$ E. Economou, Physical Review 182, 539-554 (1969).

${ }^{4}$ C. Pfeiffer, E. Economou, and K. Ngai, Physical Review B 10, 3038-3051 (1974).

${ }^{5}$ J. Takahara, S. Yamagishi, H. Taki, A. Morimoto, and T. Kobayashi, Optics Letters 22, 475 (1997).

${ }^{6}$ S.A. Maier and H.A. Atwater, Journal of Applied Physics 98, 011101 (2005).

${ }^{7}$ P. Berini, Optics Express 14, 13030 (2006).

${ }^{8}$ S.A. Maier, Plasmonics: Fundamentals and Applications (Springer;, 2010), p. 248.

${ }^{9}$ E. Ozbay, Science (New York, N.Y.) 311, 189-93 (2006). 
10 A. Boltasseva and H.A. Atwater, Science (New York, N.Y.) 331, 290-1 (2011).

${ }^{11}$ D.A. Bobb, G. Zhu, M. Mayy, A.V. Gavrilenko, P. Mead, V.I. Gavrilenko, and M.A. Noginov, Applied Physics Letters 95, 151102 (2009).

${ }^{12}$ R.F. Oulton, V.J. Sorger, D.A. Genov, D.F.P. Pile, and X. Zhang, Nature Photonics 2, 496500 (2008).

${ }^{13}$ J.T. Kim, J.J. Ju, S. Park, M.-su Kim, S.K. Park, and S.-Y. Shin, Optics Express 18, 2808 (2010).

${ }^{14}$ L. Chen, J. Shakya, and M. Lipson, Optics Letters 31, 2133 (2006).

${ }^{15}$ S.A. Maier, P.E. Barclay, T.J. Johnson, M.D. Friedman, and O. Painter, Applied Physics Letters 84, 3990 (2004).

${ }^{16}$ D. Dai and S. He, Optics Express 18, 17958 (2010).

${ }^{17}$ M. Wu, Z. Han, and V. Van, Optics Express 18, 11728 (2010).

${ }^{18}$ L.B. Soldano and E.C.M. Pennings, Journal of Lightwave Technology 13, 615-627 (1995).

${ }^{19}$ M.A. Popovic, University of Colorado Boulder, personal communication

${ }^{20}$ M.A. Popovic, E.P. Ippen, and F.X. Kartner, LEOS 2007 - IEEE Lasers and Electro-Optics Society Annual Meeting Conference Proceedings 56-57 (2007).

${ }^{21}$ H.R. Stuart, Optics Letters 28, 2141-3 (2003).

${ }^{22}$ H. Liu, H. Tam, P. Wai, and E. Pun, Optics Communications 241, 99-104 (2004).

${ }^{23}$ H. Chen and A.W. Poon, IEEE Photonics Technology Letters 18, 2260-2262 (2006).

${ }^{24}$ B. Steinberger, A. Hohenau, H. Ditlbacher, A.L. Stepanov, A. Drezet, F.R. Aussenegg, A. Leitner, and J.R. Krenn, Applied Physics Letters 88, 094104 (2006).

${ }^{25}$ Y.-J. Tsai, A. Degiron, N.M. Jokerst, and D.R. Smith, Optics Express 17, 17471 (2009).

${ }^{26}$ Y. Kou and X. Chen, Optics Express 19, 6042 (2011).

${ }_{27}$ P.B. Johnson and R.W. Christy, Physical Review B 6, 4370-4379 (1972). 


\title{
5.6 PAPER H
}

Science 340, 328 (2013)

\section{Near field interference for the unidirectional excitation of electromagnetic guided modes}

\author{
F. J. Rodríguez-Fortuño, G. Marino, P. Ginzburg, \\ D. O'Connor, A. Martínez, G. A. Wurtz and A. V. Zayats
}

This is the author's version of the work. It is posted here by permission of the AAAS for personal use, not for redistribution. The definitive version was published in Science vol. 340 (19 April 2013), doi: 10.1126/science.1233739 Note: the electronic version of this thesis does not include the article due to the "License to Publish" signed with AAAS. 


\title{
Near field interference for the unidirectional excitation of electromagnetic guided modes
}

\author{
Francisco J. Rodríguez-Fortuño, ${ }^{1,2}$, Giuseppe Marino ${ }^{1}$, Pavel Ginzburg ${ }^{1}$, Daniel O'Connor ${ }^{1}$ \\ Alejandro Martínez ${ }^{2}$, Gregory A. Wurtz ${ }^{1}$ and Anatoly V. Zayats ${ }^{1}$ \\ ${ }^{1}$ Department of Physics, King's College London, Strand, London WC2R 2LS, United Kingdom \\ 2 Nanophotonics Technology Center, Universitat Politècnica de València, Valencia 46022, Spain
}

\begin{abstract}
Wave interference is a fundamental manifestation of the superposition principle with numerous applications. While in conventional optics interference occurs between waves undergoing different phase advances during propagation, we show that the vectorial structure of the near-field of an emitter is essential for controlling its radiation as it interferes with itself on interaction with a mediating object. We demonstrate that the near field interference of a circularly polarized dipole results in the unidirectional excitation of guided electromagnetic modes in the near-field, with no preferred far-field radiation direction. By mimicking the dipole with a single illuminated slit in a gold film, we measured unidirectional surface-plasmon excitation in a spatially symmetric structure. The surface wave direction is switchable with the polarization.
\end{abstract}

Interference is the cornerstone of various phenomena in nature enabling numerous applications. In optics, it is intensively used in microscopy, stellar measurements, spectroscopy, and communication technologies, among many others, and is the basis behind the concepts of reflection, refraction and light bending $(1,2)$. Typically, interference occurs due to the relative phase lag of different propagating waves. On the other hand, nanophotonics -the branch of optics studying the interaction of light with subwavelength nanoscale structures- deals inherently with phenomena that occur via near-field interactions before appreciable phase lags can be accumulated (3). A radiationless form of interference in the near field (4) is behind new exciting applications such as the focusing of evanescent components to achieve subwavelength resolution in imaging (5-8). Near field interference achieved through the full coherent control of the phase and amplitude of excitation light allows asymmetric spatial field localization $(9,10)$ and selection of propagation paths at intersections of waveguides (11).

We demonstrate near field interference by considering a single source of radiation coupled to a mode with a vectorial structure of electromagnetic field. Using an additional degree of freedom provided by the vectorial character of the field, control over the near-field interference can be achieved. We show that an elliptically polarized dipole can produce destructive or constructive interference of different evanescent components in its near field, and as a result, excite electromagnetic modes in neighbouring material structures, such as dielectric and plasmonic waveguides and diffraction gratings, with a controlled directionality of propagation. 

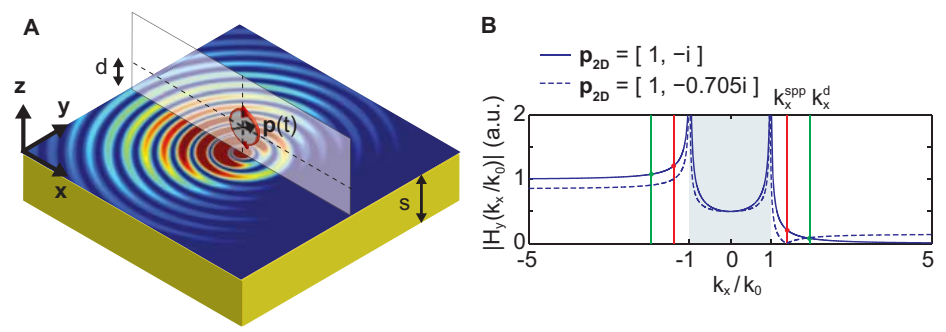

Figure 1. (A) Schematics of the circularly polarized dipole over a planar waveguiding structure (metal-dielectric interface). A cross-section of the calculated magnetic field distribution is overlaid at the surface of a metal $(\varepsilon=-2+0.1 \mathrm{i}, \mathrm{d}=0.3 \lambda)$, showing the asymmetric excitation of SPPs. (B) Spatial frequency spectra of a circularly polarized (continuous line) and elliptically polarized (dashed line) dipole.

Recently, several solutions for the directional excitation of surface plasmon polariton (SPP) waves $(12,13)$ have been proposed, including a backside illuminated slit near a surface Bragg grating (14), the use of asymmetric slanted gratings (15) and chirped gratings (16), tilted angle illumination of slits and gratings $(17,18)$, or the illumination of nearby compact nanoantennas (19). Directional extraction of light from localized emitters with nanoantennas (20) has been demonstrated. All of the above approaches have a resonant nature and rely on the careful selection of the wavelength and geometrical parameters of the structure, with the direction of excitation determined by an asymmetry of the structure and/or the incident light direction. The directional coupling to planar dielectric photonic waveguides is typically achieved with diffraction gratings (21) carefully designed for a specific direction of excitation. Near field dipolar interference provides a fundamentally different approach to unidirectional guided wave excitation with broadband (nonresonant) characteristics and the opportunity to achieve polarization-tuneable directionality.

The phenomenon of unidirectional excitation can be understood by considering a dipole placed at a subwavelength distance $d$ from a waveguide surface, for example a dielectric slab or a single metal-dielectric interface (Fig. 1A). The high spatial frequency components of the dipole radiation allow the excitation of guided modes in the waveguide. The fundamental principle behind the effect of unidirectionality is the selective vectorial excitation of the electrical field of the waveguide mode, depending on the emitted polarization. At the location of the waveguide immediately below the dipole, the vertically oriented component of the dipole is coupled to the transverse field of the electromagnetic mode, while the horizontal component of the dipole drives the longitudinal component. Both components have different amplitude and phase. A properly chosen polarization of the dipole will result in destructive interference in one prescribed direction and a mode propagating in the opposite direction.

Without restriction on the nature of waveguided modes, we consider a rigorous quantitative description based on phase-matching conditions between the source dipole and the structure's modes. It is convenient to decompose the field of the dipole into spatial-frequency components $\mathrm{k}_{\mathrm{x}}$ and $\mathrm{k}_{\mathrm{y}}(3)$. The full three dimensional (3D) treatment is described in (22). Here we discuss a two-dimensional (2D) case in which the dipole moment is defined per unit 
length as $\mathbf{p}_{2 \mathrm{D}}=\left[\mathrm{p}_{\mathrm{x}}, \mathrm{p}_{\mathrm{z}}\right]$, and the decomposition of its magnetic field can be written as $H_{y}(x, z)=\int H_{y}\left(k_{x}, z\right) e^{i k_{x} x} d k_{x}$, where

$$
H_{y}\left(k_{x}, z\right)=\frac{i \omega}{8 \pi^{2}}\left[p_{z} \frac{k_{x}}{k_{z}} \mp p_{x}\right] e^{i k_{z}|z|},
$$

$\mathrm{k}_{\mathrm{z}}=\left(\mathrm{k}_{0}{ }^{2}-\mathrm{k}^{2}\right)^{1 / 2}$ is the wavevector along $\mathrm{z}, \omega$ is the angular frequency, $\mathrm{k}_{0}=\omega / \mathrm{c}$, and the phasor

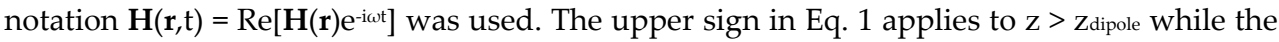
lower sign applies to $\mathrm{z}<$ zdipole, where $\mathrm{zdipole}$ is the dipole coordinate. The $\mathrm{H}_{\mathrm{y}}$ components with $-\mathrm{k}_{0}<\mathrm{k}_{\mathrm{x}}<\mathrm{k}_{0}$ correspond to propagating plane waves forming an angle $\theta=\sin ^{-1}\left(k_{x} / k_{0}\right)$ with the $z$-axis, while the components with $\left|k_{x}\right|>k_{0}$ are evanescent and present significantly only in the near field (for $\mathrm{z}<<\lambda$ ).
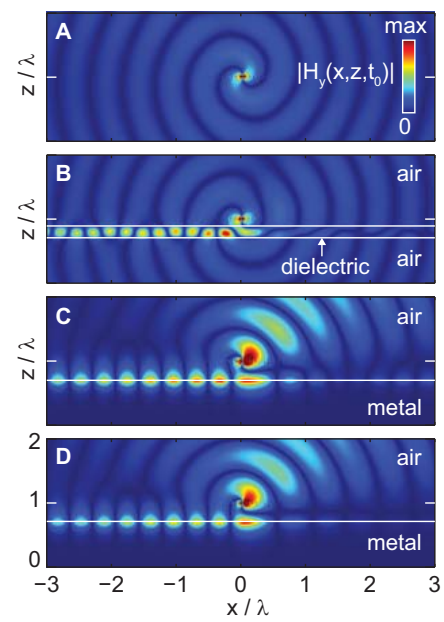

Figure 2. Magnetic field distributions for a $2 \mathrm{D}$ circularly polarized dipole $\mathbf{p}_{2 \mathrm{D}}=[1,-\mathrm{i}](\mathrm{A})$ radiating in free space, $(B)$ exciting a dielectric waveguide mode $(\mathrm{d}=0.1 \lambda, \varepsilon=9+0.1 \mathrm{i}, \mathrm{s}=$ $0.2 \lambda)$, and (C) exciting SPP mode ( $d=0.3 \lambda, \varepsilon=-2+0.1 \mathrm{i}, \mathrm{s}=\infty)$. (D) Same as (C) but with a optimized elliptical dipole $\mathbf{p}_{2 \mathrm{D}}=[1,-0.705 \mathrm{i}]$ to minimize the SPP excitation in the opposite direction. Colour scale is the same for all plots.

The linear superposition of the two orthogonal dipole orientations $p_{x}$ and $p_{z}$ leads to both far-field and near-field interference. The dipole oriented along $\mathbf{z}$ has an odd parity of its spatial frequency spectrum $\mathrm{H}_{\mathrm{y}}\left(\mathrm{k}_{\mathrm{x}}\right)$, while the opposite is true for the $\mathbf{x}$-oriented dipole, which shows an even parity. This suggests that applying the superposition principle to these dipoles could result in a non-symmetric spatial-frequency distribution. The term $\left(k_{x} / k_{z}\right)$ in Eq. (1) is real valued for propagating components and imaginary for evanescent ones. This means that in order to achieve destructive interference $\left[\mathrm{H}_{\mathrm{y}}\left(\mathrm{k}_{\mathrm{x}}\right)=0\right]$ inside the light cone $\left(\mathrm{k}_{\mathrm{x}}<\mathrm{k}_{0}\right), \mathrm{p}_{\mathrm{x}}$ and $\mathrm{p}_{\mathrm{z}}$ should be in phase (linearly polarized). Remarkably, the interference can take place outside the light cone $\left(k_{x}>k_{0}\right)$ when $p_{x}$ and $p_{z}$ are $90^{\circ}$ out of phase. According to Eq. (1), for a circularly polarized dipole with $\mathbf{p}_{2 \mathrm{D}}=p[1,-i] / \sqrt{2}$, the spectral amplitude of the evanescent components with $k_{x}>0$ add up destructively, while for negative $k_{x}$ both dipole 
components reinforce each other [Fig. 1(B)]. This near field interference effect is the basis for the selective directional excitation of guided modes by the evanescent components of the dipole field. The directional behaviour that emerges from the evanescent components is a quite counterintuitive result, considering that no preferred direction is observed for the propagating components of the circular dipole, as can be easily shown by noticing that the magnetic field radiated per unit angle, obtained by substituting $k_{x}=k_{0} \sin \theta$ into $H_{y}\left(k_{x}, z\right) d k_{x}$ from Eq. (1), is given by $H_{y}(\theta, z) d \theta=H_{0} e^{\mp i \theta} d \theta$, which has a constant magnitude for all radiating angles $\theta$. From the directional behaviour of the evanescent components, it follows immediately that by placing a structure which supports bound propagating modes with an $\exp \left( \pm i k_{x}^{m} x\right)$ dependence (with $k_{x}^{m}>k_{0}$ ) in the near field of this dipole, the modes propagating towards negative $\mathbf{x}$ will be dominantly excited over the modes propagating in the positive $\mathbf{x}$ direction with a ratio equal to that of the amplitudes of the corresponding evanescent wave components of the dipole $R=\left|H_{y}\left(k_{x}^{m}\right)\right| /\left|H_{y}\left(-k_{x}^{m}\right)\right|$. The opposite directionality is observed if the direction of rotation of the dipole is reversed. The zposition of the dipole does not affect this ratio, since the evanescent decay, $\exp \left(i k_{z} z\right)$ in Eq. (1), affects the fields with both signs of $k_{x}$ equally.

This effect has been demonstrated numerically for two different waveguides using the spectral decomposition of the dipole and the transfer matrix method (23). The first example is a dielectric slab of thickness $s=0.2 \lambda$, with permittivity $\varepsilon_{d}=9+0.1 \mathrm{i}$, placed at a distance $\mathrm{d}=$ $0.1 \lambda$ from the dipole. This slab has a bound waveguided mode that can propagate in opposite directions with the wavevector $\pm k_{x}^{d}= \pm 1.965 k_{0}$ [Fig. 1(B)]. Clearly, the mode with $-k_{x}^{d}$ will be excited by the circular dipole with a much greater amplitude than the one with + $k_{x}^{d^{x}}$ (amplitude ratio $\mathrm{R}=13.4$ ), resulting in unidirectional propagation of the mode within the slab [Fig. 2(B)]. The second example is a semi-infinite metallic medium with permittivity $\varepsilon_{\mathrm{m}}=$ $-2+0.1 \mathrm{i}$, placed at a distance $\mathrm{d}=0.3 \lambda$ from the circular dipole. The air-metal boundary supports counter-propagating SPP modes with wavevectors $\pm k_{x}^{\text {spp }}= \pm 1.41 k_{0}$ [Fig. 1(B)]. Only one of these modes is predominantly excited by the circular dipole (amplitude ratio $\mathrm{R}=$ 5.8), resulting in directional SPP propagation [Fig. 2(C)]. The lobes observed towards the upper right part of the figure correspond to far field radiation from the dipole and its reflection. Animated versions of Figs. 2(A-D) are given in Movies S1-S4.

In both presented examples, a residual non-zero amplitude is still observed for the modes with positive $k_{x}$. This residual wave can be eliminated by using a dipole moment with different relative amplitudes in its $\mathrm{x}$ and $\mathrm{z}$ components (elliptical polarization). Relying on Eq. 1, it is possible to design a dipole with a spatial frequency spectrum such that the field is exactly zero for the mode which needs to be inhibited; e.g., $H_{y}\left(k_{x}^{s p p}\right)=0$ for the wavevector of the mode with positive $k_{x}^{s p p}$. This is the case for $\mathbf{p}_{2 \mathrm{D}}=[1,-0.705 \mathrm{i}]$ showing a zero value at exactly the SPP wavevector $k_{x}^{s p p}=+1.41 k_{0}$ [Fig. 1(B)]. The resulting truly unidirectional excitation of SPP wave is shown in Fig. 1(D), with no coupling to the "undesired" back propagating SPP mode. It must be noted that the finite lifetime of the SPPs due to the Ohmic losses causing broadening in the wavevector spectrum may result in some minor spurious excitation.

To experimentally demonstrate the concept, we show the directional excitation of SPPs on a smooth $50 \mathrm{~nm}$ gold film using a symmetric $130 \mathrm{~nm}$ wide slit illuminated with circularly polarized light. The slit plays the role of the 2D circularly polarized dipole, exciting SPPs on the nearby smooth metal film. 3D scenarios may be realized by considering single defects in 
a film (24) or a nanoparticle placed on a metal surface (25). Because the circular polarization of the dipole is required in a plane perpendicular to the metal surface (Fig. 1(A)), the illumination is chosen at a grazing angle along the slit length [Fig. 3(A)]. The leakage radiation of the SPPs into the glass substrate is recorded as bright spots on a CCD camera placed in a spatially filtered Fourier plane (Fig. S1). In the case of linear polarization, both SPPs are excited equally on both sides of the slit, while for left- and right-handed circular polarization, the SPPs are predominantly excited propagating to the right and left side of the slit, respectively [Fig. 3(C)].

A full set of polarization states were studied rotating a $\lambda / 4$ plate in a linearly polarized excitation beam [Fig. 3(D)]. For linear polarization ( $\lambda / 4$ plate: $0,-90$ and +90 degrees), the expected behaviour of equal excitation of SPPs on both sides of the slit is observed. However, for two circular polarizations of the incident light, SPPs are preferentially excited in one direction. Symmetry requires that the two SPP excitation curves are mirror images of each other, which is clearly observed in the experiment. Small deviations from theoretical predictions obtained for an ideal dipole can be expected to originate from the finite size and shape of the slit, whose dipolar behaviour is not equivalent for fields polarized across the slit and perpendicularly to it. This prevents achieving full directionality and contributes to the observed background intensity [Fig. 3(D)]. A complete control of the polarization ellipse of incoming light $(10,11)$ would optimize the device performance, which following symmetry considerations (22) could potentially achieve a 1:0 contrast ratio. Similarly, directional SPP excitation can be achieved with a slit grating using the same approach (Fig. S2).
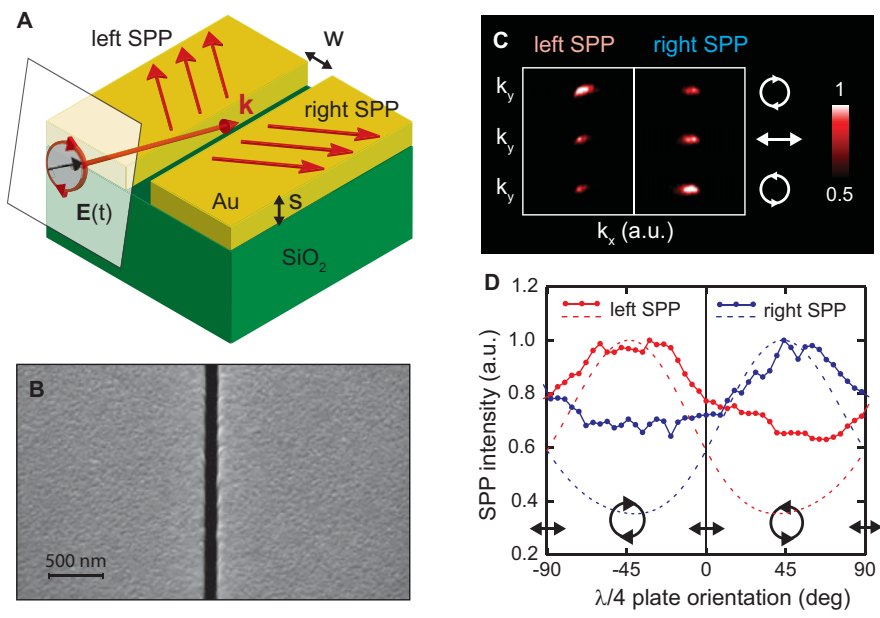

Figure 3. (A) Schematics of the experiment: a plane wave is incident at nearly grazing angle (70 degrees) onto a slit in a metal film. (B) Scanning electron micrograph of the slit. (C) Spatially filtered Fourier plane images of the SPP leakage radiation collected from the sample for different polarization states of the illuminating light. The bright spots correspond to the points on the SPP wavevector arc for the Au/air interface with $\mathrm{k}_{\mathrm{y}}$ matching the incident wavevector. (D) Experimental (solid lines) and simulated (dashed lines) dependences of the intensity of left and right excited SPPs on the polarization of the illuminating light. 
We have described a fundamental example of vectorial near field interference using a simple circularly or elliptically polarized dipole to achieve directional broadband excitation of guided modes. The underlying physics holds potential and wide applicability to other optical processes. Useful applications may be in the excitation of photonic guided modes exhibiting directional dependence with the direction of the input circularly polarized light, enabling compact nanoscale light sources that could efficiently absorb incoming illumination and couple it to the modes of Si-photonic or plasmonic guides in a desired single direction. It also opens exciting possibilities for directional switching, polarization sorting, and processing of polarization-encoded information, including polarization entangled optical qubits.

Acknowledgements: This work has been supported in part by EPSRC (grant EP/H000917/2). F. J. Rodríguez-Fortuño acknowledges support from grant FPI of GV. A. Martínez acknowledges financial support from Spanish government (contracts Consolider EMET CSD2008-00066 and TEC2011-28664-C02-02). P. Ginzburg acknowledges the Royal Society for a Newton International Fellowship.

\section{References and Notes}

1. N. Yu et al., Science 334, 333-337 (2011).

2. X. Ni, N. K. Emani, A. V Kildishev, A. Boltasseva, V. M. Shalaev, Science 335, 427 (2012).

3. L. Novotny, B. Hecht, Principles of Nano-Optics (Cambridge University Press, 2011).

4. R. Merlin, Science 317, 927-929 (2007).

5. J. B. Pendry, Phys. Rev. Lett. 85, 3966-3969 (2000).

6. L. E. Helseth, Opt. Commun. 281, 1981-1985 (2008).

7. G. V. Eleftheriades, A. M. H. Wong, IEEE Microw. Wirel. Co. 18, 236-238 (2008).

8. J. Y. Lee et al., Nature 460, 498-501 (2009).

9. M. Stockman, S. Faleev, D. Bergman, Phys. Rev. Lett. 88, 067402 (2002).

10. M. Aeschlimann et al., New Journal of Physics 14, 033030 (2012).

11. M. Sukharev, T. Seideman, Nano Lett. 6, 715-719 (2006).

12. W. L. Barnes, A. Dereux, T. W. Ebbesen, Nature 424, 824-830 (2003).

13. J. a Schuller et al., Nat. Mater. 9, 193-204 (2010).

14. H. Kim, B. Lee, Plasmonics 4, 153-159 (2009).

15. N. Bonod, E. Popov, L. Li, B. Chernov, Opt. Express 15, 11427-11432 (2007).

16. J.-S. Bouillard, S. Vilain, W. Dickson, G. A. Wurtz, A. V Zayats, Scientific reports 2, 829 (2012).

17. X. Li, Q. Tan, B. Bai, G. Jin, Appl. Phys. Lett. 98, 251109 (2011).

18. I. P. Radko et al., Opt. Express 17, 7228-7232 (2009).

19. Y. Liu et al., Nano Lett. 12, 4853-4858 (2012).

20. A. G. Curto et al., Science 329, 930-933 (2010). 
21. G. Roelkens et al., J. Nanosci. Nanotechnol. 10, 1551-1562 (2010).

22. See Supplemental Material at http://www.sciencemag.org/content/340/6130/328/suppl/DC1 for Movies S1-S4, materials and methods, and supplementary text.

23. W. N. Hansen, J. Opt. Soc. Am. 58, 380-388 (1968).

24. N. Rotenberg et al., Phys. Rev. Lett. 108, 127402 (2012).

25. Z. Ruan, S. Fan, Phys. Rev. Lett. 105, 013901 (2010). 


\section{General discussion of results and conclusions}

As THE READER WILL HAVE NOTICED, the research work presented for this thesis deals with plasmonic phenomena of very diverse nature. One could think that a thesis focused into a very specific application or device would give the best results. However, given the whirlwind of activity and new findings surrounding plasmonics and metamaterials, we believe that such a thesis would miss the opportunity of finding novel ideas and structures that potentially remain hidden in the various corners of this broad field which are yet to be explored. Therefore, the "guiding compass" of this thesis was our scientific curiosity and the intuition of potentially new results, not limiting ourselves to a single application or topic.

Even if the results of this thesis cover a wide variety of applications, from polarization conversion to sensing, their common background is always plasmonics, meaning that the acquired knowledge is shared: it is clear that the effort thrown into each of our researches supports and inspires the work in all others.

- We proposed two original contributions to plasmonic negative index metamaterials based on U-shaped nanowires and coaxial plasmonic waveguides. Although the U-shaped nanowires seem to be less practical, as learned after our experimental realization, the coaxial plasmonic waveguide was given consideration in other works as a viable candidate, published in Nature Materials, for a wide angle broadband negative index metamaterial working in the visible spectrum [147]. The design of both metamaterials was possible through the knowledge of the dispersion relations and the field and current distributions of complex plasmonic waveguides, together with the effects of a finite size.

- We describe the use of split ring resonators inside a photonic crystal for light stopping and time reversal of pulses. This idea was possible through the study of the dispersion relation of periodic structures, very much related to that of finite structures mentioned above.

- We explore a method of low-loss plasmonic propagation by making use of multimode interference achieving the reduction of the currents in the metallic 
walls. The method shows an extremely high reduction of the losses, at the cost however of a lower confinement. The idea could prove useful in the design of structures in which the metallic walls are unavoidable, such as electrodes in an active photonic circuit. This study required again the knowledge of the field profiles and dispersion relations in plasmonic waveguides.

- We theoretically proposed and experimentally tested a localized resonance plasmonic sensor based on a simple nanocross geometry which exhibits a reasonably large sensitivity. This was possible thanks to the understanding of LSPRs gained through our first studies of metamaterials.

- The same nanocross structure used for sensing was useful in our design of the plasmonic analog of the Hanle effect for the creation of an extremely compact and potentially tunable polarization conversion metamaterial, which resulted on a publication in the journal Nano Letters ${ }^{5}$.

- We showed a metamaterial analogue of the quantum Meissner levitation working thanks to the field expulsion in $\varepsilon$-near-zero metamaterials. The Green function and the plane wave decomposition of a dipole where the foundations for this work $^{6}$.

- We demonstrated theoretically and experimentally the unidirectional near field excitation of photonic modes by a circularly polarized dipole. This study was supported by the knowledge of plane wave decomposition of a dipole over a substrate mentioned above. Our result is published in the prestigious journal Science $^{7}$, and it gives a new fundamental insight into near field interference, by providing an example which can be initially regarded as a non-intuitive result. The work could give rise to many exciting devices that could achieve polarization splitting, generation and analysis of polarized light, or processing of polarization encoded information, among others.

\section{Future work}

Many of the research topics in the thesis allow for future work and further results.

- The experimental demonstration of a negative index metamaterial based on coaxial plasmonic waveguides is already being pursued by other groups. The experimental demonstration of the plasmonic analogue to the Hanle effect combined with an external tuning of induced losses could form a potentially efficient plasmonic switching device.

\footnotetext{
5Nano Letters' impact factor was 13.198 in 2011.

${ }^{6}$ Spatial-frequency Fourier analysis was initially used for many of the works on metallic hole arrays in which the student collaborated as co-author, as well as for the performance analysis of lossy lenses.

${ }^{7}$ Science impact factor was 31.201 in 2011.
} 
- Regarding the metamaterial analogue of Meissner levitation, not only an experimental demonstration would constitute an important result, but we also envision new extensions of the concept. For example, the imitation of flux-pinning effects -when lines of magnetic field penetrate certain types of superconductors and give rise to very stable levitation [279], sometimes described as if the levitating magnet was inserted inside a pile of sand-could be mimicked with air channels in ENZ media, and we would like to investigate such curious effect.

- Finally, the unidirectional excitation of guided modes by a circularly polarized dipole is inspiring us in the creation of many new devices: we are currently working on several ideas such as a demonstration at microwave frequencies in a two-dimensional circuit, and a useful polarization splitter device integrated in a nanophotonic silicon waveguide.

\section{Concluding remarks}

The results listed above constitute our original contributions to the fields of plasmonics and metamaterials that resulted in publications on international scientific journals. We would like to think that those achievements are an indication that our initial goal of contributing to this field of knowledge has been met. However, we would also like to mention that, apart from those results, a substantial amount of theoretical and experimental work was directed to ideas which sometimes did not end up having positive outcomes, such as the attempts at an infrared magnetoinductive lens, the design of active plasmonic switches, the measurement of localized plasmonic resonances in top of a silicon waveguide, and the study of Förster Resonance Energy Transfer (FRET) enhancement on anisotropic rod metamaterials, among others. This is important to note because almost all successful ideas are preceded by tentative exploratory ones.

As a final remark, during these years of research, this doctoral student realized that the possibility of easily tuning the many variables of a given problem at hand and obtaining an immediate feedback on its effects, allows one to experiment and play comfortably with the designs. In plasmonics, the immediate feedback can be achieved by fast analytic or numeric algorithms with intuitive user interfaces ${ }^{8}$, leading to new understanding and new ideas that would have otherwise remained hidden ${ }^{9}$. In short, creativity thrives under a "playful" mood ${ }^{10}$.

\footnotetext{
${ }^{8}$ This was done wherever possible throughout this thesis: plasmonic dispersion relations, transfer matrix method, and automated electromagnetic simulator scripts were written for the easy tuning and the fast analysis of structures.

${ }^{9}$ The importance of an immediate feedback in the creative process is fundamentally defended by Bret Victor. See his speech: "Inventing on Principle" available online.

${ }^{10}$ The importance of a playful mood is very well described by John Cleese in his speech: "On creativity" available online.
} 


\section{References}

[1] D. Pile, “Milestone 20: (1998) Plasmonics," Nature (2010), 10.1038/nmat2657.

[2] Editorial, "Surface Plasmon Resurrection," Nature Photonics 6, 707 (2012).

[3] E. Ozbay, "Plasmonics: merging photonics and electronics at nanoscale dimensions." Science 311, 189 (2006).

[4] J. N. Anker, W. P. Hall, O. Lyandres, N. C. Shah, J. Zhao, and R. P. Van Duyne, "Biosensing with plasmonic nanosensors." Nature materials 7, 442 (2008).

[5] S. Kawata, Y. Inouye, and P. Verma, "Plasmonics for near-field nano-imaging and superlensing," Nature Photonics 3, 388 (2009).

[6] W. L. Barnes, A. Dereux, and T. W. Ebbesen, "Surface plasmon subwavelength optics." Nature 424, 824 (2003).

[7] N. I. Zheludev, "Applied physics. The road ahead for metamaterials." Science 328, 582 (2010).

[8] J. B. Pendry, "Negative Refraction Makes a Perfect Lens," Physical Review Letters 85, 3966 (2000).

[9] D. Schurig, J. J. Mock, B. J. Justice, S. A. Cummer, J. B. Pendry, A. F. Starr, and D. R. Smith, "Metamaterial electromagnetic cloak at microwave frequencies." Science 314, 977 (2006).

[10] W. Cai, U. K. Chettiar, A. V. Kildishev, and V. M. Shalaev, “Optical cloaking with metamaterials," Nature Photonics 1, 224 (2007).

[11] J. Heber, “Milestone 21 (2000) Metamaterials," Nature (2010), 10.1038/nmat2658.

[12] C. M. Soukoulis and M. Wegener, "Past achievements and future challenges in the development of three-dimensional photonic metamaterials," Nature Photonics 5, 523 (2011). 
[13] S. A. Maier, Plasmonics: Fundamentals and Applications (Springer, 2010).

[14] L. Novotny and B. Hecht, Principles of Nano-Optics (Cambridge University Press, 2011).

[15] G. Mie, "Beiträge zur Optik trüber Medien, speziell kolloidaler Metallösungen," Annalen der Physik 330, 377 (1908).

[16] A. Sommerfeld, "Ueber die Fortpflanzung elektrodynamischer Wellen längs eines Drahtes," Annalen der Physik und Chemie 303, 233 (1899).

[17] A. Sommerfeld, Partial Differential Equations in Physics (Academic Press, 1953).

[18] R. Wood, "On a remarkable case of uneven distribution of light in a diffraction grating spectrum," Philosophical Magazine Series 6 4, 396 (1902).

[19] U. Fano, "The Theory of Anomalous Diffraction Gratings and of Quasi-Stationary Waves on Metallic Surfaces (Sommerfeld's Waves)," Journal of the Optical Society of America 31, 213 (1941).

[20] R. Ritchie, "Plasma Losses by Fast Electrons in Thin Films," Physical Review 106, 874 (1957).

[21] R. Ritchie, E. Arakawa, J. Cowan, and R. Hamm, "Surface-Plasmon Resonance Effect in Grating Diffraction," Physical Review Letters 21, 1530 (1968).

[22] E. Kretschmann and H. Raether, "Radiative decay of non-radiative surface plasmons excited by light," Zeitschrift für Naturforschung Section A A Journal Of Physical Sciences 23, 2135 (1968).

[23] J. A. Schuller, E. S. Barnard, W. Cai, Y. C. Jun, J. S. White, and M. L. Brongersma, "Plasmonics for extreme light concentration and manipulation." Nature materials 9, 193 (2010).

[24] A. J. Haes and R. P. Van Duyne, "A unified view of propagating and localized surface plasmon resonance biosensors." Analytical and bioanalytical chemistry 379,920 (2004).

[25] M. E. Stewart, C. R. Anderton, L. B. Thompson, J. Maria, S. K. Gray, J. a. Rogers, and R. G. Nuzzo, "Nanostructured plasmonic sensors." Chemical reviews 108, 494 (2008).

[26] K. A. Willets and R. P. Van Duyne, "Localized surface plasmon resonance spectroscopy and sensing." Annual review of physical chemistry 58, 267 (2007).

[27] G. Wurtz and A. Zayats, "Nonlinear surface plasmon polaritonic crystals," Laser \& Photonics Review 2, 125 (2008). 
[28] G. T. Reed and A. P. Knights, Silicon Photonics: An Introduction (Wiley, 2004).

[29] N. Fang, H. Lee, C. Sun, and X. Zhang, "Sub-diffraction-limited optical imaging with a silver superlens." Science 308, 534 (2005).

[30] W. Srituravanich, L. Pan, Y. Wang, C. Sun, D. B. Bogy, and X. Zhang, "Flying plasmonic lens in the near field for high-speed nanolithography." Nature nanotechnology 3, 733 (2008).

[31] J.-J. Greffet, “Nanoantennas for light emission.” Science 308, 1561 (2005).

[32] L. Novotny and N. van Hulst, "Antennas for light," Nature Photonics 5, 83 (2011).

[33] C. Genet and T. W. Ebbesen, "Light in tiny holes." Nature 445, 39 (2007).

[34] V. G. Veselago and E. E. Narimanov, "The left hand of brightness: past, present and future of negative index materials." Nature materials 5, 759 (2006).

[35] A. Boltasseva and H. A. Atwater, "Materials science. Low-loss plasmonic metamaterials." Science 331, 290 (2011).

[36] J. D. Jackson, Classical Electrodynamics (Wiley, 1998).

[37] F. J. García de Abajo, "Nonlocal Effects in the Plasmons of Strongly Interacting Nanoparticles, Dimers, and Waveguides," Journal of Physical Chemistry C 112, 17983 (2008).

[38] C. Ciracì, R. T. Hill, J. J. Mock, Y. Urzhumov, A. I. Fernández-Domínguez, S. A. Maier, J. B. Pendry, A. Chilkoti, and D. R. Smith, "Probing the ultimate limits of plasmonic enhancement." Science 337, 1072 (2012).

[39] A. D. Rakic, A. B. Djurisic, J. M. Elazar, and M. L. Majewski, "Optical properties of metallic films for vertical-cavity optoelectronic devices." Applied optics 37, 5271 (1998).

[40] E. D. Palik, Handbook of Optical Constants of Solids (Academic Press, San Diego, 1998).

[41] P. B. Johnson and R. W. Christy, "Optical Constants of the Noble Metals," Physical Review B 6, 4370 (1972).

[42] C. A. Balanis, Advanced Engineering Electromagnetics (Wiley, 1989).

[43] E. Economou, "Surface Plasmons in Thin Films," Physical Review 182, 539 (1969).

[44] C. Pfeiffer, E. Economou, and K. Ngai, "Surface polaritons in a circularly cylindrical interface: Surface plasmons," Physical Review B 10, 3038 (1974). 
[45] A. Otto, "Excitation of nonradiative surface plasma waves in silver by the method of frustrated total reflection," Zeitschrift für Physik 216, 398 (1968).

[46] B. Hecht, H. Bielefeldt, L. Novotny, Y. Inouye, and D. Pohl, "Local Excitation, Scattering, and Interference of Surface Plasmons," Physical Review Letters 77, 1889 (1996).

[47] A. Sihvola, "Metamaterials in electromagnetics,” Metamaterials 1, 2 (2007).

[48] L. Solymar and E. Shamonina, Waves in Metamaterials (Oxford University Press, USA, 2009).

[49] W. L. Bragg, "The diffraction of short electromagnetic waves by a crystal," Proceedings of the Cambridge Philosophical Society 17, 43 (1913).

[50] D. J. Griffiths, Introduction to Electrodynamics (Prentice Hall, New Jersey, 1999).

[51] A. Ishimaru, Electromagnetic Wave Propagation, Radiation, and Scattering (Prentice Hall, 1990).

[52] L. Brillouin, Wave propagation in periodic structures (Dover Publications, New York, 1953).

[53] O. F. Mossotti, “Discussione analitica: sull'influenza che l'azione di un mezzo dielettrico ha sulla distribuzione dell'elettricita alla superficie di piu corpi elettrici disseminati in ess," Memorie della Societa Italiana delle Scienze XXIV(2), 49 (1850).

[54] R. Clausius and B. Dibner, Die mechanische behandlung der electricität. Vol. 2 (Friedrich Vieweg und Sohn, 1879).

[55] K. Busch, G. von Freymann, S. Linden, S. Mingaleev, L. Tkeshelashvili, and M. Wegener, "Periodic nanostructures for photonics," Physics Reports 444, 101 (2007).

[56] S. John, "Strong localization of photons in certain disordered dielectric superlattices," Physical Review Letters 58, 2486 (1987).

[57] E. Yablonovitch, "Inhibited Spontaneous Emission in Solid-State Physics and Electronics," Physical Review Letters 58, 2059 (1987).

[58] C. E. Kriegler, M. S. Rill, S. Linden, and M. Wegener, "Bianisotropic Photonic Metamaterials," IEEE Journal of Selected Topics in Quantum Electronics 16, 367 (2010).

[59] L. Lewin, "The Electrical Constants of a Material Loaded with Spherical Particles," Journal of the Institution of Electrical Engineers - Part III: Radio and Communication Engineering 94, 65 (1947). 
[60] G. H. B. Thompson, "Unusual Waveguide Characteristics associated with the Apparent Negative Permeability obtainable in Ferrites," Nature 175, 1135 (1955).

[61] W. Rotman, "Plasma simulation by artificial dielectrics and parallel-plate media," IRE Transactions on Antennas and Propagation 10, 82 (1962).

[62] J. Brown, "The design of metallic delay dielectrics," Proceedings of the IEEE-Part III: Radio and Communication Engineering 97, 45 (1950).

[63] J. Pendry, A. Holden, W. Stewart, and I. Youngs, "Extremely Low Frequency Plasmons in Metallic Mesostructures," Physical Review Letters 76, 4773 (1996).

[64] J. B. Pendry, A. J. Holden, D. Robbins, and W. J. Stewart, “Magnetism from conductors and enhanced nonlinear phenomena," IEEE Transactions on Microwave Theory and Techniques 47, 2075 (1999).

[65] E. Shamonina and L. Solymar, "Metamaterials: How the subject started," Metamaterials 1, 12 (2007).

[66] V. G. Veselago, "The electrodynamics of substances with simultaneously negative values of $\epsilon$ and $\mu$," Soviet Physics Uspekhi 10, 509 (1968).

[67] K. L. Tsakmakidis, A. D. Boardman, and O. Hess, "'Trapped rainbow' storage of light in metamaterials." Nature 450, 397 (2007).

[68] D. Schurig and D. Smith, "Negative index lens aberrations," Physical Review E 70, 065601 (2004).

[69] U. Leonhardt and T. G. Philbin, "Quantum levitation by left-handed metamaterials," New Journal of Physics 9, 254 (2007).

[70] W. N. Hansen, "Electric Fields Produced by the Propagation of Plane Coherent Electromagnetic Radiation in a Stratified Medium," Journal of the Optical Society of America 58, 380 (1968).

[71] The News and Editorial Staffs, “The Runners-Up," Science 302, 2039 (2003).

[72] Z. Ye, "Optical transmission and reflection of perfect lenses by left handed materials," Physical Review B 67, 193106 (2003).

[73] N. Garcia and M. Nieto-Vesperinas, "Left-Handed Materials Do Not Make a Perfect Lens," Physical Review Letters 88, 207403 (2002).

[74] V. A. Podolskiy and E. E. Narimanov, "Near-sighted superlens," Optics Letters 30, 75 (2005).

[75] J. Marqués, R.Baena, "Effect of losses and dispersion on the focusing properties of left-handed media," Microwave and Optical Technology Letters 41, 290 (2004). 
[76] O. E. French, K. I. Hopcraft, and E. Jakeman, "Perturbation on the perfect lens: the near-perfect lens," New Journal of Physics 8, 271 (2006).

[77] D. R. Smith, D. Schurig, M. Rosenbluth, S. Schultz, S. A. Ramakrishna, and J. B. Pendry, "Limitations on subdiffraction imaging with a negative refractive index slab," Applied Physics Letters 82, 1506 (2003).

[78] A. Lagarkov and V. Kissel, "Near-Perfect Imaging in a Focusing System Based on a Left-Handed-Material Plate," Physical Review Letters 92, 077401 (2004).

[79] K. Aydin and E. Ozbay, "Left-handed metamaterial based superlens for subwavelength imaging of electromagnetic waves," Applied Physics A 87, 137 (2007).

[80] A. Grbic and G. Eleftheriades, "Overcoming the Diffraction Limit with a Planar Left-Handed Transmission-Line Lens," Physical Review Letters 92, 117403 (2004).

[81] M. J. Freire and R. Marqués, "Planar magnetoinductive lens for threedimensional subwavelength imaging," Applied Physics Letters 86, 182505 (2005).

[82] M. J. Freire and R. Marqués, "Optimizing the magnetoinductive lens: Improvement, limits, and possible applications," Journal of Applied Physics 103, 013115 (2008).

[83] M. J. Freire, L. Jelinek, R. Marqués, and M. Lapine, "On the applications of $\mu=-1$ metamaterial lenses for magnetic resonance imaging." Journal of magnetic resonance 203, 81 (2010).

[84] A. Salandrino and N. Engheta, "Far-field subdiffraction optical microscopy using metamaterial crystals: Theory and simulations," Physical Review B 74, 075103 (2006).

[85] Z. Jacob, L. V. Alekseyev, and E. Narimanov, “Optical Hyperlens: Far-field imaging beyond the diffraction limit," Optics Express 14, 8247 (2006).

[86] J. B. Pendry and S. A. Ramakrishna, "Near-field lenses in two dimensions," Journal of Physics: Condensed Matter 14, 8463 (2002).

[87] Z. Liu, H. Lee, Y. Xiong, C. Sun, and X. Zhang, "Far-field optical hyperlens magnifying sub-diffraction-limited objects." Science 315, 1686 (2007).

[88] R. A. Depine and A. Lakhtakia, "A new condition to identify isotropic dielectricmagnetic materials displaying negative phase velocity," Microwave and Optical Technology Letters 41, 315 (2004). 
[89] D. Smith, W. Padilla, D. Vier, S. Nemat-Nasser, and S. Schultz, "Composite Medium with Simultaneously Negative Permeability and Permittivity," Physical Review Letters 84, 4184 (2000).

[90] K. Aydin, I. Bulu, K. Guven, M. Kafesaki, C. M. Soukoulis, and E. Ozbay, “Investigation of magnetic resonances for different split-ring resonator parameters and designs," New Journal of Physics 7, 168 (2005).

[91] J. Baena, J. Bonache, F. Martin, R. Sillero, F. Falcone, T. Lopetegi, M. Laso, J. Garcia-Garcia, I. Gil, M. Portillo, and M. Sorolla, "Equivalent-circuit models for split-ring resonators and complementary split-ring resonators coupled to planar transmission lines," IEEE Transactions on Microwave Theory and Techniques 53, 1451 (2005).

[92] T. F. Gundogdu, I. Tsiapa, A. Kostopoulos, G. Konstantinidis, N. Katsarakis, R. S. Penciu, M. Kafesaki, E. N. Economou, T. Koschny, and C. M. Soukoulis, "Experimental demonstration of negative magnetic permeability in the farinfrared frequency regime," Applied Physics Letters 89, 084103 (2006).

[93] L. Roglá, J. Carbonell, and V. Boria, "Study of equivalent circuits for open-ring and split-ring resonators in coplanar waveguide technology," IET Microwaves, Antennas \& Propagation 1, 170 (2007).

[94] K. Aydin and E. Özbay, "Experimental and numerical analyses of the resonances of split ring resonators," physica status solidi (b) 244, 1197 (2007).

[95] J. D. Baena, L. Jelinek, and R. Marqués, "Reducing losses and dispersion effects in multilayer metamaterial tunnelling devices," New Journal of Physics 7, 166 (2005).

[96] R. Marqués, J. Martel, F. Mesa, and F. Medina, "A new 2D isotropic lefthanded metamaterial design: Theory and experiment," Microwave and Optical Technology Letters 35, 405 (2002).

[97] P. Gay-Balmaz and O. J. F. Martin, "Efficient isotropic magnetic resonators," Applied Physics Letters 81, 939 (2002).

[98] S. O'Brien and J. B. Pendry, "Magnetic activity at infrared frequencies in structured metallic photonic crystals," Journal of Physics: Condensed Matter 14, 6383 (2002).

[99] A. Andryieuski, R. Malureanu, and A. Lavrinenko, "Wave propagation retrieval method for metamaterials: Unambiguous restoration of effective parameters," Physical Review B 80, 193101 (2009).

[100] S. Ha, A. a. Sukhorukov, K. B. Dossou, L. C. Botten, C. M. de Sterke, and Y. S. Kivshar, "Bloch-mode extraction from near-field data in periodic waveguides." Optics letters 34, 3776 (2009). 
[101] M. G. Silveirinha, “Time domain homogenization of metamaterials," Physical Review B 83, 165104 (2011).

[102] A. Andryieuski, S. Ha, A. Sukhorukov, Y. Kivshar, and A. Lavrinenko, “Blochmode analysis for retrieving effective parameters of metamaterials," Physical Review B 86, 035127 (2012).

[103] D. R. Smith and S. Schultz, "Determination of effective permittivity and permeability of metamaterials from reflection and transmission coefficients," Physical Review B 65, 195104 (2002).

[104] X. Chen, T. Grzegorczyk, B.-i. Wu, J. Pacheco, and J. Kong, "Robust method to retrieve the constitutive effective parameters of metamaterials," Physical Review E 70, 016608 (2004).

[105] D. R. Smith, D. C. Vier, T. Koschny, and C. M. Soukoulis, “Electromagnetic parameter retrieval from inhomogeneous metamaterials," Physical Review E 71, 036617 (2005).

[106] A. M. Nicolson and G. F. Ross, "Measurement of the Intrinsic Properties of Materials by Time-Domain Techniques," IEEE Transactions on Instrumentation and Measurement 19, 377 (1970).

[107] W. Weir, "Automatic measurement of complex dielectric constant and permeability at microwave frequencies," Proceedings of the IEEE 62, 33 (1974).

[108] T. Koschny, P. Markoš, D. Smith, and C. Soukoulis, "Resonant and antiresonant frequency dependence of the effective parameters of metamaterials," Physical Review E 68, 065602 (2003).

[109] J. Woodley and M. Mojahedi, "On the signs of the imaginary parts of the effective permittivity and permeability in metamaterials," Journal of the Optical Society of America B 27, 1016 (2010).

[110] C. R. Simovski, “On electromagnetic characterization and homogenization of nanostructured metamaterials," Journal of Optics 13, 013001 (2011).

[111] C. Menzel, C. Rockstuhl, T. Paul, F. Lederer, and T. Pertsch, "Retrieving effective parameters for metamaterials at oblique incidence," Physical Review B 77, 195328 (2008).

[112] S. Linden, C. Enkrich, M. Wegener, J. Zhou, T. Koschny, and C. M. Soukoulis, "Magnetic response of metamaterials at 100 terahertz." Science 306, 1351 (2004).

[113] S. Zhang, W. Fan, B. Minhas, A. Frauenglass, K. Malloy, and S. Brueck, "Midinfrared Resonant Magnetic Nanostructures Exhibiting a Negative Permeability," Physical Review Letters 94, 037402 (2005). 
[114] C. Enkrich, F. Pérez-Willard, D. Gerthsen, J. F. Zhou, T. Koschny, C. M. Soukoulis, M. Wegener, and S. Linden, "Focused-Ion-Beam Nanofabrication of NearInfrared Magnetic Metamaterials," Advanced Materials 17, 2547 (2005).

[115] M. W. Klein, C. Enkrich, M. Wegener, C. M. Soukoulis, and S. Linden, "Single-slit split-ring resonators at optical frequencies: limits of size scaling," Optics Letters 31,1259 (2006).

[116] J. Zhou, T. Koschny, M. Kafesaki, E. N. Economou, J. B. Pendry, and C. M. Soukoulis, "Saturation of the Magnetic Response of Split-Ring Resonators at Optical Frequencies," Physical Review Letters 95, 223902 (2005).

[117] V. Delgado, O. Sydoruk, E. Tatartschuk, R. Marqués, M. Freire, and L. Jelinek, "Analytical circuit model for split ring resonators in the far infrared and optical frequency range," Metamaterials 3, 57 (2009).

[118] C. Rockstuhl, F. Lederer, C. Etrich, T. Zentgraf, J. Kuhl, and H. Giessen, “On the reinterpretation of resonances in split-ring-resonators at normal incidence," Optics Express 14, 8827 (2006).

[119] V. M. Shalaev, W. Cai, U. K. Chettiar, H.-K. Yuan, A. K. Sarychev, V. P. Drachev, and A. V. Kildishev, "Negative index of refraction in optical metamaterials," Optics Letters 30, 3356 (2005).

[120] M. Beruete, M. Sorolla, and I. Campillo, "Left-handed extraordinary optical transmission through a photonic crystal of subwavelength hole arrays," Optics Express 14, 5445 (2006).

[121] M. Navarro-Cia, M. Beruete, M. Sorolla, and I. Campillo, "Negative refraction in a prism made of stacked subwavelength hole arrays," Optics Express 16, 560 (2008).

[122] M. Beruete, M. Navarro-Cía, and M. Sorolla, "High numerical aperture and low-loss negative refraction based on the fishnet rich anisotropy," Photonics and Nanostructures - Fundamentals and Applications 10, 263 (2012).

[123] M. Navarro-Cía, C. García-Meca, M. Beruete, A. Martínez, and M. Sorolla, "Dual-band double-negative-index fishnet metamaterial at millimeter-waves," Optics Letters 36, 4245 (2011).

[124] G. Dolling, C. Enkrich, M. Wegener, C. M. Soukoulis, and S. Linden, "Lowloss negative-index metamaterial at telecommunication wavelengths," Optics Letters 31, 1800 (2006).

[125] C. García-Meca, J. Hurtado, J. Martí, A. Martínez, W. Dickson, and A. V. Zayats, "Low-Loss Multilayered Metamaterial Exhibiting a Negative Index of Refraction at Visible Wavelengths," Physical Review Letters 106, 067402 (2011). 
[126] T. Xu, M. Abashin, A. Agrawal, K. Chau, and H. Lezec, “Three-dimensional Negative Index of Refraction in the Ultraviolet," in Frontiers in Optics Conference (Rochester, 2012).

[127] A. Alù, A. Salandrino, and N. Engheta, "Negative effective permeability and left-handed materials at optical frequencies," Optics Express 14, 1557 (2006).

[128] C. R. Simovski and S. A. Tretyakov, "Towards isotropic negative magnetics in the visible range," ArXiv , 17 (2008), arXiv:0806.3512 .

[129] C. García-Meca, R. Ortuño, R. Salvador, A. Martínez, and J. Martí, “Lowloss single-layer metamaterial with negative index of refraction at visible wavelengths." Optics express 15, 9320 (2007).

[130] S. Martinos and E. Economou, "Excitation of surface plasmons in cylinders by electrons," Physical Review B 24, 6908 (1981).

[131] J. Takahara, S. Yamagishi, H. Taki, A. Morimoto, and T. Kobayashi, “Guiding of a one-dimensional optical beam with nanometer diameter," Optics Letters 22, 475 (1997).

[132] G. Schider, J. Krenn, A. Hohenau, H. Ditlbacher, A. Leitner, F. Aussenegg, W. Schaich, I. Puscasu, B. Monacelli, and G. Boreman, "Plasmon dispersion relation of Au and Ag nanowires," Physical Review B 68, 155427 (2003).

[133] R. M. Dickson and L. A. Lyon, "Unidirectional Plasmon Propagation in Metallic Nanowires," The Journal of Physical Chemistry B 104, 6095 (2000).

[134] F. Neubrech, T. Kolb, R. Lovrincic, G. Fahsold, A. Pucci, J. Aizpurua, T. W. Cornelius, M. E. Toimil-Molares, R. Neumann, and S. Karim, "Resonances of individual metal nanowires in the infrared," Applied Physics Letters 89, 253104 (2006).

[135] H. Ditlbacher, A. Hohenau, D. Wagner, U. Kreibig, M. Rogers, F. Hofer, F. R. Aussenegg, and J. R. Krenn, "Silver nanowires as surface plasmon resonators," Phys. Rev. Lett. 95, 257403 (2005).

[136] S. Hasan, R. Filter, A. Ahmed, R. Vogelgesang, R. Gordon, C. Rockstuhl, and F. Lederer, "Relating localized nanoparticle resonances to an associated antenna problem," Physical Review B 84, 195405 (2011).

[137] C. Rockstuhl, T. Zentgraf, E. Pshenay-severin, J. Petschulat, A. Chipouline, J. Kuhl, H. Giessen, T. Pertsch, and F. Lederer, "The origin of magnetic polarizability in metamaterials at optical frequencies - an electrodynamic approach," Optics Express 15, 8871 (2007). 
[138] M. M. I. Saadun and N. Engheta, “Theoretical Study of Electromagnetic Properties of Non-Local $\Omega$ Media," Progress In Electromagnetics Research 9, 351 (1994).

[139] M. M. I. Saadoun and N. Engheta, "A reciprocal phase shifter using novel pseudochiral or $\omega$ medium," Microwave and Optical Technology Letters 5, 184 (1992).

[140] P. Tournois and V. Laude, "Negative group velocities in metal-film optical waveguides," Optics Communications 137, 41 (1997).

[141] A. Alù and N. Engheta, "Optical nanotransmission lines: synthesis of planar left-handed metamaterials in the infrared and visible regimes," Journal of the Optical Society of America B 23, 571 (2006).

[142] H. Shin and S. Fan, "All-Angle Negative Refraction for Surface Plasmon Waves Using a Metal-Dielectric-Metal Structure," Physical Review Letters 96, 073907 (2006).

[143] H. Shin, M. F. Yanik, S. Fan, R. Zia, and M. L. Brongersma, “Omnidirectional resonance in a metal-dielectric-metal geometry," Applied Physics Letters 84, 4421 (2004).

[144] H. J. Lezec, J. A. Dionne, and H. A. Atwater, "Negative refraction at visible frequencies." Science 316, 430 (2007).

[145] J. A. Dionne, E. Verhagen, A. Polman, and H. A. Atwater, "Are negative index materials achievable with surface plasmon waveguides? A case study of three plasmonic geometries," Optics Express 16, 19001 (2008).

[146] P. B. Catrysse and S. Fan, "Understanding the dispersion of coaxial plasmonic structures through a connection with the planar metal-insulator-metal geometry," Applied Physics Letters 94, 231111 (2009).

[147] S. P. Burgos, R. de Waele, A. Polman, and H. A. Atwater, "A single-layer wideangle negative-index metamaterial at visible frequencies." Nature materials $\mathbf{9}$, 407 (2010).

[148] R. de Waele, S. P. Burgos, H. A. Atwater, and A. Polman, "Negative refractive index in coaxial plasmon waveguides," Optics Express 18, 12770 (2010).

[149] E. Verhagen, R. de Waele, L. Kuipers, and A. Polman, “Three-Dimensional Negative Index of Refraction at Optical Frequencies by Coupling Plasmonic Waveguides," Physical Review Letters 105, 223901 (2010).

[150] R. P. Feynman, R. B. Leighton, and M. Sands, The Feynman Lectures on Physics, Vol. 2 (Addison-Wesley, 1964). 
[151] H. Beutler, "Über Absorptionsserien von Argon, Krypton und Xenon zu Termen zwischen den beiden Ionisierungsgrenzen ^2 P_3^\{2/0\} und `2 P_1^\{2/0\}," Zeitschrift für Physik 93, 177 (1935).

[152] U. Fano, "Sullo spettro di assorbimento dei gas nobili presso il limite dello spettro d'arco," Il Nuovo Cimento 12, 154 (1935).

[153] U. Fano, "Effects of Configuration Interaction on Intensities and Phase Shifts," Physical Review 124, 1866 (1961).

[154] A. Johnson, C. Marcus, M. Hanson, and A. Gossard, "Coulomb-Modified Fano Resonance in a One-Lead Quantum Dot," Physical Review Letters 93, 106803 (2004).

[155] K. Kobayashi, H. Aikawa, A. Sano, S. Katsumoto, and Y. Iye, "Fano resonance in a quantum wire with a side-coupled quantum dot," Physical Review B 70, 035319 (2004).

[156] B. Luk'yanchuk, N. I. Zheludev, S. A. Maier, N. J. Halas, P. Nordlander, H. Giessen, and C. T. Chong, "The Fano resonance in plasmonic nanostructures and metamaterials." Nature materials 9, 707 (2010).

[157] A. Miroshnichenko, S. Flach, and Y. Kivshar, "Fano resonances in nanoscale structures," Reviews of Modern Physics 82, 2257 (2010).

[158] L. Rayleigh, "On the Dynamical Theory of Gratings," Proceedings of the Royal Society A: Mathematical, Physical and Engineering Sciences 79, 399 (1907).

[159] M. Sarrazin, J.-P. Vigneron, and J.-M. Vigoureux, "Role of Wood anomalies in optical properties of thin metallic films with a bidimensional array of subwavelength holes," Physical Review B 67, 085415 (2003).

[160] Y. S. Joe, A. M. Satanin, and C. S. Kim, “Classical analogy of Fano resonances," Physica Scripta 74, 259 (2006).

[161] N. Verellen, Y. Sonnefraud, H. Sobhani, F. Hao, V. V. Moshchalkov, P. Van Dorpe, P. Nordlander, and S. A. Maier, "Fano resonances in individual coherent plasmonic nanocavities." Nano letters 9, 1663 (2009).

[162] F. Hao, Y. Sonnefraud, P. Van Dorpe, S. A. Maier, N. J. Halas, and P. Nordlander, "Symmetry breaking in plasmonic nanocavities: subradiant LSPR sensing and a tunable Fano resonance." Nano letters 8, 3983 (2008).

[163] M. Klein, T. Tritschler, M. Wegener, and S. Linden, "Lineshape of harmonic generation by metallic nanoparticles and metallic photonic crystal slabs," Physical Review B 72, 115113 (2005). 
[164] V. Fedotov, M. Rose, S. Prosvirnin, N. Papasimakis, and N. Zheludev, “Sharp Trapped-Mode Resonances in Planar Metamaterials with a Broken Structural Symmetry," Physical Review Letters 99, 147401 (2007).

[165] F. Shafiei, F. Monticone, K. Q. Le, X.-X. Liu, T. Hartsfield, A. Alù, and X. Li, "A subwavelength plasmonic metamolecule exhibiting magnetic-based optical Fano resonance." Nature nanotechnology 8, 95 (2013).

[166] S. E. Harris, "Electromagnetically Induced Transparency," Physics Today 50, 36 (1997).

[167] M. Fleischhauer, A. Imamoglu, and J. Marangos, "Electromagnetically induced transparency: Optics in coherent media," Reviews of Modern Physics 77, 633 (2005).

[168] K.-J. Boller, A. Imamolu, and S. Harris, "Observation of electromagnetically induced transparency," Physical Review Letters 66, 2593 (1991).

[169] Y.-q. Li and M. Xiao, "Observation of quantum interference between dressed states in an electromagnetically induced transparency," Physical Review A 51, 4959 (1995).

[170] L. V. Hau, S. E. Harris, Z. Dutton, and C. H. Behroozi, "Light speed reduction to 17 metres per second in an ultracold atomic gas," Nature 397, 594 (1999).

[171] D. Phillips, A. Fleischhauer, A. Mair, R. Walsworth, and M. Lukin, "Storage of Light in Atomic Vapor," Physical Review Letters 86, 783 (2001).

[172] J. J. Longdell, E. Fraval, M. J. Sellars, and N. B. Manson, "Stopped Light with Storage Times Greater than One Second Using Electromagnetically Induced Transparency in a Solid," Physical Review Letters 95, 063601 (2005).

[173] G. Shvets and J. Wurtele, "Transparency of Magnetized Plasma at the Cyclotron Frequency," Physical Review Letters 89, 115003 (2002).

[174] C. L. Garrido Alzar, M. A. G. Martinez, and P. Nussenzveig, "Classical analog of electromagnetically induced transparency," American Journal of Physics 70, 37 (2002).

[175] A. Litvak and M. Tokman, "Electromagnetically Induced Transparency in Ensembles of Classical Oscillators," Physical Review Letters 88, 095003 (2002).

[176] L. Maleki, A. B. Matsko, A. A. Savchenkov, and V. S. Ilchenko, “Tunable delay line with interacting whispering-gallery-mode resonators," Optics Letters 29, 626 (2004).

[177] D. Smith, H. Chang, K. Fuller, A. Rosenberger, and R. Boyd, "Coupled-resonatorinduced transparency," Physical Review A 69, 063804 (2004). 
[178] Q. Xu, S. Sandhu, M. Povinelli, J. Shakya, S. Fan, and M. Lipson, “Experimental Realization of an On-Chip All-Optical Analogue to Electromagnetically Induced Transparency," Physical Review Letters 96, 123901 (2006).

[179] K. Totsuka, N. Kobayashi, and M. Tomita, "Slow Light in Coupled-ResonatorInduced Transparency," Physical Review Letters 98, 213904 (2007).

[180] J. B. Khurgin, "Optical buffers based on slow light in electromagnetically induced transparent media and coupled resonator structures: comparative analysis," Journal of the Optical Society of America B 22, 1062 (2005).

[181] A. Yariv, Y. Xu, R. K. Lee, and A. Scherer, “Coupled-resonator optical waveguide: a proposal and analysis," Optics Letters 24, 711 (1999).

[182] S. Zhang, D. A. Genov, Y. Wang, M. Liu, and X. Zhang, "Plasmon-Induced Transparency in Metamaterials," Physical Review Letters 101, 047401 (2008).

[183] N. Liu, L. Langguth, T. Weiss, J. Kästel, M. Fleischhauer, T. Pfau, and H. Giessen, "Plasmonic analogue of electromagnetically induced transparency at the Drude damping limit." Nature materials 8, 758 (2009).

[184] Y. Lu, H. Xu, J. Y. Rhee, W. H. Jang, B. S. Ham, and Y. Lee, "Magnetic plasmon resonance: Underlying route to plasmonic electromagnetically induced transparency in metamaterials," Physical Review B 82, 195112 (2010).

[185] G. Lenz, B. Eggleton, C. Madsen, and R. Slusher, "Optical delay lines based on optical filters," IEEE Journal of Quantum Electronics 37, 525 (2001).

[186] M. Yanik and S. Fan, "Stopping Light All Optically," Physical Review Letters 92, 083901 (2004).

[187] M. Yanik, W. Suh, Z. Wang, and S. Fan, "Stopping Light in a Waveguide with an All-Optical Analog of Electromagnetically Induced Transparency," Physical Review Letters 93, 233903 (2004).

[188] M. Yanik and S. Fan, "Stopping and storing light coherently," Physical Review A 71, 013803 (2005).

[189] M. Yanik and S. Fan, "Time Reversal of Light with Linear Optics and Modulators," Physical Review Letters 93, 173903 (2004).

[190] M. F. Yanik and S. Fan, "Dynamic Photonic Structures: Stopping, Storage, and Time Reversal of Light," Studies in Applied Mathematics 115, 233 (2005).

[191] S. F. Preble, Q. Xu, and M. Lipson, "Changing the colour of light in a silicon resonator," Nature Photonics 1, 293 (2007). 
[192] J. B. Khurgin, "Dispersion and loss limitations on the performance of optical delay lines based on coupled resonant structures," Optics Letters 32, 133 (2007).

[193] Y. Xu, Y. Li, R. Lee, and A. Yariv, "Scattering-theory analysis of waveguideresonator coupling," Physical Review E 62, 7389 (2000).

[194] Z. S. Yang, N. H. Kwong, R. Binder, and A. L. Smirl, “Distortionless light pulse delay in quantum-well Bragg structures." Optics letters 30, 2790 (2005).

[195] Z. S. Yang, N. H. Kwong, R. Binder, and A. L. Smirl, "Stopping, storing, and releasing light in quantum-well Bragg structures," Journal of the Optical Society of America B 22, 2144 (2005).

[196] J. P. Prineas, W. J. Johnston, M. Yildirim, J. Zhao, and A. L. Smirl, “Tunable slow light in Bragg-spaced quantum wells," Applied Physics Letters 89, 241106 (2006).

[197] R. Kekatpure, E. Barnard, W. Cai, and M. Brongersma, "Phase-Coupled Plasmon-Induced Transparency," Physical Review Letters 104, 243902 (2010).

[198] R. Marqués, J. Martel, F. Mesa, and F. Medina, "Left-Handed-Media Simulation and Transmission of EM Waves in Subwavelength Split-Ring-Resonator-Loaded Metallic Waveguides," Physical Review Letters 89, 183901 (2002).

[199] M. Navarro-Cía, F. Falcone, M. Beruete, I. Arnedo, J. Illescas, A. Marcotegui, M. Laso, and T. Lopetegi, "Left-handed behavior in a microstrip line loaded with squared split-ring resonators and an EBG pattern," Microwave and Optical Technology Letters 49, 2689 (2007).

[200] R. R. Syms, E. Shamonina, and L. Solymar, "Positive and negative refraction of magnetoinductive waves in two dimensions," The European Physical Journal B 46, 301 (2005).

[201] N. Moiseyev, Non-Hermitian Quantum Mechanics (Cambridge University Press, 2011).

[202] C. M. Bender, "Making sense of non-Hermitian Hamiltonians," Reports on Progress in Physics 70, 947 (2007).

[203] I. Gilary, P. Kaprálová-Žd'ánská, and N. Moiseyev, “Ab initio calculation of harmonic generation spectra of helium using a time-dependent non-Hermitian formalism," Physical Review A 74, 052505 (2006).

[204] P. Ginzburg, A. Hayat, V. Vishnyakov, and M. Orenstein, "Photonic logic by linear unidirectional interference," Optics Express 17, 4251 (2009).

[205] W. Hanle, “Über magnetische Beeinflussung der Polarisation der Resonanzfluoreszenz," Zeitschrift für Physik A Hadrons and Nuclei 30, 93 (1924). 
[206] F. Colegrove, P. Franken, R. Lewis, and R. Sands, "Novel Method of Spectroscopy With Applications to Precision Fine Structure Measurements," Physical Review Letters 3, 420 (1959).

[207] J. O. Stenflo, "The Hanle effect and the diagnostics of turbulent magnetic fields in the solar atmosphere," Solar Physics 80, 209 (1982).

[208] R. Ignace, K. H. Nordsieck, and J. P. Cassinelli, “The Hanle Effect as a Diagnostic of Magnetic Fields in Stellar Envelopes. I. Theoretical Results for Integrated Line Profiles," The Astrophysical Journal 486, 550 (1997).

[209] E. H. Brandt, “Levitation in physics." Science 243, 349 (1989).

[210] M. Tinkham, Introduction to Superconductivity: Second Edition (Dover Books on Physics) (Vol i) (Dover Publications, 2004).

[211] F. London, Superfluids Volume 1: Macroscopic Theory of Superconductivity. (Dover Pub., 1961).

[212] W. Spitzer, D. Kleinman, and D. Walsh, "Infrared Properties of Hexagonal Silicon Carbide," Physical Review 113, 127 (1959).

[213] M. Silveirinha and N. Engheta, "Tunneling of Electromagnetic Energy through Subwavelength Channels and Bends using $\epsilon$-Near-Zero Materials," Physical Review Letters 97, 157403 (2006).

[214] A. Alù, M. Silveirinha, A. Salandrino, and N. Engheta, "Epsilon-near-zero metamaterials and electromagnetic sources: Tailoring the radiation phase pattern," Physical Review B 75, 155410 (2007).

[215] N. Engheta, "Circuits with light at nanoscales: optical nanocircuits inspired by metamaterials." Science 317, 1698 (2007).

[216] F. Rosa, D. Dalvit, and P. Milonni, "Casimir-Lifshitz Theory and Metamaterials," Physical Review Letters 100, 183602 (2008).

[217] R. Zhao, J. Zhou, T. Koschny, E. Economou, and C. Soukoulis, "Repulsive Casimir Force in Chiral Metamaterials," Physical Review Letters 103, 103602 (2009).

[218] M. Silveirinha, "Casimir interaction between metal-dielectric metamaterial slabs: Attraction at all macroscopic distances," Physical Review B 82, 085101 (2010).

[219] S. I. Maslovski and M. G. Silveirinha, "Mimicking Boyer's Casimir repulsion with a nanowire material," Physical Review A 83, 022508 (2011). 
[220] J. R. Arias-González and M. Nieto-Vesperinas, "Optical forces on small particles: attractive and repulsive nature and plasmon-resonance conditions," Journal of the Optical Society of America A 20, 1201 (2003).

[221] K.-S. Lee and M. a. El-Sayed, "Gold and silver nanoparticles in sensing and imaging: sensitivity of plasmon response to size, shape, and metal composition." The journal of physical chemistry. B 110, 19220 (2006).

[222] K. L. Kelly, E. Coronado, L. L. Zhao, and G. C. Schatz, "The Optical Properties of Metal Nanoparticles: The Influence of Size, Shape, and Dielectric Environment," The Journal of Physical Chemistry B 107, 668 (2003).

[223] L. S. Jung, C. T. Campbell, T. M. Chinowsky, M. N. Mar, and S. S. Yee, "Quantitative Interpretation of the Response of Surface Plasmon Resonance Sensors to Adsorbed Films," Langmuir 14, 5636 (1998).

[224] A. J. Haes, W. P. Hall, L. Chang, W. L. Klein, and R. P. Van Duyne, "A Localized Surface Plasmon Resonance Biosensor: First Steps toward an Assay for Alzheimer's Disease," Nano Letters 4, 1029 (2004).

[225] E. M. Larsson, J. Alegret, M. Käll, and D. S. Sutherland, “Sensing characteristics of NIR localized surface plasmon resonances in gold nanorings for application as ultrasensitive biosensors." Nano letters 7, 1256 (2007).

[226] W. Kubo and S. Fujikawa, "Au double nanopillars with nanogap for plasmonic sensor." Nano letters 11, 8 (2011).

[227] N. Verellen, P. Van Dorpe, C. Huang, K. Lodewijks, G. A. E. Vandenbosch, L. Lagae, and V. V. Moshchalkov, "Plasmon line shaping using nanocrosses for high sensitivity localized surface plasmon resonance sensing." Nano letters 11, 391 (2011).

[228] Y. Gu, Q. Li, J. Xiao, K. Wu, and G. P. Wang, "Plasmonic metamaterials for ultrasensitive refractive index sensing at near infrared," Journal of Applied Physics 109, 023104 (2011).

[229] J. Ye and P. Van Dorpe, "Improvement of Figure of Merit for Gold Nanobar Array Plasmonic Sensors," Plasmonics 6, 665 (2011).

[230] J. B. Lassiter, H. Sobhani, J. A. Fan, J. Kundu, F. Capasso, P. Nordlander, and N. J. Halas, "Fano resonances in plasmonic nanoclusters: geometrical and chemical tunability." Nano letters 10, 3184 (2010).

[231] Y. Chu, E. Schonbrun, T. Yang, and K. B. Crozier, "Experimental observation of narrow surface plasmon resonances in gold nanoparticle arrays," Applied Physics Letters 93, 181108 (2008). 
[232] Y.-C. Chang, H.-C. Chung, S.-C. Lu, and T.-F. Guo, “A large-scale sub-100 nm Au nanodisk array fabricated using nanospherical-lens lithography: a low-cost localized surface plasmon resonance sensor." Nanotechnology 24, 095302 (2013).

[233] A. V. Kabashin, P. Evans, S. Pastkovsky, W. Hendren, G. A. Wurtz, R. Atkinson, R. Pollard, V. A. Podolskiy, and A. V. Zayats, "Plasmonic nanorod metamaterials for biosensing." Nature materials 8, 867 (2009).

[234] A. D. McFarland and R. P. Van Duyne, "Single Silver Nanoparticles as Real-Time Optical Sensors with Zeptomole Sensitivity," Nano Letters 3, 1057 (2003).

[235] M. Février, P. Gogol, G. Barbillon, A. Aassime, R. Mégy, B. Bartenlian, J.-m. Lourtioz, and B. Dagens, "Integration of short gold nanoparticles chain on SOI waveguide toward compact integrated bio-sensors," Optics Express 20, 17402 (2012).

[236] M. Février, P. Gogol, A. Aassime, R. Mégy, C. Delacour, A. Chelnokov, A. Apuzzo, S. Blaize, J.-M. Lourtioz, and B. Dagens, "Giant coupling effect between metal nanoparticle chain and optical waveguide." Nano letters 12, 1032 (2012).

[237] A. Apuzzo, M. Fevrier, R. Salas-Montiel, A. Bruyant, A. Chelnokov, G. Lerondel, B. Dagens, and S. Blaize, "Observation of near-field dipolar interactions involved in metal nanoparticles chain waveguide." Nano letters (2013), $10.1021 / \mathrm{nl} 304164 \mathrm{y}$.

[238] Z. Liu, J. M. Steele, W. Srituravanich, Y. Pikus, C. Sun, and X. Zhang, "Focusing surface plasmons with a plasmonic lens." Nano letters 5, 1726 (2005).

[239] F. Zhou, Y. Liu, and W. Cai, "Plasmonic holographic imaging with V-shaped nanoantenna array," Optics Express 21, 4348 (2013).

[240] M. Aeschlimann, M. Bauer, D. Bayer, T. Brixner, S. Cunovic, A. Fischer, P. Melchior, W. Pfeiffer, M. Rohmer, C. Schneider, C. Strüber, P. Tuchscherer, and D. V. Voronine, "Optimal open-loop near-field control of plasmonic nanostructures," New Journal of Physics 14, 033030 (2012).

[241] Z. Liu, Y. Wang, J. Yao, H. Lee, W. Srituravanich, and X. Zhang, "Broad band two-dimensional manipulation of surface plasmons." Nano letters 9, 462 (2009).

[242] R. Merlin, "Radiationless electromagnetic interference: evanescent-field lenses and perfect focusing." Science 317, 927 (2007).

[243] B. Zeng, X. Yang, C. Wang, and X. Luo, "Plasmonic interference nanolithography with a double-layer planar silver lens structure," Optics Express 17, 16783 (2009).

[244] N. Yu, P. Genevet, M. A. Kats, F. Aieta, J.-P. Tetienne, F. Capasso, and Z. Gaburro, "Light propagation with phase discontinuities: generalized laws of reflection and refraction." Science 334, 333 (2011). 
[245] X. Ni, N. K. Emani, A. V. Kildishev, A. Boltasseva, and V. M. Shalaev, “Broadband light bending with plasmonic nanoantennas." Science 335, 427 (2012).

[246] P. Genevet, N. Yu, F. Aieta, J. Lin, M. A. Kats, R. Blanchard, M. O. Scully, Z. Gaburro, and F. Capasso, "Ultra-thin plasmonic optical vortex plate based on phase discontinuities," Applied Physics Letters 100, 013101 (2012).

[247] F. Aieta, P. Genevet, N. Yu, M. A. Kats, Z. Gaburro, and F. Capasso, “Out-of-plane reflection and refraction of light by anisotropic optical antenna metasurfaces with phase discontinuities." Nano letters 12, 1702 (2012).

[248] D. Pozar, S. Targonski, and R. Pokuls, "A shaped-beam microstrip patch reflectarray," IEEE Transactions on Antennas and Propagation 47, 1167 (1999).

[249] A. Alù and N. Engheta, "Achieving transparency with plasmonic and metamaterial coatings," Physical Review E 72, 016623 (2005).

[250] A. Alù and N. Engheta, "Cloaking a receiving antenna or a sensor with plasmonic metamaterials," Metamaterials 4, 153 (2010).

[251] A. Alù and N. Engheta, "Multifrequency Optical Invisibility Cloak with Layered Plasmonic Shells," Physical Review Letters 100, 113901 (2008).

[252] L. Soldano and E. Pennings, "Optical multi-mode interference devices based on self-imaging: principles and applications," Journal of Lightwave Technology 13, 615 (1995).

[253] B. Steinberger, A. Hohenau, H. Ditlbacher, A. L. Stepanov, A. Drezet, F. R. Aussenegg, A. Leitner, and J. R. Krenn, "Dielectric stripes on gold as surface plasmon waveguides," Applied Physics Letters 88, 094104 (2006).

[254] Z. Han and S. He, "Multimode interference effect in plasmonic subwavelength waveguides and an ultra-compact power splitter," Optics Communications 278, 199 (2007).

[255] Y. Kou and X. Chen, "Multimode interference demultiplexers and splitters in metal-insulator-metal waveguides," Optics Express 19, 6042 (2011).

[256] Y.-J. Tsai, A. Degiron, N. M. Jokerst, and D. R. Smith, "Plasmonic multi-mode interference couplers," Optics Express 17, 17471 (2009).

[257] J. a. Dionne, K. Diest, L. a. Sweatlock, and H. a. Atwater, "PlasMOStor: a metal-oxide-Si field effect plasmonic modulator." Nano letters 9, 897 (2009).

[258] P. Berini, "Figures of merit for surface plasmon waveguides," Optics Express 14, 13030 (2006). 
[259] R. Buckley and P. Berini, "Figures of merit for 2D surface plasmon waveguides and application to metal stripes," Optics Express 15, 12174 (2007).

[260] L. Chen, J. Shakya, and M. Lipson, "Subwavelength confinement in an integrated metal slot waveguide on silicon," Optics Letters 31, 2133 (2006).

[261] D. Dai and S. He, "Low-loss hybrid plasmonic waveguide with double low-index nano-slots," Optics Express 18, 17958 (2010).

[262] J. T. Kim, J. J. Ju, S. Park, M.-s. Kim, S. K. Park, and S.-Y. Shin, “Hybrid plasmonic waveguide for low-loss lightwave guiding," Optics Express 18, 2808 (2010).

[263] S. A. Maier, P. E. Barclay, T. J. Johnson, M. D. Friedman, and O. Painter, “Lowloss fiber accessible plasmon waveguide for planar energy guiding and sensing," Applied Physics Letters 84, 3990 (2004).

[264] R. F. Oulton, V. J. Sorger, D. A. Genov, D. F. P. Pile, and X. Zhang, "A hybrid plasmonic waveguide for subwavelength confinement and long-range propagation," Nature Photonics 2, 496 (2008).

[265] D. a. Bobb, G. Zhu, M. Mayy, a. V. Gavrilenko, P. Mead, V. I. Gavrilenko, and M. a. Noginov, "Engineering of low-loss metal for nanoplasmonic and metamaterials applications," Applied Physics Letters 95, 151102 (2009).

[266] D. Bergman and M. Stockman, "Surface Plasmon Amplification by Stimulated Emission of Radiation: Quantum Generation of Coherent Surface Plasmons in Nanosystems," Physical Review Letters 90, 027402 (2003).

[267] S. Xiao, V. P. Drachev, A. V. Kildishev, X. Ni, U. K. Chettiar, H.-K. Yuan, and V. M. Shalaev, "Loss-free and active optical negative-index metamaterials." Nature 466, 735 (2010).

[268] J. Y. Lee, B. H. Hong, W. Y. Kim, S. K. Min, Y. Kim, M. V. Jouravlev, R. Bose, K. S. Kim, I.-C. Hwang, L. J. Kaufman, C. W. Wong, P. Kim, and K. S. Kim, "Near-field focusing and magnification through self-assembled nanoscale spherical lenses," Nature 460, 498 (2009).

[269] G. Eleftheriades and A. Wong, "Holography-Inspired Screens for SubWavelength Focusing in the Near Field," IEEE Microwave and Wireless Components Letters 18, 236 (2008).

[270] L. Helseth, "The almost perfect lens and focusing of evanescent waves," Optics Communications 281, 1981 (2008).

[271] M. Stockman, S. Faleev, and D. Bergman, "Coherent Control of Femtosecond Energy Localization in Nanosystems," Physical Review Letters 88, 067402 (2002). 
[272] M. Sukharev and T. Seideman, "Phase and polarization control as a route to plasmonic nanodevices." Nano letters 6, 715 (2006).

[273] H. Kim and B. Lee, "Unidirectional Surface Plasmon Polariton Excitation on Single Slit with Oblique Backside Illumination," Plasmonics 4, 153 (2009).

[274] N. Bonod, E. Popov, L. Li, and B. Chernov, "Unidirectional excitation of surface plasmons by slanted gratings," Optics Express 15, 11427 (2007).

[275] J.-S. Bouillard, S. Vilain, W. Dickson, G. A. Wurtz, and A. V. Zayats, "Broadband and broadangle SPP antennas based on plasmonic crystals with linear chirp." Scientific reports 2, 829 (2012).

[276] X. Li, Q. Tan, B. Bai, and G. Jin, "Experimental demonstration of tunable directional excitation of surface plasmon polaritons with a subwavelength metallic double slit," Applied Physics Letters 98, 251109 (2011).

[277] I. P. Radko, S. I. Bozhevolnyi, G. Brucoli, L. Martin-Moreno, F. J. Garcia-Vidal, and A. Boltasseva, "Efficient unidirectional ridge excitation of surface plasmons," Optics Express 17, 7228 (2009).

[278] Y. Liu, S. Palomba, Y. Park, T. Zentgraf, X. Yin, and X. Zhang, "Compact magnetic antennas for directional excitation of surface plasmons." Nano letters 12, 4853 (2012).

[279] F. Hellman, E. M. Gyorgy, D. W. Johnson, H. M. O'Bryan, and R. C. Sherwood, "Levitation of a magnet over a flat type II superconductor," Journal of Applied Physics 63, 447 (1988). 


\section{Author's merits}

\section{Journal Papers}

[PAPER A] F. J. Rodríguez-Fortuño, C. García-Meca, R. Ortuño, J. Martí, and A. Martínez, "Modeling high-order plasmon resonances of a U-shaped nanowire used to build a negative-index metamaterial," Physical Review B 79, 075103 (2009).

[PAPER B] F. J. Rodríguez-Fortuño, R. Ortuño, C. García-Meca, J. Martí, and A. Martínez, "High order standing-wave plasmon resonances in silver u-shaped nanowires," Journal of Applied Physics 112, 103104 (2012).

[PAPER C] F. J. Rodríguez-Fortuño, C. García-Meca, R. Ortuño, J. Martí, and A. Martínez, "Coaxial plasmonic waveguide array as a negative-index metamaterial," Optics Letters 34, 3325 (2009).

[PAPER D] F. J. Rodríguez-Fortuño, B. Tomás-Navarro, C. García-Meca, R. Ortuño, J. Martí, and A. Martínez, "Zero-bandwidth mode in a split-ring-resonatorloaded one-dimensional photonic crystal," Physical Review B 81, 233101 (2010).

[PAPER E] P. Ginzburg*, F. J. Rodríguez-Fortuño*, A. Martínez, and A. V. Zayats, "Analogue of the quantum Hanle effect and polarization conversion in non-Hermitian plasmonic metamaterials." Nano Letters 12, 6309 (2012) ${ }^{*}$ These authors contributed equally.

[PAPER F] F. J. Rodríguez-Fortuño, M. Martínez-Marco, B. Tomás-Navarro, R. Ortuño, J. Martí, A. Martínez, and P. J. Rodríguez-Cantó, “Highlysensitive chemical detection in the infrared regime using plasmonic gold nanocrosses," Applied Physics Letters 98, 133118 (2011).

[PAPER G] F. J. Rodríguez-Fortuño and N. Engheta, "Avoiding metallic walls: Use of modal superposition in plasmonic waveguides to reduce propagation loss," Physical Review B 85, 115421 (2012). 
[PAPER H] F. J. Rodríguez-Fortuño, G. Marino, P. Ginzburg, D. O'Connor, A. Martínez, G. A. Wurtz, and A. V. Zayats, "Near field interference for the unidirectional excitation of electromagnetic guided modes," Science 340,328 (2013).

[Paper 9] C. García-Meca, R. Ortuño, F. J. Rodríguez-Fortuño, J. Martí, and A. Martínez, "Double-negative polarization-independent fishnet metamaterial in the visible spectrum," Optics Letters 34, 1603 (2009).

[Paper 10] C. García-Meca, R. Ortuño, F. J. Rodríguez-Fortuño, J. Martí, and A. Martínez, "Negative refractive index metamaterials aided by extraordinary optical transmission," Optics Express 17, 6026 (2009).

[Paper 11] A. Martínez, C. García-Meca, R. Ortuño, F. J. Rodríguez-Fortuño, and J. Martí, "Metamaterials for optical security," Applied Physics Letters 94, 251106 (2009).

[Paper 12] R. Ortuño, C. García-Meca, F. J. Rodríguez-Fortuño, A. Hakansson, A. Griol, J. Hurtado, J. A. Ayucar, L. Bellieres, P. J. Rodríguez, F. LópezRoyo, J. Martí, and A. Martínez, "Midinfrared filters based on extraordinary optical transmission through subwavelength structured gold films," Journal of Applied Physics 106, 124313 (2009).

[Paper 13] R. Ortuño, C. García-Meca, F. J. Rodríguez-Fortuño, J. Martí, and A. Martínez, "Role of surface plasmon polaritons on optical transmission through double layer metallic hole arrays," Physical Review B 79, 075425 (2009).

[Paper 14] R. Ortuño, C. García-Meca, F. J. Rodríguez-Fortuño, J. Martí, and A. Martínez, "Multiple extraordinary optical transmission peaks from evanescent coupling in perforated metal plates surrounded by dielectrics," Optics Express 18, 7893 (2010).

[Paper 15] R. Ortuño, C. García-Meca, F. J. Rodríguez-Fortuño, and A. Martínez, "Enlarging the negative-index bandwidth of optical metamaterials by hybridized plasmon resonances," Optics Letters 35, 4205 (2010).

[Paper 16] P. Rodríguez-Cantó, M. Martínez-Marco, F. J. Rodríguez-Fortuño, B. Tomás-Navarro, R. Ortuño, S. Peransí-Llopis, and A. Martínez, "Demonstration of near infrared gas sensing using gold nanodisks on functionalized silicon," Optics Express 19, 7664 (2011).

[Paper 17] C. García-Meca, M. M. Tung, J. V. Galán, R. Ortuño, F. J. RodríguezFortuño, J. Martí, and A. Martínez, "Squeezing and expanding light without reflections via transformation optics," Optics Express 19, 3562 (2011). 
[Paper 18] P. Ginzburg, F. J. Rodríguez-Fortuño, G. A. Wurtz, W. Dickson, A. Murphy, F. Morgan, R. J. Pollard, I. Iorsh, A. Atrashchenko, P. A. Belov, Y. S. Kivshar, A. Nevet, G. Ankonina, M. Orenstein, and A. V. Zayats, "Manipulating polarization of light with ultrathin epsilon-near-zero metamaterials," Optics Express 21, 14907 (2013).

\section{Pre-print submissions}

[Paper 19] F. J. Rodríguez-Fortuño, A. Vakil, and N. Engheta, "Electric Levitation Using Epsilon-Near-Zero Metamaterials," ArXiv , 18 (2013), arXiv:1302.5827

[Paper 20] F. J. Rodríguez-Fortuño, D. Puerto, A. Griol, L. Bellieres, J. Martí, and A. Martínez, "Sorting polarized photons with a single scatterer," ArXiv , 9 (2013), arXiv:1306.4205

\section{Conferences}

[Conf 1] C. Garcia-Meca, M. Rubén Ortuño, F. J. Rodríguez-Fortuño, A. J. Martínez Abiétar, and J. Martí, "Extraordinary optical transmission with negative index of refraction," in 2nd International Congress on Advanced Electromagnetic Materials in Microwaves and Optics, (Pamplona), 2008.

[Conf 2] M. Rubén Ortuño, C. Garcia-Meca, F. J. Rodríguez-Fortuño, A. J. Martínez Abiétar, and J. Martí, "Effect of internal and external surface plasmons in the enhanced transmisión through double-layer metallic hole arrays," in 2nd International Congress on Advanced Electromagnetic Materials in Microwaves and Optics, (Pamplona), 2008.

[Conf 3] F. J. Rodríguez-Fortuño, C. Garcia-Meca, R. Ortuno, J. Martí, and A. Martínez, "Negative index metamaterial through high-order plasmon resonances on u-shaped nanowires," in 2009 IEEE/LEOS Winter Topicals Meeting Series, (Innsbruck), 2009.

[Conf 4] C. García-Meca, R. Ortuño, F. J. Rodríguez-Fortuño, J. Marti, and A. Martinez, "Double-negative polarization-independent fishnet metamaterial operating in the visible spectrum," in 2009 IEEE/LEOS Winter Topicals Meeting Series, (Innsbruck), 2009.

[Conf 5] F. J. Rodríguez-Fortuño, R. Ortuño, C. García-Meca, J. Martí, and A. Martínez, "Modeling and understanding plasmonic resonances in optical metamaterials," in 3rd Young Scientist Meeting on Metamaterials (YSMM'09), (Madrid), 2009. 
[Conf 6] F. J. Rodríguez-Fortuño, R. Ortuño, C. García-Meca, M. Navarro-Cía, F. Falcone, J. Martí, and A. Martínez, "Split-ring resonators achieve transmission through a photonic crystal bandgap," in 3rd International Congress on Advanced Electromagnetic Materials in Microwaves and Optics, (London), 2009.

[Conf 7] C. García-Meca, R. Ortuño, F. J. Rodríguez-Fortuño, J. Martí, and A. Martínez, "Strong magnetism at visible wavelengths via coupled silver nanohoops," in 3rd International Congress on Advanced Electromagnetic Materials in Microwaves and Optics, (London), 2009.

[Conf 8] R. Ortuño, C. García-Meca, F. J. Rodríguez-Fortuño, J. Martí, and A. Martínez, "MIR Filters based on extraordinary optical transmission through Nanostructures Gold Films," in 3rd International Congress on Advanced Electromagnetic Materials in Microwaves and Optics, (London), 2009.

[Conf 9] R. Ortuño, C. García-Meca, F. J. Rodríguez-Fortuño, J. Martí, and A. Martínez, "Multiple Extraordinary Optical Transmission Peaks from Evanescent Coupling in Perforated Metal Plates Surrounded by Dielectric," in 3rd International Congress on Advanced Electromagnetic Materials in Microwaves and Optics, (London), 2009.

[Conf 10] C. García-Meca, M. Tung, J. V. Galán, R. Ortuño, F. J. Rodríguez-Fortuño, J. Martí, and A. Martínez, "Light compression without reflections," in SPIE Photonics Europe, (Brussels), p. 771121, 2010.

[Conf 11] R. Ortuño, C. García-Meca, F. J. Rodríguez-Fortuño, J. Martí, and A. Martínez, "Enlarged negative effective index bandwidth from fishnet metamaterials," in SPIE Photonics Europe, (Brussels), p. 771108, 2010.

[Conf 12] B. Tomás-Navarro, F. J. Rodríguez-Fortuño, R. Ortuño, C. García-Meca, and A. Martínez, "Zero bandwidth mode on a split ring resonator loaded waveguide at cutoff," in Conferencia Española de Nanofotónica, (Segovia), 2010.

[Conf 13] M. Martínez-Marco, F. J. Rodríguez-Fortuño, P. Rodríguez-Cantó, B. n. Tomás-Navarro, R. Ortuño, and A. Martínez, "Plasmonic sensor based on gold nanodisk structures for toxic organic molecule detection," in Conferencia Española de Nanofotónica, (Segovia), 2010.

[Conf 14] M. Martínez-Marco, F. J. Rodríguez-Fortuño, P. Rodríguez-Cantó, B. Tomás-Navarro, R. Ortuño, and A. Martínez, “Plasmonic sensor based on gold nanodisk structures for toxic organic molecules detection," in 4th International Congress on Advanced Electromagnetic Materials in Microwaves and Optics, (Karlsruhe), 2010. 
[Conf 15] F. J. Rodríguez-Fortuño, B. Tomás-Navarro, C. García-Meca, R. Ortuño, and A. Martínez, "Split-ring-resonators inserted in a photonic crystal achieve a zerobandwidth passband," in 4th International Congress on Advanced Electromagnetic Materials in Microwaves and Optics, (Karlsruhe), 2010.

[Conf 16] M. Lorente-Crespo, F. J. Rodríguez-Fortuño, R. Ortuño, C. García-Meca, and A. Martínez, "Magnetic metamaterials in the visible range based on nano-hoops and nano-squares," in 4th Young Scientist Meeting in Metamaterials, (Valencia), 2011.

[Conf 17] F. J. Rodríguez-Fortuño, M. Martínez-Marco, B. Tomás-Navarro, R. Ortuño, A. Martínez, and P. Rodríguez-Cantó, "Chemical sensor using the localized surface plasmon resonance of arrayed gold nanocrosses," in 4th Young Scientist Meeting in Metamaterials, (Valencia), 2011.

[Conf 18] R. Ortuño, C. García-Meca, F. J. Rodríguez-Fortuño, and A. Martínez, "Extraordinary Transmission and Light Confinement in Subwavelength Metallic Films Apertures," in 29th Progress in Electromagnetics Research Symposium, (Marrakesh), 2011.

[Conf 19] C. García-Meca, R. Ortuño, F. J. Rodríguez-Fortuño, J. Martí, and A. Martínez, "Scaling slabs based on transformation optics for immersion lenses and angular filters," in 5th International Congress on Advanced Electromagnetic Materials in Microwaves and Optics, (Barcelona), 2011.

[Conf 20] R. Ortuño, C. García-Meca, F. J. Rodríguez-Fortuño, J. Martí, and A. Martínez, "Extraordinary Transmission through Metamaterials Arrays at THz Frequencies," in 4th Young Scientist Meeting in Metamaterials, (Valencia), 2011.

[Conf 21] F. J. Rodríguez-Fortuño and N. Engheta, "Wall-Avoiding Field Distributions in Plasmonic Waveguides," in Frontiers in Optics 2011 / Laser Science XXVII, (San Jose), 2011.

[Conf 22] C. García-Meca, R. Ortuño, F. J. Rodríguez-Fortuño, J. Martí, and A. Martínez, "Flat and reflectionless solid immersion lens based on transformation optics," in 4th Young Scientist Meeting in Metamaterials, (Valencia), 2011.

[Conf 23] I. Alepuz-Benaches, C. García-Meca, F. J. Rodríguez-Fortuño, R. Ortuño, M. Lorente-Crespo, A. Griol, and A. Martínez, "Strong magnetic resonance of coupled aluminum nanodisks on top of a silicon waveguide," in SPIE Photonics Europe 2012, (Brussels), 2012. 
[Conf 24] F. J. Rodríguez-Fortuño and N. Engheta, "Three-dimensional stereo filter metamaterial," in 3rd International Conference on Metamaterials, Photonic Crystals and Plasmonics, (Paris), 2012.

[Conf 25] F. J. Rodríguez-Fortuño, P. Ginzburg, A. Martínez, and A. V. Zayats, "Polarization Conversion in Non-Hermitian Metamaterials: A Plasmonic Analogue to the Quantum Hanle Effect," in 4th International Topical Meeting on Nanophotonics and Metamaterials, (Seefeld), 2013. 


\begin{abstract}
Plasmonics is the science that studies the interaction, at nanoscale dimensions, between light and free electrons in metals, giving rise to wave propagation highly confined to their surface. Plasmonics has a large variety of applications in nanotechnology, such as biological and chemical sensing, spectroscopy, nanolithography, ultra wide band communications integrated on chips, light nanoantennas, filtering, and manipulation of optical signals, among many others. One of the most novel applications is the creation of metamaterials: artificial structures designed to control the propagation of light with fascinating applications such as the perfect lens or the invisibility cloak. Plasmonics and metamaterials are in the forefront of current photonics research thanks to the rise of nanotechnology and nanoscience which opens the door to a great variety of new applications.

This thesis, performed at the Valencia Nanophotonics Technology Center in the UPV, in collaboration with the University of Pennsylvania and King's College London, aims at contributing with new ideas, structures and devices to the fields of plasmonics and metamaterials, including their fabrication and experimental measurement whenever possible. The thesis is not limited to a single application or device, but instead does an extensive exploration of the different sub-fields of plasmonics in search of novel phenomena. The results described are the following:

In the field of negative index metamaterials, two new structures are presented: U-shaped nanowires and coaxial plasmonic waveguides. In the field of plasmonic sensing, the design and experimental realization of a high-performance sensor based on metallic nanocrosses is presented. Also detailed are the theoretical descriptions of a novel device for slow-light and time-reversal of light pulses based on metamaterials and photonic crystals, a polarization conversion metamaterial tunable through its losses, a plasmonic analogue of the Meissner effect levitation in superconductors, and a method of loss reduction in plasmonic waveguides through multimode interference. Finally, the theoretical and experimental demonstration of a new fundamental example of near field interference is presented, achieving the unidirectional excitation of photonic modes - whether plasmonic or not - with the near fields of a circularly polarized dipole.
\end{abstract}




\section{Resumen}

La plasmónica es la ciencia que estudia la interacción, a escala nanométrica, entre la luz y los electrones libres de los metales, dando lugar a la propagación de ondas altamente confinadas a su superficie. La plasmónica tiene multitud de aplicaciones en nanotecnología, como son el sensado biológico y químico, espectroscopía, nanolitografía, comunicaciones de banda ultra ancha integradas en chips, nanoantenas para luz, filtrado, y manipulación de señales ópticas, entre muchas otras. Una de las aplicaciones más novedosas es la creación de metamateriales: estructuras artificiales diseñadas para controlar la propagación de la luz, con aplicaciones fascinantes como la lente perfecta o la capa de invisibilidad. La plasmónica y los metamateriales están al frente de la investigación actual en fotónica, gracias al auge de la nanotecnología y la nanociencia, que abre las puertas a una gran cantidad de nuevas aplicaciones.

Esta tesis, desarrollada en el Centro de Tecnología Nanofotónica de Valencia de la UPV, en colaboración con la University of Pennsylvania y King's College London, trata de aportar nuevas ideas, estructuras y dispositivos a los campos de la plasmónica y los metamateriales, tratando de realizar su fabricación y medida experimental cuando sea posible. La tesis no se ciñe a una única aplicación o dispositivo, sino que realiza una extensiva exploración de los diversos sub-campos de la plasmónica en busca de fenómenos novedosos. Los resultados descritos son los siguientes:

En el campo de los metamateriales de índice negativo se presentan dos estructuras: nanocables en forma de $\mathrm{U}$, y guías coaxiales plasmónicas. En el campo del sensado plasmónico se presenta el diseño y la prueba experimental de un sensor de sustancias químicas de altas prestaciones con nanocruces metálicas. También se detallan teóricamente: un novedoso dispositivo para luz lenta e inversión temporal de pulsos basada en metamateriales y cristales fotónicos, un metamaterial para conversión de polarización sintonizable mediante pérdidas, un análogo plasmónico al efecto de levitación Meissner en superconductores y un método de reducción de pérdidas en guías plasmónicas mediante interferencia en guías multimodo. Por último se presenta teórica y experimentalmente un nuevo ejemplo fundamental de interferencia de campo cercano, logrando la excitación unidireccional de modos fotónicos - ya sean plasmónicos o no-mediante los campos cercanos de un dipolo circularmente polarizado. 


\section{Resum}

La plasmònica és la ciència que estudia la interacció, a escala nanométrica, entre la llum i els electrons lliures dels metalls, donant lloc a la propagació d'ones altament confinades a la seua superfície. La plasmònica té multitud d'aplicacions en nanotecnologia, com són el sensado biològic i químic, espectroscòpia, nanolitografía, comunicacions de banda ultra ampla integrades en xips, nanoantenas per a llum, filtrat, i manipulació de senyals òptics, entre moltes altres. Una de les aplicacions més noves és la creació de metamaterials: estructures artificials dissenyades per a controlar la propagació de la llum, amb aplicacions fascinants com la lent perfecta o la capa d'invisibilitat. La plasmónica i els metamaterials estan al capdavant de la investigació actual en fotónica, gràcies a l'auge de la nanotecnologia i la nanociencia, que obri les portes a una gran quantitat de noves aplicacions.

Esta tesi, desenrotllada en el Centre de Tecnologia Nanofotónica de València de la UPV, en col-laboració amb la University of Pennsylvania i King's College London, tracta d'aportar noves idees, estructures i dispositius als camps de la plasmònica i els metamaterials, tractant de realitzar la seua fabricació i mesura experimental quan siga possible. La tesi no se cenyix a una única aplicació o dispositiu, sinó que realitza una extensiva exploració dels diversos subcamps de la plasmònica a la cerca de fenòmens nous. Els resultats descrits són els següents:

En el camp dels metamaterials d'índex negatiu es presenten dos estructures: nanocables en forma de $\mathrm{U}$, i guies coaxials plasmòniques. En el camp del sensado plasmònic es presenta el disseny i la prova experimental d'un sensor de substàncies químiques d'altes prestacions amb nanocruces metàl-liques. També es detallen teòricament: un nou dispositiu per a llum lenta i inversió temporal de polsos basada en metamateriales i vidres fotónicos, un metamaterial per a conversió de polarització sintonizable per mitjà de pèrdues, un anàleg plasmònic a este efecte de levitació Meissner en superconductors i un mètode de reducció de pèrdues en guies plasmòniques per mitjà d'interferència en guies multimodo. Finalment es presenta teòricament i experimentalment un nou exemple fonamental d'interferència de camp pròxim, aconseguint l'excitació unidireccional de modes fotònics - ya siguen plasmònics o noper mitjà dels camps pròxims d'un dipol circularment polaritzat. 